\title{
THE EFFECT OF A SHALLOW LOW VISCOSITY ZONE \\ ON MANTLE CONVECTION AND ITS EXPRESSION \\ AT THE SURFACE OF THE EARTH
}

by

Elizabeth M. Robinson

B.S., Reed College, 1982

SUBMITTED IN PARTIAL FULFILLMENT

OF THE REQUIREMENTS FOR THE DEGREE OF

DOCTOR OF PHILOSOPHY

at the

MASSACHUSETTS INSTITUTE OF TECHNOLOGY

and the

WOODS HOLE OCEANOGRAPHIC INSTITUTION

AUGUST 1987

(C) Elizabeth M. Robinson, 1987

The author hereby grants to MIT and WHOI permission to reproduce and distribute copies of this thesis document in whole or in part.

\section{Signature of Author Joint Progrant in Marine Geology and Geophysics, Massachusetts Institute of Technology and the Woods Hole oceanographic Institution}

Certified by

Barry Parsons, Thesis Supervisor

Accepted by

Chairman of the Joint Committee for Marine Geology and

Geophysics, Massachusetts Institute of Technology and the

Woods Hole Oceanographic Institution 
THE EFFECT OF A SHALLOW LOW VISCOSITY ZONE ON MANTLE FLOW

AND ITS EXPRESSION AT THE SURFACE OF THE EARTH

by

Elizabeth M. Robinson

submitted to the Massachusetts Institute of Technology/

Woods Hole Oceanographic Institution Joint Program in Geology and Geophysics on July 1, 1987, in partial fulfillment of the requirements for the degree of Doctor of Philosophy.

\begin{abstract}
Many features of the oceanic plates cannot be explained by conductive cooling with age. A number of these anomalies require additional convective thermal sources at depths below the plate: mid-plate swells, the evolution of fracture zones, the mean depth and heat flow relationships with age and the observation of small scale $(150-250 \mathrm{~km})$ geoid and topography anomalies in the Central Pacific and Indian oceans. Convective models are presented of the formation and evolution of these features. In particular, the effect of a shallow low viscosity layer in the uppermost mantle on mantle flow and its geoid, topography, gravity and heat flow expression is explored. A simple numerical model is employed of convection in a fluid which has a low viscosity layer lying between a rigid bed and a constant viscosity region. Finite element calculations have been used to determine the effects of (1) the viscosity contrast between the two fluid layers, (2) the thickness of the low viscosity zone, (3) the thickness of the conducting lid, and (4) the Rayleigh number of the fluid based on the viscosity of the lower layer.

A model simple for mid-plate swells is that they are the surface expression of a convection cell driven by a heat flux from below. The low viscosity zone causes the top boundary layer of the convection cell to thin and, at high viscosity contrasts and Rayleigh numbers, it can cause the boundary layer to go unstable. The low viscosity zone also mitigates the transmission of normal stress to the conducting lid so that the topography and geoid anomalies decrease. The geoid anomaly decreases faster than the topography anomaly, however, so that the depth of compensation can appear to be well within the conducting lid. Because the boundary layer is thinned, the elastic plate thickness also decreases and, since the low viscosity allows the fluid to flow faster in the top layer, the uplift time decreases as well. We have compared the results of this modeling to data at the Hawaii, Bermuda, Cape Verde and Marquesas swells, and have found that it can reproduce their
\end{abstract}


observed anomalies. The viscosity contrasts that are required range from $0.2-0.01$, which are in agreement with other estimates of shallow viscosity variation in the upper mantle. Also, the estimated viscosity contrast decreases as the age of the swell increases. This trend is consistent with theoretical estimates of the variation of such a low viscosity zone with age.

Fracture zones juxtapose segments of the oceanic plates of different ages and thermal structures. The flow induced by the horizontal temperature gradient at the fracture zone initially downwells immediately adjacent to the fracture zone on the older side, generating cells on either side of the plume. The time scale and characteristic wavelength of this flow depends initially on the viscosity near the largest temperature gradient in the fluid which, in our model, is the viscosity of the low viscosity layer. They therefore depend on both the Rayleigh number and the viscosity contrast between the layers. Eventually the flow extends throughout the box, and the time scales and the characteristic wavelengths of the flow depend on the thickness and viscosity of both layers. When the Rayleigh number based on the viscosity of the top layer, and the depth of both fluid layers, is less than $10^{6}$, the geoid anomalies of these flows are dominated by the convective signal. When this Rayleigh number exceeds $10^{6}$, the geoid anomalies retain a step across the fracture zone out to large ages. We have compared our results to geoid anomalies over the Udintsev fracture zone, and have found that the predicted geoid anomalies, with high effective Rayleigh numbers, agree at longer wavelengths with the observed anomalies and can produce the observed geoid slope-age behaviour. We have also compared the calculated topographic steps to those predicted by the average depth-age relationships observed in the oceans. We have found that only with a low viscosity zone will the flow due to fracture zones not disturb the average depth versus age relationships.

We have also applied the model to a numerical study of the effect of a low viscosity zone in the uppermost mantle on the onset and surface expression of convective instabilities in the cooling oceanic plates. We find that the onset and magnitude of the geoid, topography and heat flow anomalies produced by these instabilities are very sensitive to the viscosity contrast and the Rayleigh number, and that the thickness of the low viscosity zone is constrained by the wavelength of the observables. If the Rayleigh number of the low viscosity zone exceeds a critical value then the convection will be confined to the low viscosity zone for a period which depends on the viscosity contrast and the Rayleigh number. The small scale convection will eventually decay into longer wavelength convection which extends throughout the upper mantle, so 
that the small scale convective signal will eventually be succeeded by a longer wavelength signal. We compare our model to the small scale geoid and topography anomalies observed in the southeast Pacific. The magnitude (0.50-0.80 $\mathrm{m}$ in geoid and $250 \mathrm{~m}$ in topography), early onset time (5-10 m.y.) and lifetime (over $40 \mathrm{~m} . \mathrm{y}_{\text {.) }}$ of these anomalies suggest a large viscosity contrast of greater than two orders of magnitude. The trend to longer wavelengths also suggests a high Rayleigh number of near or over $10^{6}$ and their original 150-250 $\mathrm{km}$ wavelength indicates a low viscosity zone of 75$125 \mathrm{~km}$ thickness. We have found that the presence of such small scale convection does not disturb the slope of the depth-age curve but elevates it by up to $250 \mathrm{~m}$, and it is not until the onset of long wavelength convection that the depth-age curves radically depart from a cooling halfspace model. In the Pacific, the depth-age curve is slightly elevated in the region where small scale convection is observed and it does not depart from a halfspace cooling model until an age of $70 \mathrm{~m} . \mathrm{y}$.. Models that produce the small scale anomalies predict a departure time between 55 and $65 \mathrm{~m} . \mathrm{y}$.. These calculations also predict an asymptotic heat flow on old ocean floor which is higher than the plate model and between 50 and $55 \mathrm{mw} / \mathrm{m}^{2}$. This value agrees with measurements of heat flow on old seafloor in the Atlantic.

In conclusion, we prefer an approximate model for the viscosity structure of the upper mantle which initially has a $125 \mathrm{~km}$ thick low viscosity zone that represents a viscosity contrast of two orders of magnitude. The viscosity contrast decreases as the plate ages to one order of magnitude or less by $130 \mathrm{~m} . \mathrm{y}$., and the low viscosity zone may also thicken with age. Finally, the Rayleigh number of the upper mantle is at least $10^{5}$ and may be as large as $10^{7}$. With this model, the evolution of the surface plates would initially involve small scale convection which is driven by shear coupling to instabilities downstream and to small scale convection associated with fracture zones. This convective flow would begin at close to $5 \mathrm{~m} . \mathrm{y}$. and remain confined to the low viscosity zone until nearly $40 \mathrm{~m} . \mathrm{y}$.. As this convective flow cools the upper mantle beneath the low viscosity zone, longer wavelength convection begins throughout the upper (or whole) mantle, and the heat transport from the longer wavelength convection flattens the depth-age curve and may form swells.

Thesis Supervisor:

Barry Parsons

Reader of Geodesy, Oxford University UK 


\section{ACKNOWLEDGMENTS}

First, and foremost, I would like to thank my advisor, Barry Parsons, for his dedication to teaching me to be a scientist. One day, before I started to work on this thesis, Barry said: "The only thing that I can teach you which you need to learn is PATIENCE." Well, rather uncharacteristically, Barry was wrong in that I learned many things other than that from him. However, he was right that I needed more patience. Unfortunately, after the three or four years that I have worked with Barry, I cannot say that I have fully acquired this desirable personality trait, but I can hope that the lessons that I have learned about patience and the quality and completeness of good scientific research will stay with me and my work.

Besides my advisor many other people contributed to the completion of this work. Steve Daly taught me the ins and outs of the convection code and the art of convection modelling. Mavis Driscoll familiarized me with the geoid anomalies and geoid-age relationships at fracture zones, and told me about many tips to make the thesis process easier. Marcia McNutt introduced me to the world of research and academia, and has always afterwards provided me with fresh views on my research and those of others. Tom Jordan, Joe Pedlosky and Marc Parmentier thoroughly read and commented on this thesis, and gave insights into the physical processes involved in these problems. Ros Binks, Linda Meinke and Dave Krowitz helped me tame the computers (the 
monsters). Tina, Inna, Nina, Lyle, and Carol (Raimey) provided a buffer from the innumerable administrative hassles at MIT and Oxford. And, Bill Brace funded me in the last few months of my graduate tenure. At the end of each chapter, specific acknowledgments are also given. My friends at MIT and Oxford, who were there to talk, to watch basketball, to play bridge or just to hang out, are too many to name. I hope they know that I hold them very dear and each for their own characteristics. However, a special thanks goes to my office-mate for 3-4 years, Karen Fischer, for without her I might have lost my sanity. I would also like to thank everyone on the eighth floor at MIT and everyone in the computer room/graduate student offices at oxford for providing an excellent place to develop and finish this thesis.

My family also deserves a lot of credit. My mother did everything that she could from seattle to make my life as easy and fun as possible in Boston. My brother and his wife provided an infinite amount of support, especially during my first years in Boston while they lived in the suburbs up north. And, my sisters (Chris and Laura) and their spouses (Randy and Mark), and Aunt Jean, Uncle Wes and my cousins were always there to talk and to visit. I couldn't ask for a better family.

Finally, an especially important thanks goes to Alan Leinbach, for it is with him that I have shared the agony and exultation that marked the creation of this thesis. His 
love and support helped me through the worst of the times and, thank God, he knows how to have fun too.

I dedicate this thesis to my parents. To my father, who died when I was 16 , but had already given me the wisdom and love of a lifetime; and, to my mother, whose wisdom and love I cherish every day. 
TABLE OF CONTENTS

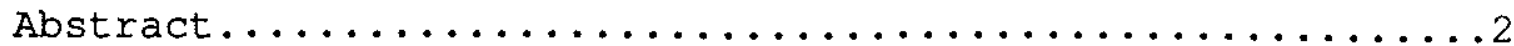

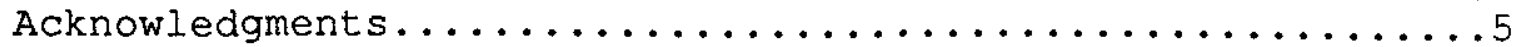

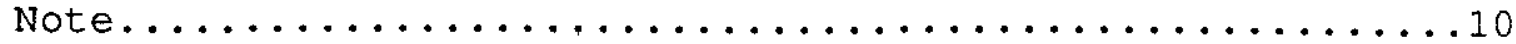

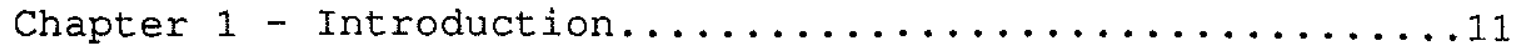

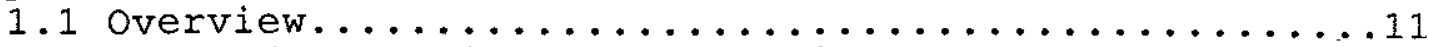

1.2 Forward Modeling of Convective Flow in the Mantle.18

1.3 Numerical Modelling of High Rayleigh Number

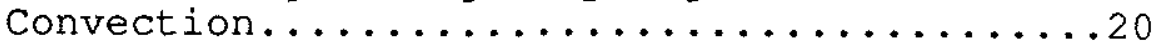

1.4 The Viscosity Structure of the Upper Mantle......22

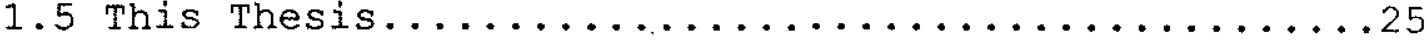

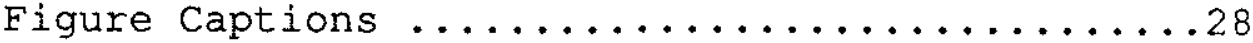

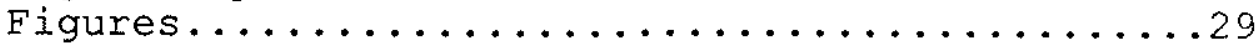

Chapter 2 - The Apparent Compensation of Mid-Plate Swells.33

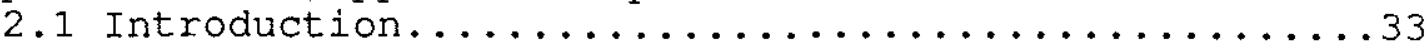

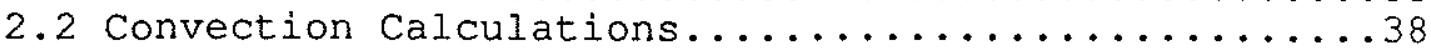

2.3 Topography, Geoid \& Gravity Response Functions.....43

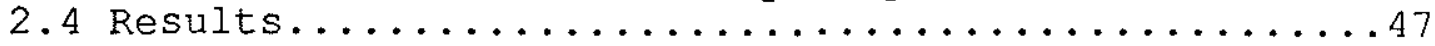

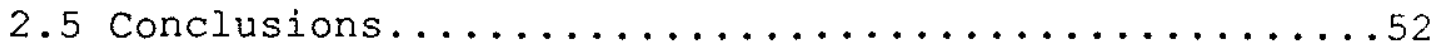

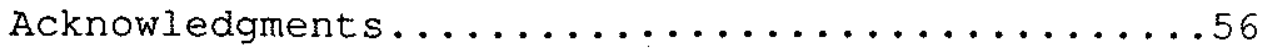

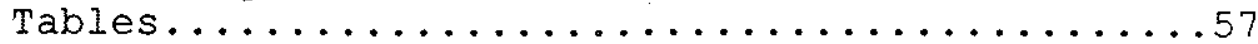

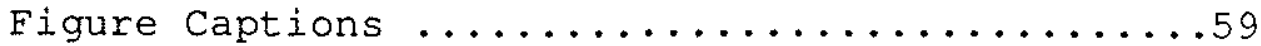

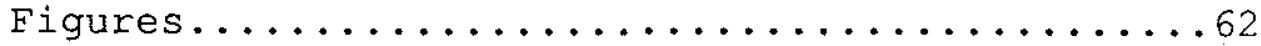

Chapter 3 - The Formation of Mid-Plate Swells.........74

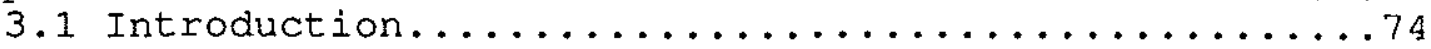

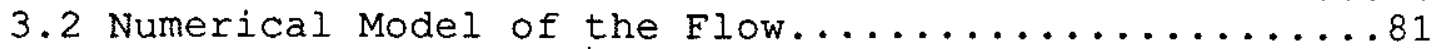

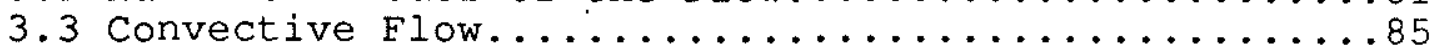

3.4 Calculation of the Surface Expression...........88

3.4.1 Topography, Gravity and Geoid..........89

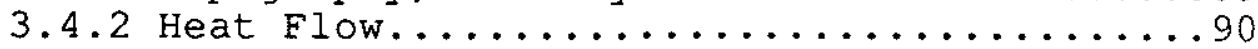

3.4 .3 Depth of Compensation..............90

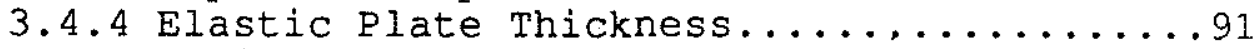

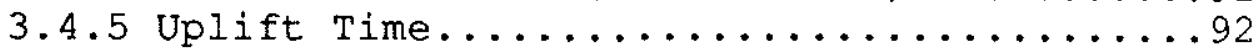

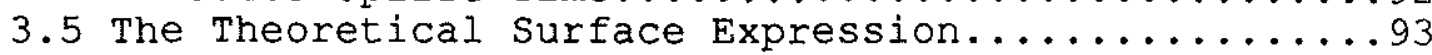

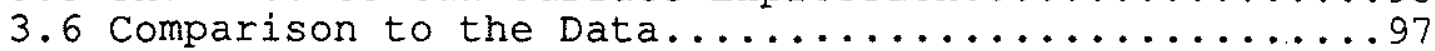

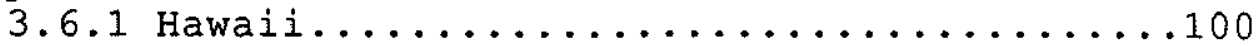

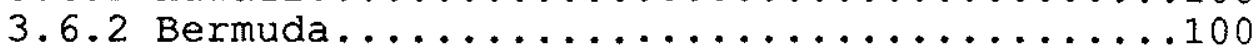

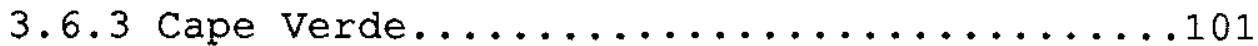

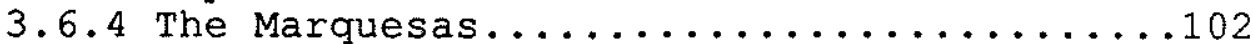

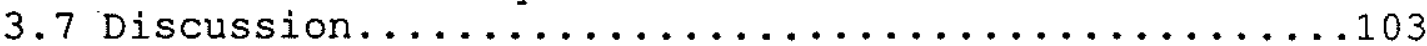

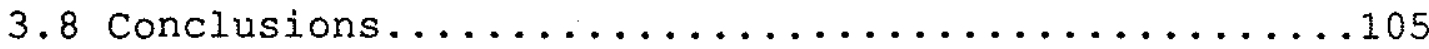

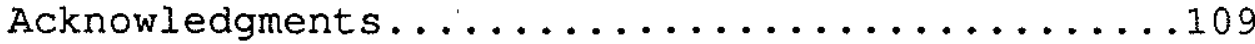

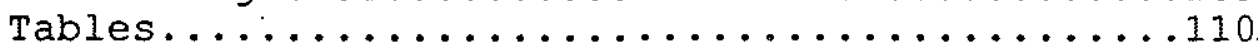

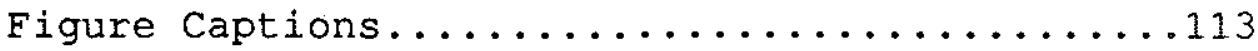

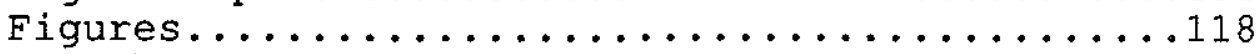


Chapter 4 - The Mantle Flow \& Geoid Anomalies at

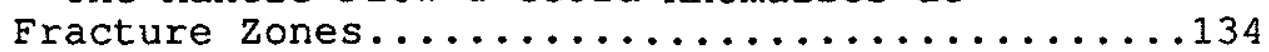

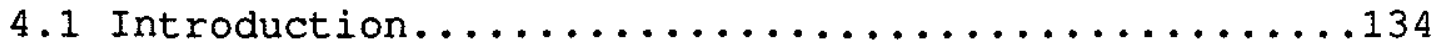

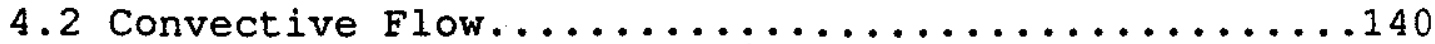

4.3 Calculation of the Geoid \& Topography Anomalies...152

4.4 The Geoid Anomaly and the Geoid Slope with Age....155

4.5 Comparison to the Udintsev Fracture Zone..........161

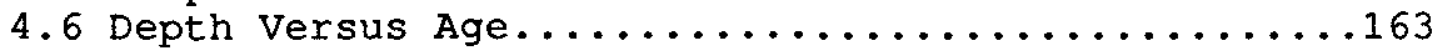

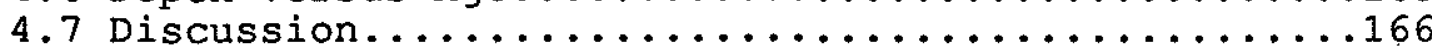

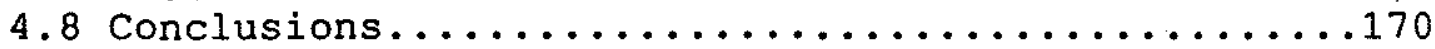

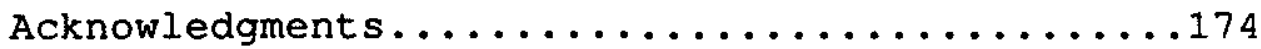

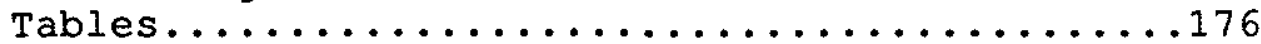

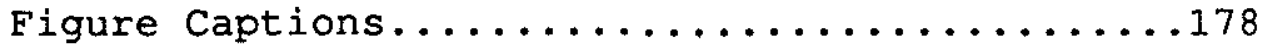

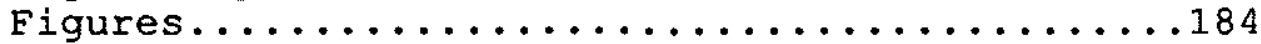

Chapter 5 - Instabilities in the Cooling Oceanic Plates..214

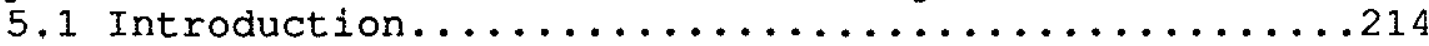

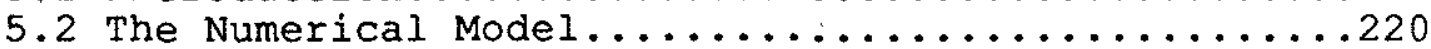

5.3 Convection Induced by Cooling from Above.........226

5.4 Geoid, Topography, Gravity and Heat Flow.........234

5.5 Depth-Age, Geoid-Age and Heat Flow-Age...........239

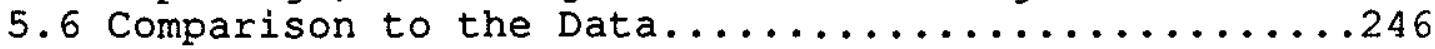

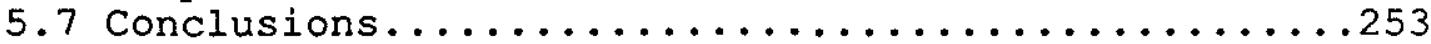

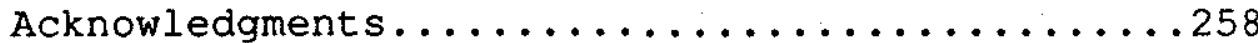

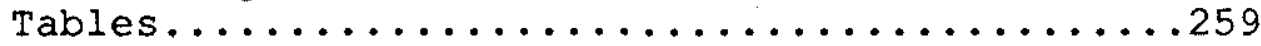

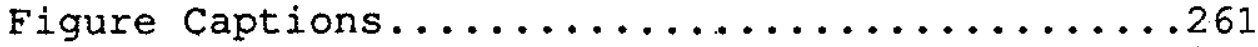

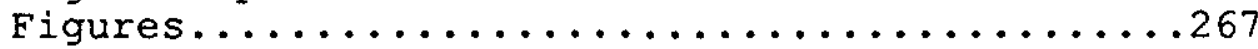

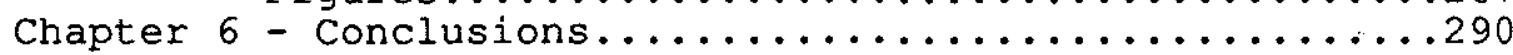

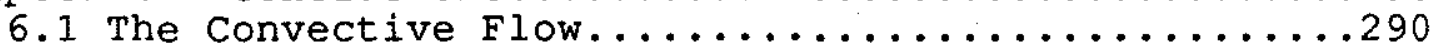

6.2 The Topographic, Geoid, Gravity \& Heat Flow

Response to the Convective Temperature

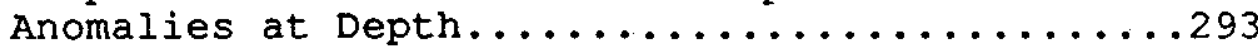

6.3 Constraints on the viscosity structure of the

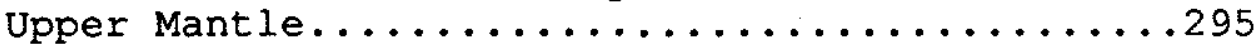

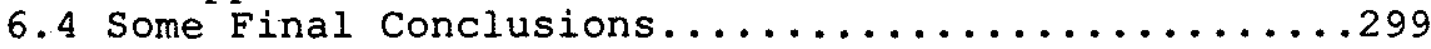

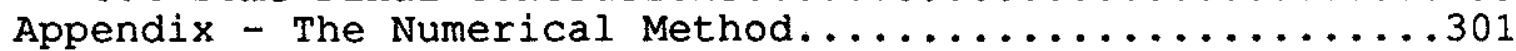

A.1 Stokes Flow Formulation for Finite Elements.......301

A.2 Penalty Function Formulation for the Pressure....301

A.3 Matrix Formulation of the Stokes Flow Problem....304

A.4 Resolution as a Function of Grid Size..........306

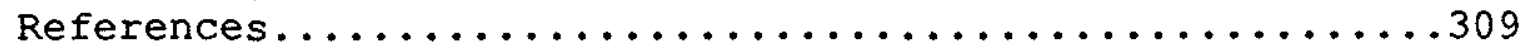

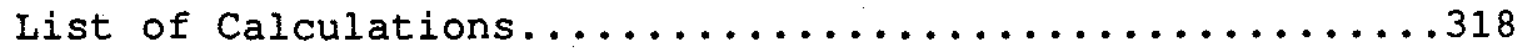


Note

Each chapter was written to stand alone as a description of one or all of the problems considered in this thesis. The reader may then pick and choose the chapters that he/she would like to read, and expect to fully understand the problem and its resolution. However, a certain amount of repetition must then occur between the chapters, and I hope that the more comprehensive reader will forgive the extra explanation. 
Chapter 1: INTRODUCTION

\subsection{Overview}

The Earth's mantle deforms in response to a number of driving forces including earthquakes and glacial loads. However, the dominant form of deformation in the mantle is due to convective currents, driven by the residual heat from the formation of the Earth and the decay of radioactive elements (Turcotte and Oxburgh, 1967; Knopoff, 1967; McKenzie, 1969; Richter, 1973; McKenzie et al., 1974; Yuen et al., 1981). The discovery of viscous flow in the mantle grew out of the plate tectonic revolution in the earth sciences during the mid-1960's. In particular, the plate tectonic description of the deformation of the surface of the Earth established that seafloor is continually destroyed at subduction zones and replenished at mid-ocean ridges, and consequently that the mantle must flow to accommodate this transport of material (Turcotte and Oxburgh, 1967; Richter and McKenzie, 1978).

As developments in the plate tectonic hypothesis continued, the driving force for the movement of the oceanic plates was presumed to be forces exerted on the plates by convection in the mantle (Turcotte and Oxburgh, 1967; Peltier, 1974; Yuen et al., 1981). Under this assumption, the plates are the visible expression of the top boundary of a convection cell and the convective flow represented by the plates is the dominant large scale flow pattern in the 
mantle. However, the connection between the convective flow in the mantle and the motions of the plates at long wavelengths remains unclear (Forsyth and Uyeda, 1975). In particular, the description of the oceanic plates as a conductively cooling thermal boundary layer has proved inadequate in explaining a number of observed features of the plates (Parsons and Sclater, 1977; Detrick, 1981).

In this thesis, we explore models of the formation of the most prominent of these features: mid-plate swells (Chapters 2, 3 and 5), the geoid anomalies and geoid-age relationships at fracture zones (Chapter 4 ), the flattening of the depth-age and the heat flow-age curves (Chapter 5), and the recent observations of short wavelength geoid anomalies in the SEASAT data over the Central Pacific and Indian oceans (Chapter 5). The important similarity between these features is that they each require a thermal source or non-conductive cooling at depth. We briefly present them here in order of decreasing wavelength.

At young ages in the oceanic plates, conductive cooling mechanisms in the mantle can produce the observed depth-age and heat flow-age relationships (Parsons and Sclater, 1977). In Figure 1.1, we have drawn the mean depth data as a function of the square root of time in the North Pacific and North Atlantic (from Parsons and Sclater, 1977). Eor comparison, we have also drawn the depth-age curve that is predicted by conductive cooling in the mantle. At ages above $70 \mathrm{~m} . \mathrm{y} .$, the depth-age curve departs from the 
conductive cooling model, and the conductive cooling mechanism appears to be retarded. Evidence also suggests that the heat flow-age curve flattens (Sclater and Francheteau, 1970; Parsons and Sclater, 1977). Estimates of the initial time of flattening in the heat flow-age relationship have until recently been close to $120 \mathrm{~m} . \mathrm{y}$. (Parsons and Sclater, 1977), but recent evidence suggests that it may flatten earlier (Detrick et al., 1986; Louden et al., 1987). To produce these deviations in the depth-age and heat flow-age curves, a thermal source is required to supply heat to the base of the plate, over and above that supplied by conductive mechanisms. Parsons and McKenzie (1978) hypothesize that the flattening of the depth-age and heat flow-age relationships may be due to heat transport from convective instabilities in the cooling oceanic plates. In fact, Parsons and McKenzie (1978) have showed that oceanic plates can go convectively unstable in the region under the rigid portion of the plate in their lifetimes, and Houseman and McKenzie (1982) have also showed that these instabilities would flatten the depth-age curve.

Mid-plate swells are regions of the seafloor associated with geoid, gravity, topography and heat flow highs in an intermediate (1000-2000 km) wavelength range (Dietz and Menard, 1953; Crough, 1978; Detrick and Crough, 1978). In Figure 1.2, we show the geoid field over the Bermuda swell. The characteristics of swells are described in greater detail in Chapters 2 and 3 . Since the heat flow is elevated 
underneath swells in comparison to the surrounding plate, however, a thermal source must exist below the surface. Such a thermal anomaly can also explain the geoid, gravity and topography anomalies (Dietz and Menard, 1953; Crough, 1978; Detrick and Crough, 1978; Von Herzen et al., 1982; McNutt and Shure, 1986). Many researchers believe that this thermal anomaly is created by an upwelling in the mantle and that mid-plate swells are the surface expression of concentrated upwellings in a convective flow (Dietz and Menard, 1953; McKenzie et al., 1980).

Other observed but currently unexplained features are the geoid slope-age relationships that are inferred from the geoid anomalies at fracture zones. Fracture zones are boundaries between plate segments of different age. In the conductive cooling models of the plates, since each plate segment has undergone a different amount of cooling, the depth and geoid heights differ across the fracture zone and, in both depth and geoid, the anomalies contain a step at the fracture zone. Recent data which constrains the geoid-age relationship at fracture zones shows that steps are evident in the geoid anomalies (see Figure 4.17) but that they do not evolve, as the plate segments cool, in accordance with either the halfspace or thermal plate models (see Figure 4.18). Furthermore, the observed geoid slope-age relationships vary from fracture zone to fracture zone (Cazenave et al., 1984). For example, entirely different geoid slope-age relationships are found at the Mendocino, 
Eltanin, Udintsev and Falkland-Agulhas fracture zones (Sandwell and Schubert, 1981; Detrick, 1982; Cazenave et al., 1984; Driscoll and Parsons, 1987; Freedman and Parsons, 1987). The geoid slope-age relationship measures long wavelength changes in the geoid field at fracture zones. Therefore, the deviations from the conductive cooling models that are observed in the geoid slope-age relationships at fracture zones and the variability between fracture zones must be due to sources that are not confined to an area near each fracture zone, but that affect the broad regions around them. Since the temperature gradient between lithosphere of differing age across the fracture zone will drive convection in a viscous Earth, the variability in the geoid anomalies and the geoid-age relationships at fracture zones may also be explained by the convective flow underneath them (Craig and McKenzie, 1986).

The final unexplained feature of the oceanic plates was discovered in the SEASAT data set. The SEASAT satellite samples the surface height of the oceans which closely approximates the Earth's geoid (Talwani, 1970). This data set has an accuracy of better than $10 \mathrm{~cm}$ and a ocean-wide coverage with a resolution of near $100 \mathrm{~km}$ between tracks (Tapley et al., 1982). In the Central Pacific at ages of 5$40 \mathrm{~m} \cdot \mathrm{y}$. and in the Central Indian Ocean, 150-250 km wavelength geoid anomalies with amplitudes of $0.50-0.80 \mathrm{~m}$ have been observed, with a possible trend towards longer wavelengths with age (Haxby and Weissel, 1986; Cazenave et 
al., 1987). The anomalies are lineated parallel to the motion of the Pacific plate (Haxby and Weissel, 1986). A shipboard study along several SEASAT tracks over young ocean floor in the Central Pacific found that the geoid anomaly was also associated with a topographic anomaly of close to $250 \mathrm{~m}$ (pers. comm. Parsons, 1987). The shiptracks and two sets of the gravity and topography lines from this oceanic cruise are drawn in Figure 1.3. In line 1 (Figure 1.3(a)), just west of the East Pacific Rise, no signal is present. By line 2, however, which is parallel to the East Pacific Rise on $6 \mathrm{~m} . \mathrm{y}$. old crust, a 150-250 km wavelength signal is apparent. The signal in the gravity line correlates with the signal in the topography line, and its magnitude, 10-15 mgals, confirms the geoid estimates of the anomaly. Since the geoid and topography anomalies correlate between tracks in a direction that is oblique to the fracture zone trend but parallel to plate motion, they most likely must also be due to a source beneath the plates (Haxby and Weissel, 1986). Buck and Parmentier (1986) and Haxby and Weissel (1986) propose that these anomalies are the surface expression of instabilities in the cooling oceanic plates which were originally hypothesized to produce the flattening of the depth-age and heat flow-age curves. However, an immediate problem is apparent with this explanation, since the small scale anomalies are evident at $5-40 \mathrm{~m} . \mathrm{y}$. In age and the flattening of the depth-age curve does not occur 
until $70 \mathrm{~m} \cdot \mathrm{y}$. Nevertheless, this explanation seems Iikely given the nature of the lineated anomalies.

In summary, each of these features requires a thermal source at depths beneath the rigid portion of the plates. Two processes produce thermal anomalies in the mantle: enhanced radioactivity and thermal convection (Roberts, 1967; Runcorn, 1969; MCKenzie et al., 1974; Jarvis and Peltier, 1982). However, in an explanation of each of the above features, a convective source seems most likely. Since very different convective flows can produce the same geoid, gravity, topography and heat flow anomalies at the surface, the inversion of the data for the thermal source function is nonunique (Parker, 1977). Since the inverse problem is not well posed (at least at present), however, we can only forward model each of the problems, i.e. take a parameterized model of the mantle and vary its parameters until a good fit to the data is achieved. With this approach, there is no guarantee that the set of parameters which give the best fit to the data is unique and that the correct solution has been isolated (Backus and Gilbert, 1967; Backus and Gilbert, 1968). However, given the correct rheological structure for the mantle and the boundary conditions on the flow and the temperature that bound it, the convective flow is then determined. The inverse problem is then reduced to the determination of the nature of the rheological structure and the boundaries on flow in the mantle. Therefore, the emphasis must be placed 
on gaining an understanding of the essential physics which governs the variation of these parameters and, as more constraints are placed on the flow and on the rheological properties of the mantle by the data, a better model can be achieved.

\subsection{Forward Modeling of Convective Flow}

The first mathematical descriptions of thermal convection were developed in the late 19th century. Two of the most significant theoretical developments towards the understanding of convective flow were made by Lord Rayleigh and Osborne Reynolds. They found that, given a set of boundary conditions, any convective flow can be characterized by two nondimensional numbers which are now called the Rayleigh number (Rayleigh, 1916) and the Reynolds number. In practice, the Rayleigh number is proportional to the ratio between the time that it takes to heat a layer of fluid by conduction and the time that it takes a particle of fluid to circulate once around the convective cell. The Reynolds number represents the ratio of the inertial forces to the viscous forces. Since the Reynolds number in the Earth is very small (around 10-10), the inertial forces are negligible when compared with the viscous forces (Turcotte and Oxburgh, 1967; McKenzie, 1969; McKenzie et al., 1974). In the mantle, the Rayleigh number is the most significant parameter that governs the flow (Turcotte and Oxburgh, 1967; McKenzie, 1969; Mckenzie et al., 1974). 
Without the effects of internal heat generation included, the Rayleigh number, Ra, can be given by:

$$
\mathrm{Ra}=\mathrm{g} \alpha \Delta \mathrm{Td}^{3} / \mu \kappa
$$

where $g$ is the acceleration of gravity, $\alpha$ is the thermal expansion coefficient, $\Delta \mathrm{T}$ is the temperature difference across the fluid layer, $d$ is the depth of the fluid layer, $\mu$ is dynamic viscosity and $\boldsymbol{k}$ is thermal diffusivity. Estimates of the magnitude of the Raleigh number in the upper mantle in the literature range from $10^{6}$ to $10^{7}$, where the variation in the estimate is due to the uncertainty in the magnitude of the physical parameters, $g, \alpha, \Delta \mathrm{T}, \mathrm{d}, \mu$ and K, (McKenzie, 1967; Richter, 1973; McKenzie et al., 1974). Since the Rayleigh number can scale laboratory results to apply to mantle flow, experiments have been done that empirically, as well as theoretically, reveal the nature of Rayleigh-Bernard convective flow in a viscous fluid as its Rayleigh number increases to mantle values in three-dimensions (Busse, 1967; Busse and Whitehead, 1971). Below a critical Rayleigh number, the fluid will not convect, but transport heat by conduction. Above this critical number, which is near $10^{3}$ and depends upon the boundary conditions, the fluid convects in two dimensional cylindrical cells and arranges its "plan form" (the spatial orientation of the convective flow pattern) in response to the initial temperature disturbance. When the Rayleigh number is increased to values above $2 \times 10^{4}$, from an initial two dimensional cylindrical flow pattern, two dimensional 
cells are no longer stable and the flow becomes three dimensional. Another set of cells grows up perpendicular to them, forming an overall pattern which is called "bimodal" convection. Above a Rayleigh number of $10^{5}$, the bimodal convection pattern in turn becomes unstable and the flow assumes a "spoke" pattern. The spoke pattern is characterized by thin, intense sites of upwelling and broad, diffuse downwellings. Above this range of Rayleigh numbers, laboratory experiments with a negligible Reynolds number are very difficult (Busse and Whitehead, 1971; Busse and Whitehead, 1974; Richter and Parsons, 1975; McKenzie, 1983). Therefore, most of our knowledge of flow at these Rayleigh numbers comes from numerical models of the flow.

\subsection{Numerical Modelling of High Rayleigh Number Convection}

In 1974, Mckenzie et al. published the first numerical model of flow in the mantle at Rayleigh numbers up to $10^{6}$. Using finite difference numerical techniques and assuming that the flow was two-dimensional and that the viscosity of the fluid was constant, they confirmed the theoretical prediction that the advection of heat would occur primarily in small boundary layers at the edges of the cell and that the interior of the cell was nearly isothermal. As the Rayleigh number increased, the vigor of the convection increased and the boundary layers thinned.

Many researchers have since explored more realistic rheological models of the mantle than a uniform constant 
viscosity upper mantle. In particular, studies with a temperature and pressure dependent viscosity structure and other nonlinear rheologies have provided insights into the effects of the rheology on convective flow at high Pradt numbers (Parmentier, 1978; Yuen et al., 1981; Yuen and Fleitout, 1984; Fleitout and Yuen, 1984a; Buck and Parmentier, 1986). However, these studies are time consuming on computers and are therefore expensive, so that only a limited number of rheologies have been tested. Since we do not know the exact rheology of the mantle, the results are very difficult to apply to mantle flow.

We approach the problem differently. Instead of attempting to understand the fluid flow in the presence of a complex rheology, we try instead in one suite of calculations to fully explore the effect of only one feature which is expected from estimates of the rheology of the mantle and from observational data. Then the physical effect of that component of the model can be built upon as more complex models are studied. In this thesis, we have built upon studies of the effect of a conducting lid at the surface of the mantle, representing an oceanic plate, and have added a low viscosity zone in the uppermost mantle. Such a layer is indicated not only by theoretical results but observational results as well.

As a note, because the computer time that would be required to fully explore the flow in three dimensions is prohibitively large, these results and the results that we 
present in this thesis are limited to two dimensions. However, we know that at large Rayleigh numbers, the flow is three-dimensional as in the "spoke" pattern. With a twodimensional model, flow in and out of the plane of the calculation, the effects of three-dimensional perturbations to the flow and three-dimensional instabilities are not included. These effects may strongly affect the results and, in each of the problems that we consider, we will discuss the specific effects that we have ignored by only addressing two-dimensional flow.

\subsection{The Viscosity Structure of the Upper Mantle}

Due to the large temperature gradient from the surface to the interior of the mantle, the largest viscosity change in the mantle is at the surface of the Earth and results in the strength of the surface plates. The presence of plates, which act dynamically like conducting lids at the surface, affects the convective flow in three ways. First, since the thermal structure of the top portion of the plates is cool enough so that the plate is rigid, the mantle can flow beneath it, separate from the conducting lid. Second, the convective temperature anomalies are depressed to greater depths than in a constant viscosity model. Third, the plates absorb a portion of the temperature difference between the interior of the convection cell and the surface through conduction, so that the magnitude of the temperature anomalies decreases as the thickness of the conducting lid 
increases (Houseman and McKenzie, 1982; Jaupart and Parsons, 1985; Buck and Parmentier, 1986). Due to the last two effects, the geoid, gravity, topography and heat flow anomalies decrease as the plate thickness increases in thickness (Buck and Parmentier, 1986).

The viscosity structure of the mantle beneath the plates is not well known. What little evidence of the viscosity structure in the upper and lower mantles comes from laboratory results, inferences from seismic velocity anomalies, modelling of post-glacial rebound, and theoretical calculation of the viscosity structure based on estimates of the temperature and pressure structures in the mantle. According to each of the indicators (that would be sensitive to viscosity changes in 100-200 km layers), however, the most prominent feature in the uppermost mantle is a low viscosity zone underneath the oceanic plates (Anderson and Sammis, 1970; Solomon, 1972; Peltier, 1974; Weilandt and Knopoff, 1982; Bott, 1985). Cooper and Kohlstedt (1984) have shown with laboratory experiments on olivine that melt in the intersections between grains will cause the diffusion path length through an aggregate of these grains to decrease. Therefore, the presence of melt in the uppermost mantle would decrease its viscosity and change its deformation behavior. From calculations of partial melting in the mantle, melt production is thought to be confined to the top $200 \mathrm{~km}$ of the upper mantle (e.g. McKenzie, 1982). Moreover, seismic evidence also exists for 
a shallow layer with a small degree of melt throughout the oceanic mantle underneath the plates (Anderson and Sammis, 1970; Solomon, 1972). In particular, the most prominent seismic feature in the uppermost mantle is a low velocity zone extending from near $60 \mathrm{~km}$ to $150 \mathrm{~km}$ (Weilandt and Knopoff, 1982; Bott, 1985). Since this region is also associated with high attenuation, the most widely accepted explanation of its origin is that it contains a small fraction of melt (Anderson and Sammis, 1970). Therefore, the presence of the melt in the top $200 \mathrm{~km}$ of the mantle would create a low viscosity zone which extends from the base of the rigid portion of the plates to $200 \mathrm{~km}$. Finally, the experimentally determined exponential relationship between viscosity and temperature and pressure also predicts a low viscosity zone underneath the plates (Parmentier, 1978; Fleitout and Yuen, 1984; Buck and Parmentier, 1986). In the thermal boundary layer at the surface, the viscosity will rapidly decrease due to the large temperature gradient with depth. Then, in the adiabatic mantle the viscosity will increase due to the increase in pressure with depth. Beneath the plates and before the pressure causes the viscosity to increase to larger values, the viscosity is low, effectively creating a low viscosity zone underneath the plates (of at least one or two orders of magnitude less than the ambient mantle viscosity).

Theoretical calculations of the viscosity structure with temperature and pressure also predict that the low 
viscosity zone will decrease in magnitude and thicken with age (Buck and Parmentier, 1986). However, theoretical constraints on melting in the mantle indicate that melting will always be confined by the effects of pressure to a depth above $200 \mathrm{~km}$ in the uppermost mantle (e.g. McKenzie, 1981). Therefore, when both the temperature and pressure and the melting effects are included in the calculation of a theoretical viscosity structure, the low viscosity zone may remain close to its original thickness (above $200 \mathrm{~km}$ in depth and below the conducting lid) and viscosity contrast for longer than predicted when only the effects of temperature and pressure included. In fact, seismic evidence indicates that a low velocity zone (and, therefore, a region of partial melt) is measurable in the uppermost mantle, above 200-400 km, throughout much of the oceanic mantle (Weilandt and Knopoff, 1982).

\subsection{This Thesis}

In this thesis, we have explored the effect of a low viscosity zone on mantle flow and its geoid, gravity, topography and heat flow expression at the surface of the Earth. In particular, we have modelled the anomalous features that were described in section 1.1 . We use a finite element numerical method to model the flow with a simple three layer viscosity structure for the upper mantle (Hughes et al., 1979; Daly and Raefsky, 1985). The numerical method is discussed in detail in the appendix. 
The viscosity model consists of a conducting lid overlying a low viscosity zone which in turn overlies a constant viscosity layer. The simplicity of the model allows us to explore a number of models where we vary the layer thicknesses, the viscosity contrast (the ratio of the viscosity contrast in the top layer, $\mu_{t}$, to that in the bottom layer: $\mu_{t}\left(\mu_{b}\right)$ and the Rayleigh number of the fluid. To calculate the gravity, gẹoid, topography and heat flow anomalies at the surface that result from the flow, we use a Green's function method (Parsons and Daly, 1983).

We have found that with the addition of the low viscosity zone the convective model not only qualitatively fits the observed anomalous features in the oceanic plates but quantitatively fits them as well. The convective models of mid-plate swells (Chapter 2 and 3), of fracture zones (Chapter 4) and of the stability of the cooling oceanic plates (Chapter 5) are all very sensitive to a shallow low viscosity zone. With our model, by specifying only the thermal boundary conditions and the three layered viscosity structure, we are able to produce a complete model for the formation of mid-plate swells, in which both the surface anomalies and the origin of the thermal source was explained. The same model (with the same range in the parameters which describe the viscosity structure) also predicts the geoid slope-age and depth-age behavior at fracture zones, the origin of the small scale anomalies in the Central Pacific and Indian oceans, and the flattening of 
the depth-age and heat flow-age curves. Therefore, we are able to place significant constraints on the viscosity contrast and the thickness of the low viscosity zone, which are consistent with theoretical estimates of the mantle viscosities. In particular, the viscosity contrast was constrained to be greater than 0.1 ; the thickness of the low viscosity zone was determined to be $75-125 \mathrm{~km}$. With the addition of a low viscosity zone, therefore, a convective model can produce many of the observed anomalous features in the oceanic plates. 


\section{Eigure Captions}

Figure 1.1: The depth-age data for the North Pacific and the North Atlantic oceans (solid line) plotted versus the square root of time. Figure taken from Parsons and Sclater (1977). The predictions of the conductive cooling model are also drawn (dashed line).

Figure 1.2: Geoid anomalies in the region of the western North Atlantic which encompasses the Bermuda swell. The anomalies are derived from the SEASAT altimeter data set by removing a GEM 9 reference field up to degree and order 10 and by smoothing the resulting geoid anomalies onto a uniform $50 \mathrm{~km}$ grid. Contour interval $1 \mathrm{~m}$. Figure taken from Detrick et al. (1986).

Eigure 1.3: (a) The shiptracks in the Central Pacific. (b) The gravity and topography data collected from lines 1 and 2 , in Figure $2(a)$. The scales are alongside the lines. In line 1, which is just west of the East Pacific Rise, there is no small wavelength signal. However, by line 2, which is on 6-10 m.y. old crust, the small wavelength signal is visible in both the gravity and topography data. 


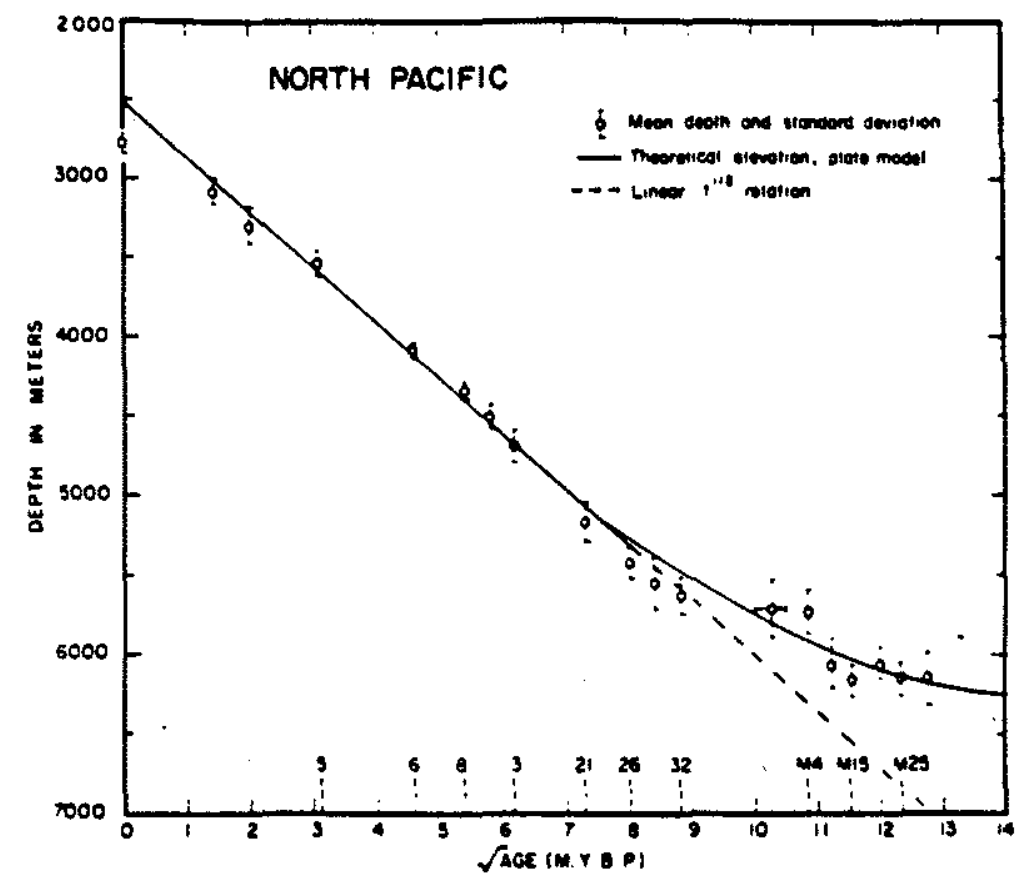

(a)

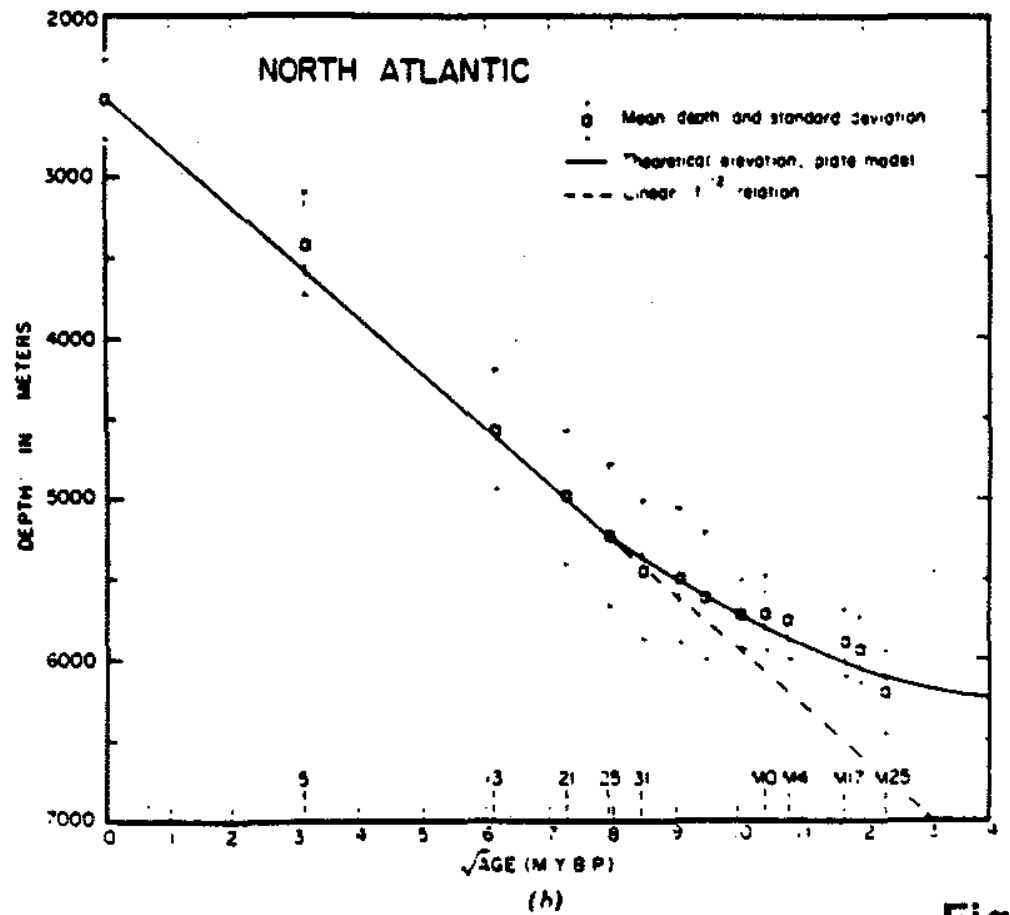

(h)

Figure 1.1 


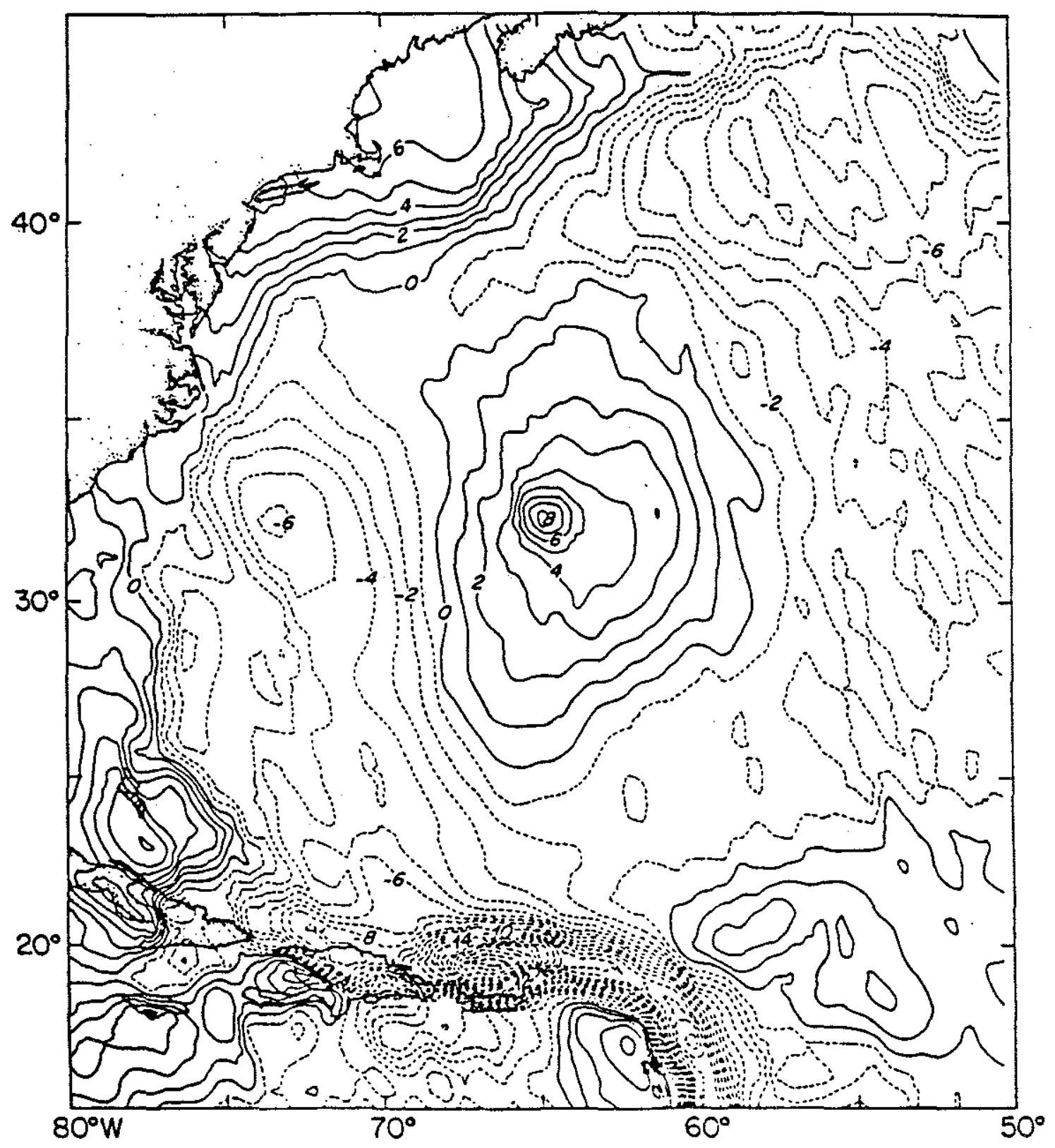

Figure 1.2 
31

S

LINE I
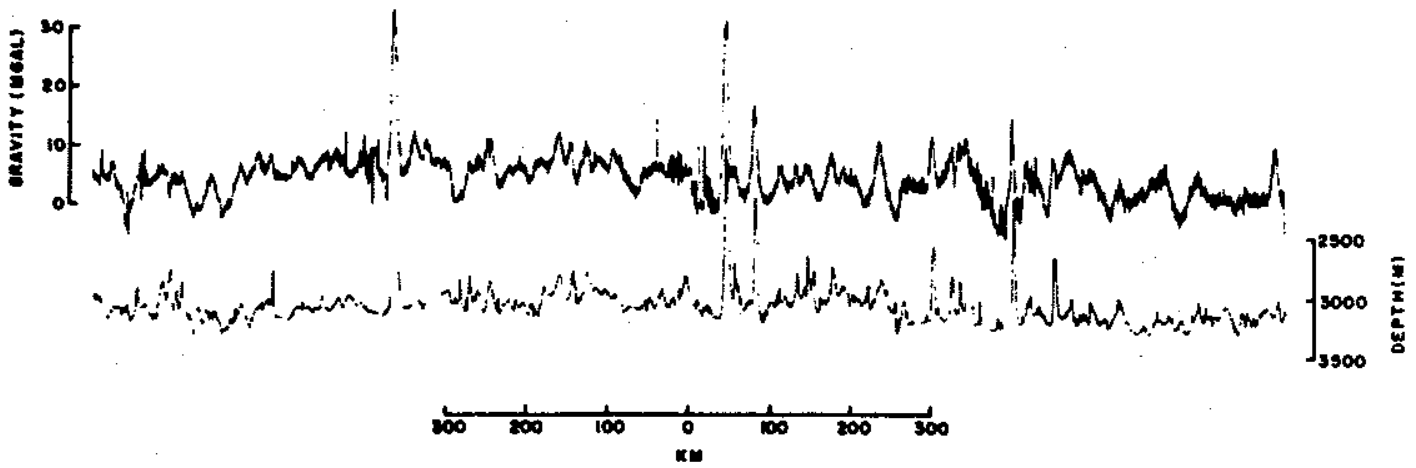

LINE 2

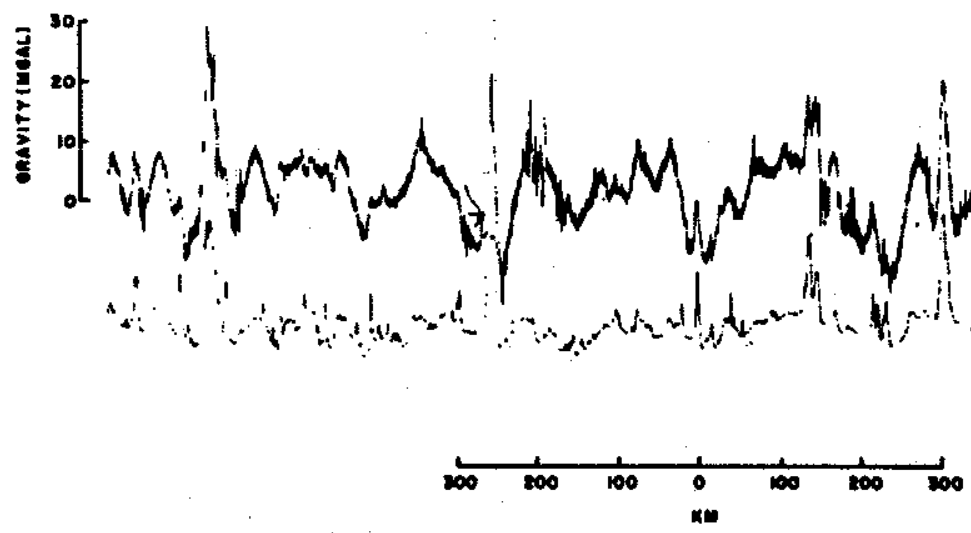

Figure 1.3a 


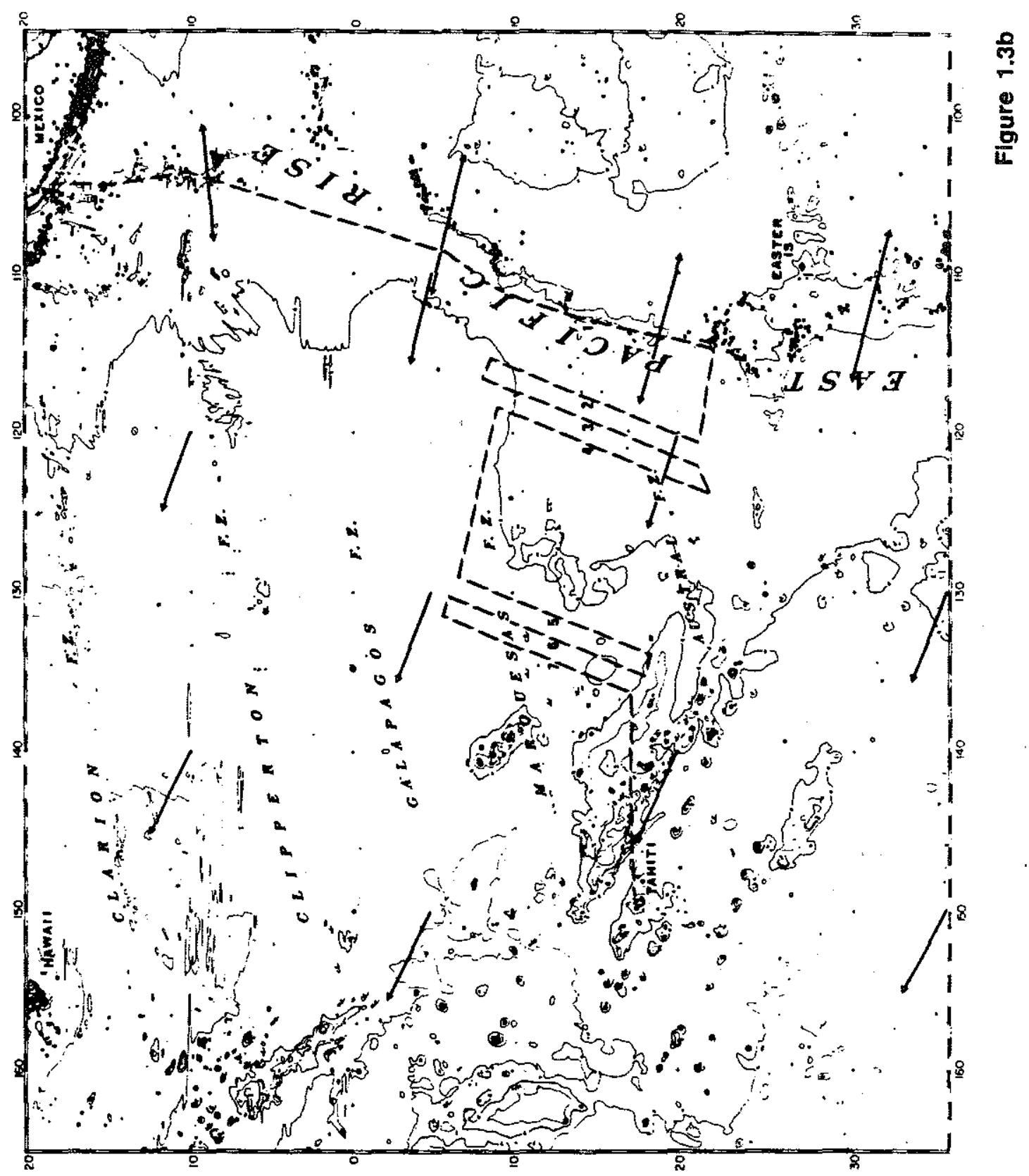


CHAPTER 2: THE APPARENT COMPENSATION OF MID-PLATE SWELLS

\subsection{Introduction}

Hot spot volcanic chains usually crest large regions of anomalously shallow seafloor, where the generally linear relationship between the long wavelength geoid and topography anomalies supports a Pratt compensation model for the uplift. Analyses of the geoid and topography anomalies at most swells, however, give a depth of compensation for the swell topography which appears shallower than the thermal plate thickness (Haxby and Turcotte, 1978; Crough, 1978). Estimates at various hotspot chains indicate depths of compensation of 60-90 km for the Hawaiian swell (Crough, 1978; McNutt and Shure, 1986), 40-70 km for the Bermuda Rise (Haxby and Turcotte, 1978), and 40-60 km for the Marquesas swell (Crough and Jarrard, 1981; Fischer et al., 1986). Because thermal cooling is thought to extend to near $125 \mathrm{~km}$ in depth underneath the seafloor surrounding these swells (Parsons and Sclater, 1977), and because, when cooled, mantle materials no longer deform so readily, these shallow compensation depths seem to pose a problem for the explanation of hotspot swells as a natural consequence of mantle and plate dynamics.

The anomalous heat flow associated with mid-plate swells argues for a thermal origin for their uplift (Von Herzen at al., 1982; Detrick et al., 1986). Detrick and Crough (1978) pointed out that, by elevating the 
temperatures underneath the Hawaiian swell, one could explain its shallow depths and subsidence. If one assumes that the plate behaves rigidly down to depths near the thermal plate thickness, as calculated from thermal cooling models (e.g. Parsons and Sclater, 1977), the elevation of the temperature structure must be accompanied by thinning of the rigid plate to a certain depth. By simply prescribing this depth, beneath which the plate is replaced by material at the ambient mantle temperature, one can fit the gravity and topography anomalies and the subsequent subsidence seen at Hawaii (Detrick and Crough, 1978; McNutt, 1984). The shallow depth of compensation is then a reflection of this thinner plate. The plate thinning that is required to produce the observed anomalies is very large, as in the case of Hawail, where the plate must be thinned from over $100 \mathrm{~km}$ in depth to just $40 \mathrm{~km}$. However, the uplift time seen at Hawaii is very short, between 5 and $8 \mathrm{~m} \cdot \mathrm{y}$. and, to produce the observed geoid and topography anomalies through conduction within this uplift time, the heat flux that is required is excessively large (Detrick and Crough, 1978; Emerman and Turcotte, 1983).

These short uplift times suggest an advective mechanism. Other models of swells describe the uplift as the surface expression of a convection cell (McKenzie et al., 1980; Detrick et al., 1986). These models in principle provide a mechanism to instigate and maintain the swell and can qualitatively produce the observed anomalies. They also 
explain the frequency and separation between swells as a consequence of the convection planform in the upper mantle but, if the thermal plate behaves rigidly down to depths corresponding to the full thermal plate thickness, they cannot produce the shallow depths of compensation. However, the viscosity of mantle material is temperature and pressure dependent, so that the plate does not act rigidly to depths corresponding to the thermal plate thickness. To a rough approximation, one can divide the plate into two parts, the boundary between which is governed by the temperature (Parsons and McKenzie, 1978). In the upper portion, the conducting lid, the temperatures are sufficiently low that the material behaves rigidly. In the lower portion of the plate where the temperatures are higher, the material can deform ductily and can participate in the mantle flow. An upwelling plume can penetrate this layer and replace it with the hotter material. The conducting lid behaves rigidly but, on a conductive time scale, thins due to heating from the plume.

Theoretical considerations and observational evidence suggest that the viscosity at depths around the thermal plate thickness can be quite small and that these low viscosities extend to depths in the mantle below the thermal plate thickness. First, theoretical calculations of the viscosity with depth, which assume an exponential temperature and pressure dependence and a temperature structure consistent with high Rayleigh number convection, 
show that the viscosity will decrease sharply to the base of the top thermal boundary layer in the mantle. In the adiabatic region immediately below it, the pressure gradient with depth will cause the viscosity to slowly rise again forming a low viscosity zone (Buck and Parmentier, 1986). second, since the presence of melt at grain boundary triple junctions may shorten the diffusive path length through a conglomerate of grains thereby enhancing the creep rate, the segregation of melt in the upper mantle may produce a layer with an effectively lower viscosity (Cooper and Kohlstedt, 1984; McKenzie, 1985). Seismic studies of the asthenosphere and upper mantle show a marked increase in attenuation and a decrease in velocity beneath the lithosphere which is often interpreted as the seismic expression of a layer with a large proportion of melt (Anderson and Sammis, 1970; Solomon, 1972). If this region is partially molten then, as predicted by the laboratory experiments of Cooper and Kohlstedt (1984), there should be changes not only in its elastic behavior but in its deformational behavior as well. Third, Craig and McKenzie (1986) have studied convection at fracture zones, which is induced by the horizontal

temperature gradient at the base of the oceanic lithosphere. Their model is two-dimensional, and consists of a fluid layer overlain by a conducting lid. They find that a thin, $150 \mathrm{~km}$ thick, Iow viscosity zone with an upper bound on the viscosity of $1.5 \times 10^{19} \mathrm{~Pa} . \mathrm{s}$ (approximately two orders of magnitude less than the average viscosity of the upper 
mantle as determined from studies of post-glacial rebound) under a conducting lid of $75 \mathrm{~km}$ can produce the general character of the geoid and topography signatures at fracture zones.

Since in any convective model for mid-plate swells, the flow reflected by the swell and the large scale circulation containing the plates are decoupled, one might argue that a low viscosity zone is inherent in convective models of midplate swells. Without decoupling, localized upwellings would be sheared by the surface flow due to the plates. This decoupling requires a region of the mantle such as a low viscosity zone whose physical properties mitigate the transmission of shear stress between the two flow regimes. Because viscosity variations in the mantle can strongly alter the topographic and gravitational response to the dynamic processes beneath the plate, convection models must include such a zone to accurately represent the formation of the swell.

To study the effect of a low viscosity zone on the convective formation of mid-plate swells, we have simplified the viscosity structure to a three layer model consisting of a conducting lid overlying a low viscosity zone which in turn overlies a constant viscosity layer extending to the base of the upper mantle (Figure 2.1). In this paper we discuss the effect of varying the thickness of the low viscosity zone, the viscosity contrast and the overall Rayleigh number (based on the bottom viscosity) on the 
gravity, topography and geoid signatures of the flow and on the inferred depth of compensation for the swell topography. In particular, for given conducting lid and low viscosity zone thicknesses, we will show that by lowering the viscosity in the layer we can produce arbitrarily small apparent depths of compensation.

\subsection{Convection Calculations}

The numerical model consists of a low viscosity layer sandwiched between a conducting lid and a constant viscosity layer, with uniform heating from below through a stress-free boundary and with a constant temperature condition on the top boundary (see Eigure 2.1). We have replaced the dimensional variables (denoted with primes) with their nondimensional counterparts through the following transformations:

$$
\begin{aligned}
& \mu^{\prime}=\mu_{O} \mu \\
& \left(x^{\prime}, z^{\prime}\right)=d(x, z) \\
& T^{\prime}=\Delta T T \\
& t^{\prime}=\left(d^{2} / k\right) t \\
& \rho^{\prime}=\rho_{O} \rho
\end{aligned}
$$

where $\mu_{0}$ is the reference kinematic viscosity, $x$ and $z$ are the horizontal and vertical coordinates respectively, $t$ is the time, $d$ is the depth of the convecting layer, $\boldsymbol{k}$ is the thermal diffusivity, $\rho_{0}$ is the reference density and $\Delta \mathrm{T}$ is the reference temperature contrast given by the constant heat flux condition: 


$$
\Delta \mathrm{T}=\mathrm{Fd} / \mathrm{K \rho _{ \textrm {o } }} \mathrm{C}_{\mathrm{p}}
$$

where $F$ is the prescribed flux and $C_{p}$ the specific heat. To scale the results, we used the values for the physical parameters given in Table 2.1 .

Omitting the primes, the equation of motion, the heat transport equation and the equation of state are given by,

$$
\begin{aligned}
& \nabla \cdot \sigma=-\mathrm{R}\left(\mathrm{T}-\mathrm{T}_{O}\right) \hat{z} \\
& \partial \mathrm{T} / \partial \mathrm{t}+\mathrm{u} \cdot \nabla \mathrm{T}=\nabla^{2} \mathrm{~T} \\
& 1-\alpha \Delta \mathrm{T}\left(\mathrm{T}-\mathrm{T}_{0}\right)=\rho
\end{aligned}
$$

where $u$ is the velocity vector, $\alpha$ is the thermal expansion coefficient, $\hat{z}$ is a vertical unit vector, $T_{0}$ is the reference temperature, $\underline{\sigma}$ is the stress tensor given by:

$$
\sigma_{i j}=-p \delta_{i j}+\mu\left(\partial u_{i} / \partial x_{j}+\partial u_{j} / \partial x_{i}\right)
$$

with $\mathrm{p}$ the pressure and $\mathrm{R}$ the Rayleigh number:

$$
R=g_{0} \alpha \Delta \mathrm{Td}^{3} / \mu_{\mathrm{O}} \kappa
$$

where $g_{0}$ is the acceleration of gravity. These equations are solved using a velocity based finite element method (Daly and Raefsky, 1985). Although the incompressibility equation:

$$
\nabla \cdot u=0
$$

is never solved explicitly, a penalty function treatment of the pressure forces incompressibility (Hughes et al., 1979). The boundary conditions on the flow are summarized in Figure 2.1 .

All of the convection calculations were run to steady state with the implicit time stepping method described in Brooks (1981). To resolve the boundary layer flow, we often 
used a nonuniform grid with double or quadruple the number of grid points in the vertical direction in the low viscosity zone. We checked the resolution and steadiness of the flow by comparing the average flux through the elements at different depths to their steady state equilibrium values. Also, due to the complex interaction of the up and downwelling plumes with the low viscosity zone, we ran the most extreme models on a uniform grid with an explicit finite difference time stepping routine for a convective overturn time to check for convergence.

In Table 2.2, we present the parameters for three suites of calculations chosen to illustrate the effect of a low viscosity zone on the convection and on the corresponding surface observables. In all of these calculations, the shear stress and vertical velocity are zero on the bottom boundary, and both components of velocity are zero at the base of the conducting lid, which is 0.125 $(75 \mathrm{~km})$ thick. In runs $1(\mathrm{a}-\mathrm{c})$ in Table 2.2 , we varied the viscosity in the low viscosity layer with a 0.21 (125 km) thick low viscosity zone and a Rayleigh number equal to $10^{5}$. In Figure 2.2, results for viscosity contrasts of $10^{0}, 10^{1}$ and $10^{2}$ are presented, and the variation of the horizontally averaged temperature and the longest wavelength component of the temperature structure with depth are given in Figure 2.3. Two essential points are illustrated by these calculations. First, since the low viscosity zone causes the local Rayleigh number of the fluid encompassing the top 
boundary layer to increase, the boundary layer thins. Second, the low viscosity zone reduces the stresses near the base of the rigid lid facilitating horizontal fluid flow. We compared these runs to ones with no conducting lid and a free top boundary. We found that this stress reduction and increased horizontal flow near the boundary caused the top boundary to look more like a free boundary to the rest of the flow and the boundary layer was correspondingly thinner. In general, an order of magnitude decrease in viscosity in the low viscosity zone will thin the boundary layer to a thickness corresponding to an order of magnitude or more increase in the local Rayleigh number. At high viscosity contrasts, the upper boundary layer can surpass its local critical Rayleigh number and become unstable, generating instabilities which sweep into the downgoing plume. The instabilities grow with a period much less than the overturn time but have a negligible effect on the longest wavelength geoid, gravity and topography signals. Due to our limited computational ability at present, however, we cannot check the resolution of this instability and do not present cases in which the Rayleigh number based on the top viscosity exceeds $10^{7}$.

In runs $2(a-c)$ in Table 2.2, we varied the Rayleigh number in the convecting layer from $10^{4}$ to $10^{6}$, with a 0.21 $(125 \mathrm{~km})$ thick low viscosity zone at a viscosity contrast of 0.1 and $a .125(75 \mathrm{~km})$ thick conducting lid (Figure 2.4). Figure 2.5 shows the mean and first harmonics of the 
temperature structures. As in Rayleigh-Bernard convection in a constant viscosity layer, the boundary layer thickness and mean temperature decrease with increasing Rayleigh number (if $\Delta \mathrm{T}$ is held constant).

In the final series of calculations (Figure 2.6), we varied the thickness of the low viscosity layer (parameter ' $a$ ' in Figure 2.1). In particular we compared two runs at a Rayleigh number of $10^{5}$ where the top layer has either a thickness of $0.08(50 \mathrm{~km})$ or of $0.5(300 \mathrm{~km})$ to the calculation already discussed where the top layer is 0.21 $(125 \mathrm{~km})$ thick. Figure 2.7 contains the mean and first harmonic temperature profiles for these runs. Initially, as the thickness of the low viscosity layer increases, the mean temperature decreases; and, at the intermediate layer thickness of $0.21(125 \mathrm{~km})$, the magnitude of the mean temperature profile is at a minimum for these runs. As the layer thickness increases further, however, the mean temperature profile returns to the profile for the very thin low viscosity layer. This nonlinear behavior in the mean temperature profile reflects the trade off between the increase in the local Rayleigh number and the effective change in the top boundary condition which can appear stress-free rather than rigid. When the low viscosity layer is thin, the boundary layer is not thinned by the change in the effective Rayleigh number of the top layer, but by the change in the apparent boundary condition. At the intermediate layer thickness, the boundary layer is thinned 
by both the change in the effective Rayleigh number and the change in the apparent boundary condition to the minimum thickness observed. At a layer thickness of half of the fluid layer depth, however, the boundary condition agains appears to be rigid, so that even though the layer is thinned by the change in the effective Rayleigh number of the top layer, the boundary layer is not as thin as in the case of the intermediate layer thickness.

For a layer $0.21(125 \mathrm{~km})$ or $0.5(300 \mathrm{~km})$, the viscosity layering also modifies the side boundary layers. It makes the downwelling plume, which originated in the low viscosity zone, much thinner than expected for the given Rayleigh number and it pinches the upwelling plume as it enters the top layer. At the intermediate layer thickness, the viscosity transition occurs very near the regions of high vorticity of the convection cell and the spread of pinch-off of the flow that occurs as the plumes hit the viscosity contrast augments the vorticity. This increase in vorticity may help to instigate the instabilities seen at high local Rayleigh numbers.

\subsection{The Topography, Geoid and Gravity Response Functions}

To calculate the gravity, geoid and topography anomalies for these calculations, we use the Green's function method formulated by Parsons and Daly (1983) in which the temperature field is decomposed into its Fourier components and, at each wavenumber, $k$, the Green's function 
response of the gravity and topography to the temperature field, the gravity and topography kernels respectively, are calculated. The surface topography kernel represents the effect of a density anomaly at a depth, $z$, on the surface topography through the transmission of normal stress. It is always positive, and varies from one at the surface to zero at the bottom boundary. On the top boundary, the topography. fully reflects a pressure perturbation at the surface so that the kernel equals one. On the bottom boundary, since the normal velocity is zero, the pressure perturbation is fully compensated by the topography on the bottom boundary and the kernel is zero. The gravity kernel, on the other hand, represents the sum of the gravitational effects of the topography on the boundaries and the density variations in the layer. Since a temperature source at a boundary produces topography with unit weighting and since the topography and temperature variations are both at the boundary, their gravitational effects cancel and the gravity kernel is always zero at the boundaries. Because the gravity kernel reflects a trade off between the effect of boundary topography and temperature variations within the layer it can change sign in the fluid layer.

The dimensional topography in the Fourier domain, $h(k)$ is given by:

$$
\begin{aligned}
h^{\prime}(k) & =\left[\rho_{O} /\left(\rho_{O}-\rho_{W}\right)\right] \alpha \Delta T d h(k) \\
& =\left[\rho_{O} /\left(\rho_{O}-\rho_{W}\right)\right] \alpha \Delta T d \int_{0}^{b} H(k, z) T(k, z) d z
\end{aligned}
$$


where $\mathrm{H}(\mathrm{k}, \mathrm{z})$ is the topography kernel and where the prime denotes the nondimensional variable. The dimensional gravity, $g(k)$, can also be written: $\mathrm{g}^{\prime}\left(\mathrm{k}^{\prime}\right)=2 \pi \mathrm{G} \rho_{\mathrm{O}} \alpha \Delta \mathrm{Td} \mathrm{g}(\mathrm{k})=2 \pi \mathrm{G} \rho_{\mathrm{O}} \alpha \Delta \mathrm{T} \alpha \int_{0}^{\mathrm{G}}(\mathrm{k}, \mathrm{z}) \mathrm{T}(\mathrm{k}, \mathrm{z}) \mathrm{dz} \quad(2.9 \mathrm{a})$ where $G(k, z)$ is the gravity kernel and $G$ is the Universal Gravitational Constant. The gravity kernel can be expressed as the sum of contributions from the surface topography, the internal density differences and the bottom boundary topography attenuated by the depth of the layer:

$G(k, z)=H(k, z)-\exp (-|k| z)+\exp (-|k|) H_{b}(k, z)$

where $\mathrm{H}_{\mathrm{b}}(k, z)$ is the kernel for the bottom boundary topography. Low viscosities in the top layer reduce the stress transmitted across the layer from buoyancy forces beneath it, so that the low viscosity zone primarily effects the first term in equation (2.9b). The low viscosity zone also alters the temperature structure in equation (2.9a), but this effect is minimal when compared to its effect on the gravity kernels. Finally, the geoid anomaly is derived from the gravity anomaly using Brun's formula:

$$
\mathrm{N}^{\prime}(\mathrm{k})=\mathrm{g}^{\prime}(\mathrm{k}) /\left|\mathrm{k}^{\prime}\right| g_{0}=\left[2 \pi \mathrm{G} \rho_{0} \alpha \Delta \mathrm{T} \mathrm{d}^{2} / \mathrm{g}_{0}\right] \mathrm{g}(\mathrm{k}) / \mathrm{k}
$$

where $\mathrm{N}$ is the dimensional geoid. For a two layer viscosity model, the kernels can be calculated analytically (Daly et al., 1984). For a general variable viscosity structure, however, we must numerically calculate them using a predictor-corrector algorithm.

In Figure 2.8, we have drawn the gravity and topography kernels corresponding to runs $1(\mathrm{a}-\mathrm{c})$ with a $0.125(75 \mathrm{~km})$ 
conducting Iid and a $0.21(125 \mathrm{~km})$ low viscosity zone. In the lid we have set the viscosity to $10^{3}$, which effectively mimics rigid behavior. The low viscosity varies from 1.0 to 0.01 . As the viscosity contrast increases, the low viscosity zone damps the surface topography kernels at depth and their power is concentrated in the top portions of the model. The surface observables are dominated by the $k=\pi$ wavenumber, and can be well approximated by the effect of this harmonic alone. To calculate the observables, we have obtained the kernels for each of the runs out to wavenumber $k=8 \pi$. For the shorter wavelengths (less than $\lambda=0.25$ ), we have approximated the kernels by those for a two layer structure of a rigid lid overlying a constant viscosity zone. Since the surface topography kernels are effectively zero before the base of the low viscosity zone, they are not affected by the viscosity jump. At the wavelength, $\lambda=2$, the topography kernels are effectively zero at the base of the low viscosity zone for a viscosity contrast of two orders of magnitude. By this viscosity contrast, therefore, the topographic response to the underlying convection is limited to depths corresponding to the conducting Iid and the low viscosity zone. The gravity and geoid kernels, on the other hand, become negative at depth as the viscosity contrast increases, so that the positive contributions from the shallow temperature anomalies are counteracted by those from deeper temperature variations. In the bottom layer, both the gravity and topography kernels tend quickly to zero so 
that the effect of the bottom boundary on the observables is minimized.

\subsection{Results}

In Figure 2.9, we present the gravity, geoid and topography anomalies for the central series of calculations (1a-c in Table 2.2) with the conducting lid 0.125 thick and the low viscosity layer 0.21 thick. Although not included in the previous section and figures we also performed calculations with viscosity contrasts of $0.075,0.050$ and 0.025 . In general the profiles are positive over the upwellings and negative over the downwellings, and are dominated by the longer wavelengths. As the viscosity contrast increases, the shape of the topography profile remains constant while its magnitude decreases. The gravity and geoid profiles follow the same behaviour until a critical viscosity contrast near 0.075 where the gravity kernel of the longest wavelength which dominates the observables $(k=\pi)$ becomes negative at depth. At this and higher viscosity contrasts, the gravity and geoid profiles begin to flatten in the middle sections across the top of the box, and their peak-to-peak magnitude decreases sharply with an increase in viscosity contrast, more sharply than the peak-to-peak magnitude of the topography anomalies with the same increase in viscosity.

To calculate an apparent depth of compensation from the calculated geoid and topography profiles, we assume pratt 
compensation which is often used to calculate the compensation depth at mid-plate swells (Haxby and Turcotte, 1978; Crough, 1978). The geoid anomaly due to a topography anomaly of wavenumber $k$, which is compensated by horizontal variations in density above a depth $2 \mathrm{~d}_{\mathrm{C}}$, is given by:

$$
N^{\prime}(k)=\left[2 \pi G\left(\rho_{0}-\rho_{W}\right) d_{C} / g_{0}\right] h^{\prime}(k)
$$

for wavelengths large compared to $d_{C}$. In the limit where equation (2.11) is valid, i.e. when the compensation depth is much less than the wavelength of the geoid anomaly, then the result also holds for Airy compensation if $d_{c}$ is interpreted in that case as the depth at which the compensating masses are concentrated.

In Figure 2.10, we have drawn a sample geoid versus topography plot along with a linear relation fit by eye. Since the depth of compensation is proportional to the slope of the geoid plotted versus the topography, and since at viscosity contrasts greater than 0.075 the magnitude of the geoid anomaly decreases faster than that of the topography, the apparent depth of compensation will decrease rapidly.

In Figure 2.11, we have plotted the depth of compensation results for each of our runs. For the central series of calculations, the depth of compensation decreases uniformly with viscosity contrast until it reaches a viscosity ratio of near 0.075 where it begins to drop rapidly with viscosity contrast. The mean temperature profile and the temperature structures at the longest wavelength $k=\pi$ are shown in Figure 2.3 . The variation of 
the $k=\pi$ component of the temperature structures with depth is dominated by two peaks, one associated with each of the horizontal boundary layers. An increase in viscosity contrast leads to a relative decrease in the magnitude of the top peak (in the low viscosity zone) compared to the bottom peak in addition to a shift in the peak close to the upper boundary. At the viscosity contrast of 0.075 , the gravity kernels have become partially negative at depth augmenting the effect of the changing temperature structure and, as: the viscosity contrast increases, the gravity anomaly decreases much more rapidly than the topography anomaly. Therefore, the inferred depth of compensation can become arbitrarily small and even "negative", when the negative contribution of the gravity kernels dominates the total anomaly so that the geoid and topography anomalies become negatively correlated.

Calculating the gravity, geoid and topography anomalies for the second set of convection results in which we vary the Rayleigh number, we find that as we increase the Rayleigh number the apparent depth of compensation decreases. Since the kernels are the same for each of the calculations in this series, the depth of compensation can change only in response to the thinning of the boundary layers with the increased Rayleigh number (Figure 2.5). Although we have only three calculations to constrain the behaviour, the depth of compensation appears to decrease linearly with the logarithm of the Rayleigh number, as 
expected from the results for constant viscosity convection under a conducting lid (Parsons and Daly, 1984). The total variation for a one order of magnitude change in the Rayleigh number is about $0.013(8 \mathrm{~km})$, and is much less than that seen for an order of magnitude change in viscosity in the low viscosity layer.

If we allow the thickness of the low viscosity layer to vary, we can again compare the effects of the thinning of the top boundary layer and the damping of the topography kernels on the depth of compensation. If the low viscosity zone is thinner than the depth to the base of the top boundary layer, then the boundary layer does not thin appreciably. The kernels also are not affected much by the low viscosity zone, so that this case approximates a conducting lid overlying a constant viscosity layer with only a slight decrease in the effective depth of compensation to $0.17(102 \mathrm{~km})$. As the low viscosity zone thickens to half of the box, it encompasses the top boundary layer allowing the layer to thin in response to the change in local Rayleigh number (Figure 2.7). The kernels, however, become less concentrated at shallow depths, so that this case produces a larger depth of compensation than that for the intermediate layer thickness of 0.21 (125 km), approximately $0.15(90 \mathrm{~km})$.

With the Green's function formulation we can test whether a Pratt compensation model is appropriate for the 
compensation in these calculations. For Pratt compensation, where we have nondimensionalized the density by $\rho_{\circ} \alpha \Delta \mathrm{T}$ :

$$
N^{\prime}(k)=2 \pi G \rho_{\circ} \alpha \Delta \mathrm{Td}^{2} / g_{\circ} \int_{0}^{b} z \Delta \rho(k, z) d z
$$

where $\Delta \rho(k, z)$ equals a constant above a depth $2 d_{C}$ and zero elsewhere. Rewriting equation $(2.10)$ in the same form as equation (2.12):

$N^{\prime}(k)=2 \pi G \rho_{\circ} \alpha \Delta T d^{2} / g_{\circ} \int_{a}^{b} z[G(k, z) T(k, z) / z|k|] d z$

reduces the analysis to a comparison of $[G(k, z) T(k, z) / z|k|]$ to the gravitational mass distribution, $\Delta \rho(k, z)$. In Figure 2.12, we plot the most influential harmonic, $k=\pi$, of the effective mass distribution for viscosity contrasts between the two layers of $10^{0}, 10^{1}$ and $10^{2}$ along with that for a Pratt model which also satisfies the calculated geoid and topography. At each viscosity contrast, the Pratt compensation model overestimates the magnitude and underestimates the maximum depth of the effective density anomaly required to produce the surface observables. At higher viscosity contrasts, the change in sign of the gravity kernels produces "negative" effective masses at depth which further complicates the structure and which can not be accommodated by a Pratt model.

In summary, the apparent depth of compensation is very sensitive to the viscosity structure and, in particular, to the presence of low viscosities beneath the conducting lid. The overall Rayleigh number and the thickness of the low viscosity zone also affect the apparent depth of compensation, but to a lesser degree. Furthermore, the 
Pratt compensation model severely underestimates the complexity and overestimates the relative magnitudes of the effective masses for the geoid anomaly. In particular, density variations may extend to a depth much greater than the apparent depth of compensation and, for even constant viscosity convection under a conducting lid, the compensating mass distribution is not well approximated by Airy, Pratt of similar compensation mechanisms. Rather, the depth under which no effective mass anomalies exist cannot be well constrained without knowledge of the viscosity structure.

\subsection{Conclusions}

We assume a model for mid-plate swells where the swell topography is dynamically maintained by convection beneath a conducting lid. Using an idealized viscosity structure consisting of a low viscosity layer underneath the conducting lid and overlying a constant viscosity region, we have explored the effect of the low viscosities, the layer thickness and the Rayleigh number on the flow and on the inference of a compensation mechanism from the associated gravity, geoid and topography anomalies.

If the viscosity is allowed to decrease in the top layer, the low viscosities decrease the stress coupling between the lid and the convecting region. Also, due to the change in viscosity, the local Rayleigh number increases which thins the boundary layer and controls the magnitude of 
the mean temperature increase across the top boundary layer. If the low viscosity layer is comparable in size to the convective boundary layer, the concentration of high velocities and low stresses near the boundary causes it to appear like a free boundary. Furthermore, the low viscosities damp the response of the surface topography to the temperature variations at depth and cause the gravity and geoid kernels to change sign at a depth inside the low viscosity zone. By increasing the viscosity contrast, therefore, the inferred depth of compensation decreases. At small viscosity contrasts, the depth of compensation decreases slowly as with a simple increase in Rayleigh number in the convecting region. At greater viscosity contrasts where the gravity kernels become negative at depth for the dominant wavelengths, the geoid-topography slope flattens rapidly and the depth of compensation can be made arbitrarily small. The viscosity contrast required to match the depths of compensation seen at Hawaii, Bermuda, the Marquesas Islands and elsewhere, therefore, is less than two orders of magnitude for a layer thickness of $125 \mathrm{~km}$ and the depth of compensation is very sensitive to the exact viscosity contrast underneath the swell.

The effect of the layer thickness on the flow and on the apparent compensation mechanism is more complicated. If. the thickness of the low viscosity zone is smaller than the thermal boundary layer of the convecting cell, then the low viscosity layer has not much effect on the geometry of the 
flow. The kernels are also not much affected by the low viscosity zone. The depth of compensation is therefore similar to that of a constant viscosity region overlain by a conducting lid. As the layer thickness increases, the boundary layer thins to a minimum thickness, but the behavior of the kernels is more complicated. Since the kernels are strongly affected by the shape of the viscosity structure at depth, at greater layer thicknesses, they must tend back to the kernels of a constant viscosity layer. As the low viscosity layer thickness increases to intermediate thicknesses, therefore, the depth of compensation decreases. As the thickness increases still further, the depth of compensation tends to that of a conducting lid over a constant viscosity layer with a lower viscosity and correspondingly higher Rayleigh number. Indeed, at large layer thicknesses, the effect of the changing viscosity contrast is comparable to the effect of changing the overall Rayleigh number of the convecting layer. The viscosity and thickness of the low viscosity zone also trade off in a comparison of the overall magnitude of the surface observables.

Because the effective mass distribution for the geoid anomaly in convective models of swell formation are complex, simple compensation models cannot effectively explain the compensation mechanism. Since we can evaluate the distribution exactly from our numerical results, we have tested the assumptions that are used to calculate 
compensating density distributions for hot spot swells. The Pratt compensating mass distribution used by Haxby and Turcotte (1978) and Crough (1978) severely underestimates the depth and the complexity of the effective mass distribution for these calculations. Without knowledge of the viscosity structure, the inferred depth of compensation from Pratt or Airy models cannot constrain the actual distribution of the compensating masses.

Even though we have scaled our calculations using a layer depth appropriate for upper mantle convection, the results will also apply to whole mantle convection. Since the topography and gravity kernels are effectively zero near the bottom of the fluid layer (see Figure 2.8) and since the temperature anomalies for the dominant wavelength peak at the top and bottom boundary layers of the convecting cell (see Figure 2.3), the topography and gravity anomalies will be determined by the temperature variations in the upper boundary layer. Moreover, because these anomalies represent integrals over depth of the temperature variations multiplied by the appropriate kernels (equations (2:8), (2.9) and (2.10)), their magnitude will depend primarily on the thickness of the top boundary layer. Since the thickness of the top boundary layer remains constant for high Rayleigh number convection as the depth of the layer is increased with the other parameters fixed, and since the temperature drop across the top boundary layer is prescribed by the value of the mean heat flux, the magnitude of the 
topography and geoid anomalies, and therefore the depth of compensation, should be insensitive to the depth of convection.

In summary, these calculations show that a simple plume model with stratified viscosity variations of less than two orders of magnitude near the surface can produce the range of depths of compensation seen at mid-plate swells. Shallow depths of compensation, therefore, cannot be used to argue against dynamic models for the formation of hot spot swells. In a subsequent paper, we will present a more complete parameter study of the flow and its applications to a model for the uplift of mid-plate swells. In particular, with a quantitative comparison of the calculations to the data at Hawaii and elsewhere, it should be possible to constrain the viscosity and thickness of the low viscosity zone.

\section{Acknowledgments}

We would like to thank Karen Fischer, Marcia McNutt, Marc Parmentier, David Sandwell and Roger Buck for helpful discussions. This work has been supported by NASA Geodynamics program grant NAG5-415, and National Science Foundation grant EAR-8306249. We would also like to thank Tom Jordan for making computing time on the Alliant FX/I available to us. 
Table 2.1

Variable

d

F

$\rho_{0}$

$\rho_{\mathrm{W}}$

$\mu_{0}$

go

K

$\alpha$

$\mathrm{C}_{\mathrm{p}}$

$\Delta \mathrm{T}$
Description

depth scale

flux on the bottom boundary

average mantle density $3300 \mathrm{~kg} / \mathrm{m}^{3}$

density of water

reference kinematic viscosity

surface gravitational $\quad 10 \mathrm{~m} / \mathrm{s}^{2}$ acceleration

average mantle thermal $10^{-6} \mathrm{~m}^{2} / \mathrm{s}$ diffusivity

average mantle thermal $2 \times 10^{-5} \circ_{C^{-1}}$ expansion coefficient

specific heat

temperature contrast across the depth of the box
$900 \mathrm{~J} / \mathrm{kg} \cdot{ }^{\circ} \mathrm{C}$

Value

$600 \mathrm{~km}$

1 H.F.U.

$1000 \mathrm{~kg} / \mathrm{m}^{3}$

$2 \times 10^{17} \mathrm{~m}^{2} / \mathrm{s}$

$8656^{\circ} \mathrm{C}$ 
Table 2.2

\begin{tabular}{|c|c|c|c|c|}
\hline RUN & $\begin{array}{l}\text { Viscosity in } \\
\text { top layer }\end{array}$ & $\begin{array}{l}\text { Top layer } \\
\text { thickness }\end{array}$ & $\begin{array}{l}\text { Rayleigh } \\
\text { number }\end{array}$ & $\begin{array}{l}\text { Elements } \\
\text { in }(x, z)\end{array}$ \\
\hline $\begin{array}{l}1 a \\
1 b \\
1 c\end{array}$ & $\begin{array}{l}1.0 \\
0.1 \\
0.01\end{array}$ & $\begin{array}{l}0.21 \\
0.21 \\
0.21\end{array}$ & $\begin{array}{l}10^{5} \\
105 \\
10^{5}\end{array}$ & $\begin{array}{l}24,27 \\
24,42 \text { *\# } \\
24,42\end{array}$ \\
\hline $\begin{array}{l}2 a \\
2 b \\
2 c\end{array}$ & $\begin{array}{l}0.1 \\
0.1 \\
0.1\end{array}$ & $\begin{array}{l}0.21 \\
0.21 \\
0.21\end{array}$ & $\begin{array}{l}104 \\
105 \\
106\end{array}$ & $\begin{array}{l}24,32^{\star} \\
24,42^{\star} \# \\
24,42^{\star}\end{array}$ \\
\hline $\begin{array}{l}3 a \\
3 b \\
3 c\end{array}$ & $\begin{array}{l}0.1 \\
0.1 \\
0.1\end{array}$ & $\begin{array}{l}0.083 \\
0.21 \\
0.5\end{array}$ & $\begin{array}{l}105 \\
105 \\
10^{5}\end{array}$ & $\begin{array}{l}24,29^{\&} \\
24,42^{\star} \# \\
24,39^{\&}\end{array}$ \\
\hline$\&-$ & $\begin{array}{l}\text { double the re } \\
\text { viscosity zo }\end{array}$ & lution in the & $z$-direction in & the low \\
\hline$\star \quad$ & $\begin{array}{l}\text { quadruple th } \\
\text { viscosity } z\end{array}$ & esolution in & the $z$-direction & in the low \\
\hline$\#-$ & $\begin{array}{l}\text { tested for } \\
\text { grid. }\end{array}$ & ergence on 49 & by 55 element, & uniform \\
\hline
\end{tabular}




\section{Fiqure Captions}

Figure 2.1: Geometry of the model: $b=1.125$ for all of the calculations in this paper. $u$ is the horizontal velocity and $w$ is the vertical velocity. $T$ is the temperature and $\sigma$ is the shear stress. ' $C$ ' is a constant.

Figure 2.2: Steady state temperature. (top) and stream function (bottom) plots for the runs: (a) 1 a ; (b) 1 b ; (c) 1c (where the numbers refer to Table 2.2). The temperature contour intervals are : (a) $190{ }^{\circ} \mathrm{C}$; (b) $160{ }^{\circ} \mathrm{C}$; (c) $140{ }^{\circ} \mathrm{C}$; and the ticks mark the boundaries of the low viscosity zone.

Eiqure 2.3: The mean and first harmonic $(k=\pi$, where $k$ is the nondimensional average number) temperature structures versus $z$ for cases: 1a (line), 1b (long dash), 1c (short dash) .

Figure 2.4: Steady state temperature (top) and stream function (bottom) plots for the convection runs: (a) $2 a$; (b) $2 \mathrm{~b} ;$ (c) $2 \mathrm{c}$ (numbers refer to Table 2.2). The temperature contour intervals are: (a) $220^{\circ} \mathrm{C}$; (b) $160{ }^{\circ} \mathrm{C}$; $140^{\circ} \mathrm{C}$; with the ticks marking boundaries of the low viscosity zone. 
Eigure 2.5: The mean and first harmonic $(k=\pi)$ temperature structures versus $z$ for cases: $2 a$ (line), $2 b$ (long dash), 2 c (short dash).

Eigure 2.6: Steady state temperature (top) and stream function (bottom) plots for the convection runs: (a) $3 a$; (b) $3 \mathrm{~b} ;(\mathrm{c}) 3 \mathrm{c}$ (numbers refer to Table 2.2). The temperature contours represent $160^{\circ} \mathrm{C}$, with the ticks on the boundaries of the low viscosity zone.

Eigure 2.7: The mean and first harmonic $(k=\pi)$ temperature structures versus $z$ for cases: $3 a$ (Iine), $3 b$ (long dash), 3c (short dash).

Eigure 2.8: The topography and gravity kernels versus $z^{\prime}$ corresponding to the viscosity structures for runs: (a) 1 a; (b) $1 \mathrm{~b} ;$ (c) 1c; where the numbers refer to Table 2.2 . (Continuous line-k= long dash $-k=2 \pi$, medium dash $-k=3 \pi$,

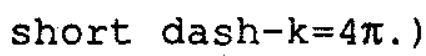

Figure 2.9: The topography, gravity and geoid signatures versus $\mathrm{x}$ for runs: $1 \mathrm{a}$ (line), 1b (long dash), lc (short dash); the runs are catalogued in Table 2.2. The scales are nondimensional, but if we use the dimensionalization in the text then: distance, $1=600 \mathrm{~km}$; topography, $1=103,872 \mathrm{~m}$; gravity $1=0.1402272 \mathrm{~m} / \mathrm{s}$; geoid, $1=8414 \mathrm{~m}$. 
Figure 2.10: An example of the best-straight-line-fit-byeye to a plot of geoid vs. topography. This plot is for case 1c of Table 2.2. The axes are dimensionalized as in the text.

Figure 2.11: The apparent depth of compensation in kilometers versus the nondimensional viscosity for the runs as in Table 2.2: 0 -cases $1(\mathrm{a}-\mathrm{c})$ plus some others with intermediate viscosities; $+-2 a ; \Delta-2 c ; \Delta-3 a ; x-3 c$.

Eigure 2.12: The first harmonic of the effective compensating density distribution versus $z^{\prime}$ for the geoid anomalies (line) with the corresponding Pratt compensation model (dash) for the cases from Table 2.2: (a) $1 \mathrm{a}$; (b) $1 \mathrm{~b}$; (c) $1 \mathrm{c}$. 


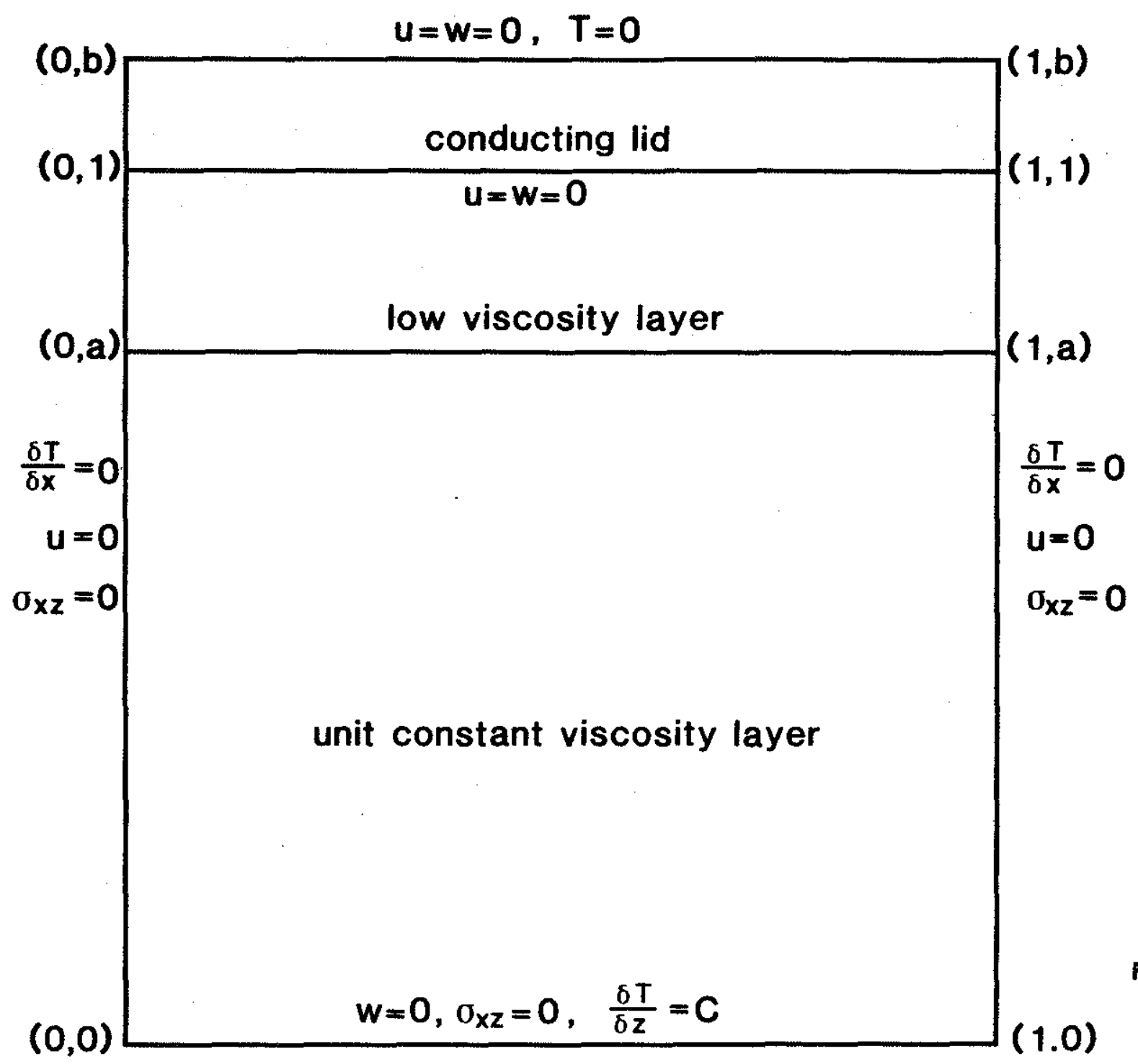

Figure 2.1 

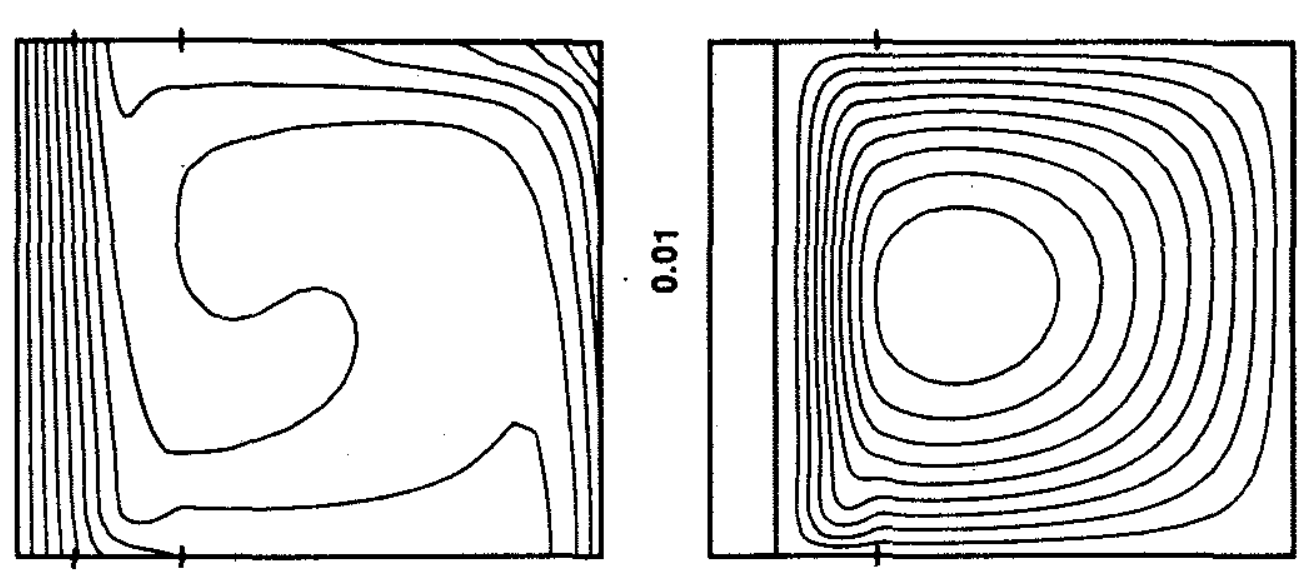

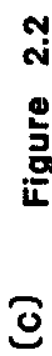
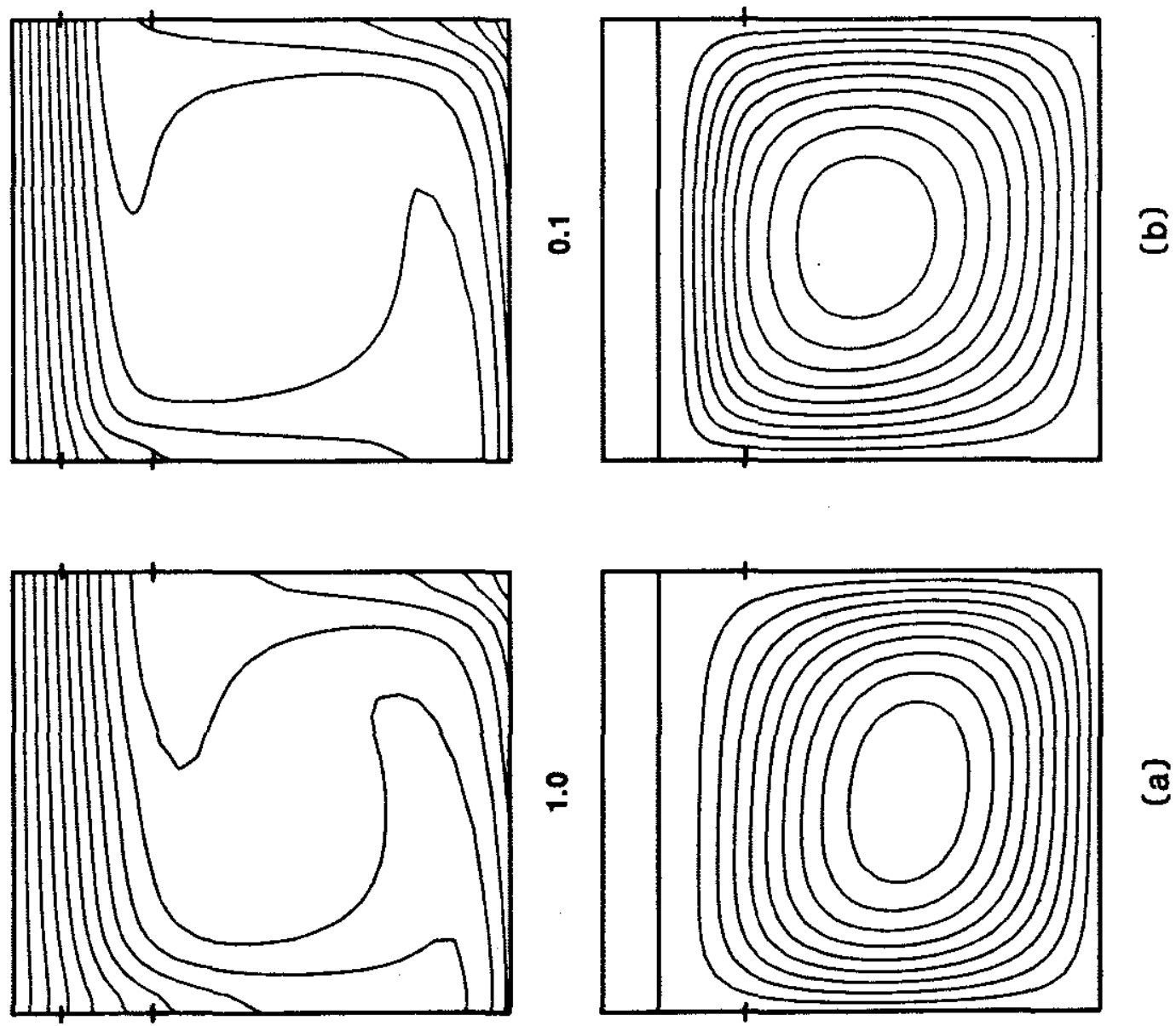


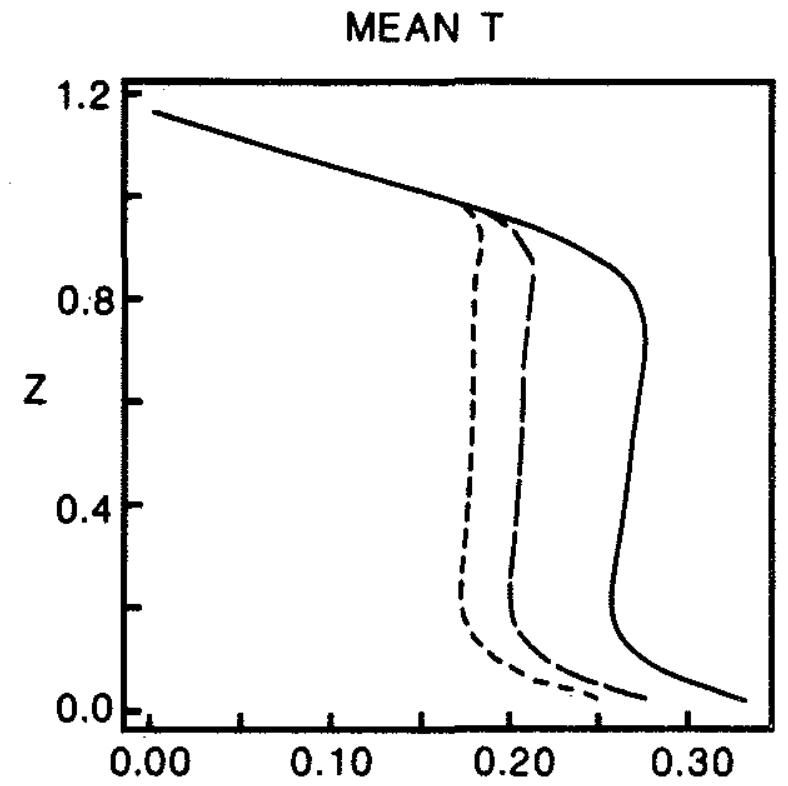

FIRST HARMONIC

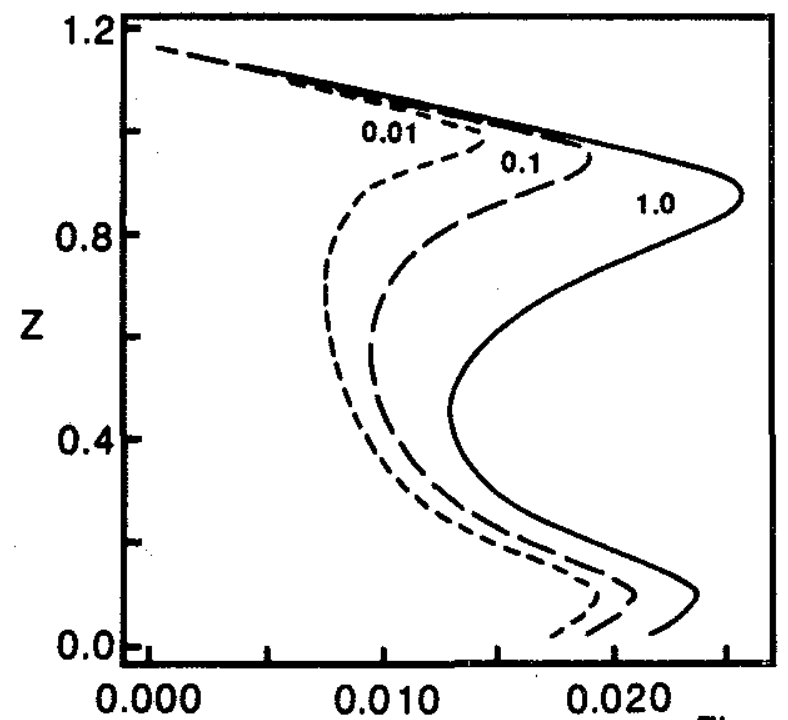

Figure 2.3 

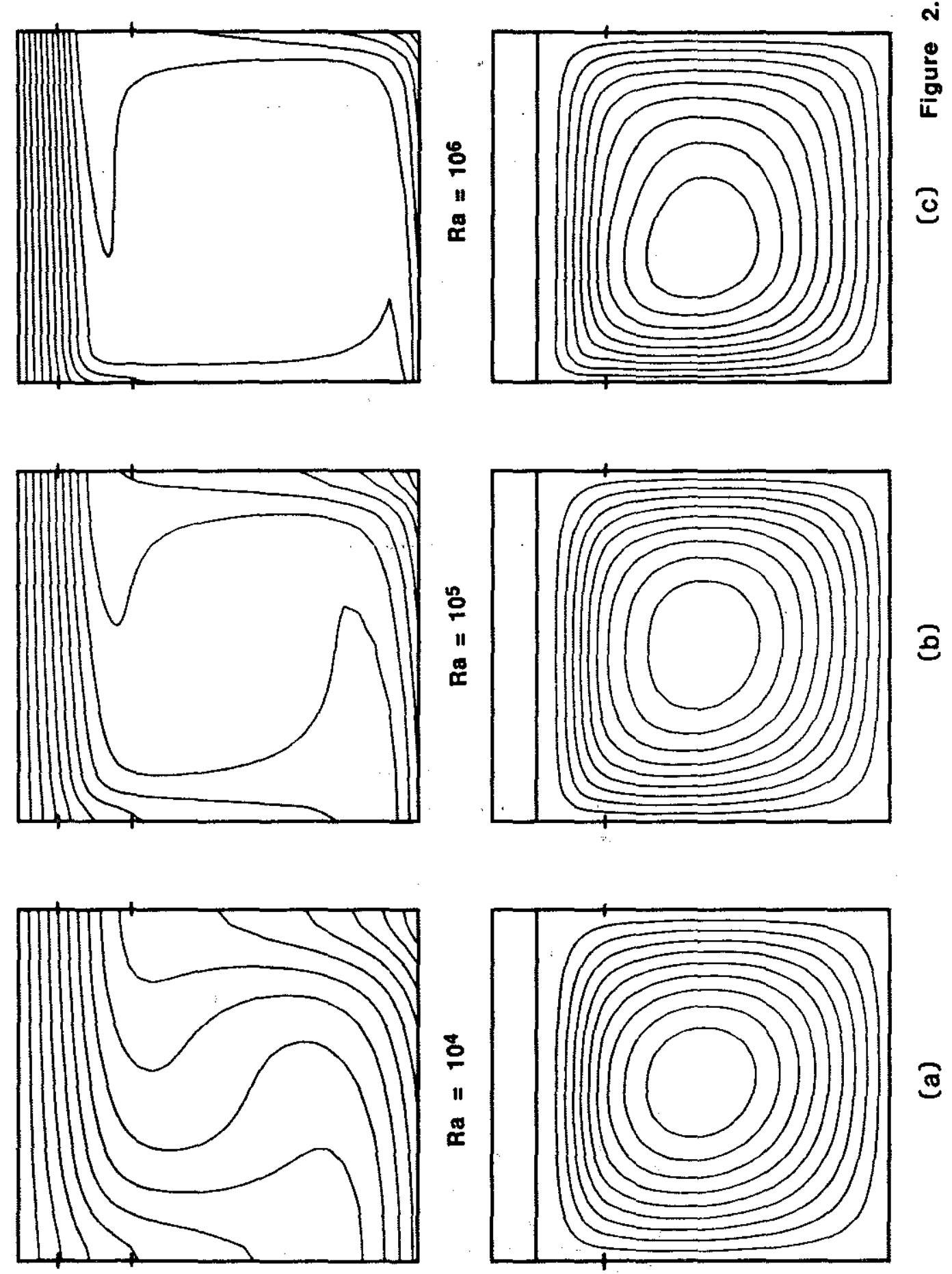


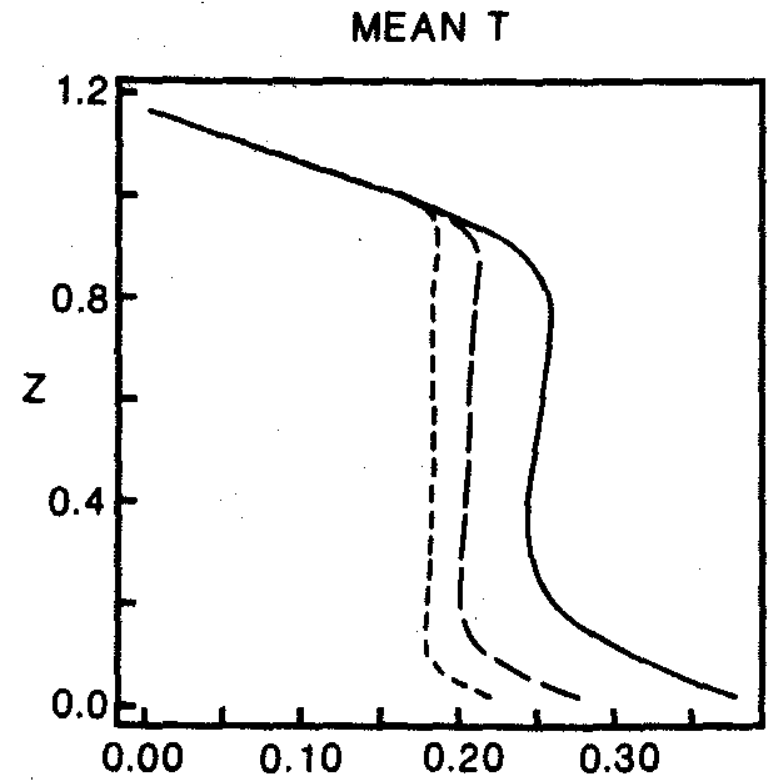

FIRST HARMONIC

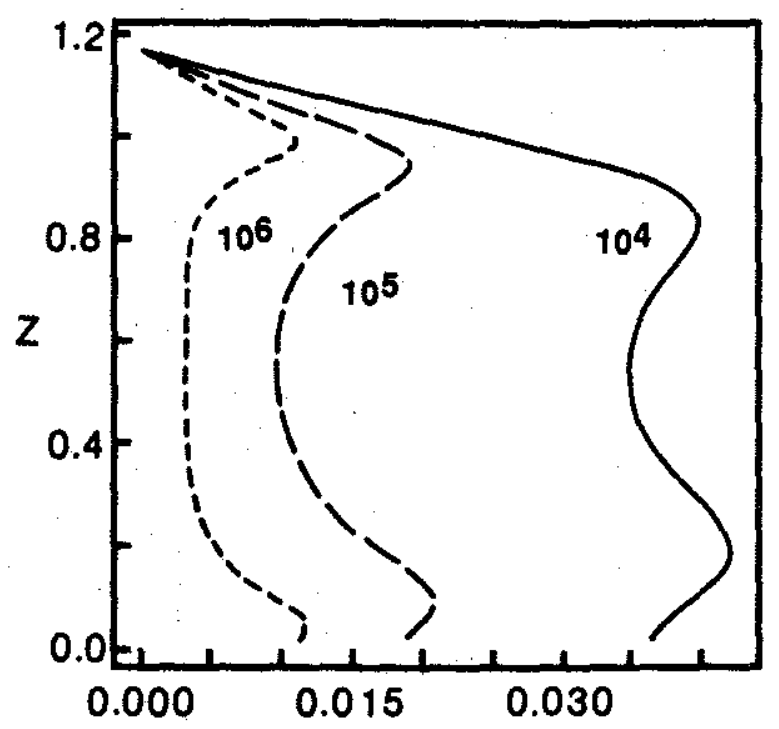

Figure 2.5 

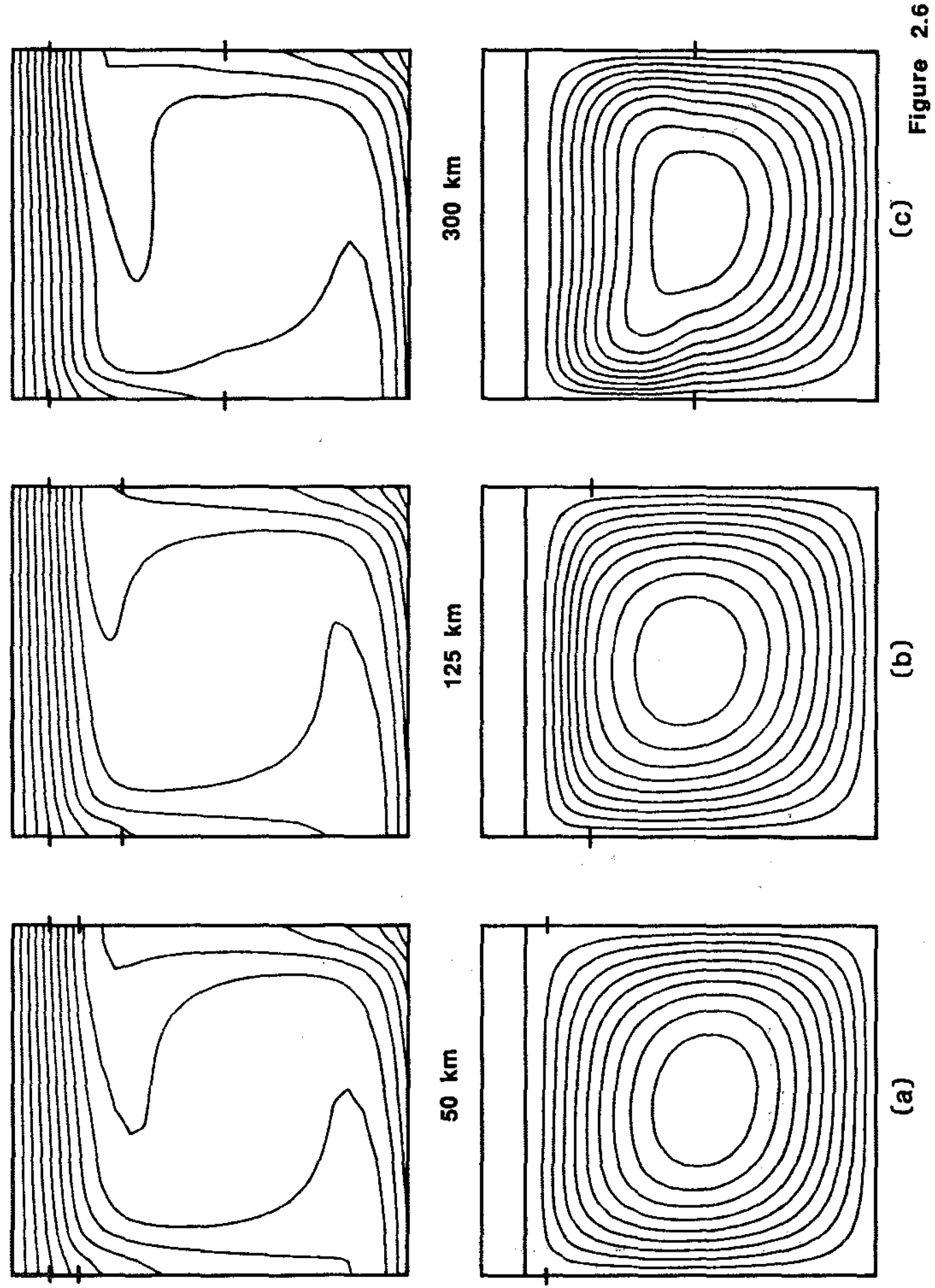


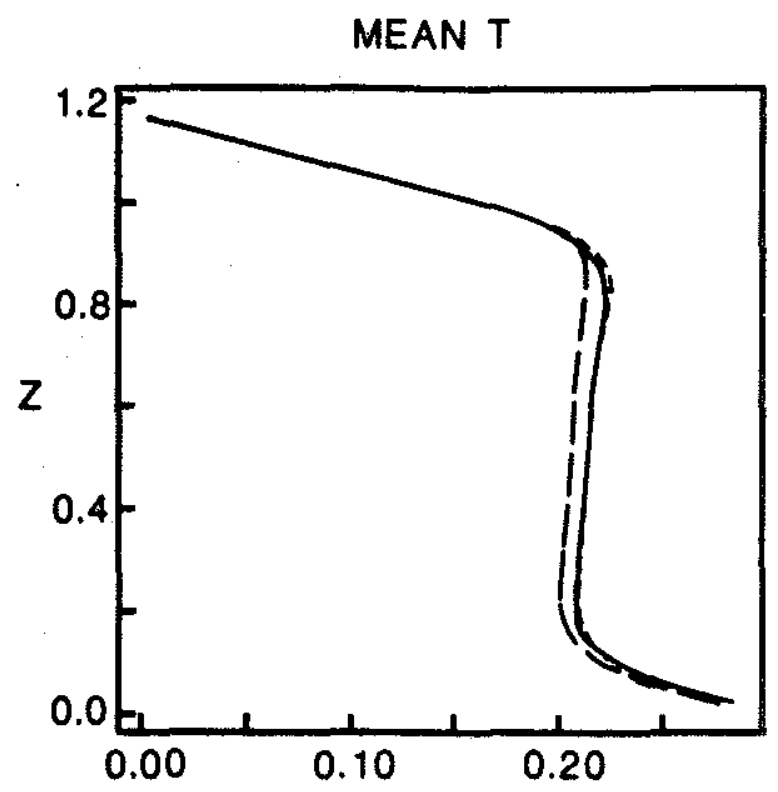

FIRST HARMONIC

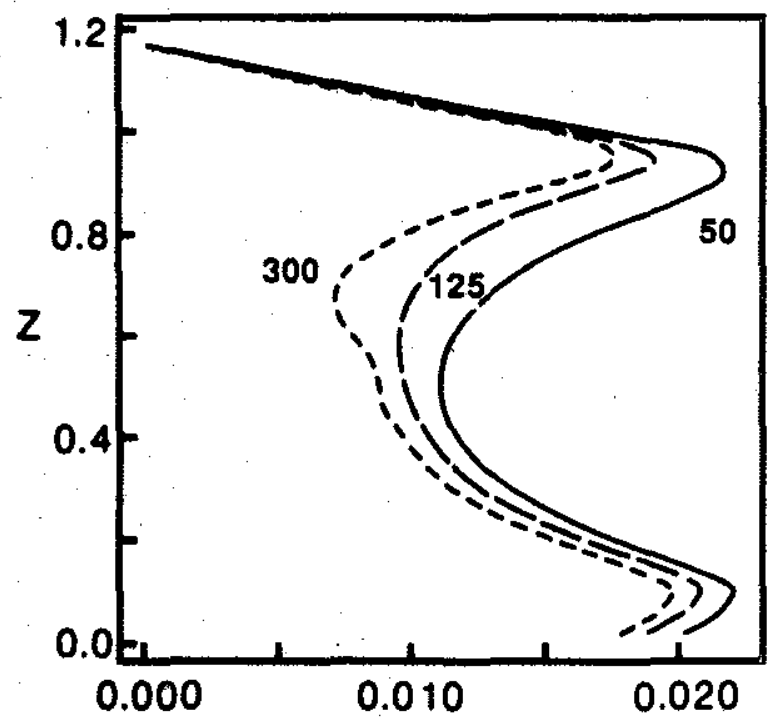

Figure 2.7 

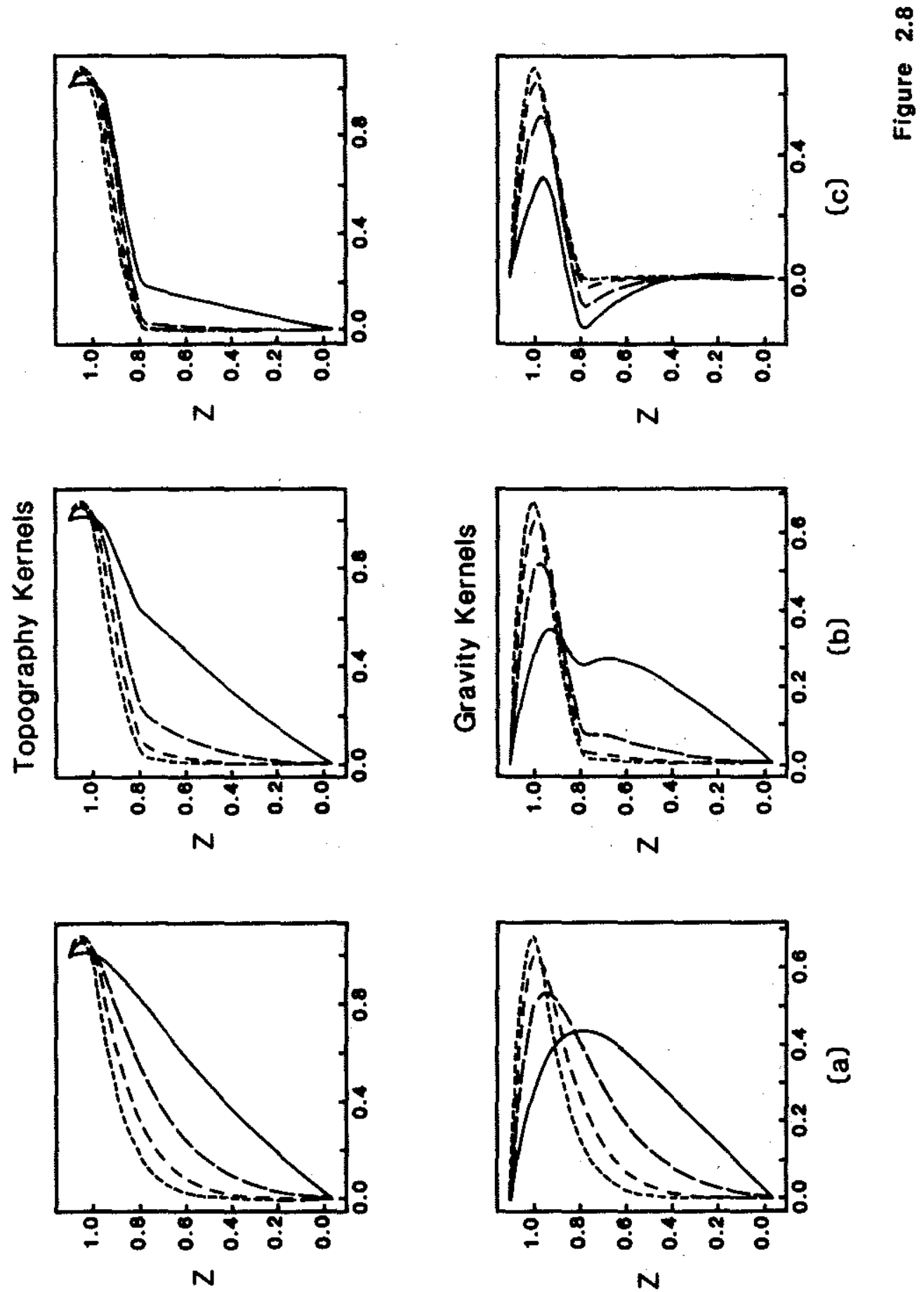

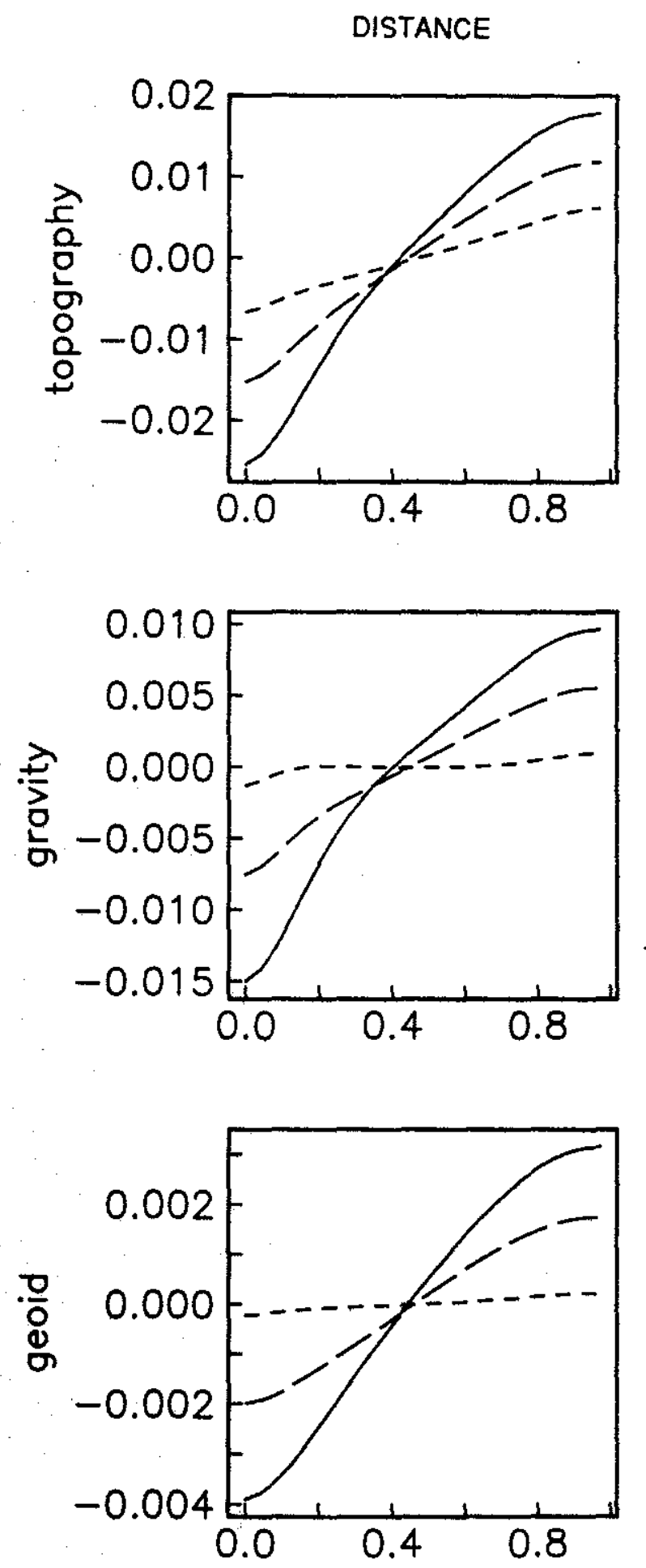

Figure 2.9 
운

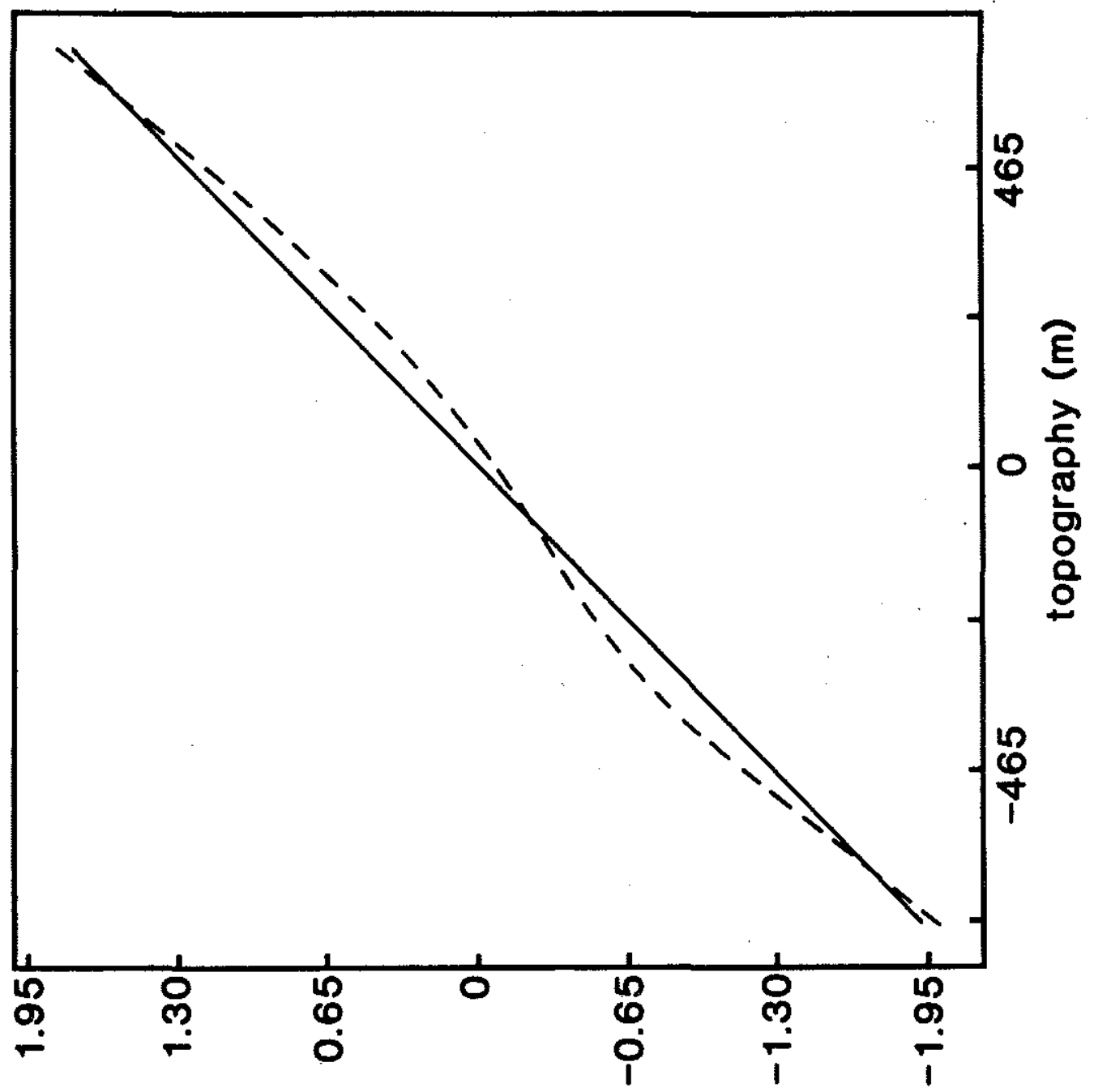

(w) p!̣oø6 

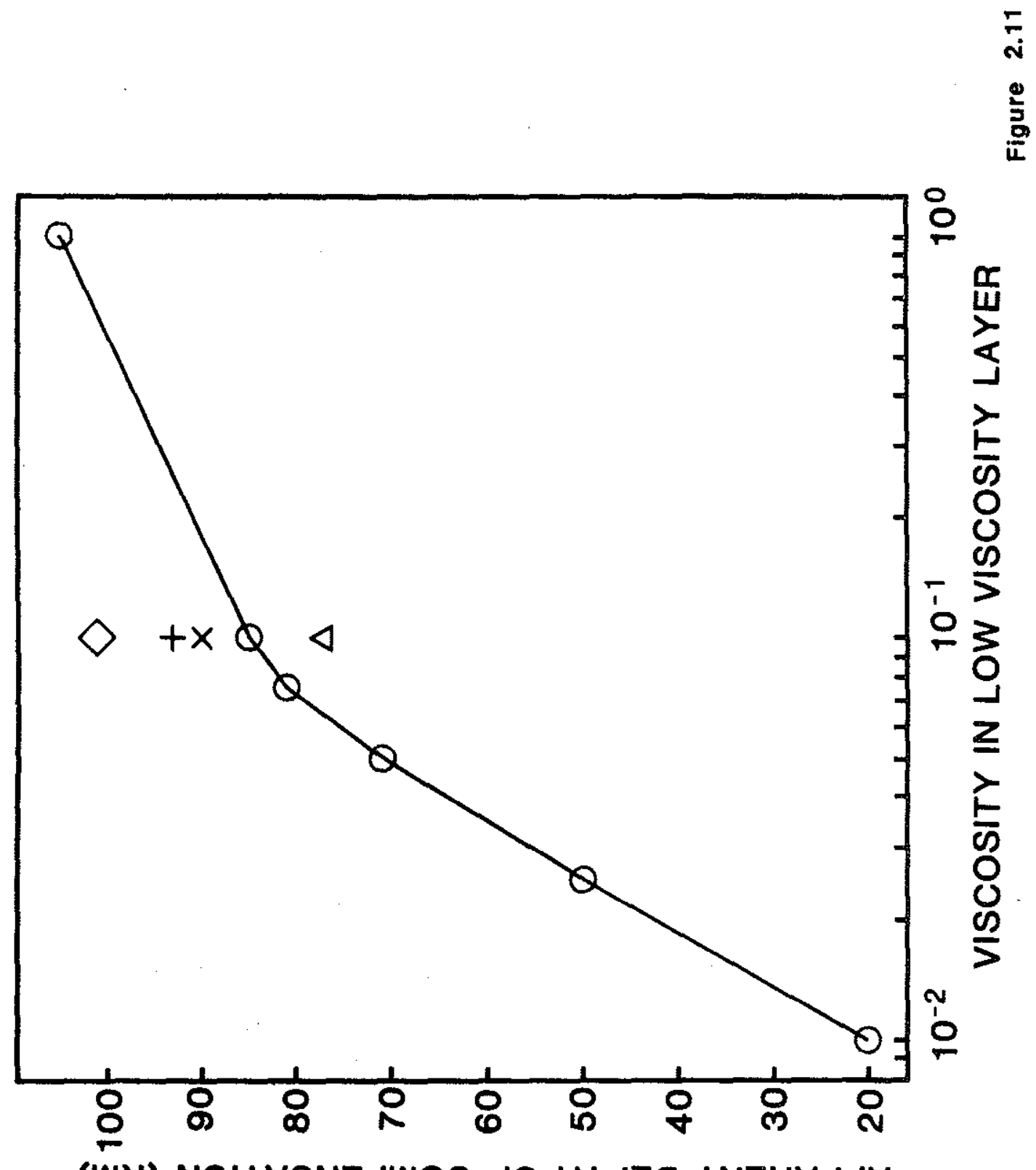

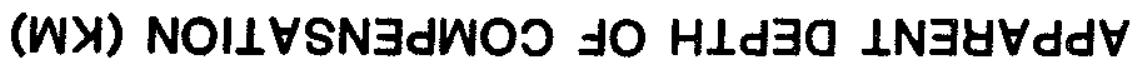



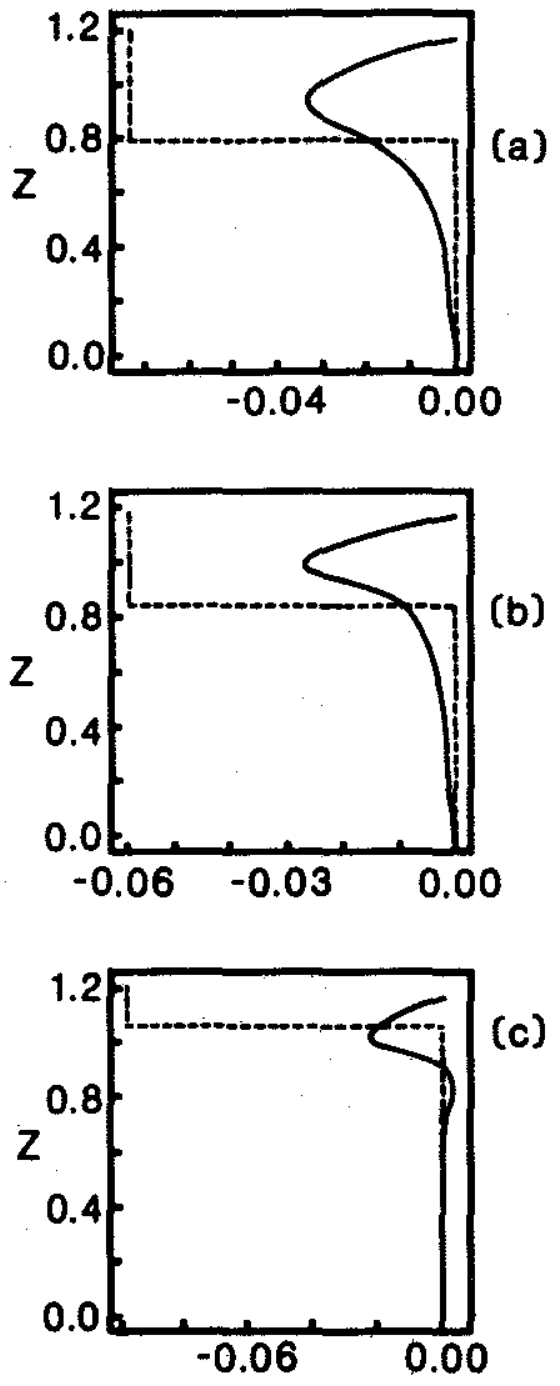

DISTANCE

Figure 2.12 


\section{Chapter 3: THE FORMATION OF MID-PLATE SWELLS}

\subsection{Introduction}

Mid-plate swells are regions of the seafloor associated with large long wavelength $(1000-2000 \mathrm{~km})$ topographic, geoid and heat flow highs (Betz and Hess, 1942; Dietz and Menard, 1953; Crough, 1978; Detrick and Crough, 1978; Von Herzen et al., 1982). Often they are crested by hotspot volcanism, creating such spectacular hotspot island chains as the Hawailan and Marquesas islands. Since mid-plate swells cannot be explained by thermal cooling of the plates or by lithospheric faulting or deformation, they must be associated with processes that originate in the mantle (Dietz and Menard, 1953; Crough, 1978; Detrick and Crough, 1978). In this paper, we present a convective model of their formation.

From detailed geophysical studies at a number of swells, we can draw some broad conclusions about mid-plate swells (Crough, 1978). First, swells typically rise to 1-2 $\mathrm{km}$ above the average depth for seafloor of similar age, with the Cape Verde Rise having the largest amplitude at over 2 $\mathrm{km}$ in height (Crough, 1982; McNutt, 1987). The geoid and heat flow anomalies for most swells range between $6-13 \mathrm{~m}$ and. 6-16 $\mathrm{mw} / \mathrm{m}^{2}$, respectively, with Cape Verde again the largest with a geoid anomaly of nearly $13 \mathrm{~m}$ and a heat flow anomaly of $16 \mathrm{~mW} / \mathrm{m}^{2}$ (Detrick et al., 1981; Von Herzen et al., 1982; Detrick et al., 1986; Courtney and White, 1987; McNutt, 
1987). Second, the geoid and topography anomalies are in general linearly related, giving depths of compensation which lie between $45-70 \mathrm{~km}$, well within the thermal plate thickness (Crough, 1978; Haxby and Turcotte, 1978; Detrick et al., 1981; McNutt and Shure, 1986; Fischer et al., 1986): Finally, recent studies of the flexure at swells have shown that they can also be characterized by thin elastic plates, which are thinner than the thickness expected for lithosphere of equivalent age (Watts, 1978; McNutt and Shure, 1986).

The Hawaiian swell in the Pacific is perhaps the best studied swell to date and has provided insight into the formation of mid-plate swells. It appears to have been active over at least the last $60 \mathrm{~m} \cdot \mathrm{y}$. and to have created the Emperor and Hawaiian seamount chains, which are elongated in the direction of plate motion. It has a topography anomaly of $1.0-1.4 \mathrm{~km}$, a geoid anomaly of $6-10 \mathrm{~m}$ and a heat flow anomaly of $5-9 \mathrm{~mW} / \mathrm{m}^{2}$, along with an apparent depth of compensation of 60-80 km and an elastic plate thickness of 25-35 km (Watts, 1978; Detrick and Crough, 1978; Von Herzen et al., 1982; Haxby and Weissel, 1986; McNutt and Shure, 1986). Throughout the Emperor and Hawaiian seamount chains, the seamounts become progressively younger towards the islands of Hawaii and the newly forming Loihi (Jarrard and Clague, 1977). Under the youngest islands, the swell is at a maximum in both its topography and geoid anomalies. From the first upstream evidence of 
the swell to the location of these maximums, the plate ages by less than $10 \mathrm{~m} . \mathrm{y}$. (Detrick and Crough, 1978; McNutt and Shure, 1986). Downstream along the swell, the topography and geoid anomalies subside in a manner similar to the thermal subsidence of normal seafloor of effectively younger age (Detrick and Crough, 1978).

From the general characteristics of swells and from the case of the Hawailan swell, we can place some constraints on their formation. Because swells exhibit high heat flow anomalies and are often crested by hotspot volcanism, their uplift is probably related to a thermal source (Detrick et al., 1981; Von Herzen et al., 1982; Detrick et al., 1986). This thermal source is capable of maintaining the uplift observed at swells for periods greater than $60 \mathrm{~m} . \mathrm{y} .$, such as at the Hawailan swell, and often without continuous volcanism at the surface, as in the case of Bermuda and cape verde. Furthermore, since the relative motion between hotspots is much less than that between the surface plates, they form a reasonably consistent reference frame over times much longer than the creation and destruction of the plates (Molnar and Atwater, 1973). When distant from this source, the swell subsides in accordance with conductive cooling and, from the depth and geoid-age data at the Hawaiian swell, it also appears that the major uplift of the swell occurs within $10 \mathrm{~m} \cdot \mathrm{y}$. of passing over the hotspot source (Crough, 1978; Detrick and Crough, 1978; MCNutt and Shure, 1986). Because conductive cooling through the oceanic 
plates is characterized by a much longer time scale, such a short uplift time points to a dynamic mechanism for the uplift of swells.

Two classes of models have been proposed for the formation of mid-plate swells. The first class - conductive cooling models - assert that the isotherms at depth with in the lithosphere have been displaced upwards by tens of kilometers by an unspecified mechanism. This compression of the temperature structure to shallower depths produces depth, geoid and heat flow highs. Moreover, the depth of compensation becomes shallower. In one permutation of this model (called "plate thinning"), Crough (1978) and Detrick and Crough (1978) suggested that the plate acts rigidly down to depths corresponding to the thermal plate thickness, but underneath swells it has been thinned and the temperatures under a depth $d_{t}$ have been reset to $T_{m}$, the average temperature of the uppermost mantle. The material is then allowed to cool both above and below the depth $d_{t}$. These simple one parameter models produce a remarkable fit to the data at many swells, but not at all of them (McNutt, 1987). For example, the depth anomaly, elastic plate thickness and heat flow anomaly at the Hawaiian swell can be explained by thinning the $80 \mathrm{~m} \cdot \mathrm{y}$. old plate on which it resides to $40 \mathrm{~km}$ (Detrick and Crough, 1978; Menard and McNutt, 1982), but the Cape Verde rise cannot be explained by plate thinning (Courtney and White, 1986; McNutt, 1986). Moreover, the mechanism which emplaces these high 
temperatures at shallow depths within the short rise times measured at Hawaii is not apparent. In particular, Emerman and Turcotte (1983) showed that if the plate acts rigidly down to the thermal plate thickness, as in the original plate thinning models, the advective mechanisms cannot produce the required lithospheric thinning in the time allowed. Yuen and Fleitout (1985) demonstrated, however, that if the plate does not act rigidly down to these depths, but has a viscosity which depends on temperature and pressure, then convective flow may be sufficient to thin the plate.

The second class of models are convective or "plume" models as proposed originally by Dietz and Menard (1953). In these models, swells are the surface expression of convection cells. In the upper portion of the thermal plate, the plate behaves rigidly, but below this rigid portion the plate can deform ductily and participate in the flow (Parsons and McKenzie, 1977; Parmentier, 1978). In particular, a plume can penetrate and replace the conductively cooled layer with hot upwelling material. When the viscosity is constant throughout the mantle beneath the conducting lid, a convective model can produce the general shape of the swells but not their shallow depths of compensation or their elastic plate thicknesses, with the possible exception of the very large amplitude Cape Verde swell (Courtney and White, 1986). 
However, the viscosity of the upper mantle is not thought to be constant and, in particular, the uppermost mantle is most likely characterized by low viscosities. Much evidence suggests that a low viscosity zone exists beneath the surface plates from (1) theoretical calculations of the viscosity structure with depth in the oceanic mantle (Turcotte and Oxburgh, 1967; Parmentier, 1978; Fleitout and Yuen, 1984; Buck and Parmentier, 1986), (2) experimental results on the presence of melt in olivine and studies of melt production in the upper mantle (Cooper and Kohlstedt, 1984; McKenzie, 1984), (3) seismic studies of the uppermost oceanic mantle (Anderson and Sammis, 1970; Solomon, 1972; Eorsyth, 1977; Weilandt and Knopoff, 1982), and (4) studies of the evolution of the thermal structure at fracture zones (Craig and McKenzie, 1986; Robinson et al., 1987b, see Chapter 4). Robinson et al. (1987a; Chapter 2) showed that apparently shallow depths of compensation can result from convective processes in the mantle when a low viscosity zone exists in the uppermost mantle. Therefore, shallow depths of compensation cannot be used to argue against the dynamic uplift of mid-plate swells.

In this paper, we extend the results of Robinson et al. (1987a; Chapter 2) to a complete study of the effect of the low viscosity zone on the formation of mid-plate swells. To isolate the effect of a low viscosity zone on mantle flow and its surface observables, we have simplified the viscosity structure in our model to a three layered 
structure, consisting of a conducting lid overlying a low viscosity layer which in turn overlies a constant viscosity layer extending to the base of the upper mantle. We have ignored, however, the effects of temperature and pressure on the viscosity and the changes in the viscosity structure with time resulting from its temperature dependence. Instead, we vary the Rayleigh number, layer thicknesses and viscosity contrast in the model to approximate the range of possible viscosity structures.

The paper is divided into three parts. First, we calculate the convective temperature and velocity solutions for different combinations of the viscosity in the top layer, the fluid layer thicknesses and the Rayleigh number based on the viscosity in the bottom layer. Second, from these temperature solutions, we calculate the geoid, topography and heat flow anomalies, the elastic plate thickness, the depth of compensation and an upper bound on the uplift time that result from the flow. Finally, we compare the results of our calculations to data at the Hawail, Bermuda, Cape Verde and Marquesas swells.

We have found that we can explain the anomalies observed at these four mid-plate swells with reasonable values for the Rayleigh number, layer thickness and viscosity contrast, taking into account the thickening of the lithosphere with age. We cannot constrain the thickness of the low viscosity zone from the data at swells. Rather, its depth is constrained by seismic observations of a low 
velocity zone (e.g. Weilandt and Knopoff, 1982) and by the wavelength of the small scale instabilities in the cooling oceanic plates (Haxby and Weissel, 1986; Robinson and Parsons, 1987; Chapter 5). Both of these observations give estimates for the thickness of the low viscosity zone which range from 100-200 km. Since the Rayleigh number and viscosity contrast have the same effect on the observables (but not to the same extent), we cannot predict the exact values of the parameters underneath the swells from the anomalies. However, the depth of compensation is very sensitive to the viscosity contrast, so that we can estimate the viscosity contrast and the best fitting Rayleigh number. The viscosity contrasts required underneath the swells range from 0.1-0.2 at Cape Verde Rise to 0.01-0.03 at the Marquesas swell at a Rayleigh number of $10^{6}$. Eurthermore, the viscosity contrasts that are required at each of the swells decreases as the age of the lithosphere on which the swell resides increases, which is consistent with theoretical predictions of the viscosity from the temperature and pressure conditions thought to exist in the upper mantle (Fleitout and Yuen, 1984; Buck and Parmentier, 1986).

\subsection{The Numerical Model of the Flow}

We use a finite element numerical mesh with three horizontal layers to model the flow (Figure 3.1). The uppermost layer is a rigid conducting lid. Beneath the lid 
is a low viscosity layer, and underlying the low viscosity layer is a constant viscosity layer which extends to the base of the upper mantle. The two fluid layers together have an aspect ratio of one. We impose a constant heat flux through the bottom boundary and hold the temperature at zero on the top of the conducting lid which initiates convection throughout the two fluid layers. Both side walls are stress-free and have reflective boundary conditions on the temperature. The conducting lid is rigid and the bottom boundary is stress-free. We have nondimensionalized the governing equations with the following transformations:

$$
\begin{aligned}
\mu^{\prime} & =\mu_{\circ} \mu \\
\left(x^{\prime}, z^{\prime}\right) & =d(x, z) \\
T^{\prime} & =\Delta T T \\
t^{\prime} & =\left(d^{2} / \kappa\right) t \\
\rho^{\prime} & =\rho_{O} \rho
\end{aligned}
$$

where primes denote dimensional quantities and $\mu_{0}$ is the kinematic viscosity of the bottom constant viscosity layer, $x$ and $z$ are the horizontal and vertical coordinates respectively, $d$ is the depth of the convecting layer, $k$ is the thermal diffusivity, $\rho_{\circ}$ is the reference density and $\Delta \mathrm{T}$ is the reference temperature given by the constant flux condition:

$$
\Delta \mathrm{T}=\mathrm{Fd} / \mathrm{k} \rho_{\circ} \mathrm{C}_{\mathrm{p}}
$$

where $F$ is the prescribed flux and $C_{p}$ the specific heat. We used the values for these parameters as given in Table 3.1. 
The equations of motion, heat transport and state are then given by (omitting the primes):

$$
\begin{gathered}
\nabla \cdot \sigma=-R\left(T-T_{0}\right) \hat{z} \\
\partial \mathrm{T} / \partial t+u \cdot \nabla T=\nabla^{2} \mathrm{~T} \\
1-\alpha \Delta \mathrm{T}\left(\mathrm{T}-\mathrm{T}_{0}\right)=\rho
\end{gathered}
$$

where $u$ is the velocity vector, $\alpha$ is the thermal expansion coefficient, $\hat{z}$ is a vertical unit vector, $\sigma$ is the stress tensor given by:

$$
\sigma_{i j}=-p \delta_{i j}+\mu\left(\partial u_{i} / \partial x_{j}+\partial u_{j} / \partial x_{i}\right)
$$

and $R$ is the Rayleigh number:

$$
R=g_{0} \alpha \Delta \mathrm{Td}^{3} / \mu_{0} \kappa
$$

where $g_{0}$ is the acceleration of gravity. These equations are solved using a velocity based finite element method (Daly and Raefsky, 1985). Although the incompressibility equation:

$$
\nabla \cdot u=0
$$

is never explicitly solved, a penalty function treatment of the pressure (Hughes et al., 1979) ensures

incompressibility. In the conducting lid the velocities, set to zero, mimic infinite viscosity (Jaupart and Parsons, 1985). The boundary conditions are summarized in Figure 3.1 .

All of the convection calculations were run to steady state with the implicit time stepping method described by Brooks (1981). To resolve the boundary layer flow, we often used a nonuniform grid with double or quadruple the number 
of grid points in the vertical direction in the low viscosity zone.

We first checked the resolution and steadiness of this flow by comparing the average flux at different depths to their steady state values. Due to the complex interaction of the boundary layers with the low viscosity zone, we also checked, for convergence, the runs on uniform grids with an explicit finite difference time stepping routine (Brooks, 1981) for a convective overturn time. To ensure that we had also resolved the flow, however, we ran a number of the calculations on very large uniform grids where we doubled, and in some cases tripled, the number of nodes in both directions and compared the results of these runs to those on the smaller grids.

In Table 3.2, we have listed the parameters for all of the runs in this parameter study. We have primarily varied the relative viscosity in the two layers, the Rayleigh number and the relative thicknesses of the layers. However, we have also chosen different boundary conditions for the bottom boundary, and have compared calculations with and without the conducting lid. We ran most of our models with a conducting lid thickness of $0.125(75 \mathrm{~km})$ and a top layer thickness of $0.21(125 \mathrm{~km})$, encompassing the top $200 \mathrm{~km}$ of the upper mantle. We chose these parameters to agree with the seismic evidence and small scale convection anomalies which constrain the low viscosity zone to near a $125 \mathrm{~km}$ thickness (Weilandt and Knopoff, 1982; Robinson and Parsons, 
1987), and to agree with the melting models of the upper mantle (e.g. Mckenzie, 1984) which place the region of high mantle melt within the top $200 \mathrm{~km}$. The conducting lid corresponds to the mechanical portion of a $80 \mathrm{~m} \cdot \mathrm{y}$. lor older) plate.

\subsection{The Convective Flow}

To approximate the convective flow at swells, we have run our calculations to steady state where possible. In Figure 3.2, we show the temperature solutions for a Rayleigh number of $10^{5}$ and a top layer thickness of $125 \mathrm{~km}$, as we vary the viscosity contrast from 1.0 to 0.001 . In Figure 3.3, we have drawn the steady state temperature solutions at a viscosity contrast of 0.01 and a top layer thickness of $125 \mathrm{~km}$ as we vary the Rayleigh number from $10^{4}$ to $10^{6}$. In Figure 3.4(a) and 3.4(b), we have drawn the horizontally averaged temperature structure for the solutions shown in Figures 3.2 and 3.3 , respectively. As the low viscosity decreases and as the Rayleigh number increases, the mean temperature in the interior of the cell decreases (if $\Delta \mathrm{T}$ is held constant). Furthermore, as we increase the effective Rayleigh number in the top layer, either by increasing the overall Rayleigh number or decreasing the viscosity in the layer, the top boundary layer thins. Where the viscosity jumps across the boundary between the layers, the upwelling and downwelling plumes are pinched and expanded, 
respectively, to accommodate the change in the boundary layer thickness.

In Figure 3.5, we have plotted the top boundary layer thickness versus the viscosity contrast for a number of Rayleigh numbers. We defined the boundary layer thickness in the same manner as Jaupart and Parsons (1985), i.e. the depth at which the boundary layer temperature equals the interior temperature when the boundary layer temperatures are extrapolated linearly downward with a slope equal to the mean surface gradient. As expected, when the Rayleigh number increases the boundary layer thickness decreases. What is perhaps more interesting is that an order of magnitude decrease in the viscosity of the low viscosity layer produces a thinner boundary layer than an order of magnitude increase in the Rayleigh number keeping the viscosity contrast constant. However, the effect of an order of magnitude increase in the viscosity contrast would be equivalent to an order of magnitude increase in the Rayleigh number if only the change in the Rayleigh number of the boundary layer were considered (McKenzie et al., 1974). The extra boundary layer thinning occurs in response to an apparent change in the top boundary condition. The base of the conducting lid is rigid, but the presence of a low viscosity layer next to it reduces the transmission of stress at the boundary as compared with, the constant viscosity case. Also, due to the change in the effective Rayleigh number of the boundary layer as discussed above, 
the velocities in the boundary layer immediately next to the rigid boundary increase and the boundary layer thins.

However, these are also the effects of a no-slip boundary if the viscosity in the layer were not reduced. The boundary appears, therefore, like a no-slip boundary to the flow in the bottom layer, causing the extra boundary layer thinning observed in the calculations. Furthermore, when the effective Rayleigh number in the top boundary layer increases above its critical Rayleigh number, the boundary layer can become unstable as in Figure 3.2 and 3.4. This instability begins downstream of the upwelling plume with a wavelength of close to $200 \mathrm{~km}$ before it is swept downstream into the downwelling.

In Figure 3.6 , we have plotted the average temperature of the convecting cell and the conducting lid for all of the calculations with a low viscosity zone that is $0.21(125 \mathrm{~km})$ thick. As the viscosity contrast increases, the average temperature decreases and, at a viscosity contrast of 0.001 , the average temperature decreases to half of its value with no viscosity contrast. Together the boundary layer thickness and the average temperature provide a useful characterization of the flow (McKenzie et al., 1974).

In Figure 3.7, we present the temperature solutions as we vary the thickness of the low viscosity layer. We have kept the viscosity contrast constant at 0.1 and the Rayleigh number at $10^{5}$. In Figure $3.4(c)$, we have drawn the mean 
temperature structure with depth for the runs pictured in Figure 3.7 and run 29 where the low viscosity zone fills half of the box. At the intermediate layer thickness of $0.21(125 \mathrm{~km})$, the mean temperature in the adiabatic portion of the cell is the least of the four runs. This behavior in the mean temperature as a function of the layer thickness reflects the effect of the apparent boundary condition as discussed above. At a layer thickness which is slightly greater than the top boundary layer thickness, the low viscosity layer can effectively alter the velocity gradient in just the top boundary layer so that the flow in the bottom layer responds as if the top boundary conditions were no-slip conditions. If the low viscosity layer is much thinner than the top boundary layer, however, it cannot effectively thin the top boundary layer. Also if the low viscosity layer is much thicker than the top boundary layer, it alters the thickness and temperature drop across the side boundary layers as well, so that the flow in the bottom layer responds as if the Rayleigh number of the fluid layers was increasing. Therefore, a low viscosity zone with a thickness comparable to the top boundary layer thickness will be most effective at decreasing the boundary layer thickness and the temperature drop across it.

\subsection{Calculation of the Surface Expression of the Flow}

At the Hawaiian, Bermuda, Cape Verde and Marquesas swells, we can measure the peak-to-peak amplitudes of the 
topography and geoid anomalies. Estimates also exist for their depths of compensation and elastic plate thicknesses. only at Hawaii, however, do we have an estimate of the uplift time and we do not have reliable heat flow measurements at the Marquesas. In this section, we will describe how we generate theoretical uplift, geoid, heat flow, and elastic plate thickness from the temperature structure derived from a convective model.

\subsubsection{Topography, Gravity and Geoid}

To calculate the topography and geoid anomalies, we use the Green's function method developed by Parsons and Daly (1983). Robinson et al. (1987a; Chapter 2) have discussed in detail the application of this method to a three layered viscosity model. Briefly, however, it decomposes the temperature field into its Fourier components in the horizontal direction. At each wavenumber, k, the Green's function response of the geoid and topography to the temperature anomalies with depth, the gravity and topography kernels, are calculated. The Fourier components of the temperature are then integrated with depth weighted by the appropriate kernel, and then Fourier transformed back to produce the surface anomaly.

The low viscosity zone strongly affects the response of the topography, gravity and geoid fields to the temperature structure at depth. Since the low viscosity zone decreases the transmission of normal stress through the low viscosity layer, it diminishes the effect of the temperature 
anomalies, in and below the low viscosity zone, on the surface topography. The gravity anomaly, however, represents the sum of the gravitational contribution from the topography on the boundaries and the density variation within the layer. Because the low viscosity zone decreases the response of the surface topography to the temperature anomalies in and below the low viscosity zone, the internal density distribution dominates the gravitational contribution from a depth inside or below the low viscosity zone. Therefore, the positive temperature anomalies in this region counteract the positive effects from shallower temperature variations.

\subsubsection{Heat Elow}

The heat flow reflects the near surface temperature gradients and is given by:

$$
\mathrm{q}^{\prime}=\mathrm{k}_{\mathrm{C}} \mathrm{d \textrm {T } ^ { \prime }} / \mathrm{dz^{ \prime }}
$$

where $k_{c}$ is the thermal conductivity. To calculate $\mathrm{dT} / \mathrm{dz}$, we take the vertical derivative of the temperature between the top two grid points of the finite element mesh.

\section{4 .3 Depth of Compensation}

The depth of compensation is usually defined as the depth under which there does not exist a horizontal pressure gradient due to the source of the topography. Since convective flow extends to large depths in the mantle, this. definition does not apply directly to a convective mechanism for the uplift of the swell. Nevertheless, we can calculate an "apparent depth of compensation" for our models assuming 
Pratt compensation (which is often used to calculate the depth of compensation of mid-plate swells). With Pratt compensation, the topography is supported by horizontal density variations above a depth $2 d_{c}$, where $d_{c}$ is the equivalent Airy compensation depth (Haxby and Turcotte, 1978; Parsons and Daly, 1983). Below the compensation depth the pressure due to the topography is zero. The geoid anomaly due to a topography anomaly of wavenumber, $k$, which is compensated by a horizontal variation in density above a depth $2 d_{C}$ is then given by:

$$
N^{\prime}(k)=\left[2 \pi G\left(\rho_{O}-\rho_{W}\right) d_{C} / g_{O}\right] h^{\prime}(k \cdot)
$$

at wavelengths large compared with $d_{C}$. Plots of topography versus the geoid over a swell would then have a slope proportional to $d_{C}$. We estimate this slope as outlined in Robinson et al. (1987a; Chapter 2).

\subsubsection{Elastic Plate Thickness}

In "normal" oceanic lithosphere, the elastic plate thickness is roughly proportional to the square root of the age of the lithosphere when loaded (Watts, 1978). Because the elastic properties of the plates depend on temperature and because the isotherms in a conductively cooling plate deepen as a function of the square root of age, this result is often interpreted to indicate that the elastic plate thickness is controlled by an isotherm (Watts, 1978; McNutt and Menard, 1982). Estimates for the temperature represented by this isotherm vary from $450^{\circ} \mathrm{C}$ to $600^{\circ} \mathrm{C}$ (Watts, 1978; McNutt and Menard, 1982). Therefore, we 
estimate a range of values for the elastic plate thickness from the depths to the $450{ }^{\circ} \mathrm{C}-600{ }^{\circ} \mathrm{C}$ isotherms underneath the apex of the swell.

\section{4 .5 Uplift Time}

Since we cannot directly model the onset of a swell with the results of steady state calculations, we performed a time dependent calculation in which we solved the equations of motion and incompressibility on a finite element grid and the temperature equation on a finite difference grid. We first replaced the temperatures at the grid points in the top layer in our steady state solutions with their horizontally averaged mean values. We then let the flow progress and re-establish the convective cell. We defined the "replenishment time" as the time required for the peak-to-peak amplitudes of the topography and geoid anomalies to reach $95 \%$ of their steady state values. Because the flow is three dimensional and because this model represents the extreme case of the emplacement of an isolated plume, this estimate of the uplift time can only be an upper bound. Still it gives a good indication of the effect of the low viscosity zone on an upwelling plume as it encounters a conducting boundary layer.

In Figure 3.8, we plot the peak-to-peak amplitude of the topography anomaly through such a calculation at a Rayleigh number of $10^{5}$, a viscosity contrast of 0.1 and a layer thickness of $0.21(125 \mathrm{~km})$. The peak-to-peak anomaly initially rises and overshoots the steady state value of 
$1.25 \mathrm{~km}$ but, after $100 \mathrm{~m} . \mathrm{y} .$, it begins to decrease to the steady state value. The initial overshoot of the steady state value is due to the localized effects of the upwelling and downwelling plumes on the initial horizontally averaged temperature structure. As the cell reaches steady state the upwelling and downwelling have spread out in the low viscosity zone and the temperature differences are less localized, so that the resultant topography is smaller. As the viscosity contrast increases and the plumes can spread into the top layer more quickly, the amount that the peakto-peak topography anomalies overshoot their steady state values decreases and, by a viscosity contrast of 0.01 , the peak-to-peak values do not overshoot their steady state values, but rise smoothly to them.

\subsection{The surface Expression of the Flow}

Since it is difficult to obtain a profile over a swell. in which the topography and geoid anomalies result from only the convective temperature anomalies at depth, we do not compare our results to the anomalies across the swell, but predict their peak-to-peak amplitudes. Furthermore, since the scaling factors for the topography geoid and heat flow anomalies depend on the constants, $\alpha$ and $\kappa$, which are not well known, we present in this section only nondimensional values for the anomalies.

In Figures $3.9(a)$ and $3.9(b)$, we have drawn the nondimensional geoid and topography anomalies for run $1 \mathrm{~b}$ 
(from Table 3.2). In general these anomalies are dominated by the longest wavelength in the box - twice the depth of the fluid layer - and are elevated above upwelling and depressed above downwellings. Swell anomalies are also dominated by their longest wavelengths corresponding to their full widths of $1000-2000 \mathrm{~km}$ (Crough, 1978). In Figures 3.10 and 3.11 , we have plotted the estimated peakto-peak amplitudes of the geoid and topography anomalies, respectively, versus the viscosity of the top layer for all of the runs with a top layer thickness of $125 \mathrm{~km}$ and Rayleigh numbers of $10^{5}, 3.2 \times 10^{5}$ or $10^{6}$ (Table 3.2). As expected, the magnitude of the geoid and topography anomalies decrease with increasing Rayleigh number. Also, as the viscosity in the top layer decreases, the amplitudes of the geoid and topography anomalies decrease. In particular, for both the geoid and topography anomaly, an order of magnitude decrease in the viscosity of the top layer produces a greater reduction in the peak-to peak amplitude of the anomaly than an order of magnitude decrease in the Rayleigh number. This behavior primarily reflects the effect of the viscosity structure on the kernels. Below a viscosity of 0.075 in the top layer for a layer thickness of $0.21(125 \mathrm{~km})$, the geoid kernels are negative at depth, so that the temperature anomalies below this depth counteract the geoid effect of the anomalies above it. The topography kernels, on the other hand, remain positive everywhere. At viscosity contrasts greater than this 
critical viscosity contrast, therefore, the geoid anomalies decrease much faster than the topography anomalies.

In Figure 3.9(c), we present the nondimensional heat flow anomaly for run $1 b$ (from Table 3.2). In general, as for the geoid and topography anomalies, the heat flow anomaly is dominated by the longest wavelength in the box (twice the fluid layer depth) and is elevated above the upwellings while depressed above the downwellings. Because the heat flow anomaly results from conduction through the Iid, however, it is much smoother than the geoid and topography anomalies. In Figure 3.12, we have plotted the peak-to-peak amplitudes of the heat flow anomalies for all of the runs in Table 3.2 versus the viscosity in the top layer. Because the heat flow anomaly is controlled primarily by the temperature contrast across the top boundary layer, the amplitude of the heat flow anomaly decreases both with an increase in Rayleigh number (if $\Delta \mathrm{T}$ is held constant) and an increase in viscosity contrast.

In Figure 3.13, we have plotted the dimensional apparent depth of compensation in kilometers versus the viscosity in the top layer for runs in Table 3.2 where the top layer thickness is $125 \mathrm{~km}$ and the Rayleigh number is $10^{4}, 10^{5}$ or $10^{6}$. A detailed discussion of the behavior of the depth of compensation is presented in Robinson et al. (1987a). In general, the depth of compensation depends weakly on the Rayleigh number of the fluid; an order of magnitude increase in the overall Rayleigh number causes the 
depth of compensation to decrease by nearly $6 \mathrm{~km}$. The depth of compensation depends much more strongly on the viscosity structure of the top layer. In particular, for viscosity contrasts less than 0.075 and for a layer thickness of 0.21 $(125 \mathrm{~km})$, an order of magnitude increase in the viscosity contrast will produce a $15-20 \mathrm{~km}$ decrease in the depth of compensation, but for greater viscosity contrasts, the depth of compensation decreases much more rapidly. For example, at a Rayleigh number of $10^{5}$ and a layer thickness of 0.21 $(125 \mathrm{~km})$, the depth of compensation at a viscosity contrast of 0.1 is close to $80 \mathrm{~km}$, but with a viscosity contrast of 0.01 the depth of compensation has decreased to $20 \mathrm{~km}$. Since the depth of compensation is essentially the ratio of the peak-to-peak magnitudes of the geoid and topography anomalies, it is very sensitive to their behavior. This rapid decrease in the depth of compensation can, therefore, be explained by the rapid decrease of the geoid anomaly in comparison to the topography anomaly as discussed in Robinson et al. (1987a; Chapter 2).

For all of our runs with a conducting lid thickness of $75 \mathrm{~km}$, the elastic plate thickness lies between $24-31 \mathrm{~km}$ using the $450^{\circ} \mathrm{C}$ isotherm for the base of the elastic plate and $28-36 \mathrm{~km}$ using the $600^{\circ} \mathrm{C}$. These values are very similar to the elastic plate thicknesses found at most midplate swells (see Table 3.3) (Watts, 1978; McNutt and shure, 1986; Fischer et al., 1986; McNutt, 1987). For runs where we have varied the lid thickness (numbers 30-32 in Table 
3.2), we see a change in elastic plate thickness. For example, with a conducting lid thickness of $50 \mathrm{~km}$, the elastic plate thicknesses reduce to $17-23 \mathrm{~km}$ using the 450 ${ }^{\circ} \mathrm{C}$ isotherm and $21-27 \mathrm{~km}$ using the $600{ }^{\circ} \mathrm{C}$ isotherm.

In Figure 3.14, we have plotted the replenishment times in militions of years for all of the runs in Table 3.2, with a top layer thickness of $125 \mathrm{~km}$ and Rayleigh numbers of $10^{4}$, $10^{5}$ and $10^{6}$. The replenishment time decreases with an increase in the Rayleigh number and with a decrease in the viscosity of the top layer. At a Rayleigh number of $10^{6}$ and a viscosity contrast of 0.01 , the replenishment time decreases to $20 \mathrm{~m} . \mathrm{y}$.. This simple model cannot, therefore, explain the uplift times, but shows how the time scales decrease rapidly with Rayleigh number and, in particular, viscosity contrast. Furthermore, as discussed in the previous sections, the uplift time obtained by this method is an upper bound on the uplift times expected from convective mechanisms in the Earth.

\subsection{Comparison to the Data}

The theoretical results apply to the peak-to-peak amplitudes of the topography and geoid anomalies over swells. Since, at many swells, the topography and geoid anomalies are not estimated as peak-to-peak values, we have re-examined plots of the topography and geoid around the swells and estimated peak-to-peak values. Since heat flow anomalies are usually estimated as peak-to-peak amplitudes 
(Von Herzen et al., 1982; Detrick et al., 1986; and Courtney and White, 1987), however, we can take these values directly. Given the sparse coverage over the swell, it is very likely that the measurements miss the heat flow peaks, so that these values often underestimate the true heat flow anomaly (Von Herzen et al., 1982). Since the depth of compensation is estimated from the ratio of the topography and geoid anomalies, the estimate of the depth of compensation is fairly robust, as are the estimates of the elastic plate thicknesses and the estimate of the rise time at Hawaii. These values are summarized in Table 3.3.

There are also two points which we must consider when we apply the results of our model to data. First, and perhaps most important, is that we must choose appropriate values for the scaling constants, $\alpha$ and $\kappa$. These "constants" vary throughout the mantle. In particular, the thermal expansion coefficient is thought to vary with depth with higher values near the surface and lesser values at depth. The variation of $\boldsymbol{k}$ is not well known. In our calculations, since the kernels sample the upper portions of the mantle to a greater extent, we should choose values for these parameters between the surface and deep mantle values. For consistency, we have chosen values which are acceptable for the upper mantle (see Table 3.1).

Second, since we wish to illustrate that the observables are most sensitive to the viscosity contrast, we have kept the other variables held fixed at reasonable 
values and have varied only the viscosity in the low viscosity zone. From the convection and observable analyses presented earlier, we can extrapolate these results to different Rayleigh numbers, layer thicknesses and conducting lid thicknesses. However, we show that, by only varying the viscosity contrast and by taking into account the variation in lid thickness with age, we can explain the observables at the four swells.

We have chosen the fixed values for the Rayleigh number, layer thickness and conducting lid thickness from current estimates in the literature. First, estimates of the Rayleigh number in the upper mantle range from $10^{6}$ to $10^{7}$ and possibly to $10^{8}$ (McKenzie et al., 1974; Houseman and Mckenzie, 1982) but, in our computer modelling, we have resolution only up to a Rayleigh number of $10^{6}$. Therefore, we have held this parameter at $10^{6}$, keeping in mind that the Rayleigh number might well be higher and that a higher Rayleigh number would lead to smaller observables and shorter uplift times (if $\Delta \mathrm{T}$ is held constant). Second, the empirical relation of viscosity to temperature and pressure predicts a broad diffuse low viscosity zone underneath the plates, but the presence of melt further decreases the viscosity (Cooper and Kohlstedt, 1984). Since the presence of melt is thought to be constrained to the top $200 \mathrm{~km}$ of the mantle (McKenzie, 1984), a low viscosity zone resulting from both of these processes would have its most significant variation above $200 \mathrm{~km}$ in depth, corresponding to a low 
viscosity zone of $125 \mathrm{~km}$ in our model. Finally, we have assumed that the conducting lid is $75 \mathrm{~km}$ thick. For Hawaii, Bermuda and Cape Verde, which lie on $80 \mathrm{~m} . \mathrm{y} ., 110 \mathrm{~m} . \mathrm{y}$. and $130 \mathrm{~m} \cdot \mathrm{y} \cdot$ old plates, respectively, this value is appropriate. However, for the Marquesas swell, which lies on $45 \mathrm{~m} . \mathrm{y}$. old lithosphere (Fischer, et al,; 1986), this thickness is acceptable but large, and we have found that a conducting lid thickness of 40-50 $\mathrm{km}$ provides a better fit to its elastic plate thickness.

\subsubsection{Hawaii}

We need only a viscosity contrast of slightly more than an order of magnitude to reproduce the peak-to-peak observables at the Hawaiian swell with our model. For a viscosity contrast of $0.04-0.06$ and a Rayleigh number of 106, the anomalies obtained from the model are: geoid $=8-$ $10 \mathrm{~m}$; topography $=1 \cdot 2-1.5^{\circ} \mathrm{km} ;$ and heat flow $=9-10 \mathrm{~mW} / \mathrm{m}^{2}$. The depth of compensation is $65-75 \mathrm{~km}$, and the elastic plate thickness is $25-30 \mathrm{~km}$ with a $450^{\circ} \mathrm{C}$ isotherm as the base of the elastic plate and $29-34 \mathrm{~km}$ with a $600{ }^{\circ} \mathrm{C}$ isotherm. The values compare favorably with those observed at Hawaii. (see Table 3.3), except for perhaps the heat flow value. Since the heat flow coverage at large ages and across the swell is not complete, however, the actual value may be higher than measured.

\subsubsection{Bermuda}

The Bermuda Rise sits off the eastern coast of North America at close to 30 degrees north. Volcanism appears to 
have begun at Bermuda close to $45 \mathrm{~m} . \mathrm{y}$. ago and to have ceased $33 \mathrm{~m} \cdot \mathrm{y}$. ago. Since there appears to have been little subsidence of the swell, it is assumed that, although there is no surface volcanism at present, the hotspot is still active underneath the swell (Detrick et al., 1986).

With a viscosity contrast of 0.03 to 0.05 , our model agrees with the measured observables at the Bermuda Rise (Table 3.3). The geoid anomaly lies between 6 and $9 \mathrm{~m}$; the topography anomaly ranges between 1.0 and $1.2 \mathrm{~m}$; the heat flow anomaly lies between 8.0 and $9.5 \mathrm{mw} / \mathrm{m}^{2}$; and the depth of compensation ranges between 55 and $70 \mathrm{~km}$.

\subsubsection{Cape Verde}

Cape Verde, one of the largest swells, sits just off the west coast of North Africa on a flow line from Bermuda across the Mid-Atlantic Ridge. It rises to a height of 2 $(+/-0.2) \mathrm{km}$ on plots of residual topography with some estimates ranging up to $2.4 \mathrm{~km}$ (McNutt, 1987). The oldest volcanics date in the lower Cretaceous (Dash et al., 1976, Stillman et al., 1982), but the bulk of the volcanics appear to have been deposited in the Neogene, 12-15 Ma, with intermittent activity to the present. Interpretations differ as to when the major uplift took place creating the swell. DSDP drill cores show that the crest of the swell went above the carbonate compensation depth around $19 \mathrm{Ma}$ (Lancelot et al., 1977), but Knill (1985) has estimated, from $40 \mathrm{Ar} /{ }^{39} \mathrm{Ar}$ measurements on alkaline basaltic clastics from Maio, that the uplift occured around $40 \mathrm{Ma}$. Since the 
time of uplift, however, there has been little subsidence, so that the source of the swell is most likely active today. With a viscosity contrast of $0.1-0.2$, the least required by any of these swells, we can reproduce the observed anomalies at Cape Verde (Table 3.3). Our geoid anomaly lies between 13 and $15 \mathrm{~m}$; the topography anomaly ranges between 1.8 and $2.2 \mathrm{~km}$; and the heat flow anomaly lies between 12 and $13.5 \mathrm{~mW} / \mathrm{m}^{2}$. The depth of compensation is also 75-80 km, and the elastic plate thickness is 27-31 $\mathrm{km}$ with a $450{ }^{\circ} \mathrm{C}$ isotherm and $31-35 \mathrm{~km}$ with a $600{ }^{\circ} \mathrm{C}$.

3.6.4 The Marquesas

The Marquesas islands in the southwest Pacific lie atop a swell which rises to near a kilometer in height. This island chain is lineated, much like Hawaii, but not in the direction of plate motion. The Marquesas swell exhibits an anomalously shallow elastic plate thickness of $21(+/-2 \mathrm{~km})$ and a depth of compensation of 40-50 km (Fischer et al., 1986).

For a viscosity contrast of 0.01 to 0.03 , the lowest required by any of these swells, the surface anomalies at the Marquesas can be easily reproduced by the model, except for the elastic plate thickness (Table 3.3). The geoid anomaly lies between 3 and $6 \mathrm{~m}$; the topography anomaly, between 800 and $1000 \mathrm{~m}$; and the heat flow anomaly, between 5.5 and $8.0 \mathrm{mw} / \mathrm{m}^{2}$. The depth of compensation is between 45 and $55 \mathrm{~km}$. 
The elastic plate thickness is near $26 \mathrm{~km}$ with a 450 ${ }^{\circ} \mathrm{C}$ isotherm as the base of the elastic plate and $31 \mathrm{~km}$ with a $600{ }^{\circ} \mathrm{C}$ isotherm $-5 \mathrm{~km}$ and $9 \mathrm{~km}$ too thick for the estimate of Fischer et al. (1986) for the Marquesas swell (21 +/- 2 $\mathrm{km})$, respectively. However, the elastic plate thickness decreases as the thickness of the conducting lid decreases. Since the Marquesas islands sit on crust with an age of 45 $\mathrm{m} . \mathrm{y}$. , the conducting lid may only extend to $40-50 \mathrm{~km}$ in depth. If we reduce the conducting lid thickness to $50 \mathrm{~km}$, the apparent elastic plate thickness is $17-23 \mathrm{~km}$ for a 450 ${ }^{\circ} \mathrm{C}$ isotherm and 21-27 km with a $600{ }^{\circ} \mathrm{C}$ isotherm, both of which are in agreement with the estimates of Fischer et al. (1986) .

\subsection{Discussion}

One implication of this study is that, since we do not disturb the physical integrity of the conducting lid and since we can still reproduce the surface observables measured at a number of swells, significant lithospheric "thinning" above a depth of $75 \mathrm{~km}$ on old oceanic lithosphere (such as at Hawaii, Bermuda and Cape Verde) or above a depth of $50 \mathrm{~km}$ at the Marquesas is not required by the data. The temperature structure is elevated above this depth, but the convective stress can be applied to the plate at depths below $75 \mathrm{~km}$.

Although we have scaled the results to the upper mantle, our results can also be applied to the case of whole 
mantle convection. Since the topographic and geoid response to the convective temperature anomalies at depth is nearly zero near the base of the fluid layers (Robinson et al., 1987a; Chapter 2) and since the principal long wavelength temperature variations are confined to the boundary layers (see Figure 3.2), the topography and geoid anomalies will reflect primarily the temperature variations in the top boundary layer. In particular, because these anomalies represent integrals of the temperature variations and the appropriate response functions, they will depend on the thickness of the top boundary layer and the temperature contrast across it. A property of high Rayleigh number convection is that the physical thickness of the top boundary layer remains constant as the depth of convection increases with all of the other parameters fixed. Therefore, since the temperature drop across the top boundary layer is determined from the prescribed heat flux, the topography and geoid anomalies as well as the depth of compensation, elastic plate thickness, and uplift time should be insensitive to the depth of the convecting layer. Another implication of these results is that, if convection in the mantle does have a widespread and longwavelength effect on the topography and geoid anomalies in the ocean basins, then it is important to correct for this effect when calculating the values for the parameters of the thermal plates. Theoretically, a reference depth-age relationship could be derived for the plates by a 
complicated average over the dominant wavelengths of the convection. In practice, such averaging is impossible, since we do not know the wavelengths or amplitude of the convection or the precise viscosity structure for the upper mantle. Parsons and Sclater (1977) tried to approximate such an analysis by eliminating the largest topography associated with swells. They then averaged over the ocean basin. However, since the upwellings are pronounced whereas the downwellings are much more diffuse in a threedimensional convective flow, this depth-age relationship could be biased to deeper values.

\subsection{Conclusions}

We have investigated the effect of a low viscosity zone on the uplift produced by a convection cell, and have applied this model to the formation of mid-plate swells. We have found that, by prescribing the viscosity structure and the heat flux alone, we can produce all of the surface observables seen at four of the most well documented swells. The viscosity structures required to produce the appropriate uplift have low viscosity layer in the uppermost mantle with a viscosity which is one to two orders of magnitude less than the viscosity of the rest of the upper mantle. We used an idealized finite element model of the material in the upper mantle to explore the effect of such a low viscosity zone on the formation of mid-plate swells. The model consists of three layers: a conducting lid, which 
overlies a low viscosity zone, which in turn overlies a constant viscosity layer. We varied the viscosity contrast, the Rayleigh number, and the layer thicknesses.

Since the low viscosity zone decreases the stress transmitted to the base of the conducting lid from the fluid flow, the surface topography decreases in magnitude as the viscosity in the low viscosity zone is decreased. Furthermore, because the geoid anomaly is the sum of the positive gravitational effects from the surface topography and the negative effects from the internal density differences, it decreases in magnitude much faster than the topography anomaly for higher viscosity contrasts. In general, the topography and geoid anomalies show an approximately linear relationship over convective flows so that, when a depth of compensation is calculated from Pratt and Airy models, the depth of compensation decreases very quickly as the viscosity contrast increases. In fact, the depth of compensation can appear to be well inside the conducting lid for sufficiently large viscosity contrasts. Because the temperature structure is elevated underneath the swell the elastic plate thickness decreases in the presence of convection. Since this model reflects a convective time scale, it results in very short uplift times. In particular, the low viscosity zone increases the rate that the material can flow underneath the conducting lid, so that it decreases the uplift rate. 
We find that we can produce the topography, geoid and heat flow anomalies, as well as the depths of compensation and elastic plate thicknesses observed at the swells associated with the Hawaiian islands, Bermuda, the Marquesas islands and Cape Verde. Our preferred model has a Rayleigh number of $10^{6}$ and a low viscosity layer thickness of $125 \mathrm{~km}$. This Rayleigh number is consistent with estimates of the Rayleigh number for the upper mantle (McKenzie et al., 1974; Houseman and McKenzie, 1984), and the low viscosity layer thickness is consistent with estimates from studies of small scale convective anomalies in the oceanic geoid (Haxby and Weissel, 1986; Robinson and Parsons, 1987; Chapter 5), seismic tomography (Forsyth, 1977; Weilandt and Knopoff, 1982) and melt production (e.g. McKenzie, 1984). The differences between the swells can then be accommodated by changing the viscosity in the low viscosity zone. At Cape Verde a viscosity contrast of one order of magnitude or slightly less is required. At Hawaii, Bermuda and the Marquesas, low viscosity zones representing a one to two order of magnitude change in viscosity are required. The viscosity contrast underneath the swells decrease as their lithospheric age increases. This trend is consistent with theoretical predictions of the magnitude of the low viscosity zone as it ages from the temperature and pressure dependence of viscosity and the thermal evolution of the oceanic plates. 
Although the above model is preferred because it is consistent with the results of other studies (Forsyth, 1977; Weilandt and Knopoff, 1982; McKenzie, 1984; Craig and McKenzie, 1986; Robinson et al., 1987b; Robinson and Parsons, 1987), this study cannot uniquely constrain the Rayleigh number, low viscosity layer thickness or viscosity contrast, because these parameters often have similar effects on the observables. For example, the Rayleigh number could be greater than $10^{6}$. The uplift time would then be shorter and the observables smaller, so that smaller viscosity contrasts would be required at depth. Also, since there exist no shorter wavelength features at swells that can be ascribed to convection in a low viscosity zone, it is very difficult to directly constrain the thickness of the low viscosity zone beneath them. However, the depth of compensation is sensitive in particular to the viscosity contrast and, from the depths of compensation, we can estimate within reasonable errors the viscosity contrast at depth. Given these viscosity contrasts, the Rayleigh numbers and low viscosity layer thicknesses can be further constrained and are found to be consistent with estimates from the literature.

In conclusion, the uplift seen at swells can be explained by a convection model, where we prescribe only the viscosity structure and the heat flux at the base of the fluid. The presence of the low viscosity zone immediately beneath the mechanical portion of the plate is critical in 
the application of this model to mid-plate swells. The viscosities that are required range from 0.2 to 0.01 of the viscosity in the lower portions of the upper mantle, and are very easily produced by the temperature and pressure variations that thought to be present in the upper mantle.

\section{Acknowledgments}

We would like to thank Karen Fischer for helpful discussions. This work has been supported by NASA Geodynamics program grant NAG5-415, and National science Foundation grant OCE-8516298. We would also like to thank Tom Jordan for making computing time on the Alliant FX/1 available to us. 


\section{Table 3.1}

Variable Description

d

F

$\rho_{\circ}$

$\rho_{\mathrm{W}}$

go

$\kappa$

$\alpha$

$\mathrm{C}_{\mathrm{p}}$

$\Delta \mathrm{T}$

E

v

$\mathrm{k}_{\mathrm{C}}$

$\mathrm{N}_{\mathrm{O}}$

$G_{0}$

$\mathrm{H}_{\mathrm{O}}$

$q_{0}$

$$
\text { depth scale }
$$

flux on the bottom boundary

average mantle density

density of water

surface gravitational acceleration $10 \mathrm{~m} / \mathrm{s}^{2}$

average mantle thermal diffusivity $10^{-6} \mathrm{~m}^{2} / \mathrm{s}$

average mantle thermal

expansion coefficient

specific heat

conductive temperature across

the depth of the box

young's modulus

Poisson's ratio

thermal conductivity

scaling value for geoid

scaling value for gravity

scaling value for topography

scaling value for heat flow
Value

$600 \mathrm{~km}$

1 H. F. U.

$3300 \mathrm{~kg} / \mathrm{m}^{3}$

$1000 \mathrm{~kg} / \mathrm{m}^{3}$

$2 \times 10^{-5}{ }^{\circ} \mathrm{C}^{-1}$

$900 \mathrm{~K} / \mathrm{kg} \cdot{ }^{\circ} \mathrm{C}$

$8656{ }^{\circ} \mathrm{C}$

$8 \times 10^{10} \mathrm{~N} / \mathrm{m}^{2}$

0.25

$3.0 \mathrm{~W} / \mathrm{m} \cdot{ }^{\circ} \mathrm{C}$

$8414 \mathrm{~m}$

$0.1402 \mathrm{~m} / \mathrm{s}$

$103,872 \mathrm{~m}$

$42.8 \mathrm{~mW} / \mathrm{m}^{2}$ 
Table 3.2

\begin{tabular}{|c|c|c|c|c|c|c|}
\hline RUN & $\begin{array}{l}\text { LID } \\
\text { THICK. }\end{array}$ & $\begin{array}{l}\text { LVI } \\
\text { THICK. }\end{array}$ & $\begin{array}{l}\text { RAYLEIGH } \\
\text { NUMBER }\end{array}$ & $\mu_{t} / \mu_{b}$ & $\begin{array}{l}\text { GRID } \\
\text { SIZE }\end{array}$ & $\begin{array}{l}\text { FIGURE } \\
\text { NUMBER }\end{array}$ \\
\hline $\begin{array}{l}1 . \\
2 . \\
3 . \\
4 .\end{array}$ & $\begin{array}{l}0.125 \\
0.125 \\
0.125 \\
0.125\end{array}$ & $\begin{array}{l}0.0 \\
0.21 \\
0.21 \\
0.21\end{array}$ & $\begin{array}{l}1.0 \times 10^{4} \\
1.0 \times 10^{4} \\
1.0 \times 10^{4} \\
1.0 \times 10^{4}\end{array}$ & $\begin{array}{l}1.0 \\
0.1 \\
0.01 \\
0.001\end{array}$ & $\begin{array}{l}24 \times 27 \\
24 \times 32 \\
24 \times 42 \\
24 \times 42\end{array}$ & $\begin{array}{l}3.5,6,13,14 \\
3.5,6,13,14 \\
3.3,4-6,14 \\
3.5,6\end{array}$ \\
\hline $\begin{array}{l}5 . \\
6 . \\
7 .\end{array}$ & $\begin{array}{l}0.125 \\
0.125 \\
0.125\end{array}$ & $\begin{array}{l}0.0 \\
0.21 \\
0.21\end{array}$ & $\begin{array}{l}3.2 \times 10^{4} \\
3.2 \times 10^{4} \\
3.2 \times 10^{4}\end{array}$ & $\begin{array}{l}1.0 \\
0.1 \\
0.01\end{array}$ & $\begin{array}{l}24 \times 27 \\
24 \times 32 \\
24 \times 42\end{array}$ & $\begin{array}{l}3.5,6 \\
3.5,6 \\
3.5,6\end{array}$ \\
\hline $\begin{array}{l}8 . \\
9 . \\
10 . \\
11 . \\
12 . \\
13 . \\
14 .\end{array}$ & $\begin{array}{l}0.125 \\
0.125 \\
0.125 \\
0.125 \\
0.125 \\
0.125 \\
0.125\end{array}$ & $\begin{array}{l}0.0 \\
0.21 \\
0.21 \\
0.21 \\
0.21 \\
0.21 \\
0.21\end{array}$ & $\begin{array}{l}1.0 \times 10^{5} \\
1.0 \times 10^{5} \\
1.0 \times 10^{5} \\
1.0 \times 10^{5} \\
1.0 \times 10^{5} \\
1.0 \times 10^{5} \\
1.0 \times 10^{5}\end{array}$ & $\begin{array}{l}1.0 \\
0.1 \\
0.075 \\
0.050 \\
0.025 \\
0.01 \\
0.001\end{array}$ & $\begin{array}{l}24 \times 27 \\
24 \times 42 \# \\
24 \times 42 \\
24 \times 42 \\
24 \times 42 \\
24 \times 42 \# \\
24 \times 42 \#\end{array}$ & $\begin{array}{l}3.2,4-6,10-14 \\
3.2-14 \\
3.5,6,10-14 \\
3.5,6,10-14 \\
3.5,6,10-14 \\
3.2,4-6,10-14 \\
3.5,6,10-14\end{array}$ \\
\hline $\begin{array}{l}15 . \\
16 . \\
17 .\end{array}$ & $\begin{array}{l}0.125 \\
0.125 \\
0.125\end{array}$ & $\begin{array}{l}0.0 \\
0.21 \\
0.21\end{array}$ & $\begin{array}{l}3.2 \times 10^{5} \\
3.2 \times 10^{5} \\
3.2 \times 10^{5}\end{array}$ & $\begin{array}{l}1.0 \\
0.1 \\
0.01\end{array}$ & $\begin{array}{l}24 \times 27 \\
24 \times 42 \\
24 \times 42\end{array}$ & $\begin{array}{l}3.5,6,10,11 \\
3.5,6,10,11 \\
3.5,6,10,11\end{array}$ \\
\hline $\begin{array}{l}18 . \\
19 . \\
20 .\end{array}$ & $\begin{array}{l}0.125 \\
0.125 \\
0.125\end{array}$ & $\begin{array}{l}0.0 \\
0.21 \\
0.21\end{array}$ & $\begin{array}{l}1.0 \times 10^{6} \\
1.0 \times 10^{6} \\
1.0 \times 10^{6}\end{array}$ & $\begin{array}{l}1.0 \\
0.1 \\
0.01\end{array}$ & $\begin{array}{l}24 \times 27 \\
24 \times 42 \\
24 \times 42\end{array}$ & $\begin{array}{l}3.5,6,10-14 \\
3.3,4,5,6,10-14 \\
3.5,6,10-12,14\end{array}$ \\
\hline $\begin{array}{l}21 . \\
22 . \\
23 \\
24 .\end{array}$ & $\begin{array}{l}0.125 \\
0.125 \\
0.125 \\
0.125\end{array}$ & $\begin{array}{l}0.083 \\
0.083 \\
0.083 \\
0.083\end{array}$ & $\begin{array}{l}1.0 \times 10^{4} \\
1.0 \times 10^{5} \\
1.0 \times 10^{5} \\
1.0 \times 10^{5}\end{array}$ & $\begin{array}{l}0.1 \\
0.1 \\
0.01 \\
0.001\end{array}$ & $\begin{array}{l}24 \times 29 \\
24 \times 29 \\
24 \times 29 \\
24 \times 29\end{array}$ & $3.4,3.7$ \\
\hline $\begin{array}{l}25 . \\
26 . \\
27 .\end{array}$ & $\begin{array}{l}0.125 \\
0.125 \\
0.125\end{array}$ & $\begin{array}{l}0.5 \\
0.5 \\
0.5\end{array}$ & $\begin{array}{l}1.0 \times 10^{4} \\
1.0 \times 10^{5} \\
1.0 \times 10^{5}\end{array}$ & $\begin{array}{l}0.1 \\
0.1 \\
0.01\end{array}$ & $\begin{array}{l}24 \times 40 \\
24 \times 40 \# \\
24 \times 40 \#\end{array}$ & 3.4 .3 .7 \\
\hline $\begin{array}{l}28 . \\
29 .\end{array}$ & $\begin{array}{l}0.125 \\
0.125\end{array}$ & $\begin{array}{l}0.75 \\
0.75\end{array}$ & $\begin{array}{l}1.0 \times 10^{4} \\
1.0 \times 10^{5}\end{array}$ & $\begin{array}{l}0.1 \\
0.1\end{array}$ & $\begin{array}{l}24 \times 46 \\
24 \times 46\end{array}$ & 3.4 \\
\hline $\begin{array}{l}30 . \\
31 . \\
32 .\end{array}$ & $\begin{array}{l}0.0 \\
0.042 \\
0.083\end{array}$ & $\begin{array}{l}0.0 \\
0.0 \\
0.0\end{array}$ & $\begin{array}{l}1.0 \times 10^{5} \\
1.0 \times 10^{5} \\
1.0 \times 10^{5}\end{array}$ & $\begin{array}{l}1.0 \\
1.0 \\
1.0\end{array}$ & $\begin{array}{l}24 \times 24 \\
24 \times 25 \\
24 \times 26\end{array}$ & \\
\hline
\end{tabular}


Table 3.3

\begin{tabular}{|c|c|c|c|c|}
\hline $\begin{array}{l}\text { GEOID } \\
\text { ANOMALY } \\
\text { (in m) }\end{array}$ & $\frac{\text { HAWAII }}{6-10^{1}}$ & $\frac{\text { BERMUDA }}{6-10^{7}}$ & $\frac{\text { CAPE VERDE }}{11-15^{9}, 10}$ & $\frac{\text { MARQUESAS }}{4-811,12}$ \\
\hline $\begin{array}{l}\text { TOPOGRAPHY } \\
\text { ANOMALY\# : } \\
\text { (in } \mathrm{km} \text { ) }\end{array}$ & $1.0-1.4^{2}$ & $0.8-1.2^{7}$ & $1.8-2 \cdot 2^{9,10}$ & $0.8-1.2^{11,12}$ \\
\hline $\begin{array}{l}\text { HEAT FLOW } \\
\text { ANOMALY } \\
\left(\text { in } \mathrm{mW} / \mathrm{m}^{2}\right)\end{array}$ & $5-9^{3}$ & $8-10^{7}$ & $12-20^{9}$ & ----- \\
\hline $\begin{array}{l}\text { DEPTH OF } \\
\text { COMPENSATION: } \\
\text { (in } \mathrm{km} \text { ) }\end{array}$ & $60-80^{4,5}$ & $40-70^{4,8}$ & $59-79^{10}$ & $35-55^{12}$ \\
\hline $\begin{array}{l}\text { ELASTIC PLATE } \\
\text { THICKNESS : } \\
\text { (in km) }\end{array}$ & $25-35^{1,5,6}$ & $-\cdots--$ & $24-32^{10}$ & $19-23^{12}$ \\
\hline $\begin{array}{l}\text { UPLIFT TIME : } \\
(\text { in } m \cdot y \cdot)\end{array}$ & $<10^{4,5}$ & $-\cdots-$ & $\ldots$ & $\ldots$ \\
\hline \multicolumn{5}{|c|}{ \# - peak-to-peak anomalies } \\
\hline \multicolumn{5}{|c|}{$\begin{array}{l}\text { References: 1-estimated from watts (1976); 2-estimated from } \\
\text { Haxby and Weissel (1986); 3-Von Herzen et al. (1982); 4- } \\
\text { Crough (1978); 5-McNutt and Shure (1986); 6-Haxby and } \\
\text { Turcotte (1978); 7-estimated from Detrick et al. (1986); 8- } \\
\text { Watts (1978); 9-estimated from Courtney and white (1986); } \\
\text { 10-estimated from McNutt (1987); 11-Crough and Jarrard } \\
\text { (1981); 12-Fischer et al. (1986). }\end{array}$} \\
\hline
\end{tabular}




\section{Eigure Captions}

Figure 3.1: Geometry of the model, u is the horizontal velocity and $w$ is the vertical velocity. $T$ is the temperature and $\sigma$ is the shear stress." "C" is a constant; "b" is the depth of the entire model; and " $a$ " is the depth of the constant viscosity layer.

Figure 3.2: Steady state temperature plots for runs where the Rayleigh number is $10^{5}$, the low viscosity layer thickness is 0.21 and the conducting lid thickness is 0.125 . All of the temperature structures were interpolated onto a $49 \times 55$ grid before contouring. The viscosity contrast, $\mu_{C}$, varies: (a) no viscosity contrast (run 8 ); (b) $\mu_{\mathrm{t}} / \mu_{\mathrm{b}}=0.1$ $(\operatorname{run} 9) ;(c) \mu_{t} / \mu_{b}=0.01(\operatorname{run} 13) ;(d) \mu_{t} / \mu_{b}=0.001 \quad(\operatorname{run} 14)$ (where the numbers refer to Table 3.2). The temperature contour intervals are, to the nearest $5{ }^{\circ} \mathrm{C}$ : (a) $150{ }^{\circ} \mathrm{C}$; (b) $135^{\circ} \mathrm{C} ;$ (c) $120^{\circ} \mathrm{C} ;$ (d) $115^{\circ} \mathrm{C}$. The tick marks on the sidewalls mark the boundaries of the low viscosity zone.

Figure 3.3: Steady state temperature plots for runs where the viscosity contrast is 0.1 , the low viscosity layer thickness is 0.21 and the conducting lid thickness is 0.125 . All of the temperature structures were interpolated onto a $49 \times 55$ grid before contouring. The Rayleigh number varies: (a) $R a=10^{4}$ (run 2); (b) $R a=10^{5}$ (run 9); (c) $R a=10^{6}$ (run 19) (where the numbers refer to Table 3.2 ). The temperature 
contour intervals are: (a) $160{ }^{\circ} \mathrm{C} ;$ (b) $120^{\circ} \mathrm{C} ;$ (c) $100{ }^{\circ} \mathrm{C}$; and the tick marks mark the boundaries of the low viscosity zone.

Eigure 3.4: Mean temperature (nondimensional) plotted versus $z^{\prime}$ for runs (a) where we have varied the viscosity contrast: 8 (line), 9 (long dash), 13 (medium dash) and 14 (short dash); (b) where we have varied the Rayleigh number: 2 (line), 9 (long dash) and 19 (medium dash); (c) where we have varied the low viscosity layer thickness: 22 (Iine), 8 (Iong dash), 26 (medium dash), and 29 (short dash). (The numbers refer to Table 3.2 .)

Figure 3.5: The $\log$ of the top boundary layer thickness plotted versus the log of the viscosity contrast for the runs with a layer thickness of 0.21 . The runs with the same Rayleigh number and a top layer thickness of 0.21 (125 km) are all joined. The circles are for a Rayleigh number of $10^{4}$; the triangles are for $\mathrm{Ra}=3.2 \times 10^{4}$; the plus signs are for $R a=10^{5}$; the crosses are for $R a=3.2 \times 10^{5}$; and the diamonds are for $\mathrm{R} a=10^{6}$.

Eigure 3.6: The log of the average temperature of the model plotted versus the $\log$ of the viscosity contrast for the runs with a layer thickness of 0.21 . The runs with the same Rayleigh number and a top layer thickness of 0.21 (125 km) are all joined. The circles are for a Rayleigh number of 
$10^{4}$; the triangles are for $\mathrm{Ra}=3.2 \times 10^{4}$; the plus signs are for $\mathrm{Ra}=10^{5}$; the crosses are for $\mathrm{Ra}=3.2 \times 10^{5}$; and the diamonds are for $\mathrm{Ra}=10^{6}$.

Figure 3.7: Steady state temperature plots for runs in which the viscosity contrast equals 0.1 and the Rayleigh number equals $10^{5}$ and where the low viscosity layer thickness varies: (a) the thickness equals 0.083 (run 22); (b) the thickness equals 0.21 (run 9); (c) the thickness equals 0.5 (run 26) (where the numbers refer to Table 3.2). The temperature contour intervals are at every $135{ }^{\circ} \mathrm{C}$, and the tick marks mark the boundaries of the low viscosity zone. All of the temperature structures were interpolated onto a $49 \times 55$ grid before contouring.

Figure 3.8: An example of the variation of the peak-to-peak topography anomaly with time during a replenishment calculation with the Rayleigh number at $10^{5}$, the viscosity contrast at 0.1 and the top layer thickness at 0.21 (125 km) (run 9).

Figure 3.9: Examples of the steady state nondimensional (a) topography; (b) geoid; and (c) heat flow anomalies for a calculation with the Rayleigh number at $10^{5}$, the viscosity contrast at 0.1 and the top layer thickness at 0.21 (125 km) (run 9, as labeled in Table 3.2). The appropriate scaling 
factors for these anomalies are: geoid, $\mathrm{N}_{\mathrm{O}}=8414 \mathrm{~m}$; topography, $h_{0}=103,872 \mathrm{~m}$; heat flow, $q_{0}=42.8 \mathrm{~mW} / \mathrm{m}^{2}$.

Eigure 3.10: The peak-to-peak nondimensional values of the geoid anomalies plotted versus the viscosity contrast for runs where the layer thickness equals 0.21 . Runs with a Rayleigh number of $10^{5}$ are denoted with circles, $3.2 \times 10^{5}$ with triangles and $10^{6}$ with plus signs.

Figure 3.11: The peak-to-peak nondimensional values of the topography anomalies plotted versus the viscosity contrast for runs where the layer thickness equals 0.21 . Runs with a Rayleigh number of $10^{5}$ are denoted with circles, $3.2 \times 10^{5}$ with triangles and $10^{6}$ with plus signs.

Eigure 3.12: The peak-to-peak nondimensional values of the heat flow anomalies plotted versus the viscosity contrast for runs where the layer thickness equals 0.21 . Runs with a Rayleigh number of $10^{4}$ are denoted with circles, $10^{5}$ with triangles and $10^{6}$ with plus signs.

Figure 3.13: The depth of compensation plotted versus the viscosity contrast for runs where the layer thickness equals 0.21 . 
Figure 3.14: The replenishment time, or "rise time", plotted versus the viscosity contrast for runs where the layer thickness equals 0.21 . 


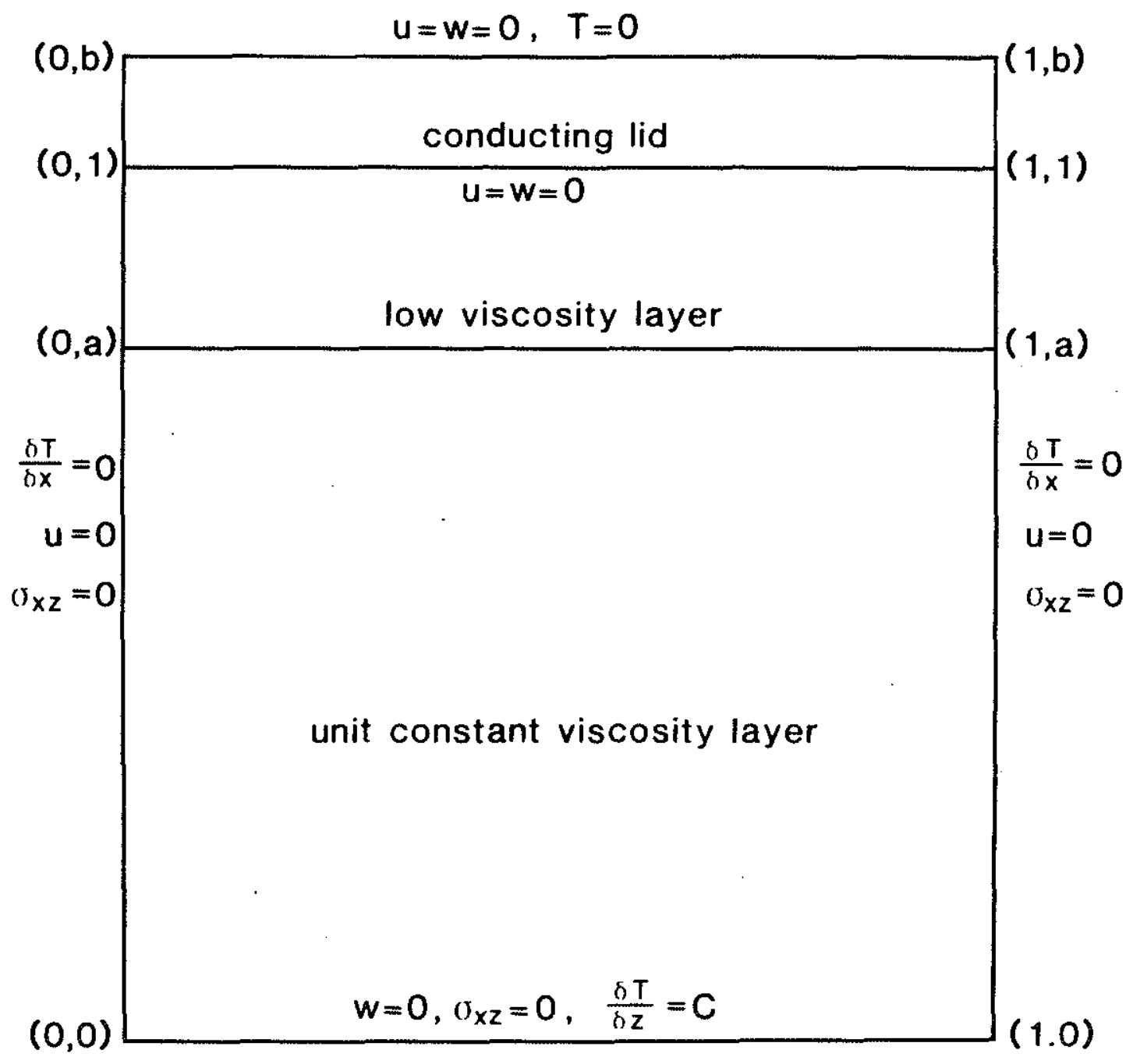

Figure 3.1 


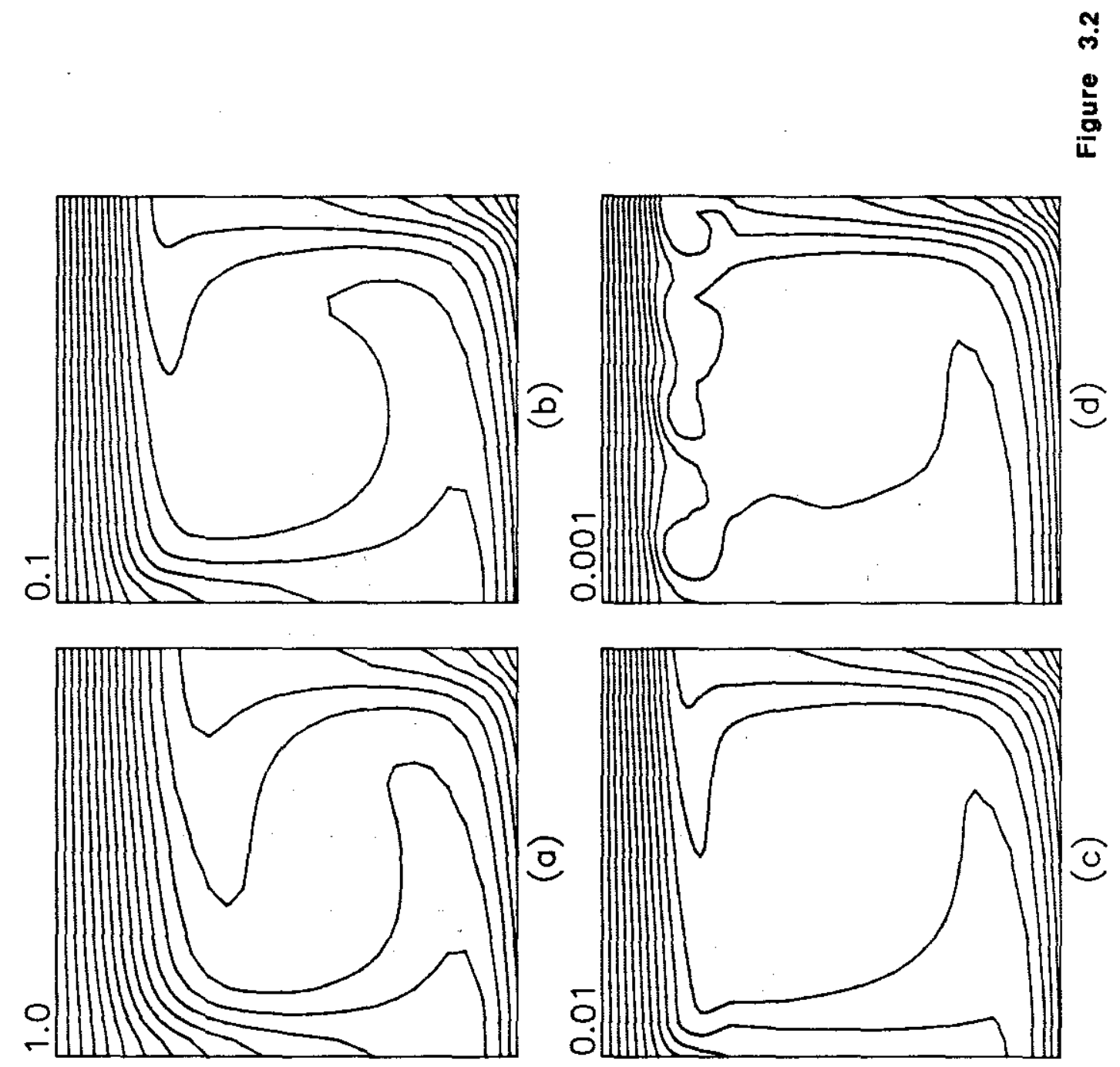




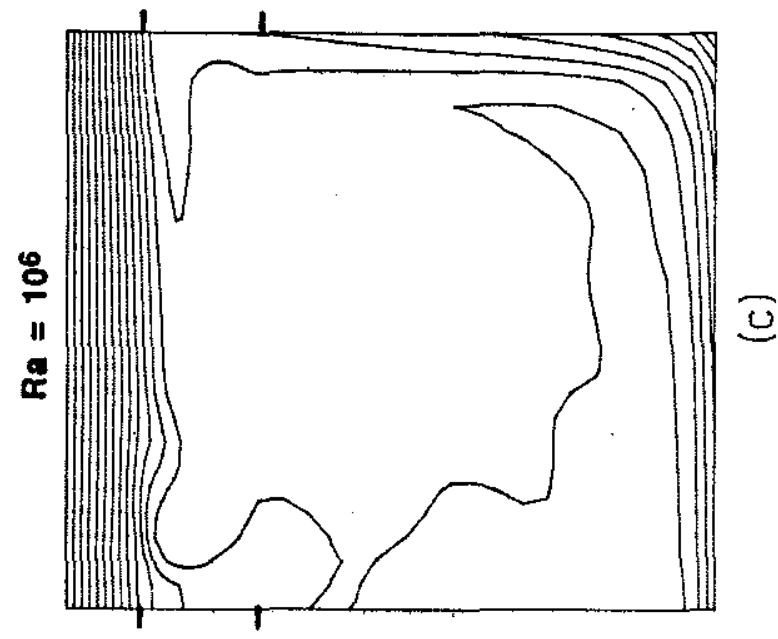

年
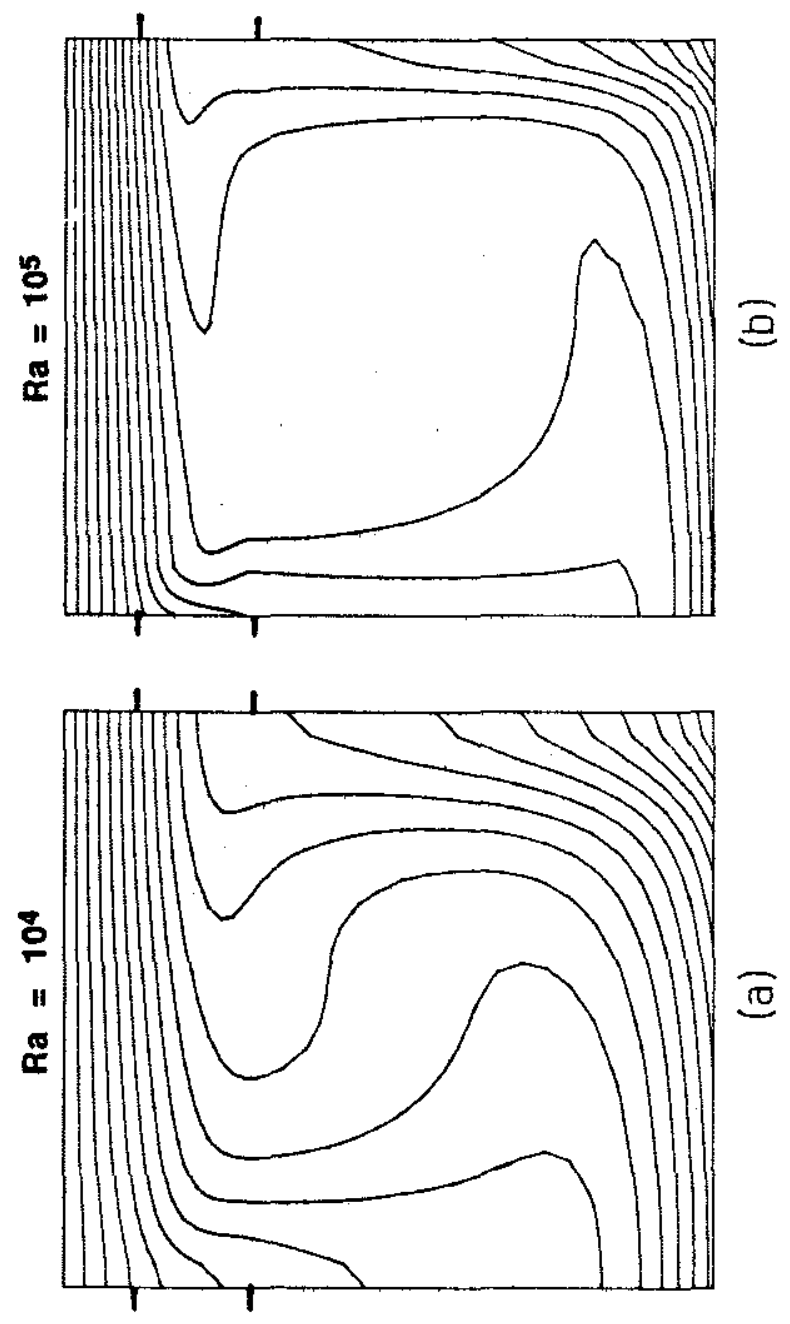
Mean $T$.

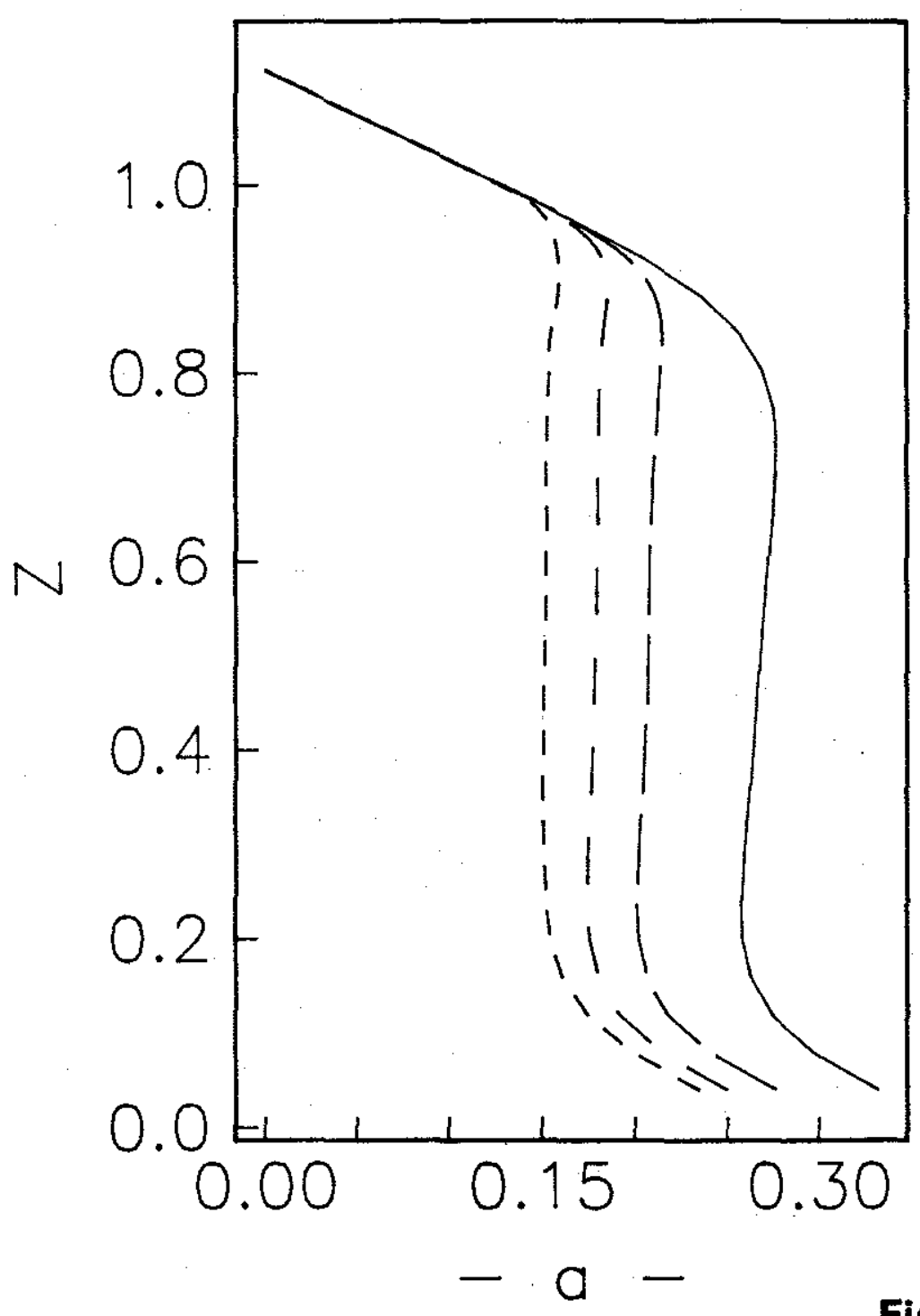

Figure $3.4 a$ 


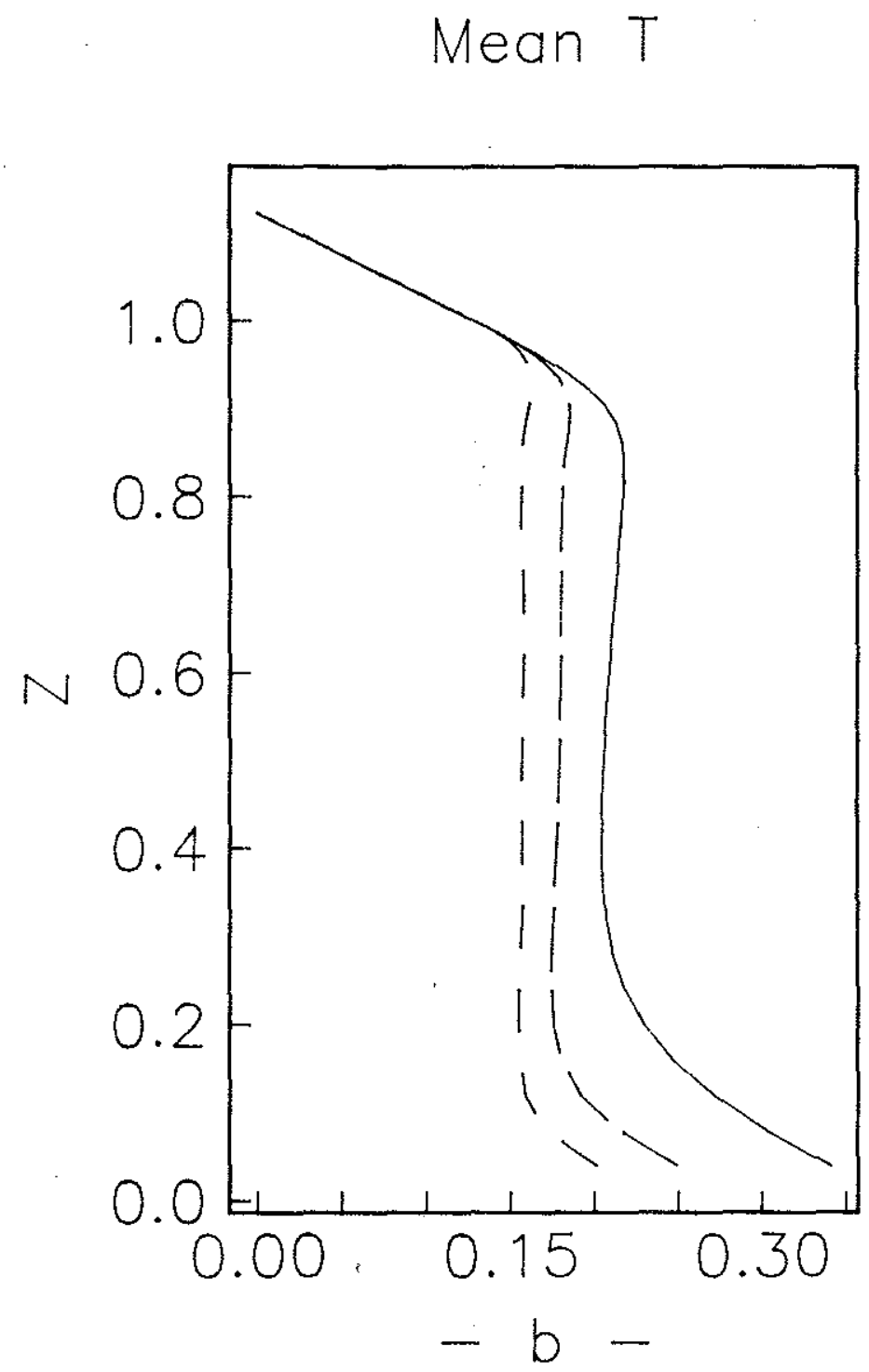

Figure $3.4 b$ 


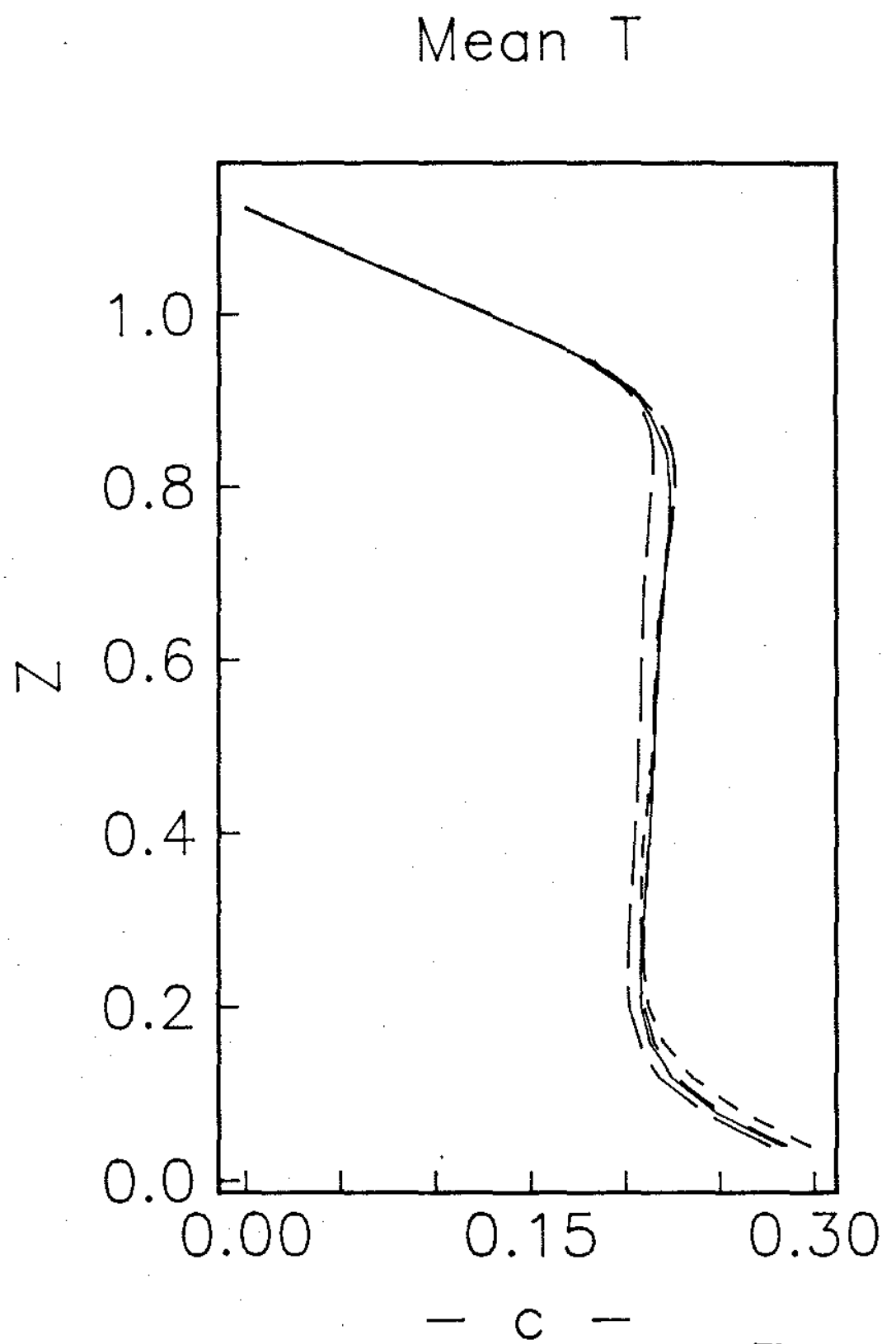

Figure $3.4 \mathrm{c}$ 


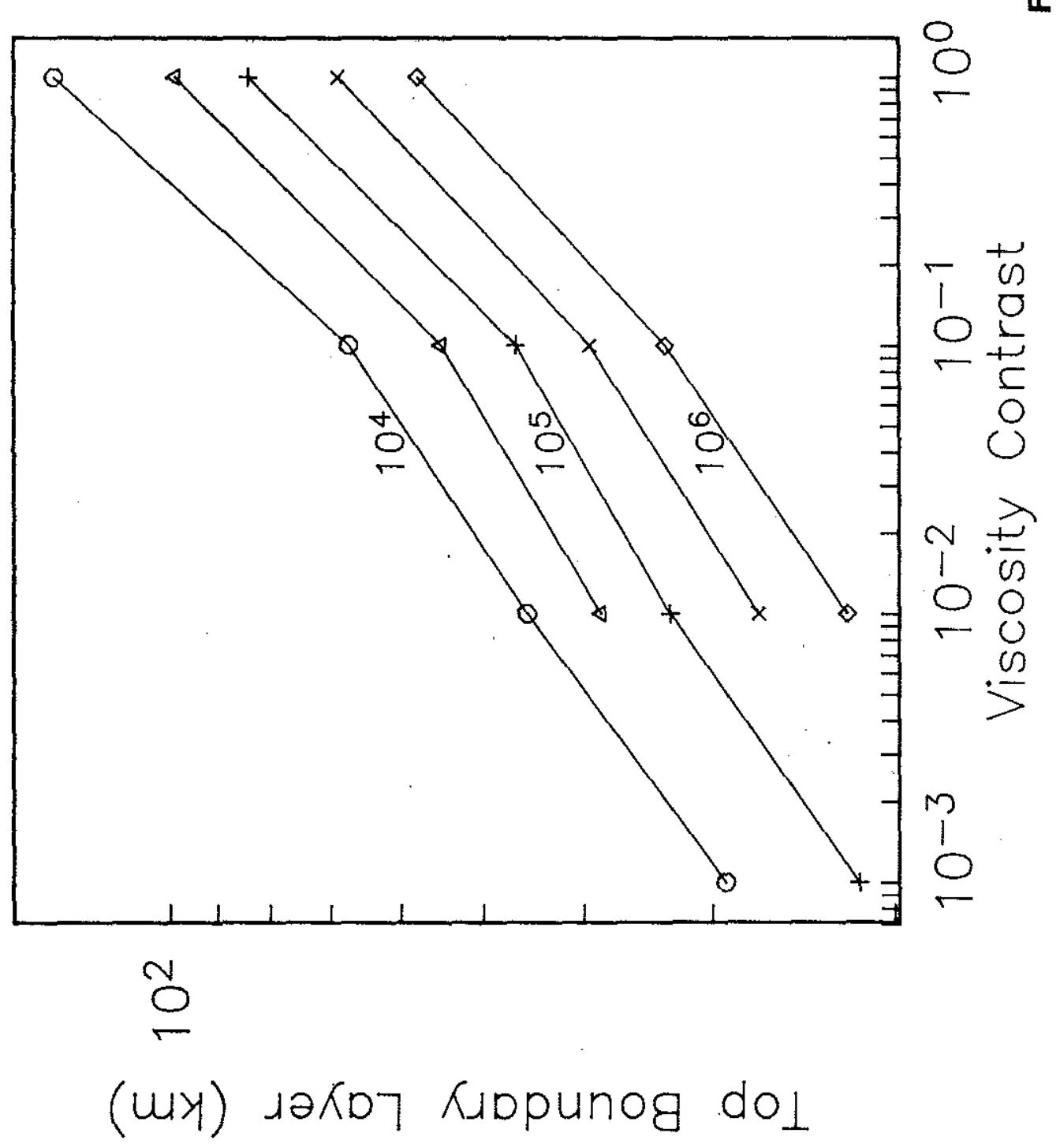




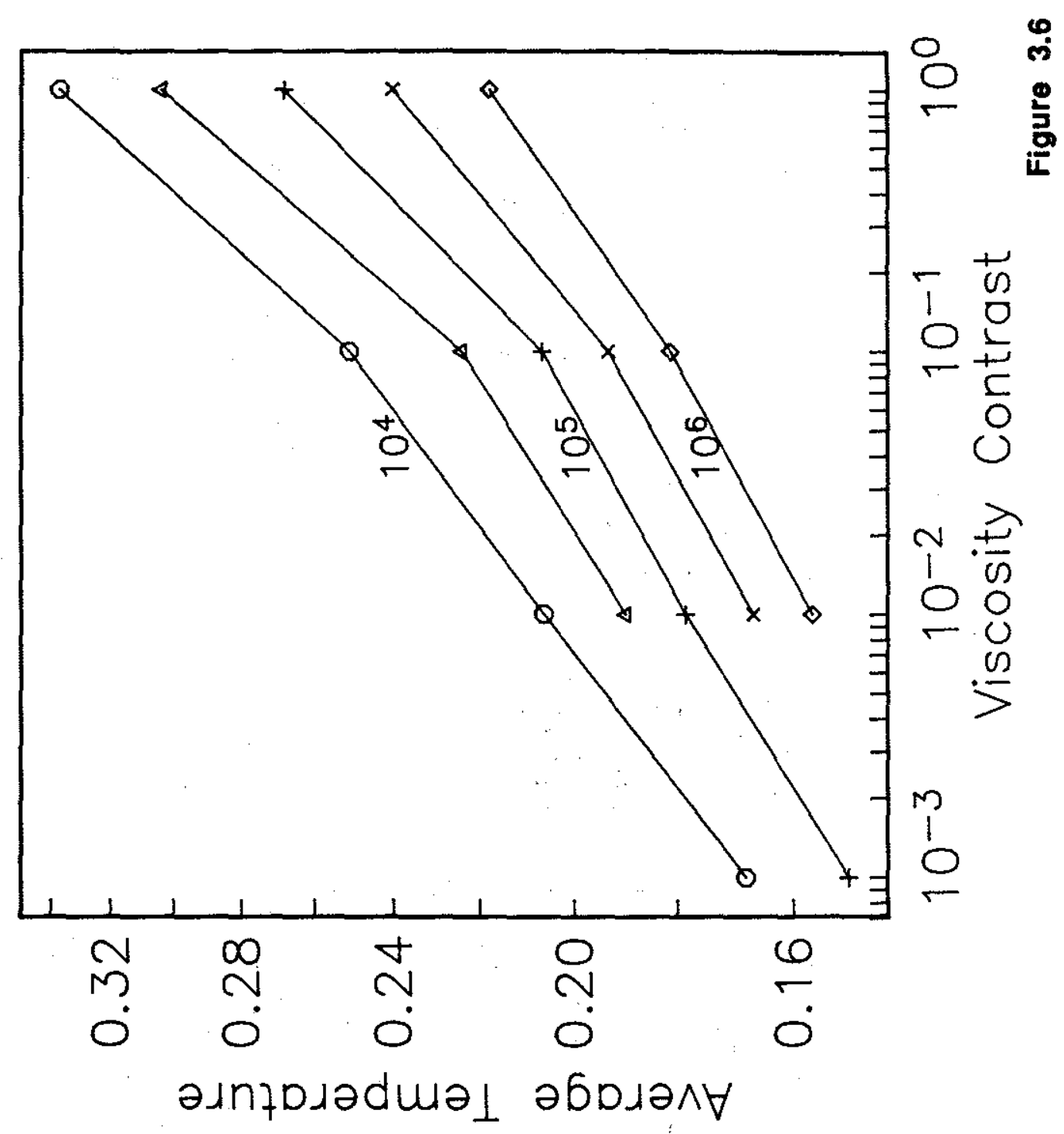



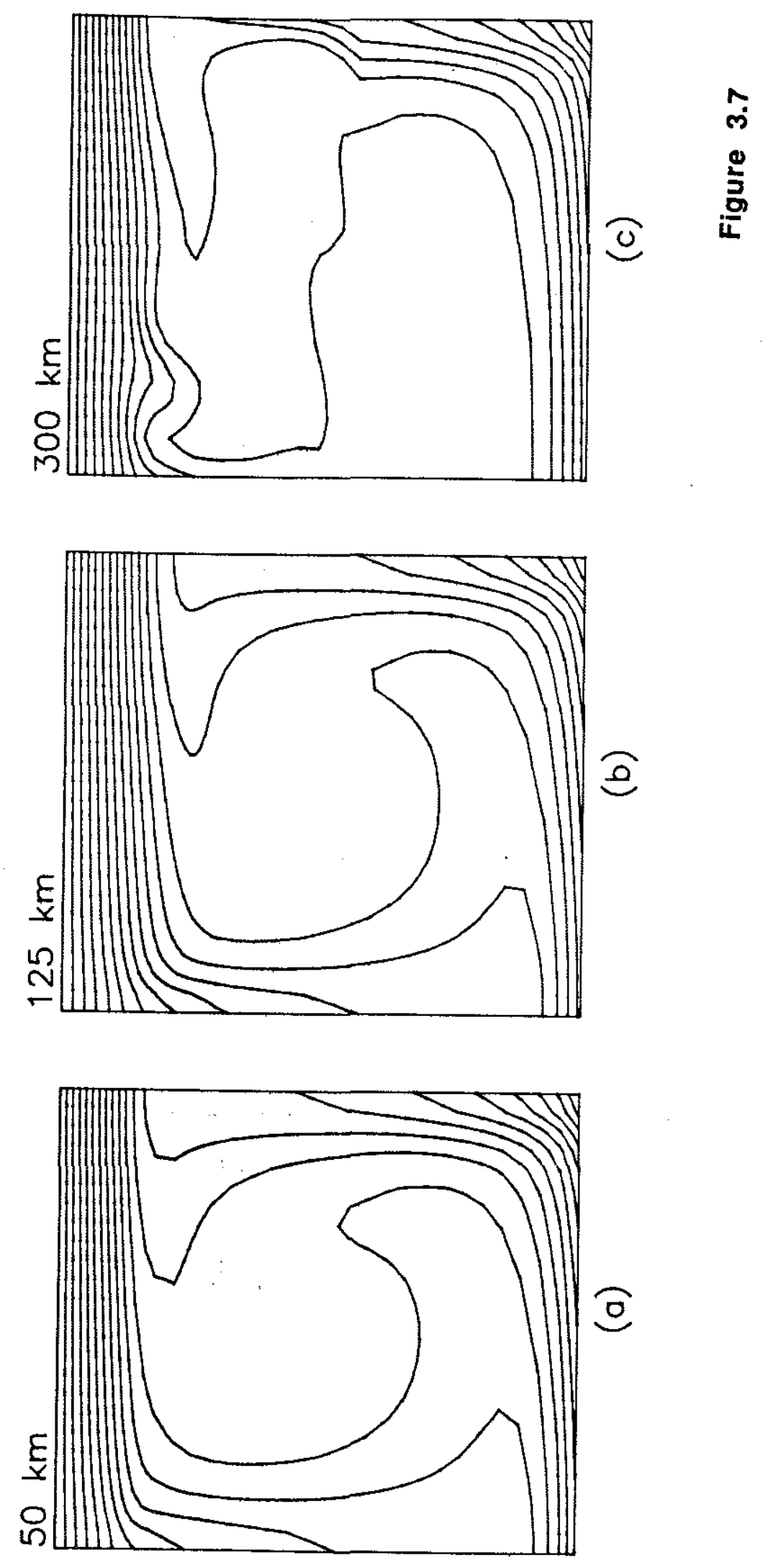


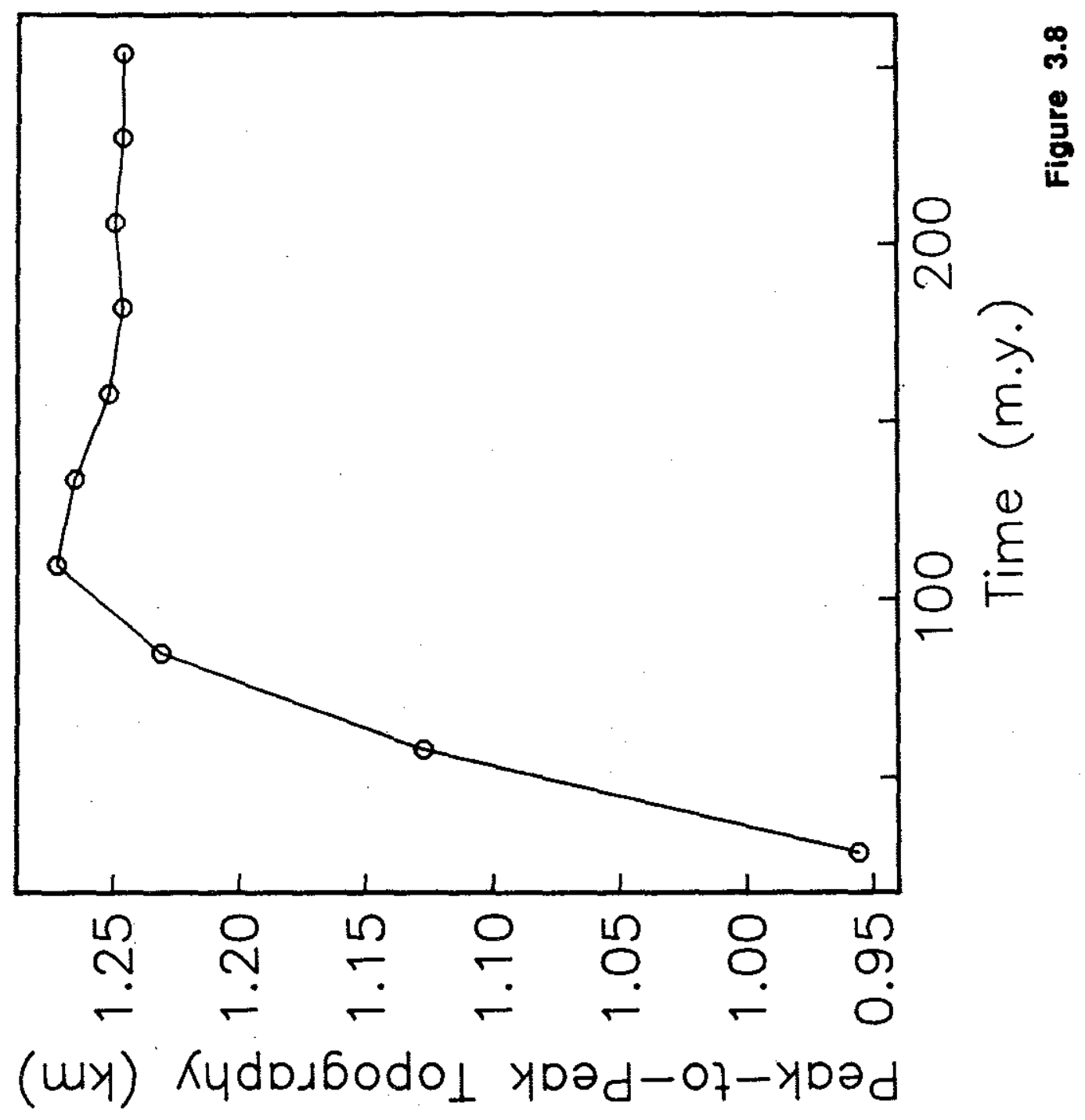


DISTANCE
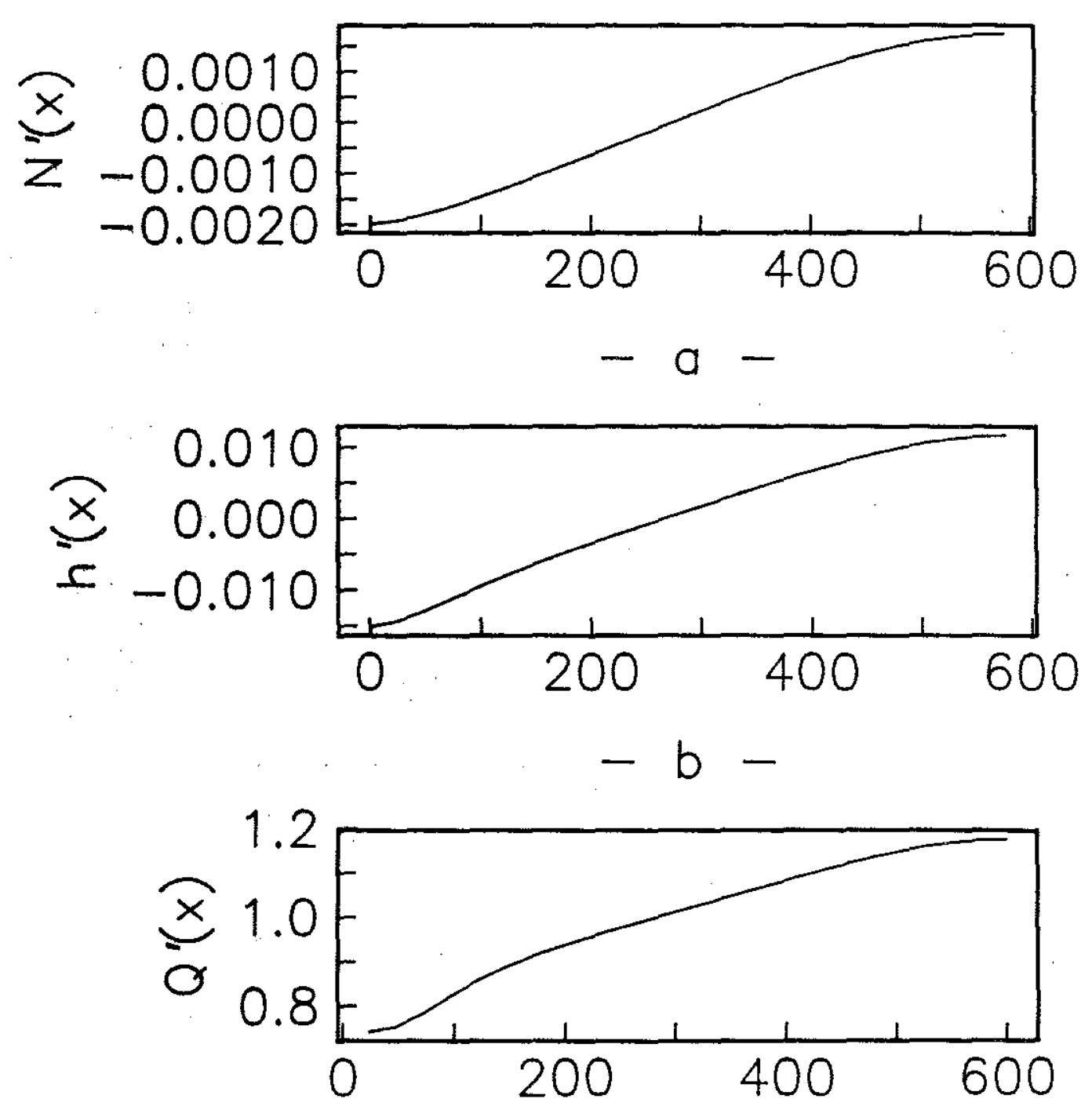

- C -

Figure 3.9 


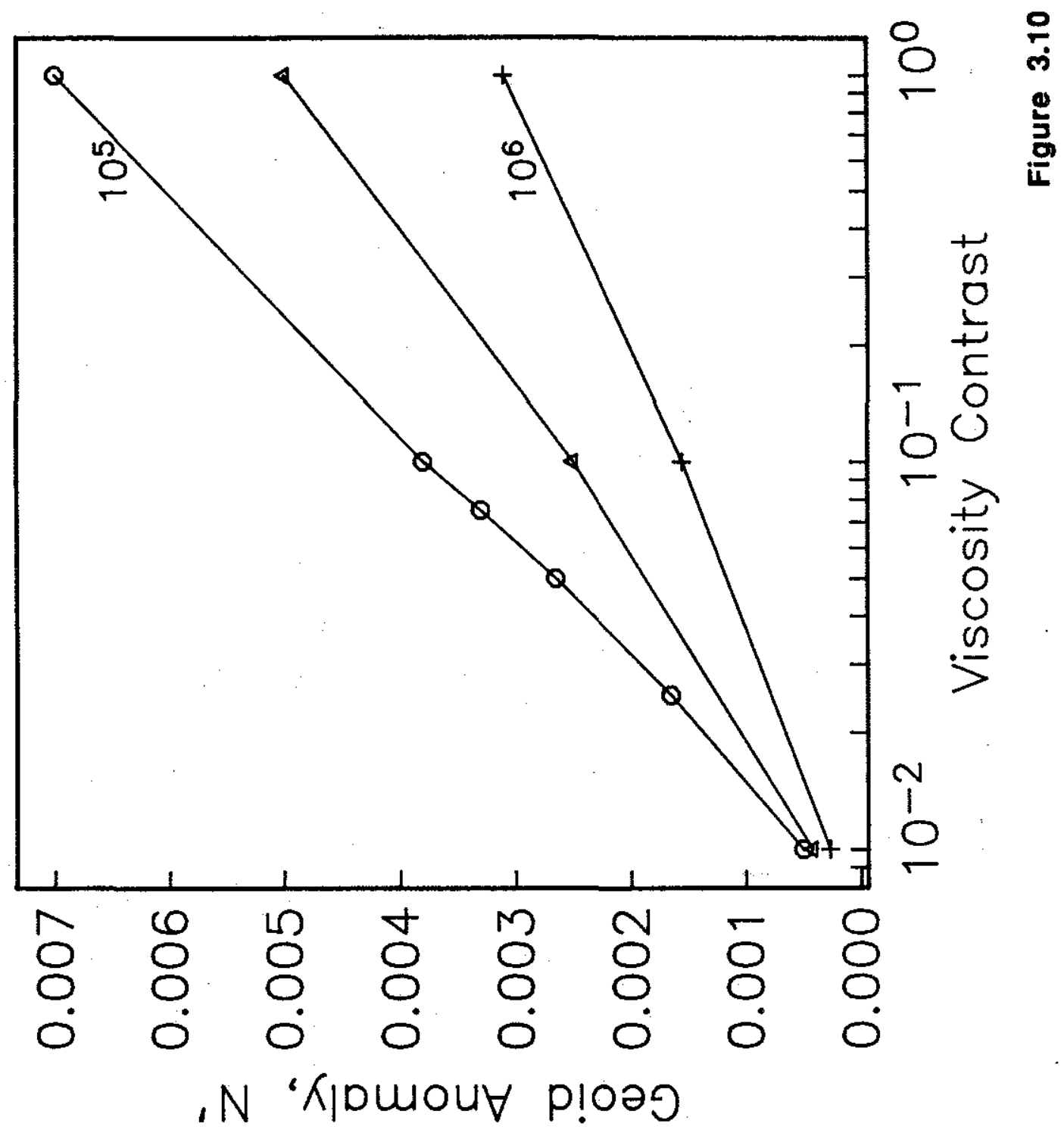




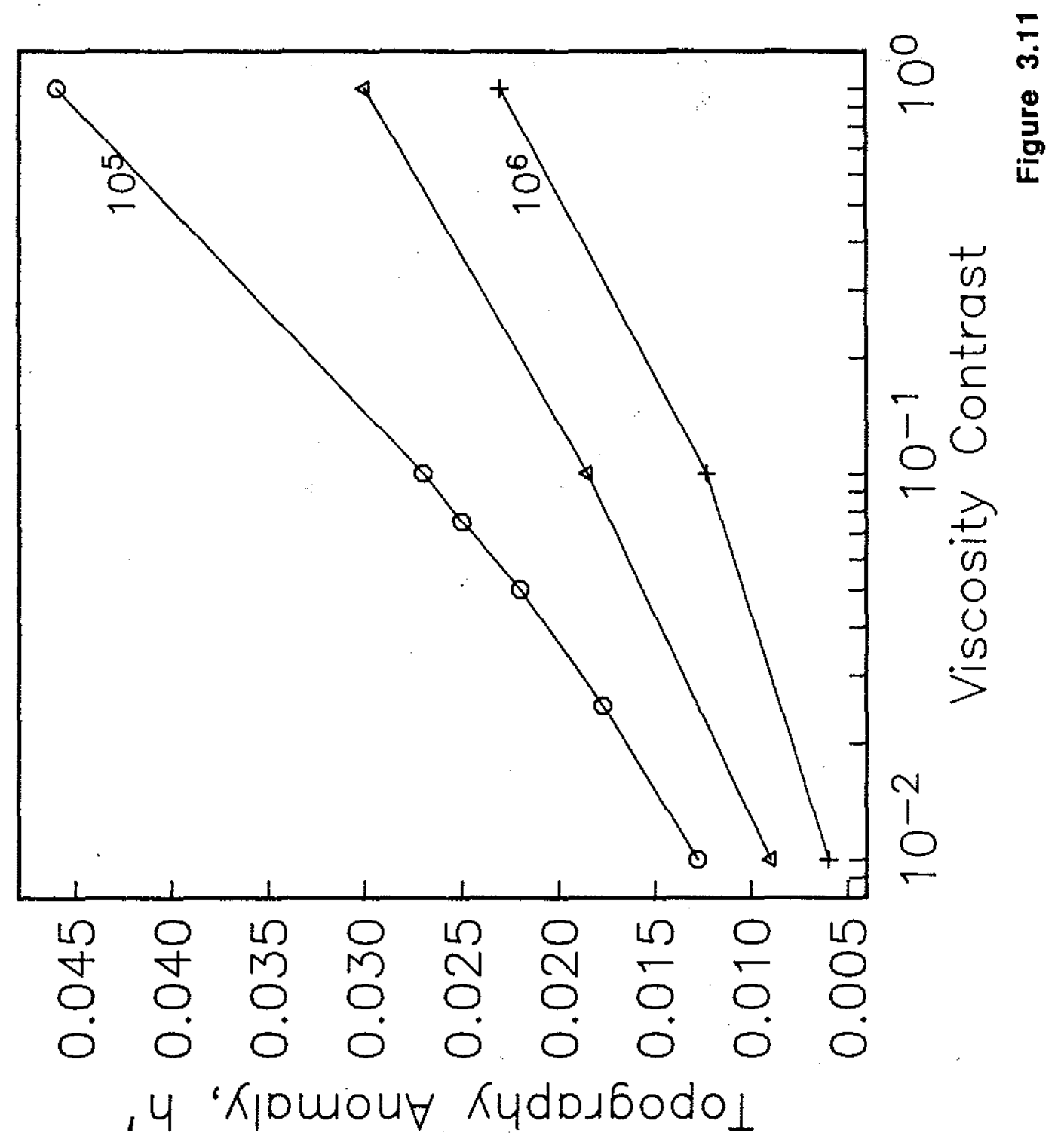




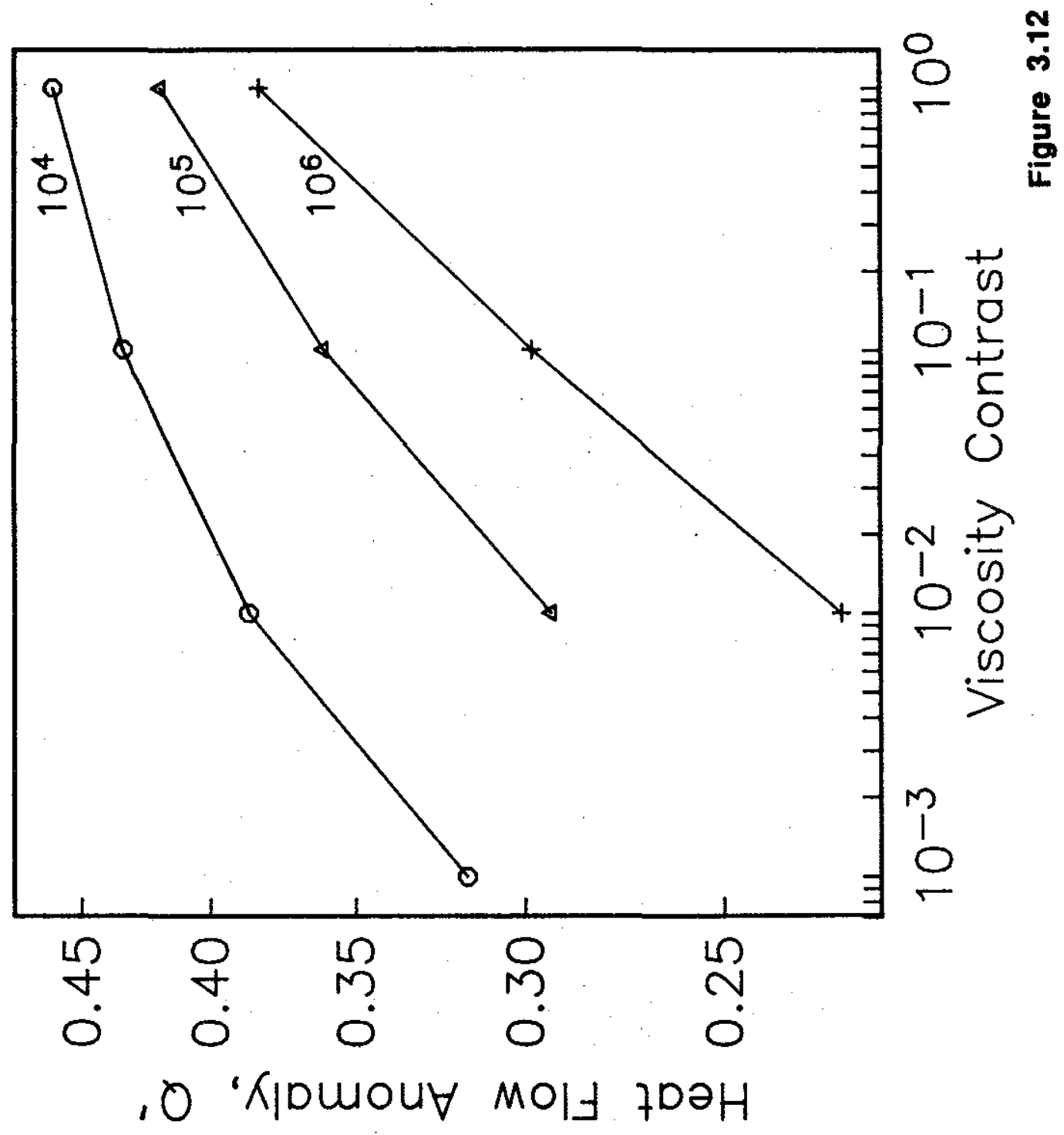




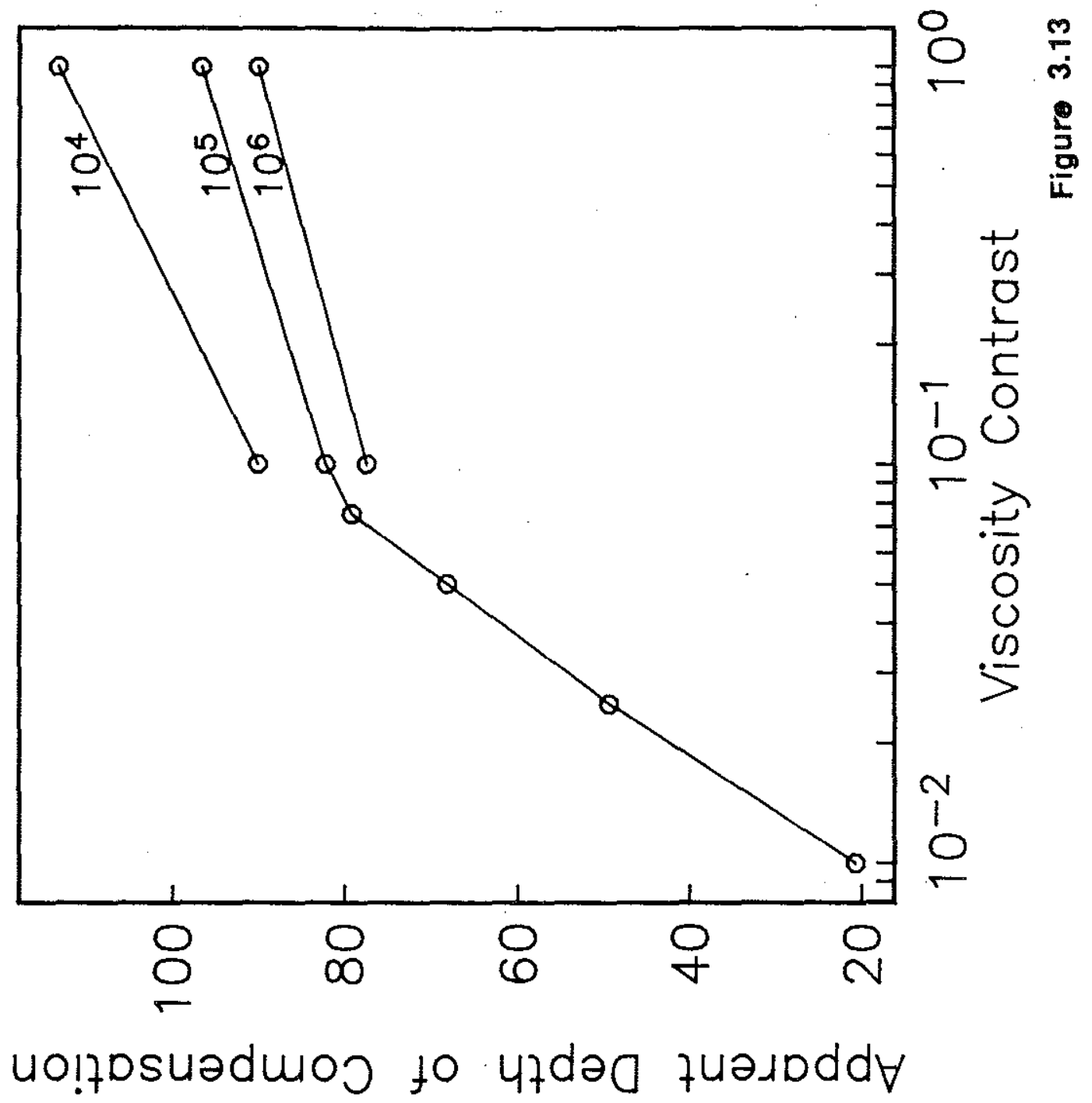




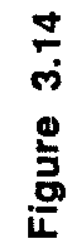

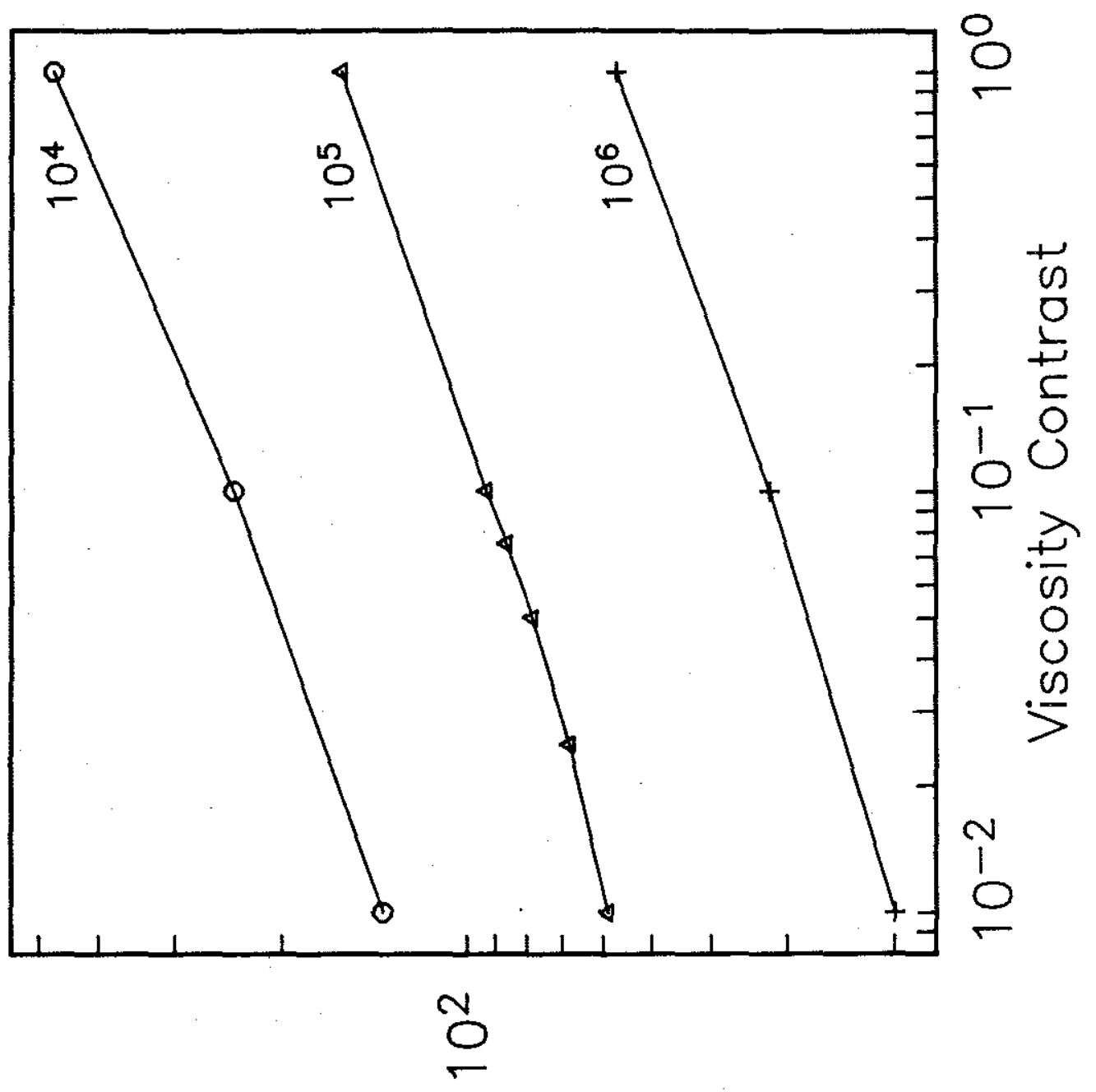

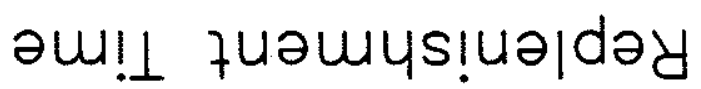


Chapter 4: MANTLE FLOW AND GEOID ANOMALIES AT ERACTURE ZONES

\subsection{Introduction}

Simple conductive cooling cannot explain the depth-age relationships observed for old oceanic plates. In particular, for ages greater than $70 \mathrm{million}$ years, the depth-age curve is shallower than that of a half-space cooling model (Sclater and Francheteau, 1970; Sclater et al., 1971; Parsons and Sclater, 1977). One, somewhat artificial, means of producing the observed deviation from the half-space cooling model is to fix the temperature at a given depth, called the thermal plate thickness (Langseth et al., 1966; McKenzie, 1967; Parsons and Sclater, 1977). This option, which we call the plate model, requires additional heat input into the base of the plate to maintain the higher temperatures within the lithosphere. Crough (1979a) and Heestand and Crough (1981) suggest, however, that the apparent flattening of the depth-age curve is not due to a thermal source which acts everywhere on the base of old lithosphere, but represents the accumulation of thermally rejuvenated lithosphere from hot-spot traces.

Recent global altimeter studies of the oceanic geoid by the GEOS-3 and SEASAT satelites have provided data for another test of the plate model. The plate model predicts a geoid-age relationship which should depart from that of the half-space cooling model near 40 million years (Parsons and Richter, 1980). However, since there are other sources for 
geoid anomalies at wavelengths comparable to plate dimensions which are not related to lithospheric cooling, it is very difficult to separate the geoid-age relationship directly from the global geoid (Detrick, 1981; Cazenave, 1984). Many researchers have tried, therefore, to infer the geoid-age relationship from shorter wavelength geoid anomalies over fracture zones, which are distant from anomalous thermal sources (Crough, 1979b; Detrick, 1981; Sandwell and Schubert, 1982; Cazenave et al., 1982; Driscoll and Parsons, 1987).

Oceanic fracture zones juxtapose oceanic lithosphere with different ages and different thermal structures (Parsons and Sclater, 1977). This abrupt change in the thermal structure results in a rapid change in the height of the geoid and the seafloor depth. For the half-space model, since the geoid height is directly proportional to the age of the lithosphere, assuming the thermal offset between the plate segments remains constant, the geoid step does not decay with time but remains at its initial value (Detrick, 1981; Sandwell and Schubert, 1982). On the other hand, the plate model predicts that, since the lithosphere on either side of the fracture zone decays to the same thermal structure at large ages, the geoid step should decrease with age to zero for very old plates (Detrick, 1981).

Because the Mendocino fracture zone represents a 27 m.y. age offset and because it is isolated from any major hot-spot or site of anomalous crustal structure, it is an 
ideal fracture zone with which to study the geoid slope-age relationship. From geoid profiles obtained by the SEASAT mission, Detrick (1981), Sandwell and Schubert (1982) and Cazenave et al. (1982) estimated geoid offsets across the Mendocino fracture zone. They found that the geoid slopeage relationship decayed with age in accordance with a $100-$ $120 \mathrm{~km}$ thick thermal plate. Furthermore, since the Mendocino fracture zone is far away from any hot spot traces, neither its depth-age nor its geoid slope-age behavior can be explained by the effects of thermal rejuvenation as proposed by Crough (1979) and Heestand and Crough (1981).

However, recent work on the geoid anomalies at fracture zones other than the Mendocino have found behavior that is not explained by the plate model. In particular, Cazenave (1984), in a study of 15 fracture zones including the Udintsev and the Eltanin fracture zones, and Driscoll and Parsons (1987), in a detailed study of the Udintsev and Eltanin fracture zones, have found that the geoid step decays, but not as predicted by a plate model. Driscoll and Parsons (1987) also find that, on profiles that are less than $100 \mathrm{~km}$ apart, the geoid steps can vary radically in magnitude. by up to 0.75 meters. Finally, and perhaps most surprising, is that, at the Falkland-Agulhas fracture zone, Freedman and Parsons (1987) have found that the geoid step reverses in sign. In order to fully understand the geoid anomalies observed over fracture zones, therefore, we must 
understand the effects of other processes than conductive cooling which affect the fracture zone thermal structure.

At short distances from the fracture zone, lateral heat conduction and thermal stresses are important (Sandwell, 1984; Parmentier and Haxby, 1986). These processes alter the appearance of the slope and produce short wavelength features around the fracture zone. Specifically, flexural effects are important in the formation of the "trough" observed on the older side of many fracture zones (Sandwell, 1984). At longer wavelengths, flow beneath the lithosphere plays a dominant role. Since the geoid slope is a long wavelength feature, to understand the geoidslope-age relationship, we must study the flow underneath the fracture zone.

A fracture zone creates a horizontal temperature gradient at the upper boundary of the viscous portion of the upper mantle, and this thermal gradient drives a convective flow. Craig and McKenzie (1986) looked at the effect of such a flow on the geoid and topographic expression of a fracture zone and, in particular, the Mendocino fracture zone. They found that, for an upper mantle viscosity which is consistent with post-glacial rebound values, the geoid signature due to the flow would dominate the observables so that the anomaly due to the change in lithospheric age would not be visible. Since the geoid profiles near the Mendocino fracture zone do not contain this strong convective signal, Craig and Mckenzie postulated the existence of a low 
viscosity zone near the surface which reduces the temperature variations in the convective flow and the magnitude of the resulting geoid anomalies. They presented a model of the uppermost $225 \mathrm{~km}$ of the mantle with a low viscosity channel (150 $\mathrm{km}$ thick) beneath a mechanical plate (75 km thick). This model produced geoid profiles which generally agreed with the observed profiles seen at the Mendocino, demonstrating that models which include the effect of flow underneath a fracture zone can explain the geoid and geoid slope-age behavior seen at the Mendocino fracture zone as well as the thermal plate model. However, Craig and Mckenzie (1982) did not model the flow in the mantle below the low viscosity layer that is induced by the fracture zone flow, nor did they explore the effect of the layer thickness or other parameters on their results. Both of these considerations can strongly affect the theoretical predictions of the geoid anomalies over the fracture zone. Nevertheless, it is clear that a viscosity which is lower than that predicted by the results of post glacial rebound is required in the uppermost oceanic mantle by the geoid anomalies at fracture zones.

The existence of a low viscosity zone which would provide such low viscosities near the base of the thermal plate has been suggested by a number of researchers. Cooper and Kohlstedt (1984) in laboratory experiments on olivine have shown that melt in the triple junction intersections between grains will cause the diffusion path length through 
an aggregate of grains to decrease. The presence of melt in the uppermost mantle would therefore decrease its viscosity and change its deformation behavior. Anderson and Sammis (1970) and Solomon (1972) explain the seismic low velocity zone underneath the plates as a region with a high proportion of melt. Therefore, this low velocity zone most likely reflects a low viscosity zone. Finally, the empirical relationship between viscosity and temperature and pressure predicts a low viscosity zone underneath the plates (Parmentier, 1978; Fleitout and Yuen, 1984a; Buck and Parmentier, 1986). In the thermal boundary layer at the surface, the viscosity will rapidly decrease due to the large temperature gradient with depth. Then, in the adiabatic mantle the viscosity will increase due to the increase in pressure with depth, effectively creating a low viscosity zone underneath the plates.

In this paper, we present a parameter study of the effects of a low viscosity zone on the thermal evolution of fracture zones throughout the upper mantle. To isolate the effect of a low viscosity channel, we have simplified the model to a three layer viscosity structure with a conducting Iid overlying the low viscosity zone which in turn overlies a constant viscosity layer extending to the base of the upper mantle. With this model we have studied the effect on the flow induced in the upper mantle by the fracture zone thermal structure by varying (1) the viscosity in the low viscosity zone, (2) the Rayleigh number based on the full 
layer depth and on the viscosity of the bottom layer, and (3) the low viscosity layer thickness. By isolating these parameters, we can explore the effects of a variable viscosity structure with depth underneath a fracture zone (Parmentier, 1978). After looking at the characteristics of the flow, we calculate the geoid profiles and the geoid slope-age relationships. We compare these results with sample geoid profiles from the Udintsev fracture zone. Finally, we calculate the topographic step versus age relationship from our calculations and compare it with that predicted by the average depth versus age relationships that are observed in the oceanic plates.

\subsection{The Convective Elow}

The numerical model consists of three layers: a low viscosity layer sandwiched between a conducting lid and a constant viscosity layer (see Figure 4.1). The boundary conditions are given by:

$$
\begin{aligned}
\mathrm{w} & =0, \quad \mathrm{z}=0,1, \mathrm{~b} \\
\mathrm{u} & =0, \quad \mathrm{z}=1, \mathrm{~b} \quad \mathrm{x}=0, \mathrm{~h} \\
\sigma_{\mathrm{Xx}} & =0, \quad \mathrm{z}=0 \quad \mathrm{x}=0, \mathrm{~h} \\
\mathrm{~T} & =0, \quad \mathrm{z}=\mathrm{b} \\
\partial \mathrm{T} / \partial \mathrm{x} & =0, \quad \mathrm{x}=0, \mathrm{~h} \\
\partial \mathrm{T} / \partial \mathrm{z} & =0, \quad \mathrm{z}=0 \\
\mathrm{~T} & =1, \quad \mathrm{z}<\mathrm{b}, \quad t=0
\end{aligned}
$$

where $u$ is the horizontal velocity, $w$ is the vertical velocity, $T$ is the tempreature, $t$ is the time, $x$ is the 
horizontal coordinate, $z$ is the vertical coordinate and $\sigma_{x z}$ is the shear stress.

We have nondimensionalized the equations through the following transformations:

$$
\begin{aligned}
\mu^{\prime} & =\mu_{\circ} \mu \\
\left(x^{\prime}, z^{\prime}\right) & =d(x, z) \\
T^{\prime} & =\Delta T T \\
t^{\prime} & =\left(d^{2} / K\right) t \\
\rho^{\prime} & =\rho_{\circ} \rho
\end{aligned}
$$

where the primes denote dimensional quantities and $\mu_{0}$ is the viscosity of the bottom layer, $d$ is the depth of the convecting layer, $\kappa$ is the thermal diffusivity, $\rho_{0}$ is the reference density and $\Delta \mathrm{T}$ is the initial temperature contrast between the bottom and top boundaries. To scale the results we used the values for these parameters as given in Table 4.1 .

The equation of motion, the heat transport equation and the equation of state are then given by lomitting the primes),

$$
\begin{array}{r}
\nabla \cdot \sigma=-\mathrm{R}\left(\mathrm{T}-\mathrm{T}_{\mathrm{O}}\right) \hat{z} \\
\partial \mathrm{T} / \partial \mathrm{t}+\mathrm{u} \cdot \nabla \mathrm{T}=\nabla^{2} \mathrm{~T} \\
1-\alpha \Delta \mathrm{T}\left(\mathrm{T}-\mathrm{T}_{O}\right)=\rho
\end{array}
$$

where $u$ is the velocity vector, $\alpha$ is the thermal expansion coefficient, $\hat{z}$ is a vertical unit vector, $\sigma$ is the stress tensor given by:

$$
\sigma_{i j}=-p \delta_{i j}+\mu\left(\partial u_{i} / \partial x_{j}+\partial u_{j} / \partial x_{i}\right)
$$

where $p$ is the pressure and $R$ is the Rayleigh number: 


$$
R=g_{0} \alpha \Delta \mathrm{Td}^{3} / \mu_{0} \mathrm{k}
$$

where $g_{0}$ is the acceleration of gravity. These equations are solved using a velocity based finite element method with explicit time stepping (Daly and Raefsky, 1985), which has been modified to solve the heat equation with a finite difference method. Although the incompressibility equation:

$$
\nabla . u=0
$$

is never explicitly solved, a penalty function treatment of the pressure forces incompressibility (Hughes et al., 1979). We tested the resolution of each run by repeating the calculation on a larger grid, usually double the size, and comparing the results.

All of the convection calculations began with the same idealized initial temperature structure at $(t=0)$.

$$
\begin{aligned}
& T=0, \quad z=b \\
& T=1, \quad z<b, \quad x<h / 2 \\
& T=\operatorname{erf}\left(z / \kappa \tau_{a}\right), \quad x>h / 2
\end{aligned}
$$

where $\tau_{a}$ is the age offset across the fracture zone. Although large fracture zones are not usually spaced more than $1000 \mathrm{~km}$ apart in the North Pacific for example, we have limited our study to the effects of one age offset for simplicity.

We experimented with a number of box widths. Since the flow migrates away from the fracture zone, however, eventually every box will be too small. For most of our runs, an aspect ratio of 4 to 1 was sufficient but, as soon as the flow was significantly affected by the sidewalls (so 
that the flow was constrained to wavelengths that were integer divisions of the total horizontal length scale in the box), we ended the calculations. Because, at high Rayleigh numbers and viscosity contrasts, the grid must be very dense, some of the resolution calculations were run on a 2 by 1 grid and many of these runs had to be terminated at young ages.

The simplification of the model to three layers involved a number of assumptions about the viscosity structure and thermal source. The first was the approximation of the mechanical portion of the lithosphere by a conducting lid (Jaupart and Parsons, 1985), which was of equal thickness on either side of the fracture zone. Furthermore, since we could not incorporate any growth of the lid with time, its thickness had to be an average of the appropriate thickness for the initial structure and for older ocean floor. As a result the lid is too thick at young ages. To compensate for this shortcoming, we ran the model for a number of lid thicknesses from 0 to $100 \mathrm{~km}$ thick and found that the form of the instabilities and the ensuing flow did not vary appreciably. For much thicker conducting lids which approach the thickness of the fluid layer, the lid increases the aspect ratio of the cells (Craig and McKenzie, 1986). Only the time of the onset of the instability was delayed by a thicker lid. Since this twodimensional calculation cannot hope to give an accurate estimate of the initial time scale of the flow, but only an 
upper bound, we felt that this approximation was justified. We chose a conducting lid thickness of $50 \mathrm{~km}$ for the parameter study which corresponds to a $30-50 \mathrm{~m} \cdot \mathrm{y}$. old plate. In all of these calculations, we concentrated on the flow due to the horizontal temperature gradient at the fracture zone. We neglected other thermal sources such as internal heating or heating from below. Since the upper mantle is believed to have a low concentration of radioactive elements and since we are studying processes which operate on short time scales, we can safely ignore internal heating (McKenzie and Richter, 1981). The omission of heating from below is harder to justify. We ran a number of calculations which included heating from below and found that it did not effect the time scale or initial form of the flow, but that it did increase the vigor of the convection once it was established. In Figure 4.2, we have drawn the temperature anomalies for the same flow model with (Figure $4.2(\mathrm{a})$ ) and without (Eigure $4.2(\mathrm{~b})$ ) heating from below (dT/dz equals a constant of nondimensional unit value). There is a $27 \mathrm{~m} \cdot \mathrm{y}$. Offset across the fracture $\mathrm{zone}$ and there is no viscosity contrast between the layers, the Rayleigh number is $4.5 \times 10^{5}$, and the lid thickness is $75 \mathrm{~km}$. Depicted are the temperature structures at $100 \mathrm{~m} \cdot \mathrm{y}$. and $180 \mathrm{~m} \cdot \mathrm{y}$. (the convective flow becomes appreciable at approximately 80 m.y.). The temperature boundary layer first downwells at approximately the same time in both of these models, but by $150 \mathrm{~m} \cdot \mathrm{y}$. the flow which is heated from below is much more 
vigorous and well developed than the flow without the additional heat flux. The model with heating from below corresponds to the model CMI in Craig and McKenzie (1986) and our results agree very well with theirs.

Since we eventually wanted to compare our results to geoid profiles from the Udintsev fracture zone, we performed the parameter study with an age offset of $15 \mathrm{~m} . \mathrm{y}$.. Because the age offset across the Udintsev is variable from $9-20$ m.y., a $15 \mathrm{~m} . \mathrm{y}$. offset represents an average of the offsets. In Figure $4.2(c)$, we have drawn the flow produced by the model in Figure $4.2(\mathrm{~b})$ with an age offset of $15 \mathrm{~m} \cdot \mathrm{y}$. instead of $27 \mathrm{~m} . \mathrm{y}$.. With the smaller age offset, the flow becomes appreciable $30 \mathrm{~m} \cdot \mathrm{y}$. later, but the development of the flow is very similar. We have shown the temperature anomalies at $130 \mathrm{~m} \cdot \mathrm{y}$. and $210 \mathrm{~m} \cdot \mathrm{y}$. - corresponding to the same time intervals after the flow becomes apparent in the temperature structure as in cases $2(a)$ and $2(b)$. For the larger age offset, where the initial temperature anomaly represents 27 m.y. of conductive cooling, the flow is stronger and the downwellings are fairly uniformly spaced whereas, for the 15 m.y. offset, the flow is weaker and the downwellings are more irregularly spaced. The larger age offset, therefore, drives a stronger, more regular flow which begins earlier. With this viscosity model of the upper mantle consisting of three horizontal fluid layers and driven by a conductive cooling profile with a $15 \mathrm{~m} \cdot \mathrm{y}$. age offset in the center, therefore, we explored the effect on the convective 
flow of (1) low viscosities in the upper layer, (2) the Rayleigh number of the fluid layer, based on the viscosity of the bottom layer, and (3) the relative thicknesses of the two fluid layers. In Table 4.2 are listed the parameters for the runs which we shall discuss.

The first parameter suite varies the Rayleigh number with no viscosity contrast between the fluid layers. In Figure 4.3, we compare the temperature structure at various times for runs with Rayleigh numbers of (a) $10^{6}$ and (b) $10^{7}$. These values bracket most estimates of the Rayleigh number in the upper mantle (McKenzie, 1967; Jarvis and Peltier, 1980; McKenzie et al., 1980). The initial downwelling occurs close to the fracture zone under the older lithosphere, initiating a downward flow. In each of these cases the flow becomes appreciable very quickly at near 20 $\mathrm{m} \cdot \mathrm{y}$. for (a) and $10 \mathrm{~m} \cdot \mathrm{y}$. for (b). We measure the development time through the kinetic energy, the sum over all of the elements in the grid of the mass multiplied by the averaged velocity squared for each element. Although the kinetic energy is a positive definite measure of the onset time, it does not affect the energy balance in this problem and is very small since it is scaled by the inverse of the Prandtl number. However, the kinetic energy exponentially increases as flow begins in the fluid, and we took our definition of the development time to be the time at which this exponential behavior commences. The development times for these runs are delayed by the presence 
of the conducting lid and would also occur earlier in the mantle due to perturbations from other flows, so we consider these values to be upper bounds on the onset time. The downgoing plume is quite thin and near the fracture zone. Downwellings away from the fracture zone do not appear. until much later. At a Rayleigh number of $10^{7}$ (Figure 4.3(b)), the flow consists of very thin downwelling plumes which stretch to the bottom of the box. The first downwelling appears in the temperature structure at close to $10 \mathrm{~m} . \mathrm{y} .$. This downwelling alone destroys the step in the temperature structure very close to the fracture zone, but also introduces temperature anomalies at greater depths. Other downwellings appear close to $30 \mathrm{~m} \cdot \mathrm{y}$. later due to stress and thermal coupling with the initial downwelling and/or simple conductive cooling of the fluid layer.

The development of this flow is different from that seen in small scale convection which is only driven by thermal instabilities in a cooling thermal boundary layer (Foster, 1965a; Foster, 1965b). In that case there is no horizontal temperature gradient to drive the flow, rather the flow is due to internal convective instabilities and can be characterized by the wavelength at which it initially goes unstable. In the case of a fracture zone, a multiplicity of wavelengths are excited, but most of the flow is generated by the initial convection cell and then migrates away from the fracture zone. Simple cooling instabilities can and may occur far from the fracture zone, 
especially at high viscosity contrasts. Since they are less vigorous and are at the same scale as the fracture zone convection cells, they can be easily incorporated into the flow. This study cannot resolve the differences between downwelling plumes that are generated by shear and thermal coupling and those that are instigated by boundary layer instabilities.

In the second series of runs, we held the Rayleigh number constant at $10^{5}$ and the low viscosity layer thickness at $125 \mathrm{~km}$ and varied the viscosity contrast from 1.0 to $10^{-3}$ (Eigure 4.4). As the viscosity contrast increases, the development time of the flow decreases and the characteristic wavelength of the downgoing plume shortens. For a viscosity contrast of one order of magnitude (Figure 4.4(b)), the plume is distorted as it encounters the significant horizontal flow in the low viscosity zone. As the viscosity contrast increases by another order of magnitude (Figure $4.4(c)$ ), the flow initially fills the whole box but then is quickly confined to the low viscosity layer, with the most vigorous flow near the fracture zone. (Near and above a viscosity contrast of $1.3 \times 10^{2}$, the effective Rayleigh number in the low viscosity zone is greater than that for the whole layer, so that it can more easily support convection.) The small scale convection therefore cools the fluid beneath the low viscosity zone near the fracture zone much more effectively. The horizontal temperature gradient near the base of the top 
layer then drives a larger scale flow which extends to the base of the two fluid layers. This larger scale flow eventually dominates the small scale convection, although the signature at the wavelengths due to the small scale flow in the temperature structure persist for a greater length of time. Therefore, even though the low viscosities can initially confine the flow to the top layer, permanent confinement cannot be attained.

In the third parameter suite we varied the Rayleigh number while holding the viscosity contrast at two orders of magnitude and the layer thickness at $125 \mathrm{~km}$ (Figure 4.5 and Figure $4.4(\mathrm{c}))$. The progression of the flow with time in these calculations is very similar to that in the previous series. It begins with a mantle wide flow, followed by a period of confinement of the most vigorous flow to the low viscosity zone. The small scale flow is concentrated near the fracture zone so that cools preferentially underneath it. This cooling leads to a longer wavelength horizontal temperature gradient which reinvigorates convection in the lower layer. This larger scale flow then wipes out the small scale convection. As the overall Rayleigh number increases, the effective Rayleigh number in the top layer also increases, so that the low viscosity zone can more easily concentrate the flow in the uppermost mantle. otherwise varying the Rayleigh number with a fixed viscosity contrast has much the same effect as in the first parameter suite where there was no viscosity contrast: as the Rayleigh 
number increases, the development time and the dominant wavelengths of the flow are shorter, and the downwellings occur closer to the fracture zone.

In the final parameter study, we have varied the thickness of the low viscosity zone while keeping the Rayleigh number at $10^{5}$ and the viscosity contrast at 0.1 (Figure 4.6 and Figure $4.4(\mathrm{~b})$ ). With this suite of calculations, we can specifically address the effect of introducing a second scale length, the depth of the low viscosity zone. When the layer thickness is very thin, near $50 \mathrm{~km}$ (Figure $4.6(\mathrm{a})$ ), the flow resembles that of cooling beneath a conducting lid into a constant viscosity fluid. However, the development time of the flow is much shorter and the next set of convection cells occur much closer to the fracture zone. As the layer thickness increases (Figures $4.4(\mathrm{~b})$ and $4.6(\mathrm{~b}))$, the low viscosity zone can more efficiently concentrate the temperature anomalies. When the low viscosity zone fills half of the box (Eigure 4.6(b)), the downwellings occur in uneven intervals and migrate so that, by $110 \mathrm{~m} \cdot \mathrm{y} .$, the center downwellings have grouped into one strong downwelling.

In summary, these calculations show that the fracture zone thermal structure will generate convection cells which act to erase the initial thermal step. The effect of the flow far from the fracture zone trace depends on the time in which the convection cells are generated away from the thermal step. For a constant viscosity layer underneath a 
conducting lid, this migration time depends on the scale length and vigor of the cells which fill the whole fluid layer. Increasing the Rayleigh number and the viscosity contrast quickens the development of the convection cells and shortens the characteristic wavelength of the downwelling plumes, concentrating their effects near the fracture zone. However, the low viscosity zone also introduces a second time and a second length scale into the flow: the lifetime and the characteristic wavelength of the smaller scale convection respectively. When the Rayleigh number of the low viscosity zone becomes greater than that of the bottom fluid layer, initially the flow will extend through both of the layers, but small scale flow in the low viscosity zone will quickly dominate. Since the small scale convection is concentrated near the fracture zone, it preferentially cools the fluid beneath it, creating a long wavelength horizontal temperature gradient which reinvigorates the flow in the whole layer. Varying the layer thickness causes the secondary scale length and, therefore, the confinement time of the small scale convective flow to vary. The destruction of the thermal step very near the fracture zone, the length and time scales, and the deep cooling will all be apparent in the topography and geoid anomalies calculated for these fracture zone structures. 


\subsection{Calculation of the Geoid and Topography Anomalies}

To calculate the geoid and topography anomalies for the above runs, we follow the Green's function method developed by Parsons and Daly (1983). The temperature field is decomposed into each of its Fourier components and, at each wavenumber, $k$, the kernels, which are the Green's function response of the observables to the temperature field, are calculated. The surface topography kernel represents the effect of a density anomaly at a depth, $z$, on the surface topography, through the transmission of normal stress. The gravity kernel, on the other hand, represents the sum of the gravitational effects of the topography on the boundaries and the density variations in the layer.

The dimensional topography in the Fourier domain, $h(k)$, is then given by:

$$
h^{\prime}(k)=\left[\rho_{O} /\left(\rho_{O}-\rho_{W}\right)\right] \alpha \Delta T d \int_{0}^{b} H(k, z) T(k, z) d z \quad(4.10)
$$

where $H(k, z)$ is the topography kernel. The dimensional gravity, $g(k)$, can be written in the same way:

$$
g^{\prime}(k)=2 \pi G \rho_{\circ} \alpha \Delta T d \int_{l}^{b} G(k, z) T(k, z) d z
$$

where $G(k, z)$ is the gravity kernel and $G$ is the universal Gravitational constant. The gravity kernel can be expressed as the sum of the contributions from the surface topography, the internal density variations and the bottom boundary topography attenuated by the depth of the layer: $G(k, z)=H(k, z)-\exp (-|k| z)+\exp (-|k|) H_{b}(k, z)$ 
where $\mathrm{H}_{\mathrm{b}}(\mathrm{k}, \mathrm{z})$ is the bottom boundary topography kernel. We have calculated the gravity and topography kernels as in Robinson et al. (1987a). Finally the geoid anomaly can be derived from the gravity anomaly with Brun's formula:

$$
N^{\prime}(k)=g^{\prime}(k) / k^{\prime} g_{0}=\left(d / g_{0}\right) g(k) / k
$$

where $N(k)$ is the dimensional geoid in the Eourier domain and $g_{0}$ is the acceleration of gravity.

In Figure 4.7, we have drawn the geoid and topography kernels for the runs in the second parameter suite where the low viscosity layer thickness is $125 \mathrm{~km}$. We have drawn the kernels for a range of wavelengths from the longest wavelength in the box (twice the width of the box) to the wavelength equal to half the depth of the box. As the viscosity in the top layer decreases, the magnitude of both the topography and geoid kernels decreases. Since the low viscosity layer inhibits the transmission of normal stress to the surface, the topography kernels are significantly damped in the low viscosity zone. Because the gravity kernels are the sum of the surface topography kernels with the gravitational response to the internal density distribution and to the bottom topography, the decrease in magnitude of the surface topography kernel allows the negative contribution of the internal density variations to the gravity anomaly to dominate the kernels at depth. The gravity kernels are, therefore, negative at depth for high viscosity contrasts at the shorter wavelengths in Figure 4.7. This negative portion of the gravity kernel 
counteracts the positive effects of the shallower temperature anomalies. Therefore, the magnitude of the geoid anomaly decreases faster than that of the topography as the viscosity contrast increases. In particular, at high viscosity contrasts, the temperature variation due to the small scale convection can subtract from the gravitational effect of the temperature step in the conducting lid, so that the thermal step appears reduced and perhaps even reversed.

Since the mechanical portion of the lithosphere can deform elastically, we explored the effects of an elastic plate on the geoid and topography anomalies. Sandwell and Schubert (1982) have presented a model of flexure at fracture zones and, from a comparison to profiles across the Mendocino fracture zone, they conclude that the plate is elastically continuous across the fracture zone. This elastic behavior in our model was represented by including an additional factor, $v$, in the surface topography kernel (Detrick et al., 1986):

$$
v(k)=\left[1+(k / \beta)^{4}\right]^{-1}
$$

where

$$
\beta^{4}=12\left(1-v^{2}\right) g_{0}\left(\rho_{O}-\rho_{W}\right) / E\left(T_{e}\right)^{3}
$$

and where $T_{e}$ is the elastic plate thickness, $E$ is Young's modulus and $v$ is Poisson's ratio. Values for these parameters are given in Table 4.1. In Figure 4.8, we have drawn the geoid anomalies for runs $1(a)$ and $1(b)$ with and without a $10 \mathrm{~km}$ elastic plate which is a lower bound on the elastic plate thickness for a 30-50 m.y. Old plate (Watts, 
1978). The elastic plate reduces the amplitude of the shorter wavelengths in the geoid. In other calculations, the wavelengths of the convective instabilities which are confined to the low viscosity zone are affected the most by the elastic portion of the plate. Indeed, the geoid signal from a temperature anomaly with a $200 \mathrm{~km}$ dominant wavelength (roughly corresponding to the wavelength of a small scale flow confined to a $100 \mathrm{~km}$ thick low viscosity zone) is reduced by $33 \%$ in the profiles which include the effects of a $10 \mathrm{~km}$ thick elastic plate. Although the elastic plate reduces the slope of the step, it does not affect its overall magnitude which is a longer wavelength signal. Nevertheless, to facilitate a comparison to the Udintsev fracture zone, in the following calculations, we will include the effect of a $10 \mathrm{~km}$ elastic plate.

\subsection{The Geoid Anomaly and the Geoid Slope with Age}

At most fracture zones, the topography is not well known but, since the SEASAT mission, many fracture zones have very good coverage of the geoid over their whole length. We have, therefore, concentrated on the behavior of the geoid anomaly and have calculated the geoid anomalies for all of the runs in the parameter study.

In Figure 4.8, we have drawn the geoid anomalies for runs $1(a)$ and 1 (b) from Table 4.2 at various times. In general, the geoid anomalies are depressed over downwellings and elevated over upwellings and the characteristic 
wavelength of the perturbation to the geoid anomaly directly corresponds to the characteristic wavelength of the flow. In Figure 4.8 (a) (run $1 \mathrm{a}$ ) at $25 \mathrm{~m} \cdot \mathrm{y}$. , the profile still resembles a step modified by horizontal conductive cooling. By $60 \mathrm{~m} . \mathrm{y}$. , the first downwelling has developed in the flow (see Figure 4.3) causing a depression in the geoid on the older side. At $75 \mathrm{~m} \cdot \mathrm{y}$. , the magnitude of this depression is as big as the step itself and, by $90 \mathrm{~m} \cdot \mathrm{y}$. , features caused by the adjacent cells are apparent in the geoid. At 90 m.y., two humps have formed next to the depression, one on the younger side next to the fracture zone and one adjacent to the depression on the older side. They reflect the upwellings on either side of the initial downwelling. In Figure $4.8(\mathrm{~b})$ (run $1 \mathrm{~b}$ ), the geoid profile follows the same general progression as seen in Figure 4.8(a), but much more quickly. By $50 \mathrm{~m} . \mathrm{y} .$, the convective signal dominates the geoid profile with small wavelength (approximately $500 \mathrm{~km}$ ) undulations with amplitudes of over one meter.

In Figure 4.9, we have included a sample fit to the geoid anomaly (from run $2 b$ ) from which we estimate the magnitude of the step. We estimate the geoid and topographic steps by fitting a second order polynomial and a step function to the calculated geoid and topography profiles (Crough, 1979). In order to calculate the geoid step as in Driscoll and Parsons (1987) across the Udintsev fracture zone, $200 \mathrm{~km}$ of the geoid profile to each side of the fracture zone was excluded from the least squares 
fitting procedure. The total length of the profile used was then $1400 \mathrm{~km}$ (where we have also omitted the end effects). Since the flow extends well away from the fracture zone, the estimate of the geoid and topographic step can be quite dependent on the length of the profile that we choose. We do not use the entire length of the geoid and topography anomaly to avoid the effects of the side wall and to mimic the real conditions, but we have run all of the calculations with varying lengths of the profile as a check to see if our preferred $1400 \mathrm{~km}$ profile length has severely biased the results. We calculate the geoid slope as the geoid step divided by the age difference across the fracture zone. In many of the geoid profiles for the cases in this study, the step has disappeared. We only calculate the geoid slope-age relationship, however, using geoid steps from profiles that contain a visible step.

In Figure 4.10, we have drawn the geoid slope-age relationship for these two runs (runs $1 \mathrm{a}$ and $1 \mathrm{~b}$ ). Initially the geoid slope-age relationship behaves like a half-space model but, after the temperature variations due to the flow become appreciable in the fluid layer, it departs from the conductive cooling models. In the case where the Rayleigh number equals $10^{6}$, the step initially decays and then increases, due to the combined effects of the rise and depression near the fracture zone, before finally decreasing. In the case where the Rayleigh number equals $10^{7}$, the step initially decays, but then decreases very 
rapidly, increasing slightly between 40 and $45 \mathrm{~m} . \mathrm{y}$. and becoming negative in portions of the relationship. The geoid slope-age relationship cannot therefore be characterized by a simple model; rather its behavior is quite complicated. However, in both of these cases without a low viscosity zone, after the initial downwelling becomes appreciable the magnitude of the step at first increases reflecting the perturbation in the geoid anomaly very near the step. As the convection cell develops, the older side of the fracture zone initiates a downwelling which depresses the geoid whereas the other side of the fracture zone experiences an upwelling elevating the geoid. These effects combined cause a rapid increase in the apparent step height in run $1(a)$. When there is an appreciable viscosity contrast at depth, however, this long wavelength effect is minimized.

As we vary the viscosity contrast through runs 2 (a) 2 (c) (from Table 4.2) the characteristic wavelength and magnitude of the convective perturbations to the geoid anomalies decrease (Figure 4.11). For the case of a constant viscosity fluid at a Rayleigh number of $10^{5}$ (run 2a), the geoid signal from the flow dominates the anomaly and swamps the step signature. At a viscosity contrast of one order of magnitude (run $2 \mathrm{~b}$ ), although the convective signal eventually swamps the step signature until ages over $100 \mathrm{~m} . \mathrm{y} .$, the step signature remains visible. By a viscosity contrast of two orders of magnitude (run 2c), 
however, the magnitude of the convective perturbations to the geoid are much less than the step signature and they are of relatively short wavelength. Because the kernels become negative in the low viscosity zone at this viscosity contrast, the enhanced cooling underneath the fracture-zone on the older side counteracts the effects of the shallower conductive cooling and the direction of the step is reversed. In Figure 4.12, we have drawn the geoid slope-age behavior for these runs. For run 2(a), the step does not decay and is eventually dominated by the convective signal. At the intermediate viscosity contrast (run 2b), the step does decay somewhat, before the convective signal takes over and the estimated step increases. For run 2(c) (Figure $4.11(\mathrm{c}))$, however, the step signal quickly decreases and eventually reverses sign. The profiles containing the step reversal are similar to profiles seen at the Falkland-Agulas fracture zone (Freedman and Parsons, 1987).

As we vary the Rayleigh number for a viscosity structure with a viscosity contrast at $175 \mathrm{~km}$ depth of two orders of magnitude (runs 3 and $2 \mathrm{c}$ in Table 4.2, see Figure 4.11 and 4.13), we find very similar behavior as in runs $2(a)-2$ (c) (Figure 4.11) where we increased the viscosity contrast. The characteristic wavelength of the geoid anomaly and the departure time from the half-space cooling model decreases, and the step signature remains for longer times before being destroyed by the convective signal. In 
Figure 4.14, we have drawn the geoid slope-age relationships for these runs.

When we varied the thickness of the low viscosity zone (runs $4 \mathrm{a}, 2 \mathrm{~b}$ and $4 \mathrm{~b}$ in Table 4.2 ), we found that the thin layers $(50 \mathrm{~km}$ and $125 \mathrm{~km})$ produced very similar geoid anomalies (Figure 4.15) and geoid slope-age behavior (Figure 4.16). When the low viscosity zone fills half of the box, however, the topography and geoid profiles change in character and the step signature disappears more quickly, because the longer scale length for the flow in the low viscosity zone causes the geoid perturbations to extend to greater distances from the step, destroying the geoid slopeage relationship.

From the previous discussion, it is evident that the onset and magnitude of the downwellings depend on the viscosity of the fluid in the cooling boundary layer. We, therefore, assign to each of our runs an "effective Rayleigh number" which is based on the full depth of the fluid layer but on the viscosity of the top layer. (Note: the Rayleigh number for the top layer should more properly be defined with the depth of the top layer but, to compare runs with different low viscosity zone thicknesses, we have used the depth of the total fluid layer. We also do not mean to suggest that this effective Rayleigh number can be rigorously related to the Nusselt number or any other parameter of the flow.) For the runs at an effective Rayleigh number of $10^{5}$, the geoid slope-age relationship 
initially remains constant until the convective temperature variations become appreciable, swamping the geoid step, and the geoid slope-age relationship increases. At an effective Rayleigh number of $10^{6}$, the geoid step initially decreases slightly until the initially downwelling in the temperature structure commences at 40-80 m.y., distorting the geoid step. At an effective Rayleigh number of $10^{7}$, the first temperature downwelling occurs at $10 \mathrm{~m} . \mathrm{y}$. with a magnitude small compared to the initial thermal step and the geoid step decays very rapidly.

\subsection{Comparison to the Udintsev Eracture zone}

In Figure 4.17, we have drawn geoid profiles from the east and west sides of the Udintsev fracture zone obtained from SEASAT altimetry, with the GEM9 field removed, up to and including degrees of order $1=m=10$, and the regional field removed (Driscoll and Parsons, 1987). These profiles show a clear step across the fracture zone which decays with age but, at wavelengths of $300-600 \mathrm{~km}$, the profiles are in general highly variable.

In Figure 4.18, we show the geoid slopes estimated from the profiles in Figure 4.17 and others over the Udintsev fracture zone (Driscoll and Parsons, 1987). The geoid step does decay, but not in accordance with a thermal plate model. Perhaps the most striking feature is the variability in the geoid slope over short time scales. Geoid profiles less than $200 \mathrm{~km}$ from each other can have steps which differ 
in size by as much as $75 \mathrm{~cm}$. However, the general trend of the data on both sides of the fracture zone is to decay initially but then to level off with some long wavelength fluctuation with age.

In Figure 4.19, we have drawn the geoid profiles predicted by run 5 in Table 4.2 . The viscosity contrast in this run is 0.1 , the Rayleigh number is $3.2 \times 10^{5}$ (giving $n$ effective Rayleigh number of $3.2 \times 10^{6}$ ), the conducting lid thickness is $50 \mathrm{~km}$ and the layer thickness is $125 \mathrm{~km}$. Since the precise shape of the geoid profile depends on the deformation properties of the plate, we are hesitant to produce a model which attempts to fit the short wavelength data exactly. In particular, most of the smaller wavelength features in the data near the fracture zone can be explained as a result of the flexure and thermal stresses at the fracture zone (Sandwel1, 1984; Parmentier and Haxby, 1986). However, the theoretical profiles are in general agreement with the longer wavelength anomalies, at which convective flow has a dominant effect. The geoid step is clearly visible out to ages greater than $50 \mathrm{~m} . \mathrm{y}$. and, as in the observed profiles, the effects of the convection at depth are not marked in the theoretical geoid profiles. In Figure 4.20, we have drawn the geoid slope-age relationship from this run. The geoid slope initially decays with age, but after $40 \mathrm{~m} . \mathrm{y}$. it rises again due to the increasing geoid low on the older side. This variation is similar in character to the geoid slope-age relationship on both sides of the 
Udintsev fracture zone, but is perhaps most like that on the eastern side.

The geoid anomalies and geoid-slope behaviour of run 5 (Eigures 4.19 and 4.20 ) which reproduce the geoid steps and geoid slope-age relationship at the Udintsev are typical of the runs in this study with an effective Rayleigh number of $10^{6}$ or above, excepting those cases at an effective Rayleigh number of $10^{7}$ where the step reverses. At the Udintsev fracture zone, no step reversals are seen, but at the Falkland-Agulas fracture zone step reversals are evident (Freedman and Parsons, 1987) and perhaps a model with a higher effective Rayleigh number is required. The remaining question is whether this set of convective models, with effective Rayleigh numbers above $10^{6}$, can also explain the depth-age data for the oceanic plates.

\subsection{Depth Versus Age}

With the assumption of isostatic compensation, the geoid anomaly is a vertical integral over the depth of the density structure weighted inversely with the depth. The topography anomaly is the same integral, but not weighted with depth so that it is less sensitive to the deep convective temperature anomalies. Rather the topography anomaly is predominantly affected by the temperature structure in the conducting lid, which is controlled by conduction. Furthermore, as the viscosity contrast decreases, the response of the topography anomaly to the 
convective anomalies at depth below the fracture zone decreases (see Figure 4.7). In fact, the topographic kernels are near zero under the low viscosity zone at a viscosity contrast of two orders of magnitude.

Since the topographic step is a long wavelength feature, it must satisfy the average depth versus age relationship that is observed in the oceans. In Figure 4.21, we have drawn the topographic step with age for a fracture zone, with a $15 \mathrm{~m} \cdot \mathrm{y}$. offset, that is predicted by the best fitting curves to the average depth versus age data in the North Pacific (Parsons and Sclater, 1977). Along with this theoretical curve, we have drawn the topographic step-age relationships for four calculations: (1) no viscosity contrast with a Rayleigh number of $10^{6}$ (run 1a); (2) no viscosity contrast at a Rayleigh number of $10^{7}$ (run 1b); (3) two orders of magnitude viscosity contrast with a Rayleigh number of $10^{5}$ (run 2C); and (4) an order of magnitude viscosity contrast with a Rayleigh number of $3.2 \times 10^{5}$ (run 5 ).

When there is no viscosity contrast, the longer wavelength topography anomalies, corresponding to convection throughout the box, quickly destroy the topographic step and replace the conductive step signature with a convective anomaly. As the viscosity contrast increases, the convective wavelengths shorten and decrease in magnitude, so that they do not obscure the topographic step. In these cases, the topographic step follows the predicted step from 
the average depth versus age relationships closely. Eventually, as seen in the convection calculations, the convective flow will fill the whole box and the longer wavelength temperature anomalies will destroy the appearance of the step. The low viscosity zone, however, delays the appearance of this longer wavelength convection so that it becomes appreciable only when the topographic step is already small. These runs correspond to effective Rayleigh numbers of (1) $10^{6} ;$ (2) $10^{7} ;$ (3) $3.2 \times 10^{6} ;$ and (4) $10^{7}$. The effective Rayleigh number, therefore, does not govern the behavior of the step. Rather, at these Rayleigh numbers, a viscosity contrast is required at a shallow depth in the upper mantle to shorten the wavelength and decrease the magnitude of the convective anomalies, and to downweight the effect of the temperature anomalies at depth through the kernels.

In summary, if flow under fracture zones is an important phenomenon in the Earth's mantle, then it is important that some mechanism, such as that exerted by a low viscosity zone on the flow, be present to diminish the effect of longer wavelength temperature anomalies on the topography anomalies. The low viscosity zone decreases both the wavelength and magnitude of the temperature anomalies. It also concentrates the response of the topography anomaly to shallower depths and, for a viscosity contrast of two orders of magnitude, the kernels are effectively zero beneath the low viscosity zone. In particular, run 5 which 
produces geoid anomalies and a geoid slope-age relationship that are in general agreement with the observed geoid anomalies at the Udintsev fracture zone also produces a depth-age relationship which is consistent with the observed depth-age relationships.

\subsection{Discussion}

Subject to the assumptions made in this study, some further conclusions can be drawn about the effect of a low viscosity zone on the flow at fracture zones and the calculation of its surface observables. First, the temperature anomalies at depths below the low viscosity zone very important in the calculation of the surface observables, but perhaps less so than if the low viscosity zone did not exist in the case of topography. In the case of constant viscosity convection, the topography and geoid kernels are appreciable down to great depths (see Figure 4.7). As the viscosity in the top layer decreases, the topography kernels decrease in magnitude at depth below the low viscosity zone. The geoid kernels are more complicated and change sign at depth. To accurately calculate the geoid and topography anomalies for a convective system, therefore, one must include the temperature anomalies down to the bottom of the convective layer.

Second, although the convective flow at a fracture zone, with a very high viscosity contrast in the viscosity structure, will initially prefer the low viscosity zone, the 
flow will never be confined to it. Because of the presence of the initial temperature gradient, the flow near the fracture zone will have a greater amplitude that the flow farther away from the fracture zone. This flow preferentially cools underneath the fracture zone and instigates convection throughout the fluid layer, and the longer wavelength flow will eventually dominate the fluid flow. The low viscosity zone, therefore, only delays the instigation of appreciable longer wavelength convective flow. Craig and Mckenzie (1986) did not include the effects of the temperature anomalies at depths below $225 \mathrm{~km}$ in the upper mantle for their models representing a low viscosity zone. They artificially placed a boundary at $225 \mathrm{~km}$ depth, which constrains the convective flow to shorter wavelengths and bars the effects of cooling at greater depths. Einally, in the Earth, the average topography data supports the simple plate cooling models. The geoid anomalies at fracture zones do not in many cases. It has been puzzling that these two observables predict different conclusions since, in constant viscosity convection, they operate in tandem. However, with the addition of a low viscosity zone, the topography and geoid anomalies behave very differently at these short wavelength. In particular, in the above examples, with convection beginning at $50 \mathrm{~km}$ in depth, the assumptions of the plate model are violated, yet the observed depth-age relationship which follows the plate model can be satisfied. This model is further evidence that 
the plate model description of the depth-age relationship is not unique.

There are three major assumptions inherent in our model which could strongly effect the above conclusions. First, we approximate the viscosity structure with only three layers of constant viscosity and neglect the effects of temperature and pressure on the viscosity. This assumption imposes a depth scale into the fluid layer, corresponding to the depth of the low viscosity zone, which remains throughout the calculation and does not vary with time as when the effects of temperature and pressure are included. This depth scale controls the wavelength of the small scale convection which is in turn reflected in the observables. It does not, however, affect our conclusions that the low viscosity zone induces small scale convection, that significant cooling occurs below the low viscosity zone, and that the low viscosities downweight the effect of the temperature anomalies at depth on the surface topography. Second, we assume that the fluid in the two dimensional plane of the model remains in that plane and that advection perpendicular to the plane is negligible. We, therefore, ignore the effects of a shear flow perpendicular to the plane associated with the motion of the conducting lid. This shear flow would primarily affect the time scales of the onset of the different wavelength flows and, in particular, the onset of the longest wavelength flows, corresponding to twice the depth of the fluid layer. These flows would 
occur earlier due to shear and thermal coupling in three dimensions and might, in fact, occur so early that they eliminate the period of confinement to the low viscosity zone. However, the effect of shear and thermal coupling may be diminished by the low viscosity zone and by the direction of relative motion which, since fracture zones are often not aligned in the direction of motion of the plate, might not be directly perpendicular to the plane of the calculation. Nevertheless, the effect of advection perpendicular to the plane of our calculations could affect the relative timing of the stages in the convective flow, especially as regards the confinement of the flow to the low viscosity zone. Finally, we constrain the fluid to circulate in a region corresponding to only the upper mantle. This assumption limits the longer wavelength flow to wavelengths near twice the depth of the box or $1200 \mathrm{~km}$, in our calculations. Since this wavelength is close to the mean length of the observed SEASAT geoid and topography profiles over fracture zones, it has a strong effect on the predicted depth-age and geoid slope-age relationships. If our model instead included the whole of the mantle and the $670 \mathrm{~km}$ discontinuity was not a significant fluid boundary, then this wavelength of convection would not be so pronounced in the observables. However, the low viscosity zone would still be required to dampen the effects of temperature variations beneath the plates in order to satisfy the depthage relationship, and to introduce the time dependence and 
small scale variability seen in the geoid slope-age relationship.

\subsection{Conclusions}

With a finite element numerical method, we have examined the flow in the upper mantle driven by a fracture zone thermal structure. Our model assumes a simple three layer viscosity structure consisting of a rigid conducting lid overlying a low viscosity layer which in turn overlies a unit viscosity layer extending to the base of the upper mantle (Figure 4.1). We have studied the effects of varying the viscosity in the low viscosity zone, the Rayleigh number based on the viscosity of the lower layer and the thickness of the low viscosity layer, on the flow and on the topographic and geoid expression of the fracture zone.

The flow initially acts to decrease the thermal step, and the first convection cell grows immediately beneath the conducting lid at the fracture zone, advecting hotter material from the younger side of the fracture zone to the colder side. Other cells grow later through shear and thermal coupling, and from boundary layer instabilities away from the fracture zone. In general, the flow is very time dependent and becomes more so as the Rayleigh number and viscosity contrast of the fluid layers are increased. Also, as they increase, the characteristic wavelengths and temperature variations in the convection cells decrease. 
We have found that the flow is primarily dependent on the viscosity of the top layer where the temperature gradients are initially the strongest, and we have defined an effective Rayleigh number for our calculations which is based on the viscosity of the top layer and the depth of the whole fluid layer (the top and bottom layers together). When this Rayleigh number is less than $10^{6}$, the convective anomalies dominate both the topographic and geoid observables and, after very young ages, the step signature of the conductively cooling thermal plates is no longer visible in the topography and geoid anomalies. Above an effective Rayleigh number of $10^{6}$, however, the convection anomalies are smaller in wavelength and amplitude and the step signature remains visible out to larger ages. Rayleigh numbers of $10^{6}$ to $10^{7}$ and low viscosity zones of one to two orders of magnitude of viscosity lower than the rest of the upper mantle have been estimated for the upper mantle, so that an effective Rayleigh number (based on the viscosity of the top layer and the depth of the top and bottom layers combined) of $10^{6}$ or greater is not unrealistic for the uppermost mantle.

Since the convective flow acts to decrease the thermal step across the fracture zone, the geoid and topography relationships with age are different than would be produced. by conductive cooling of the plates alone. In general the geoid slope-age relationship is time dependent and nonmonotonic, and can even produce step reversals. This 
behavior has been observed at a number of fracture zones, including the Udintsev, the Eltanin, the Falkland-Agulhas and the Amsterdam fracture zones (Cazenave et al., 1982; Driscoll and Parsons, 1987; Freedman and Parsons, 1987). The topographic anomaly, on the other hand, follows the theoretical curve produced by the average depth-age relationships in the oceans, but only if there exists a low viscosity zone in the model. Otherwise, the convective flow extending throughout the upper mantle destroys the appearance of the topographic step.

Craig and Mckenzie (1986) found that a fluid model of a $150 \mathrm{~km}$ thick low viscosity zone under a conducting lid of 75 $\mathrm{km}$ with a viscosity that is two orders of magnitude below the post-glacial rebound value can reproduce the general characteristics of the geoid profiles over the Mendocino fracture zone. If this model were posed in the geometry of our model, it would have a Rayleigh number of $4.5 \times 10^{7}$. Their model differs from most of those presented in this paper since it represents a $27 \mathrm{~m} . y$. offset and includes heating from below, so that the predicted flow would be more vigorous. Nevertheless, the basic effect of a low viscosity zone on the geoid profiles at the fracture zone are illustrated by their model, and the effective Rayleigh number which they present for the Mendocino fracture zone is in the range of models that we present for the Udintsev. Since they do not include the effects of cooling below the low viscosity zone, however, their calculations of the geoid 
are not fully correct and this is illustrated in that their model does not predict step reversals seen in our model at effective Rayleigh numbers of $10^{7}$ or above.

Through the paramter study, we have found that a range of models reproduce the data at the Udintsev fracture zone. From the geoid data, the thickness of the low viscosity zone is not constrained, except where step reversals are observed. In fact, a model with a viscosity that is one or more orders of magnitude below the post-glacial rebound value throughout the mantle would produce reasonable geoid profiles. However, the topography step-age data requires a low viscosity zone which 100-300 km thick. Furthermore, since it is the viscosity of the top layer which controls the character of the flow (as reflected in the effective Rayleigh number), the exact value of the viscosity contrast and the Rayleigh number are not well constrained. A viscosity contrast of at least $0.1-0.01$ is required, however, to dampen and switch the sign of the kernels at depth in and below the low viscosity zone. Therefore, models can fit the data with a low viscosity zone which is 100-300 $\mathrm{km}$ thick, a viscosity contrast which is greater than one order of magnitude and a Rayleigh number which is at least $10^{5}$.

We have presented in detail the geoid anomalies, geoid slope-age and depth-age results of one calculation (run 5, Table 4.2) which is an example of the set of runs with an effective Rayleigh number of $10^{6}$ or over. The viscosity 
contrast is 0.1 , the Rayleigh number is $3.2 \times 10^{5}$, (the layer thickness is $125 \mathrm{~km}$ and the conducting lid thickness is 50 $\mathrm{km})$, so that its effective Rayleigh number is $3.2 \times 10^{6}$. This run produces the long wavelength features of the observed geoid anomalies at the Udintsev (Figures 4.17 and 4.19), and it also predicts the geoid slope-age relationship and the depth-age relationship (Figure 4.18,4.20 and 4.21). This run is typical of the calculations with an effective Rayleigh number of $10^{6}$ or higher, effective for runs at or above an effective Rayleigh number of $10^{7}$ where the step reverses.

In conclusion, the geoid and topography profiles over a fracture zone are very sensitive to flow beneath it and to the exact viscosity structure of the uppermost mantle. This flow is time dependent and involves a multiplicity of length scales. When the Rayleigh number based on the full layer depth and the viscosity of the top layer is greater than $10^{6}$, a convective model can explain the geoid slope-age behavior that is observed at the Udintsev fracture zone. However, a low viscosity zone in the uppermost part of the mantle is required to produce the long wavelength depth-age relationship observed in the oceans.

\section{Acknowledgments}

We are grateful to Stephan Daly and Arthur Raefsky for the use of their finite element code and to steve Daly for his advice and encouragement. We would also like to thank 
Claire Craig, Marc Parmentier, Marcia McNutt, Joe Pedlosky, Tom Jordan, Adam Freedman, Dan McKenzie and Karen Fischer for helpful discussions. This work has been supported by NASA Geodynamics program grant NAG5-415, and National Science Foundation grant EAR-8306249. We would also like to thank Tom Jordan for making computing time on the Alliant EX/1 available to us. 
Table 4.1

\begin{tabular}{|c|c|c|}
\hline Variable & Description & Value \\
\hline d & depth scale & $600 \mathrm{~km}$ \\
\hline$\rho_{\circ}$ & $\begin{array}{l}\text { average mantle } \\
\text { density }\end{array}$ & $3330 \mathrm{~kg} / \mathrm{m}^{3}$ \\
\hline$P_{W}$ & density of water & $1025 \mathrm{~kg} / \mathrm{m}^{3}$ \\
\hline go & $\begin{array}{l}\text { surface gravitational } \\
\text { acceleration }\end{array}$ & $9.82 \mathrm{~m} / \mathrm{s}^{2}$ \\
\hline $\mathbf{K}$ & $\begin{array}{l}\text { average mantle thermal } \\
\text { diffusivity }\end{array}$ & $10^{-6} \mathrm{~m}^{2} / \mathrm{s}$ \\
\hline$\alpha$ & $\begin{array}{l}\text { average mantle thermal } \\
\text { expansion coefficient }\end{array}$ & $3.1 \times 10^{-5} \circ^{\circ} \mathrm{C}^{-1}$ \\
\hline $\mathrm{T}_{\mathrm{e}}$ & elastic plate thickness & $10 \mathrm{~km}$ \\
\hline $\mathbf{v}$ & Poisson's ratio & 0.25 \\
\hline$\Delta \mathrm{T}$ & $\begin{array}{l}\text { Temperature contrast } \\
\text { across the box }\end{array}$ & $1365^{\circ} \mathrm{C}$ \\
\hline $\mathrm{E}$ & Young's Modulus & $8 \times 10^{10} \mathrm{~N} / \mathrm{m}^{2}$ \\
\hline
\end{tabular}


Table 4.2

Run Rayleigh Viscosity Layer Grid Figure

- Number Contrast Thickness (elems) Number\#

\begin{tabular}{|c|c|c|c|c|c|}
\hline $\begin{array}{l}i \\
i i \\
i i\end{array}$ & $\begin{array}{l}4.2 \times 10^{5} \\
4.2 \times 10^{5} \\
4.2 \times 10^{5}\end{array}$ & $\begin{array}{l}1.0 \\
1.0 \\
1.0\end{array}$ & $\begin{array}{l}\text { n.l. } \\
\text { n.l. } \\
\text { n.l. }\end{array}$ & $\begin{array}{l}192 \times 27^{1} \\
192 \times 27^{2} \\
192 \times 27^{3}\end{array}$ & $\begin{array}{l}4.2 \mathrm{a} \\
4.2 \mathrm{~b} \\
4.2 \mathrm{c}\end{array}$ \\
\hline $\begin{array}{l}1 \mathrm{a} \\
1 \mathrm{~b}\end{array}$ & $\begin{array}{l}106 \\
10^{7}\end{array}$ & $\begin{array}{l}1.0 \\
1.0\end{array}$ & $\begin{array}{l}\text { n.l. } \\
\text { n.1. }\end{array}$ & $\begin{array}{l}200 \times 50 \\
200 \times 50\end{array}$ & $\begin{array}{l}4.3,4.8,4.10,4.21 \\
4.3,4.8,4.10,4.21\end{array}$ \\
\hline $\begin{array}{l}2 a \\
2 b \\
2 c\end{array}$ & $\begin{array}{l}105 \\
10^{5} \\
10^{5}\end{array}$ & $\begin{array}{l}1.0 \\
0.1 \\
0.01\end{array}$ & $\begin{array}{l}\mathrm{n} .1 \\
125 \mathrm{~km} \\
125 \mathrm{~km}\end{array}$ & $\begin{array}{l}140 \times 38 \\
200 \times 50^{*} \\
200 \times 50^{*}\end{array}$ & $\begin{array}{l}4.4,4.11,4.12 \\
4.4,4.11,4.12 \\
4.4,4.11,4.12,4.21\end{array}$ \\
\hline 3 & $10^{4}$ & 0.01 & $125 \mathrm{~km}$ & $200 \times 50$ & $4.5,4.13,4.14$ \\
\hline $4 a$ & $\begin{array}{l}10^{5} \\
10^{5}\end{array}$ & $\begin{array}{l}0.1 \\
0.1\end{array}$ & $\begin{array}{rl}50 & \mathrm{~km} \\
300 \mathrm{~km}\end{array}$ & $\begin{array}{l}140 \times 38 \\
200 \times 50\end{array}$ & $\begin{array}{l}4.6,4.15,4.16 \\
4.6,4.15,4.16\end{array}$ \\
\hline & $3.2 \times 10^{5}$ & 0.1 & $125 \mathrm{~km}$ & $200 \times 50^{*}$ & $4.19,4.20,4.21$ \\
\hline
\end{tabular}

n.1. - no low viscosity layer

$1-8 \times 1$ box, with a $27 \mathrm{~m} . y$. offset across the fracture zone and a conducting lid thickness of $75 \mathrm{~km}$

2 - as for 1 and with heating from below ( 1 H.F.U.)

$3-8 \times 1$ box, with a $15 \mathrm{~m} . \mathrm{y}$. offset across the fracture zone and a conducting lid thickness of $75 \mathrm{~km}$

* - tested on a $146 \times 79(2 \times 1)$ grid for accuracy 


\section{Eigure Captions}

Figure 4.1: Geometry and boundary conditions of the model where "a" is the thickness of the bottom layer, "b" is the thickness of the whole box, "c" is the prescribed flux and " $h$ " is the width of the box.

Figure 4.2: Temperature contours of the runs discussed in the text with $R a=4.2 \times 10^{5}$, no viscosity contrast at depth, a conductive lid of $75 \mathrm{~km}$ (with tick marks marking its lower boundary), and an $8 \times 1$ box with $192 \times 27$ elements. (a) has a 27 m.y. age offset and heating from below (run i); (b) has a 27 m.y. age offset but no heating from below (run ii); and (c) has a $15 \mathrm{~m} \cdot \mathrm{y}$. age offset and no heating from below (run iii). The temperature contours represent $136^{\circ} \mathrm{C}$ increments.

Fiqure 4.3: Temperature contours for runs with no viscosity contrast: (a) with a Rayleigh number of $10^{6}$ (run 1 ); and (b) with a Rayleigh number of $10^{7}$ (run 1b) from Table 4.2. The temperature contours represent $90^{\circ} \mathrm{C}$ increments and the ticks mark the lower boundary of the conducting lid.

Figure 4.4: Plots of the temperatures and streamines for runs with a Rayleigh number of $10^{5}$, a low viscosity zone thickness of $125 \mathrm{~km}$ : (a) with no viscosity contrast (run 2a); (b) with an order of magnitude viscosity contrast (run 2b); and (c) with a viscosity contrast of two orders of 
magnitude (run 2c), from Table 4.2. The temperature contours represent $90{ }^{\circ} \mathrm{C}$ increments except for (c) where they represent only $75^{\circ} \mathrm{C}$, and the ticks mark the boundaries of the low viscosity zone.

Eigure 4.5: Plots of the temperatures for run 3 with a Rayleigh number of $10^{4}$, a viscosity contrast of two orders of magnitude and a top layer thickness of $125 \mathrm{~km}$ from Table 4.2. The temperature contours represent $90^{\circ} \mathrm{C}$ increments, and the ticks mark the boundaries of the low viscosity zone.

Eigure 4.6: Plots of the temperatures for runs with a Rayleigh number of $10^{5}$, a viscosity contrast of one oder of magnitude: (a) a top layer thickness of $50 \mathrm{~km}$ (run $4 \mathrm{a}$ ); and (b) a top layer thickness of $300 \mathrm{~km}$ (run $4 \mathrm{~b}$ ) from Table 4.2. The temperature contours represent $136^{\circ} \mathrm{C}$ increments, and the ticks mark the boundaries of the low viscosity zone.

Eigure 4.7: The topography and gravity kernels for wavelengths: 8d-solid line, 2d-long dash, d-medium dash, and $(d / 2)$-short dash, for a top layer thickness of $125 \mathrm{~km}$ and a viscosity contrast of: (a) 1.0 ; (b) 0.1 ; (c) 0.01 .

Figure 4.8: The geoid profiles at various times for runs: (a) 1 (a); and (b) 1 (b), from Table 4.2 as in Figure 4.3. The geoid is in meters and the distance in kilometers. The 
dashed line is without an elastic plate and the solid line includes a $10 \mathrm{~km}$ elastic plate.

Figure 4.9: A sample fit to the geoid versus distance plot (from run 2b) from which we estimate the magnitude of the step.

Figure 4.10: The geoid slope versus age relationships for runs $1(a)$ and 1 (b) (with a Rayleigh number of $10^{6}$ and $10^{7}$, respectively, and no viscosity contrast) from Table 4.2. For comparison, we have drawn the predicted geoid slope versus age relationships for plate models with $75 \mathrm{~km}$ (Short dash) and $125 \mathrm{~km}$ ( lonc dash) thick plates.

Figure 4.11: The geoid profiles for runs: (a) 2 (a); (b) 2 (b); and (c) 2(c), from Table 4.2 as in Figure 4.4. The geoid is in meters and the distance in kilometers.

Figure 4.12: The geoid slope versus age relationships for runs $2(a), 2(b)$ and 2 (c) (with a Rayleigh number of $10^{5}$, a top layer thickness of $125 \mathrm{~km}$ and a visosity contrast of $1.0,0.1$ and 0.01 , respectively) from Table 4.2. For comparison, we have drawn the predicted geoid slope versus age relationships for plate models with $75 \mathrm{~km}$ (Short dash) and $125 \mathrm{~km}$ (long dash) thick plates. 
Figure 4.13: The geoid profiles for run 3 from Table 4.2 as in Figure 4.5. The geoid is in meters and the distance in kilometers.

Eigure 4.14: The geoid slope versus age relationships for runs 3 and 2 (c) (with a viscosity contrast of 0.01 , a top layer thickness of $125 \mathrm{~km}$ and a Rayleigh number of $10^{4}$ and $10^{5}$, respectively) from Table 4.2. For comparison, we have drawn the predicted geoid slope versus age relationships for plate models with $75 \mathrm{~km}$ (short dash) and $125 \mathrm{~km}$ ( long dash) thick plates.

Figure 4.15: The geoid profiles for runs: (a) 4 (a); and (b) 4 (b), from Table 4.2 as in Figure 4.6. The geoid is in meters and the distance in kilometers.

Figure 4.16: The geoid slope versus age relationships for runs $4(\mathrm{a}), 4(\mathrm{~b})$ and $2(\mathrm{~b})$ (with a Rayleigh number of $10^{5}$, a viscosity contrast of 0.1 and a top layer thickness of 50 , 300 and $125 \mathrm{~km}$, respectively) from Table 4.2. For comparison, we have drawn the predicted geoid slope versus age relationships for plate models with $75 \mathrm{~km}$ (short dash) and $125 \mathrm{~km}$ (long dash) thick plates.

Figure 4.17: Sample geoid profiles over the Udintsev fracture zone from the SEASAT mission, with the GEM9 field removed up to and including degrees $l=m=10$ and with the 
regional field removed with a best fit to a second order polynomial: (a) from the western side of the fracture zone; (b) from the eastern side of the fracture zone.

Figure 4.18: Observed geoid slopes versus age from the Udintsev fracture zone: (a) from the western side of the fracture zone; (b) from the eastern side. The predicted geoid slope-age relationships for plate models with $75 \mathrm{~km}$ and $125 \mathrm{~km}$ thermal plates are drawn for comparison.

Figure 4.19: Geoid profiles for run 5 with a Rayleigh number of $3.2 \times 10^{5}$, a viscosity contrast of 0.1 and a top layer thickness of $125 \mathrm{~km}$. The geoid is in meters and the distance in kilometers.

Figure 4.20: The geoid slope versus age relationships for run 5, where the viscosity contrast is 0.1 , the Rayleigh number is $3.2 \times 10^{5}$ and the low viscosity layer thickness is $125 \mathrm{~km}$. For comparison, we have drawn the predicted geoid slope versus age relationships for plate models with $75 \mathrm{~km}$ (shortdash) and $125 \mathrm{~km}$ (long dash) thick plates.

Figure 4.21: The topographic step versus age for run $1(a)$ (with a Rayleigh number of $10^{6}$ and no viscosity contrast), run 1 (b) (with a Rayleigh number of $10^{7}$ and no viscosity contrast), run 2 (c) (with a Rayleigh number of $10^{5}$, a top layer thickness of $125 \mathrm{~km}$ and a viscosity contrast of 0.01 ), 
and run 5 (with a Rayleigh number of $3.2 \times 10^{5}$, a top layer thickness of $125 \mathrm{~km}$ and a viscosity contrast of 0.1 ). For comparison, we have drawn the step predicted from the average depth-age relationship for the North Pacific from Parsons and Sclater (1977). 
Fracture Zone Trace

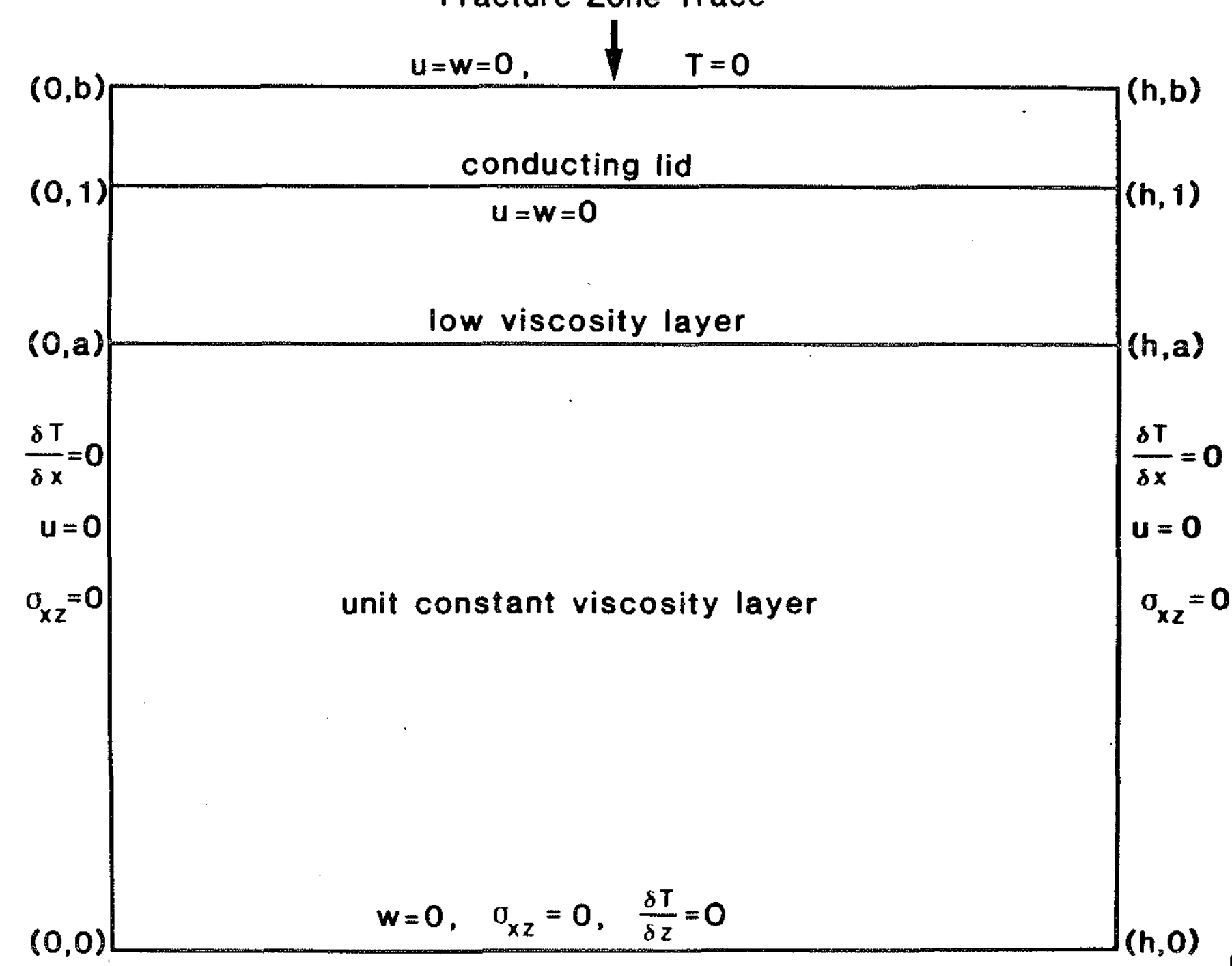



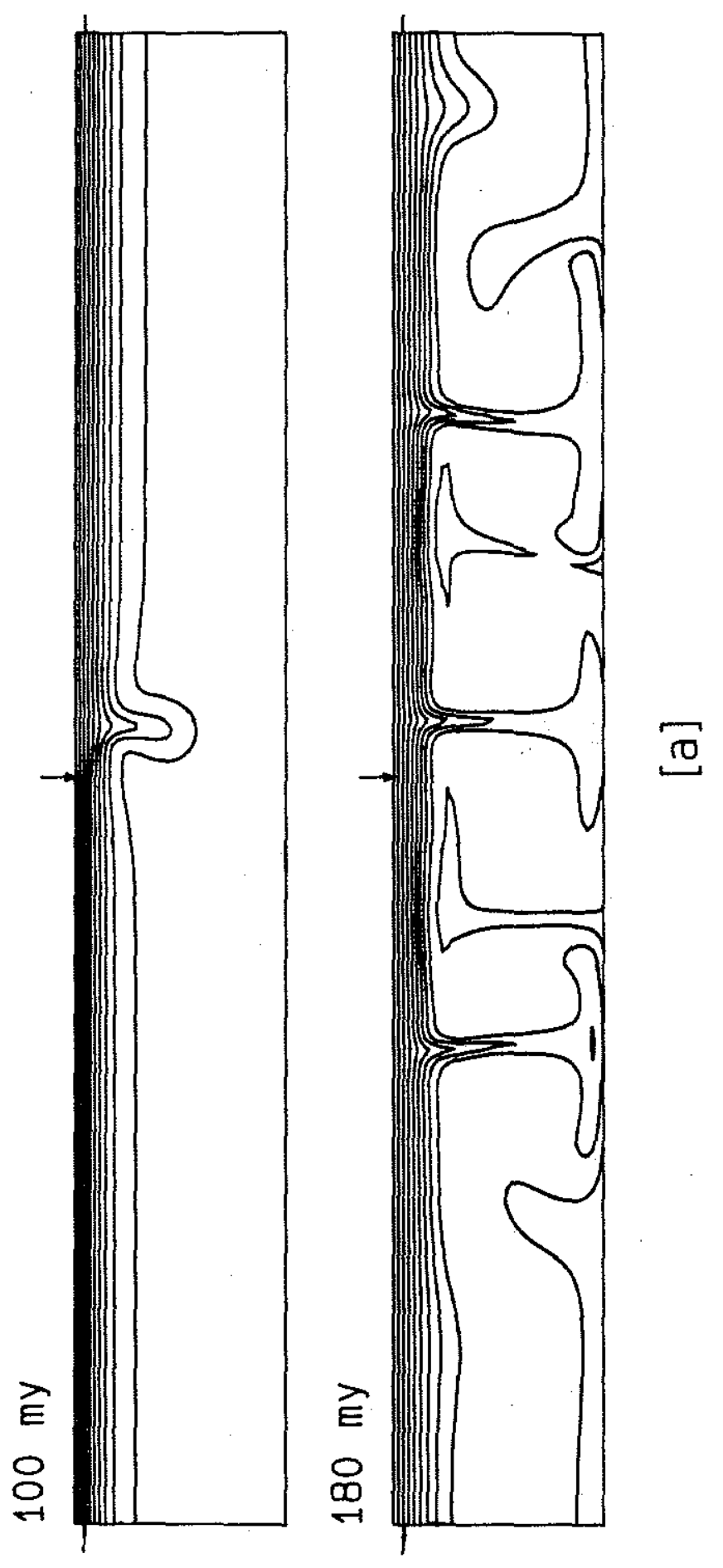

竞 

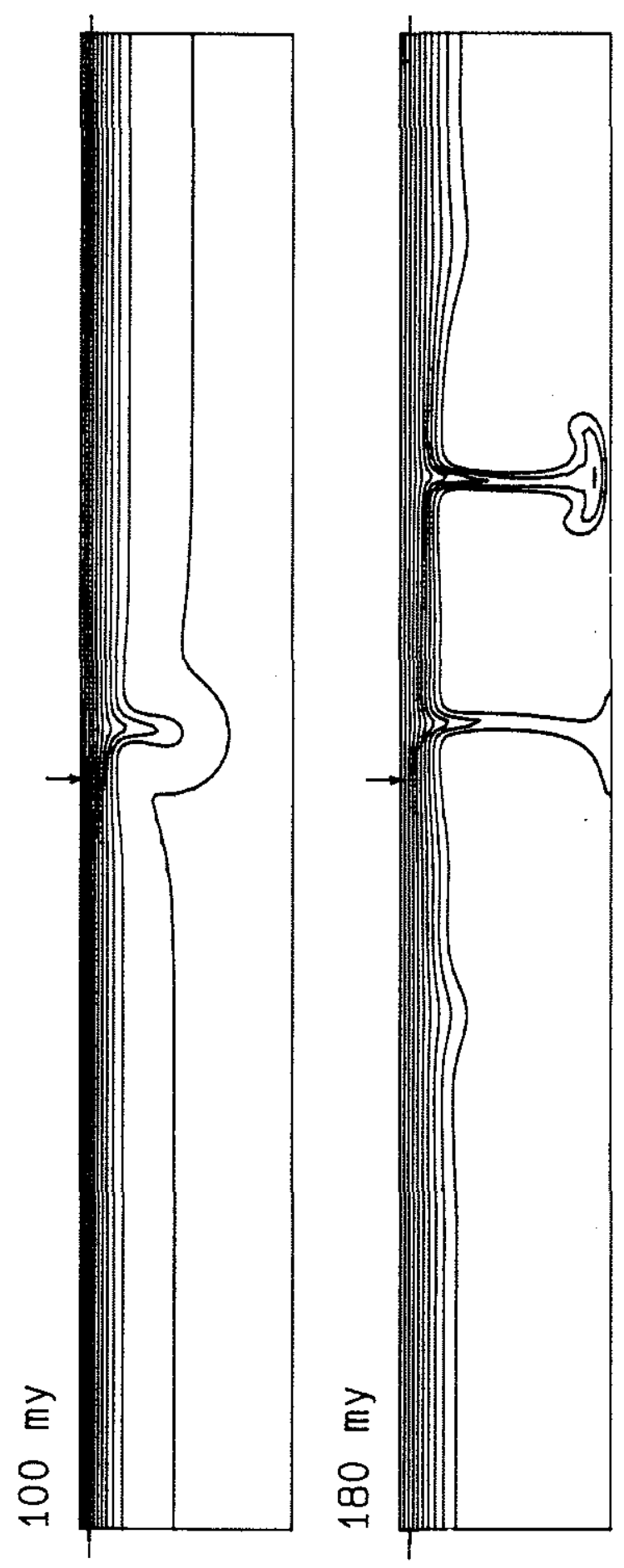

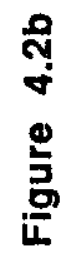

$\Xi$ 

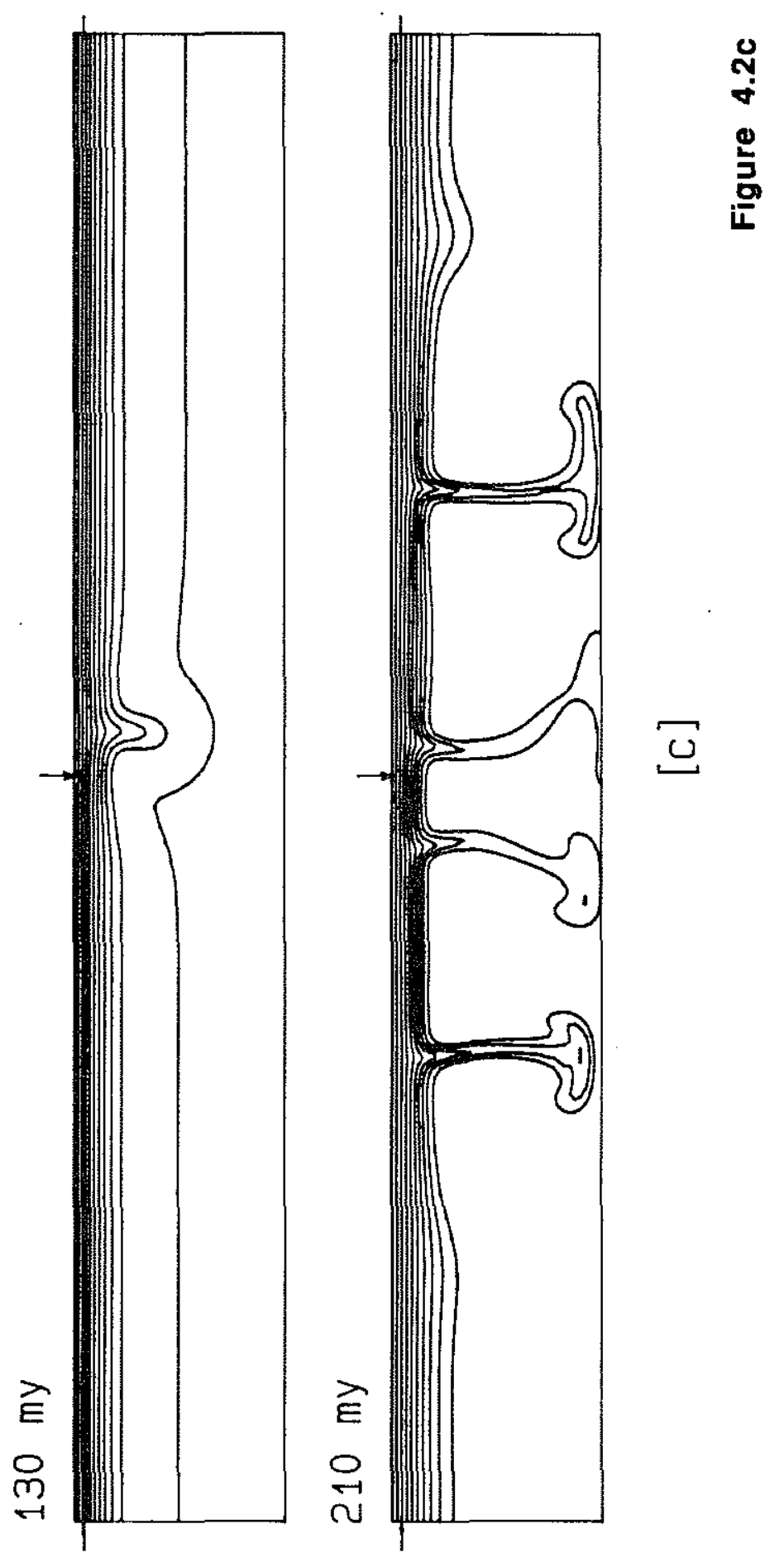


$$
\begin{aligned}
& \| F \\
& \| F
\end{aligned}
$$



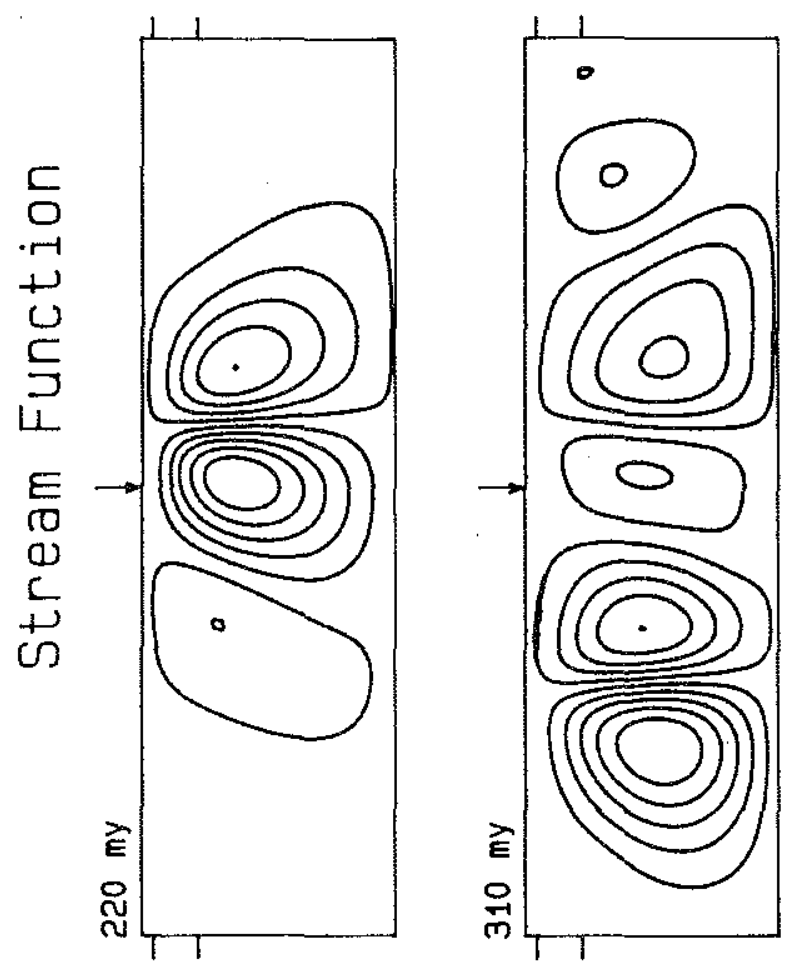

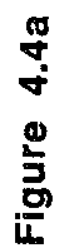
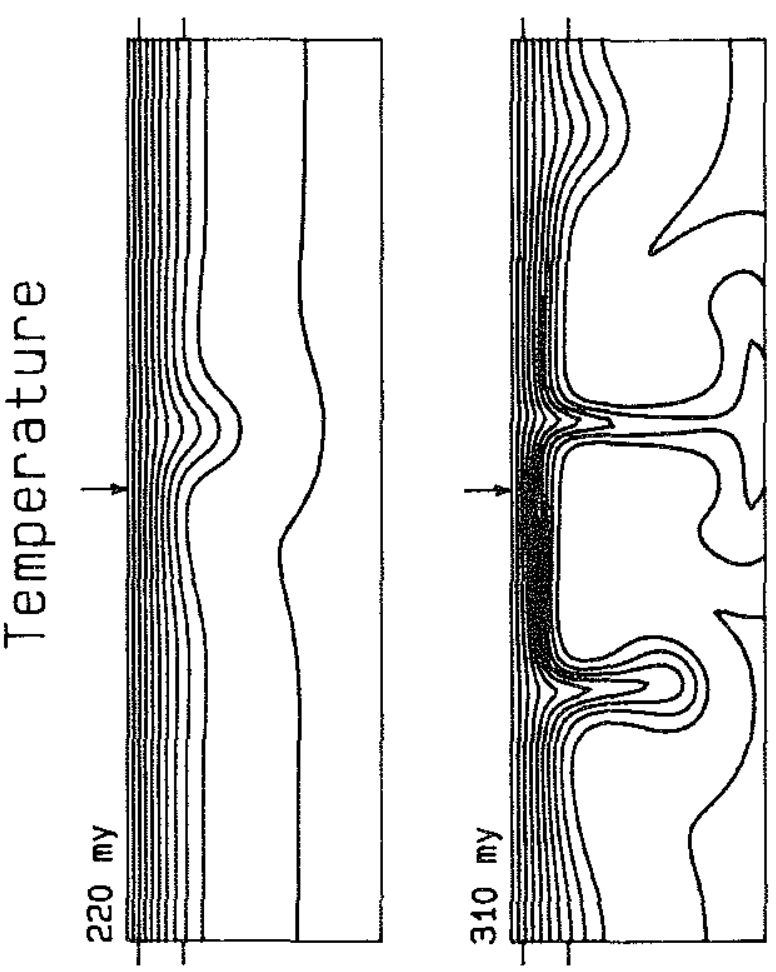

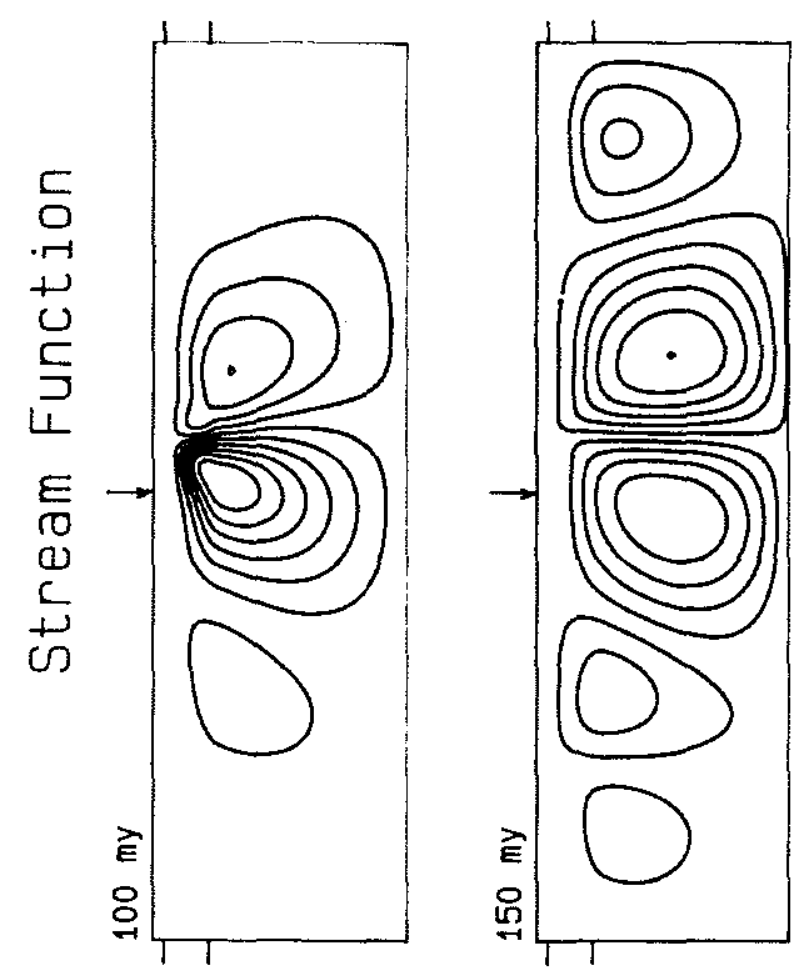

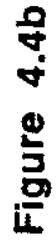
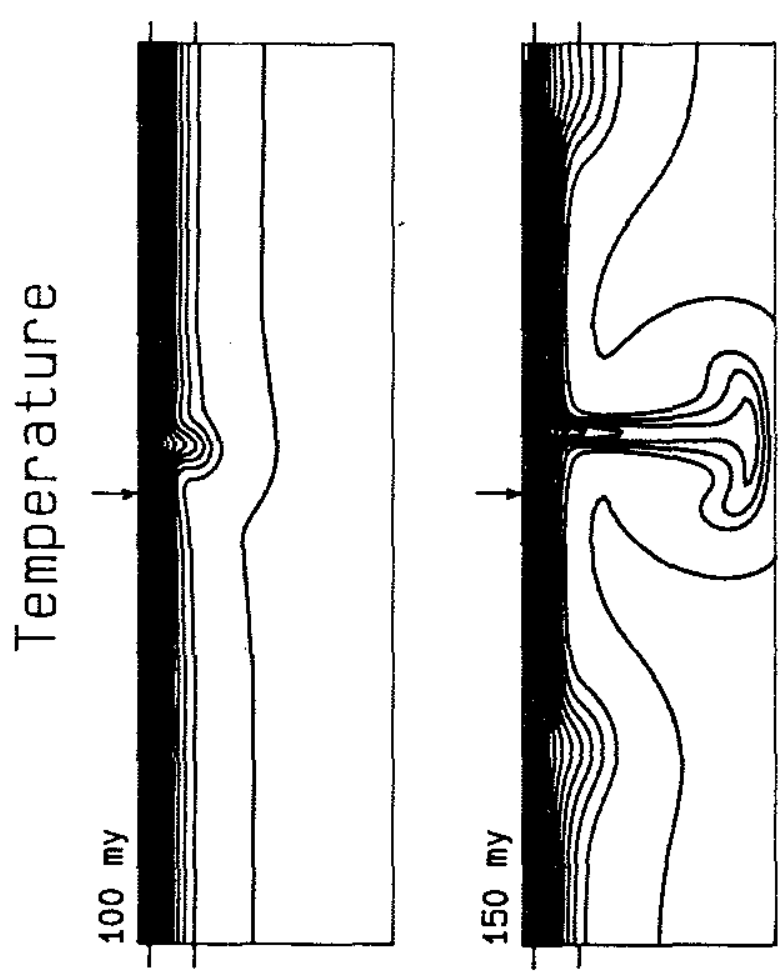

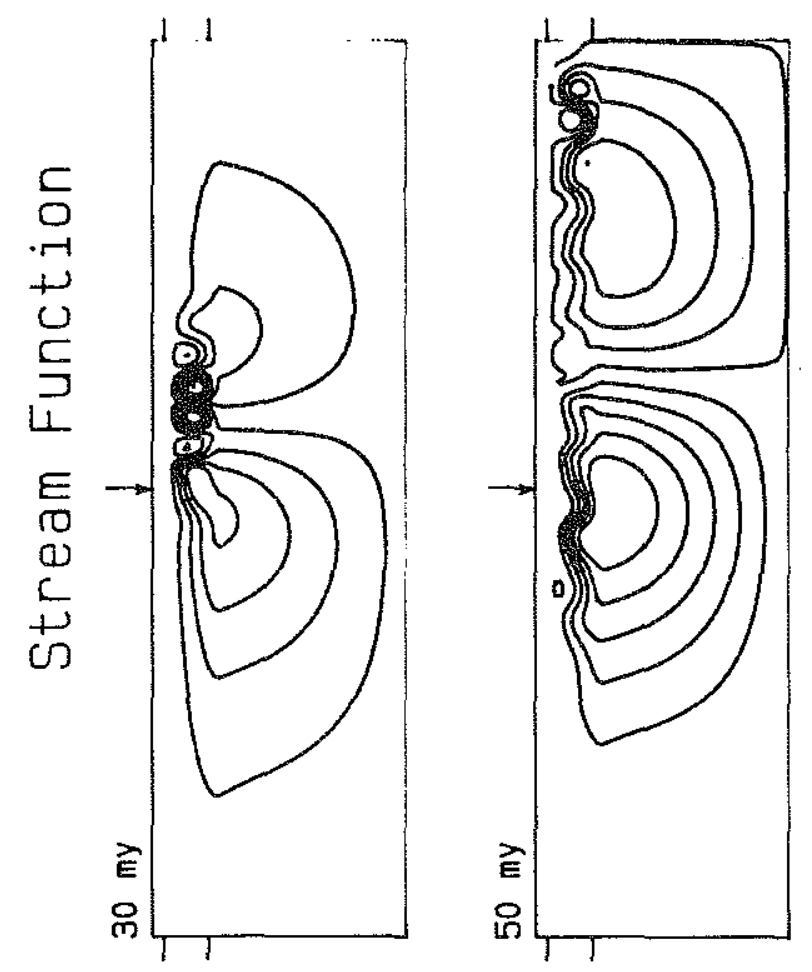

0
0
0
$\frac{1}{3}$
옴
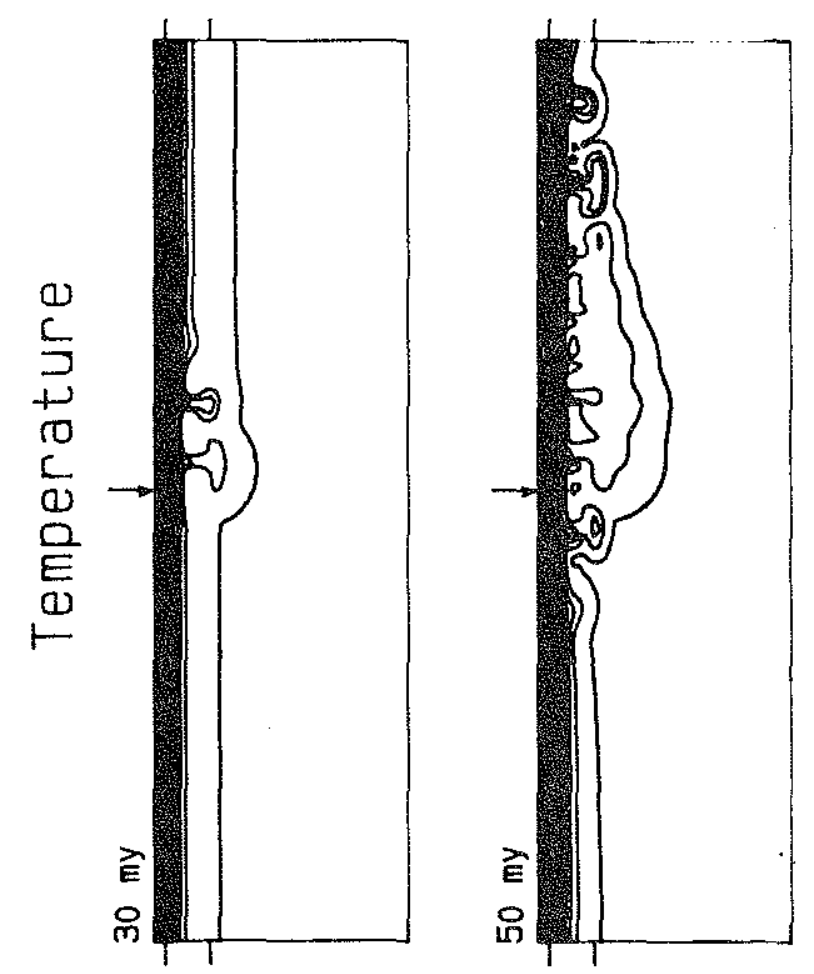

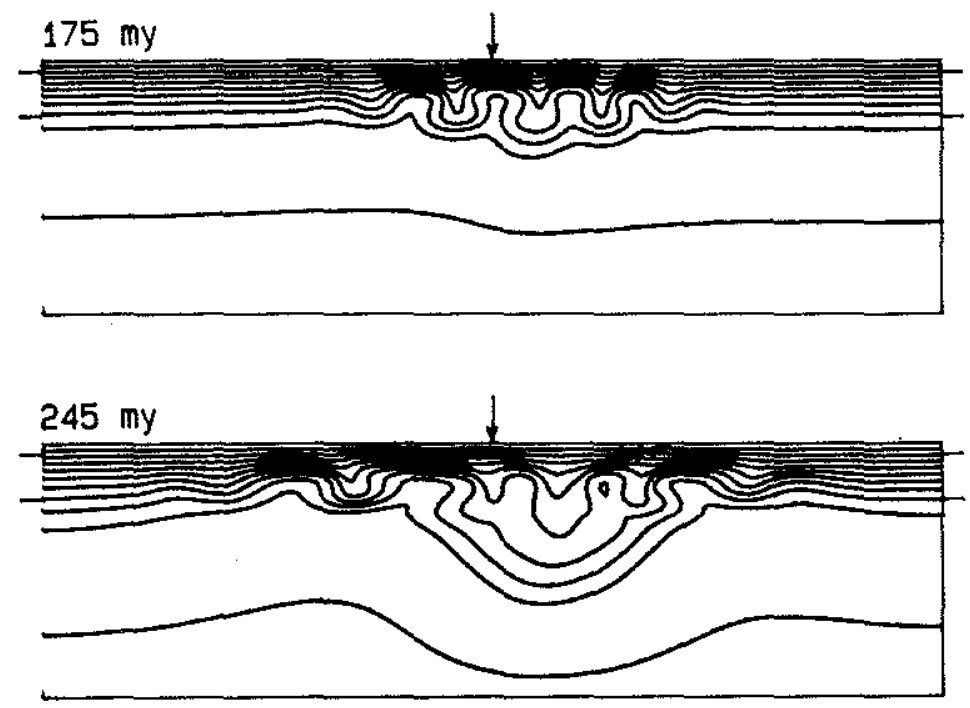

Figure 4.5 


$$
\begin{aligned}
& \mid F \\
& \mid F
\end{aligned}
$$


Topography Kernels
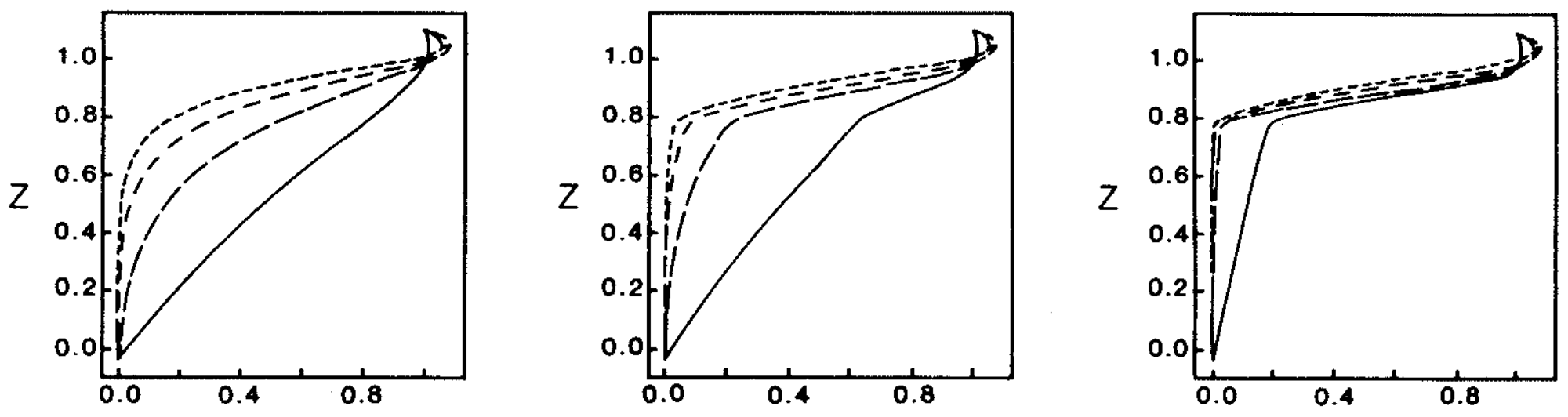

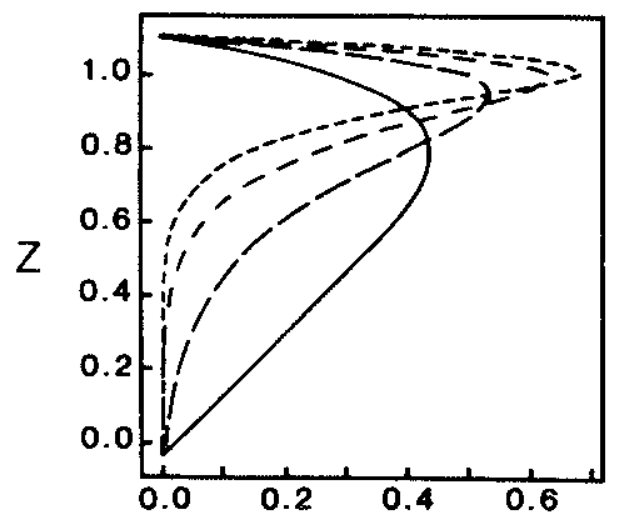

(a)
Gravity Kernels

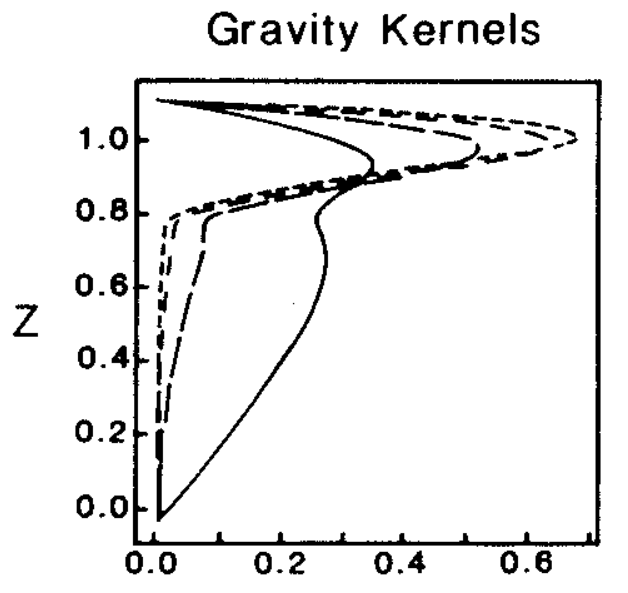

(b)

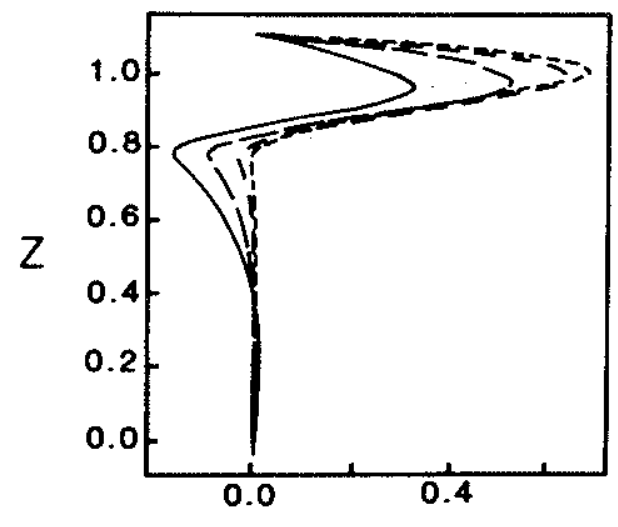

(c)

Figure 4.7 

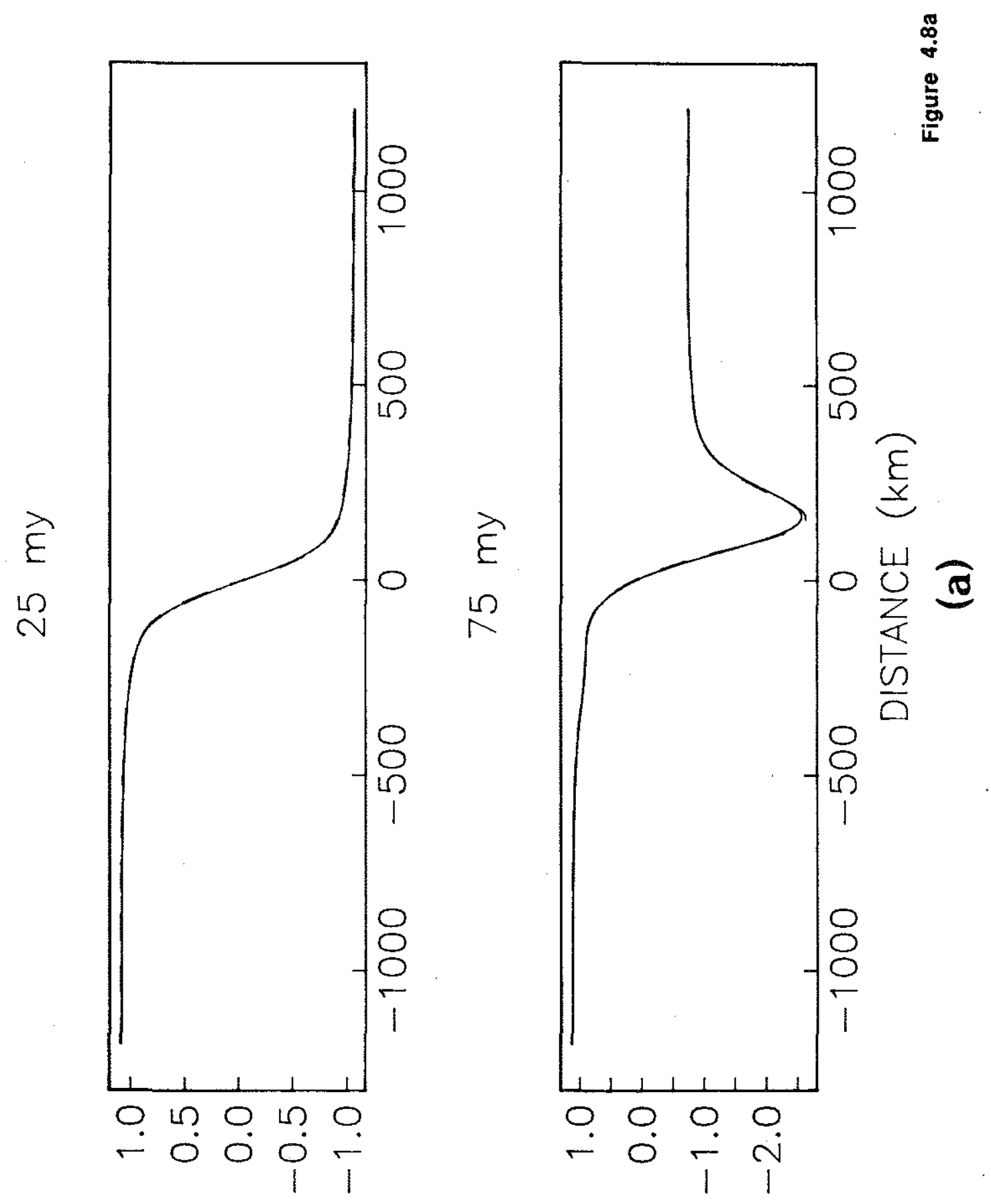

(w) alOت9 


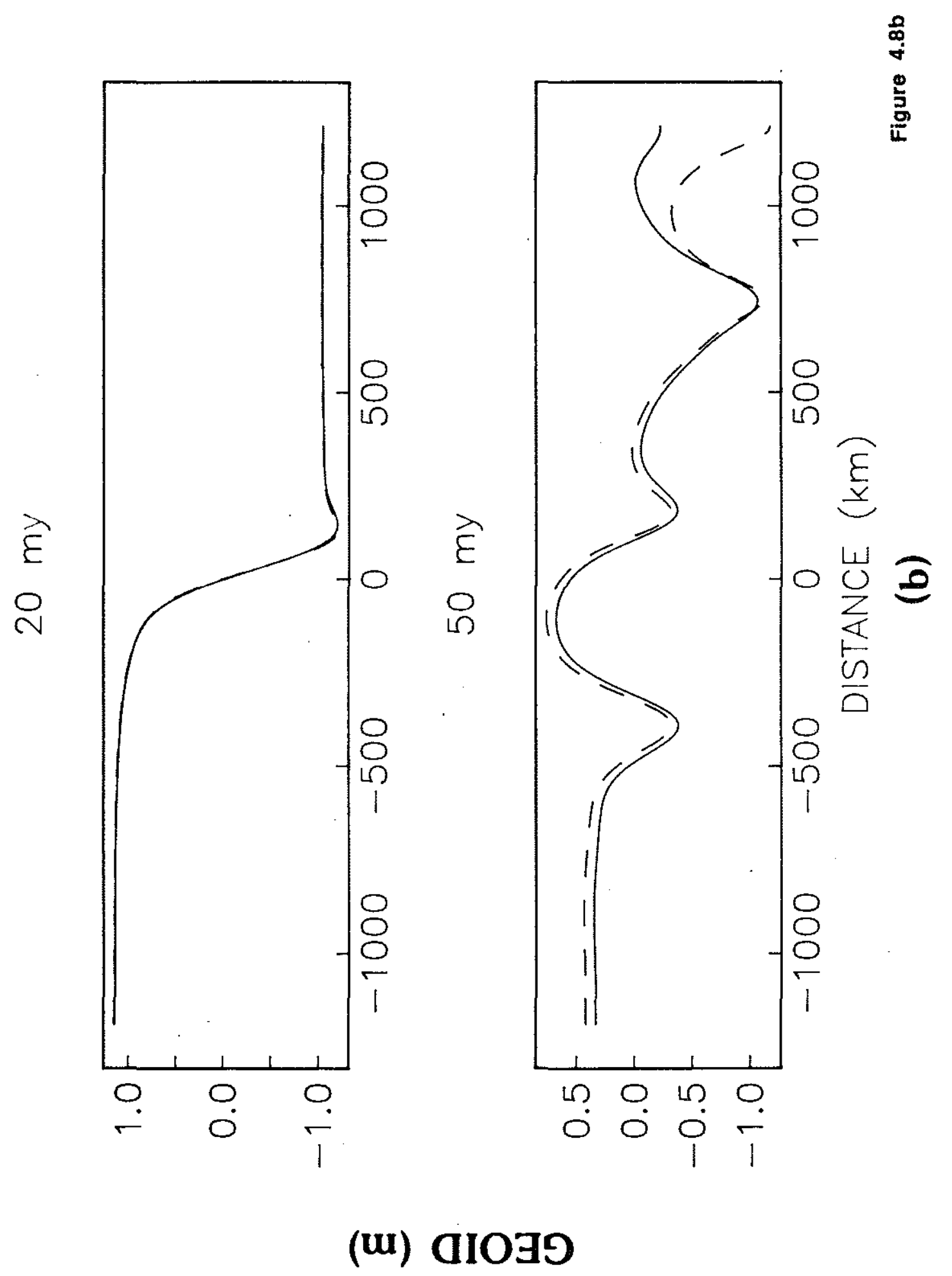




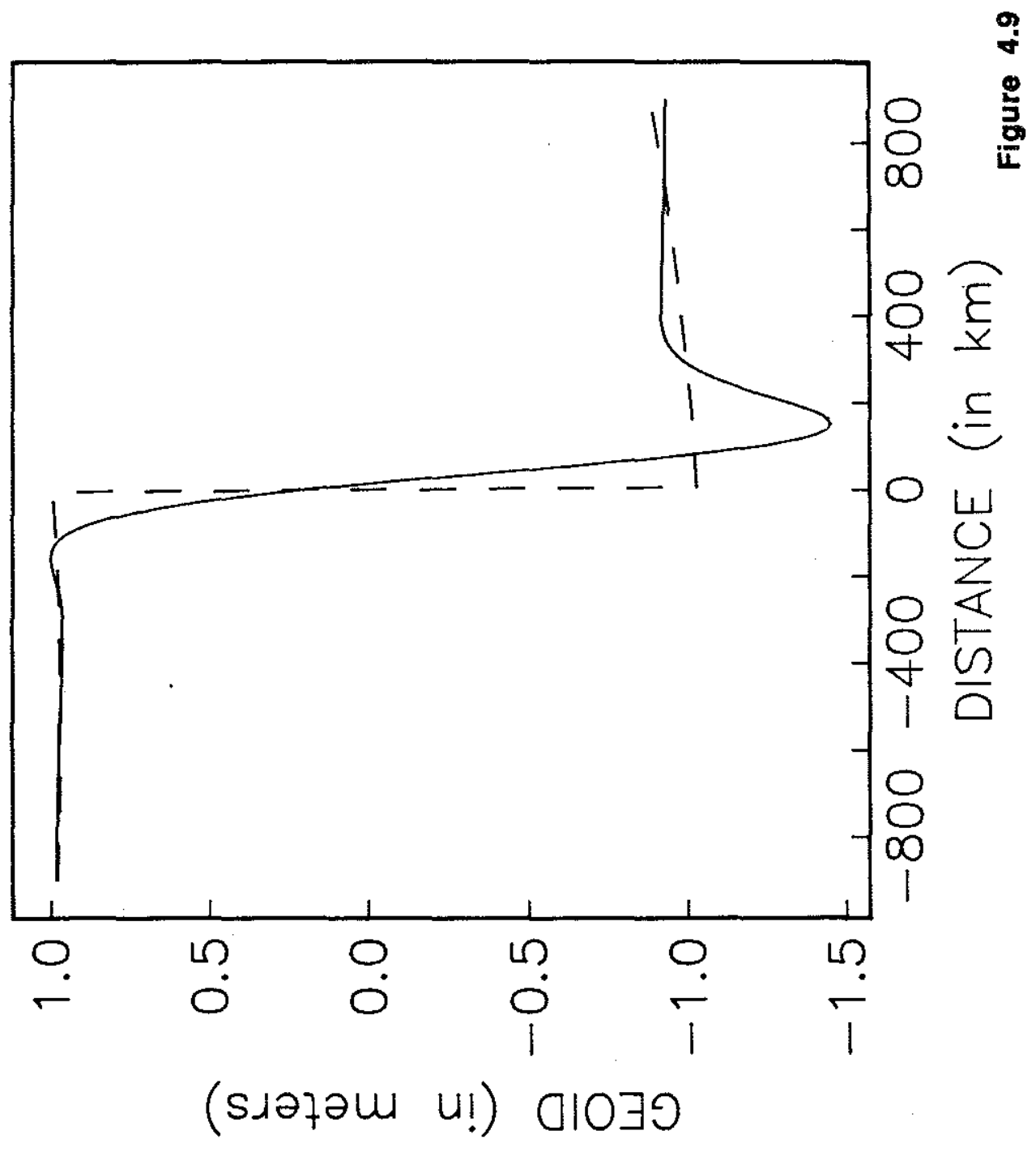


옹

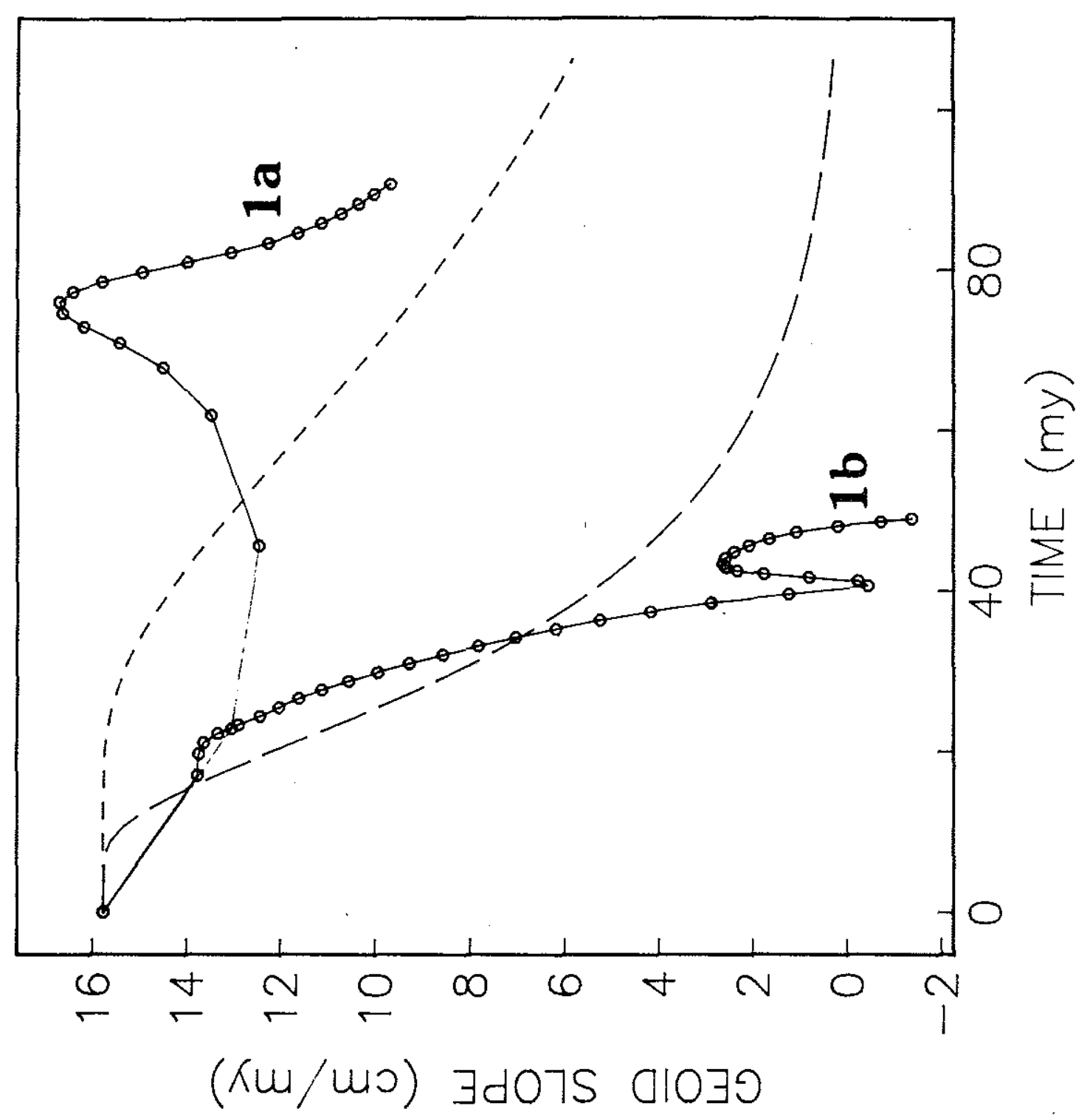


220 my
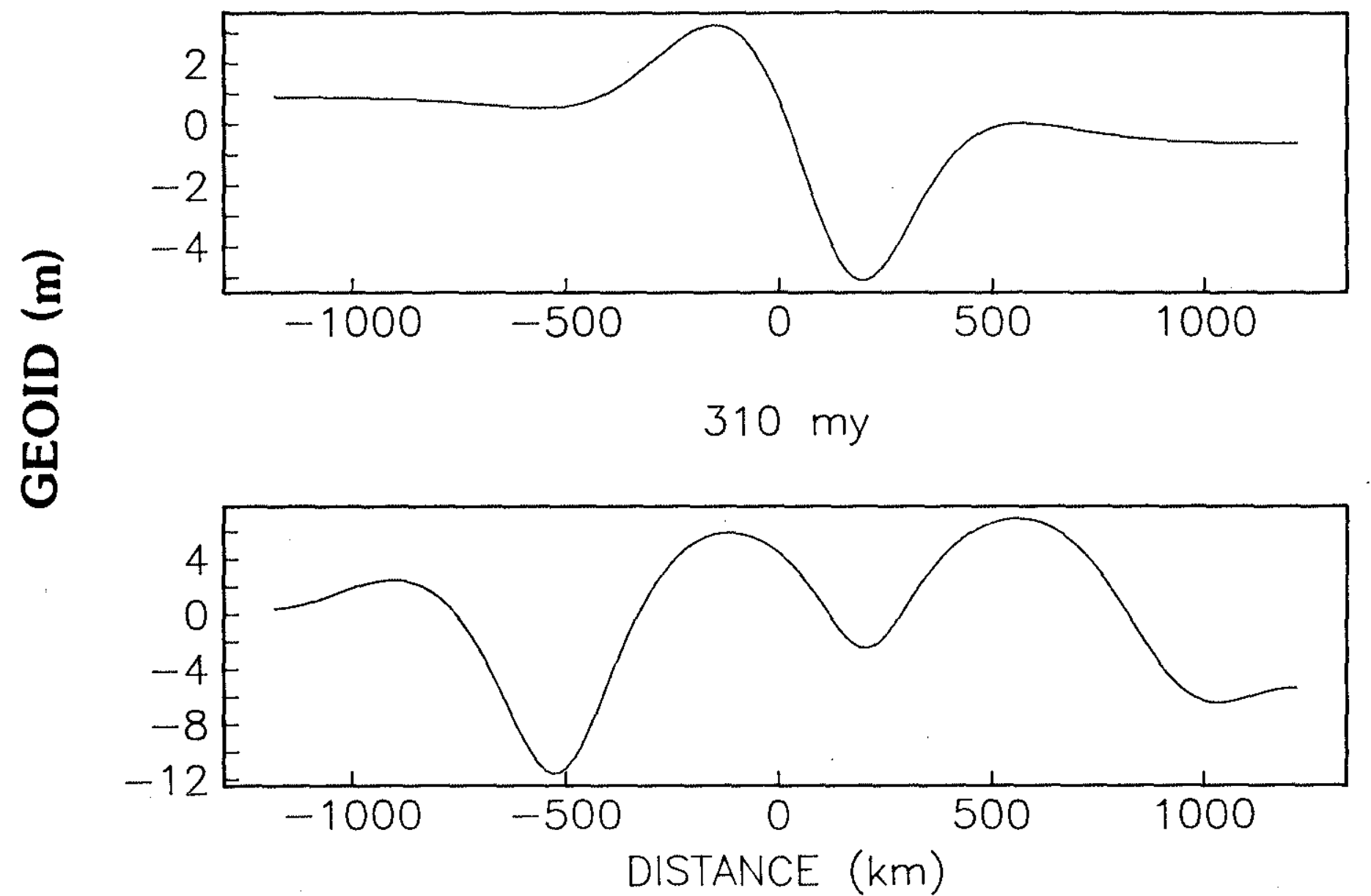

(a)

Figure 4.11a 


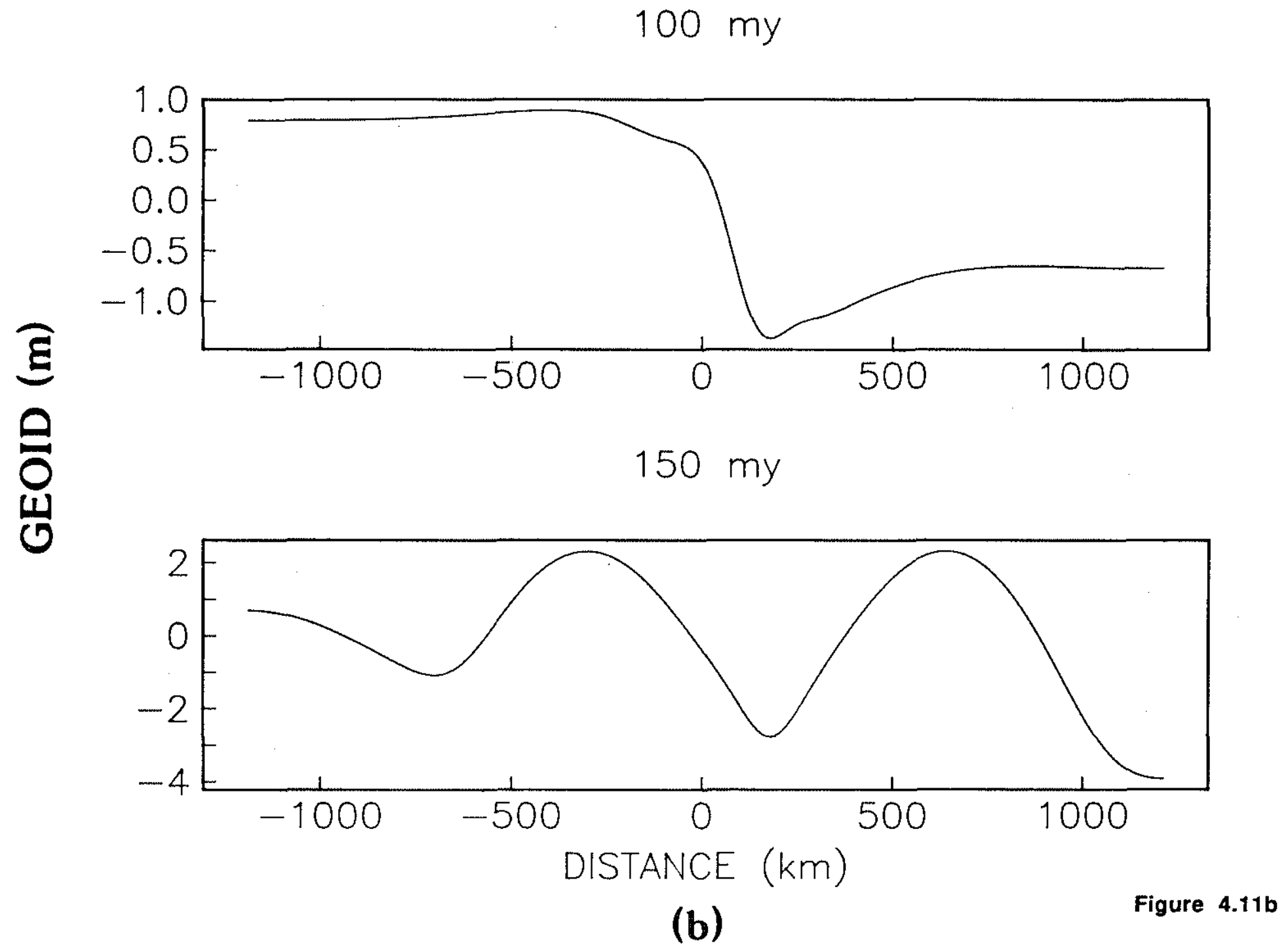


30 my
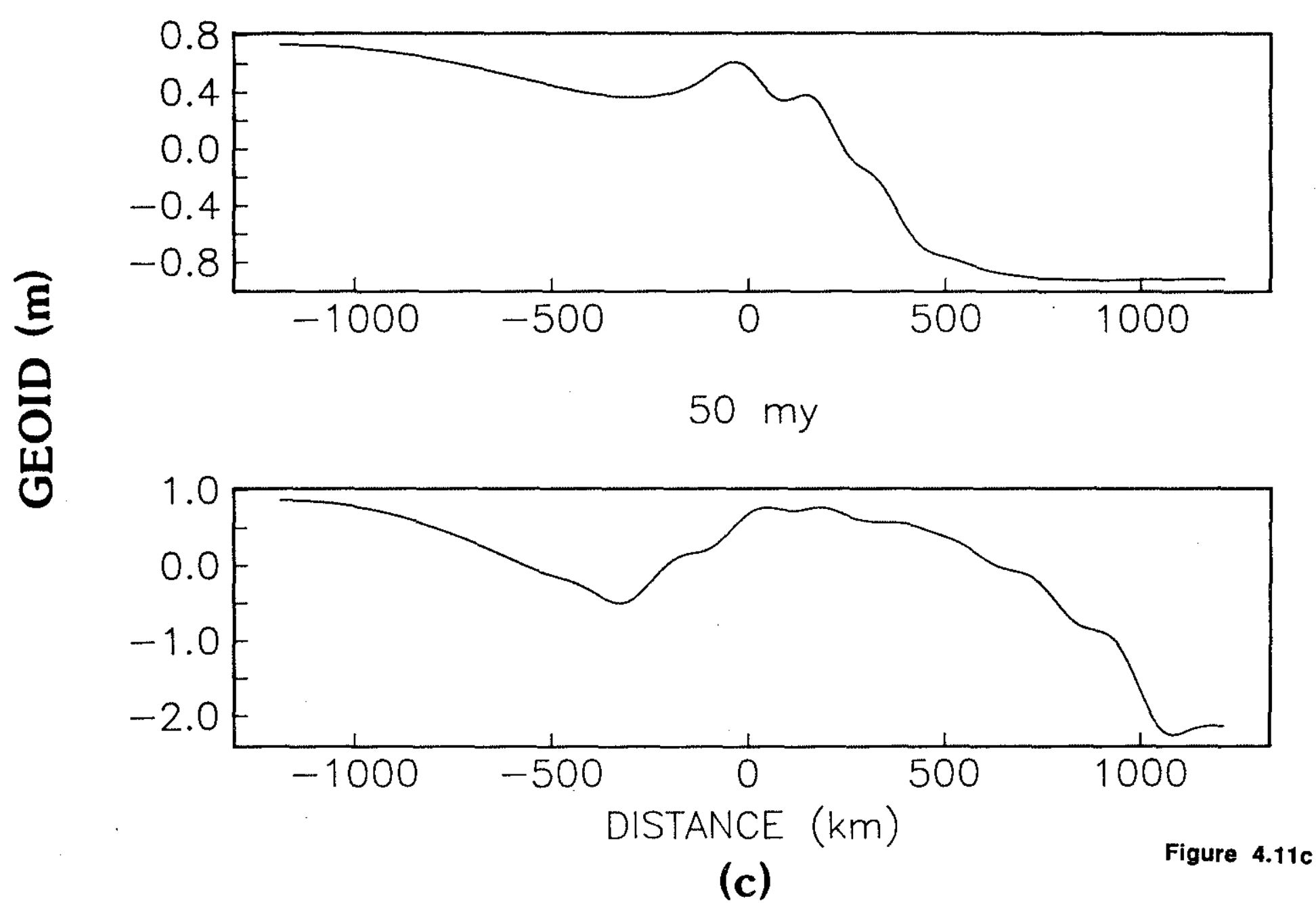


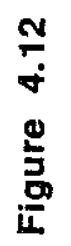

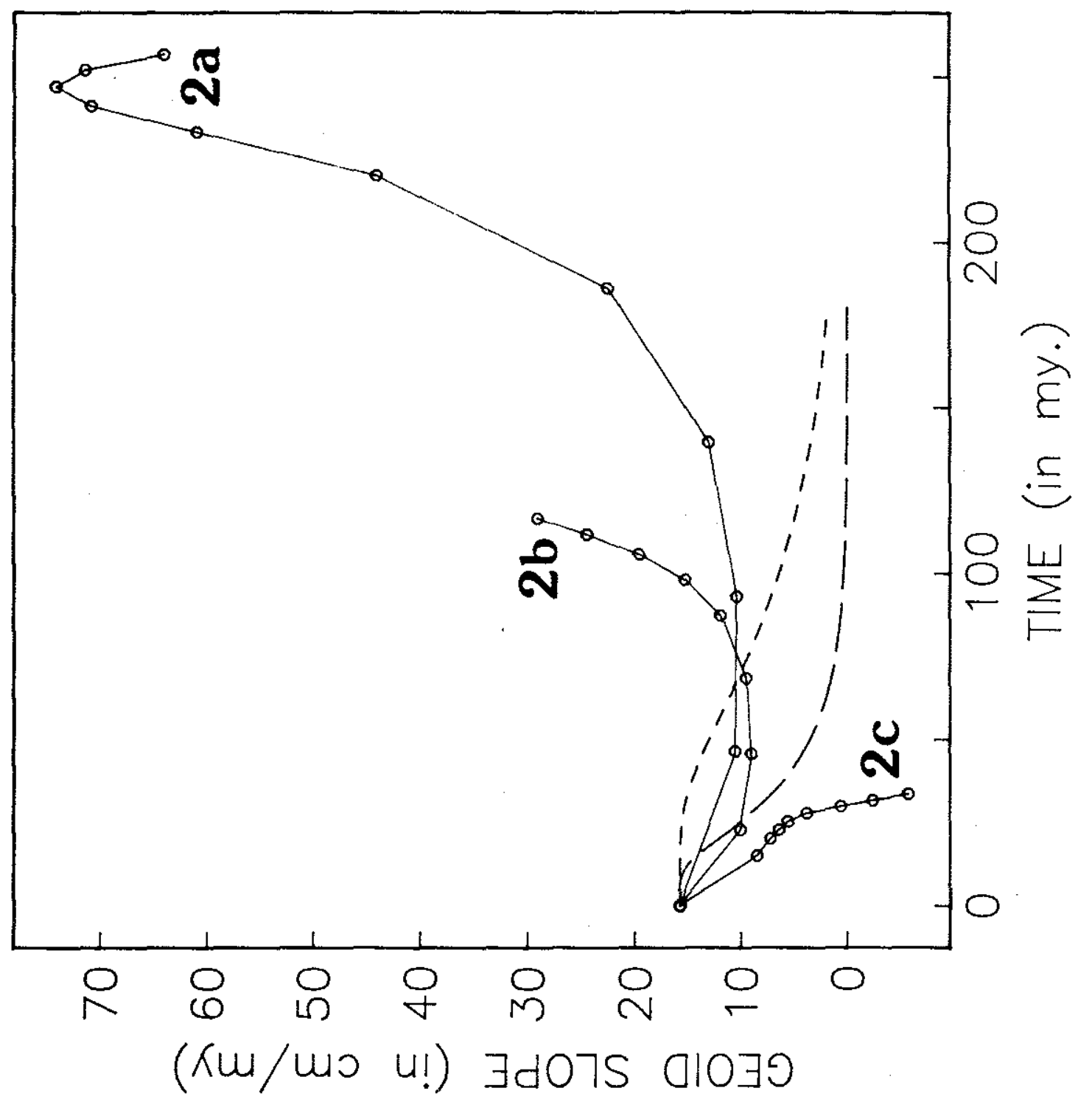




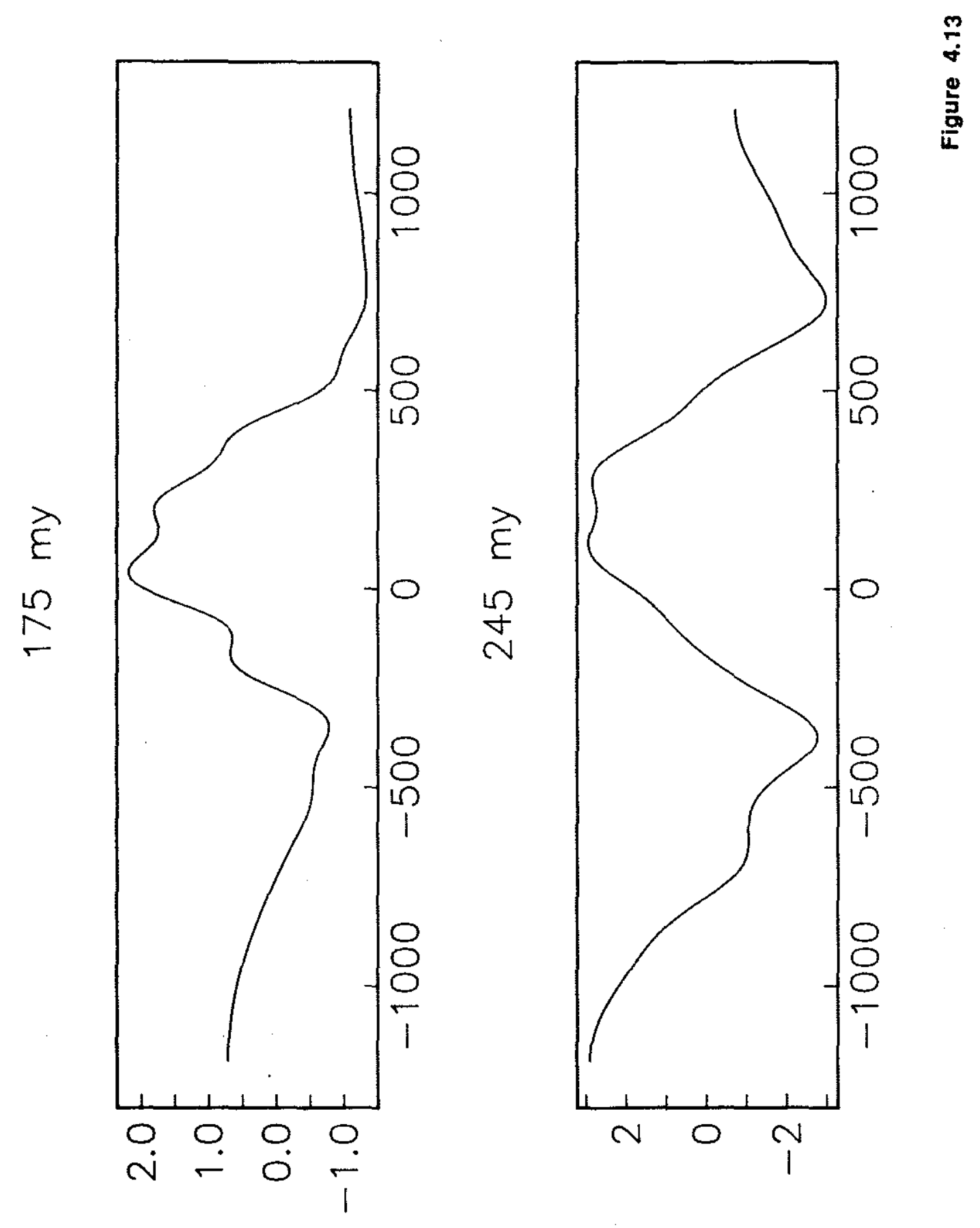

(w) बIOت9 


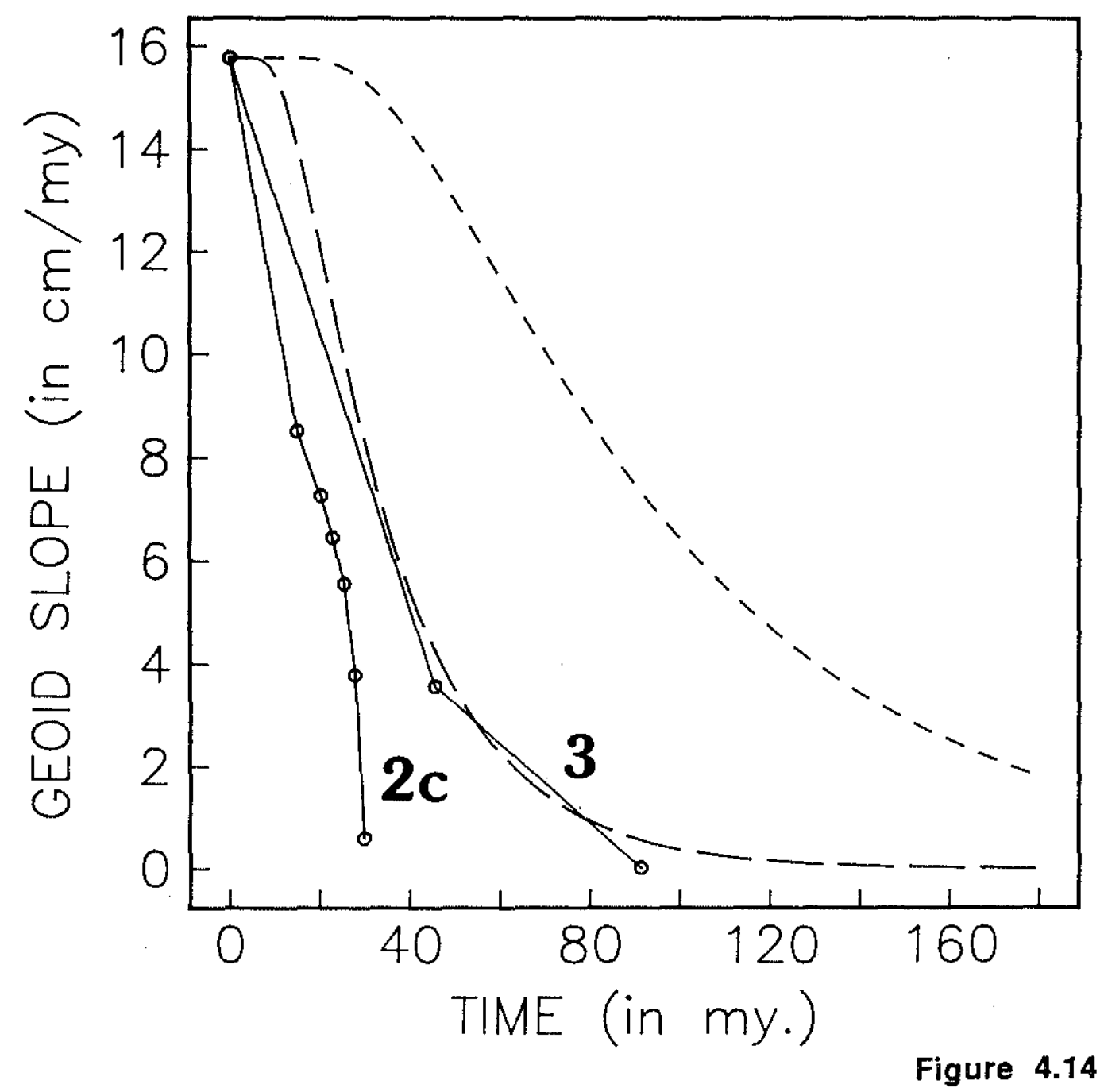



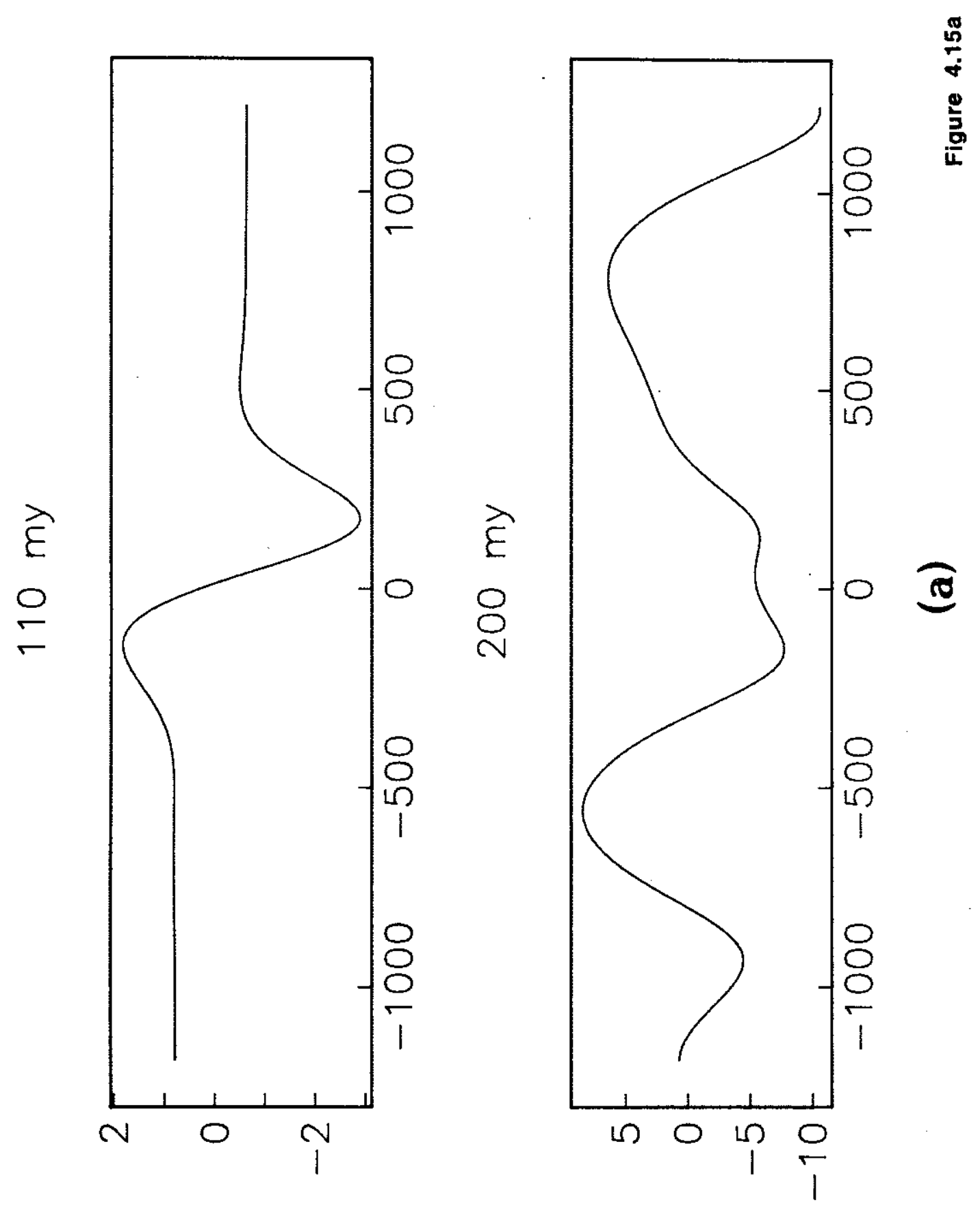

(u) dIOت9 

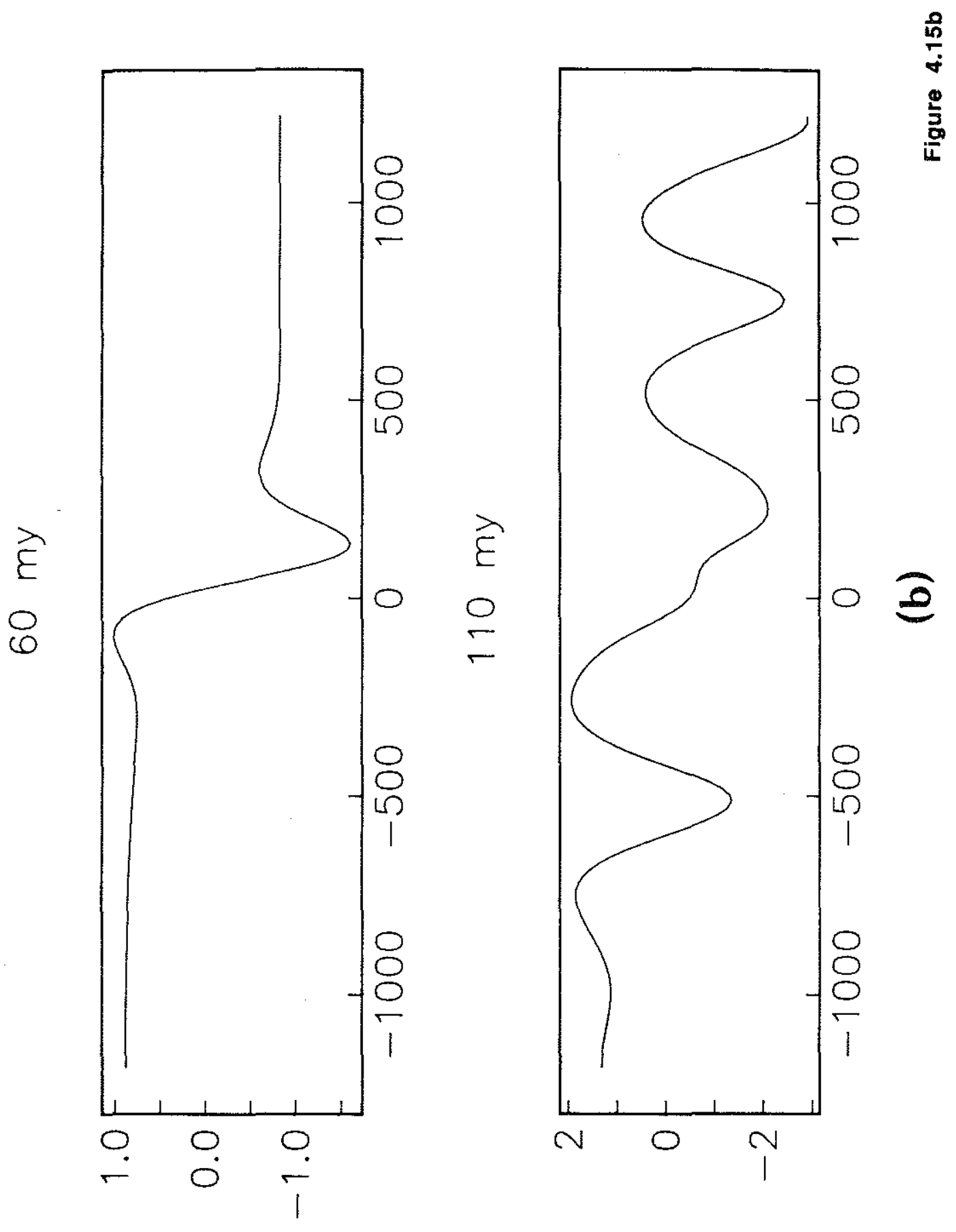

(ui) dIOت9 
0
$\stackrel{+}{0}$
0
$\frac{0}{3}$
문

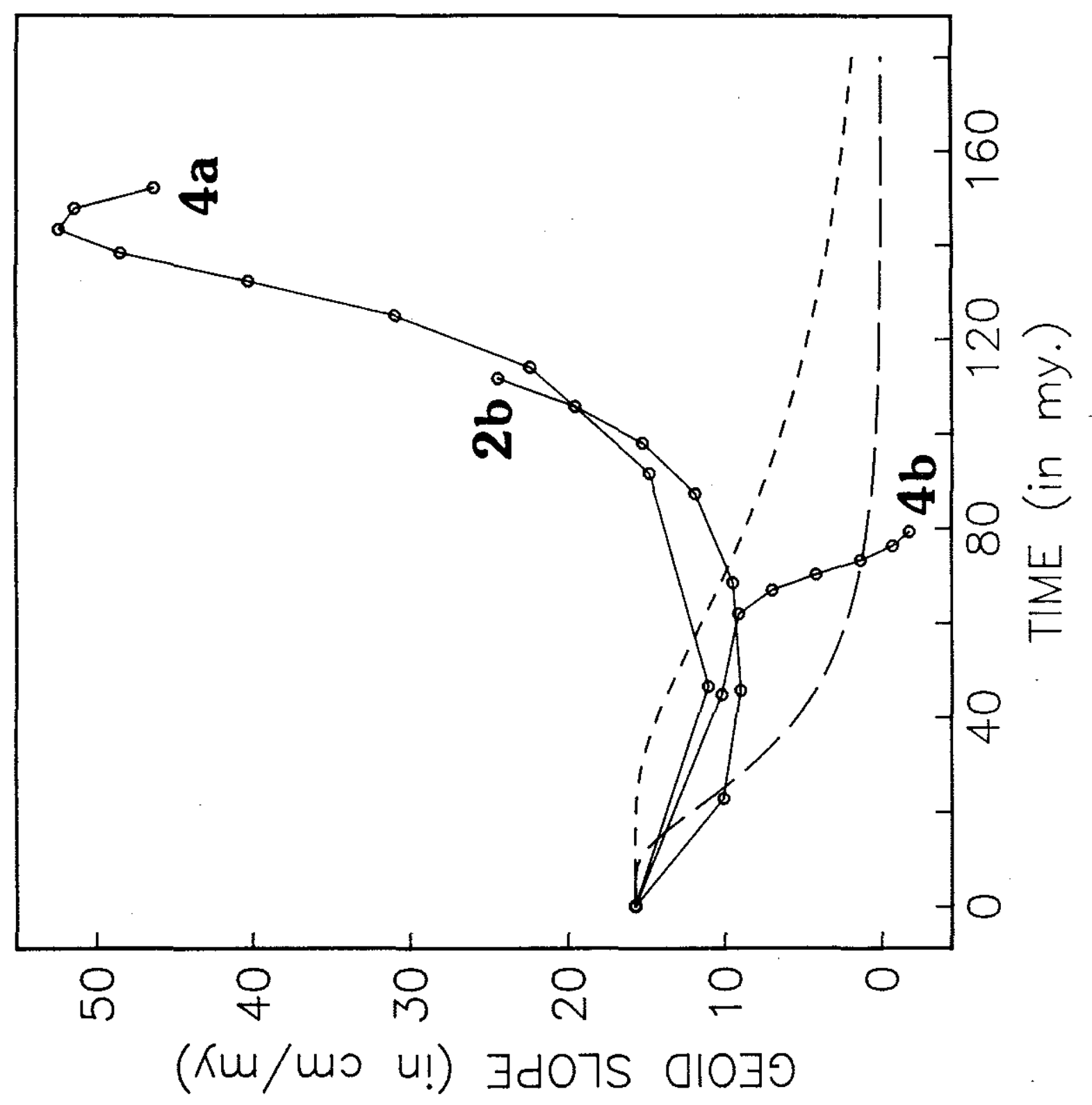



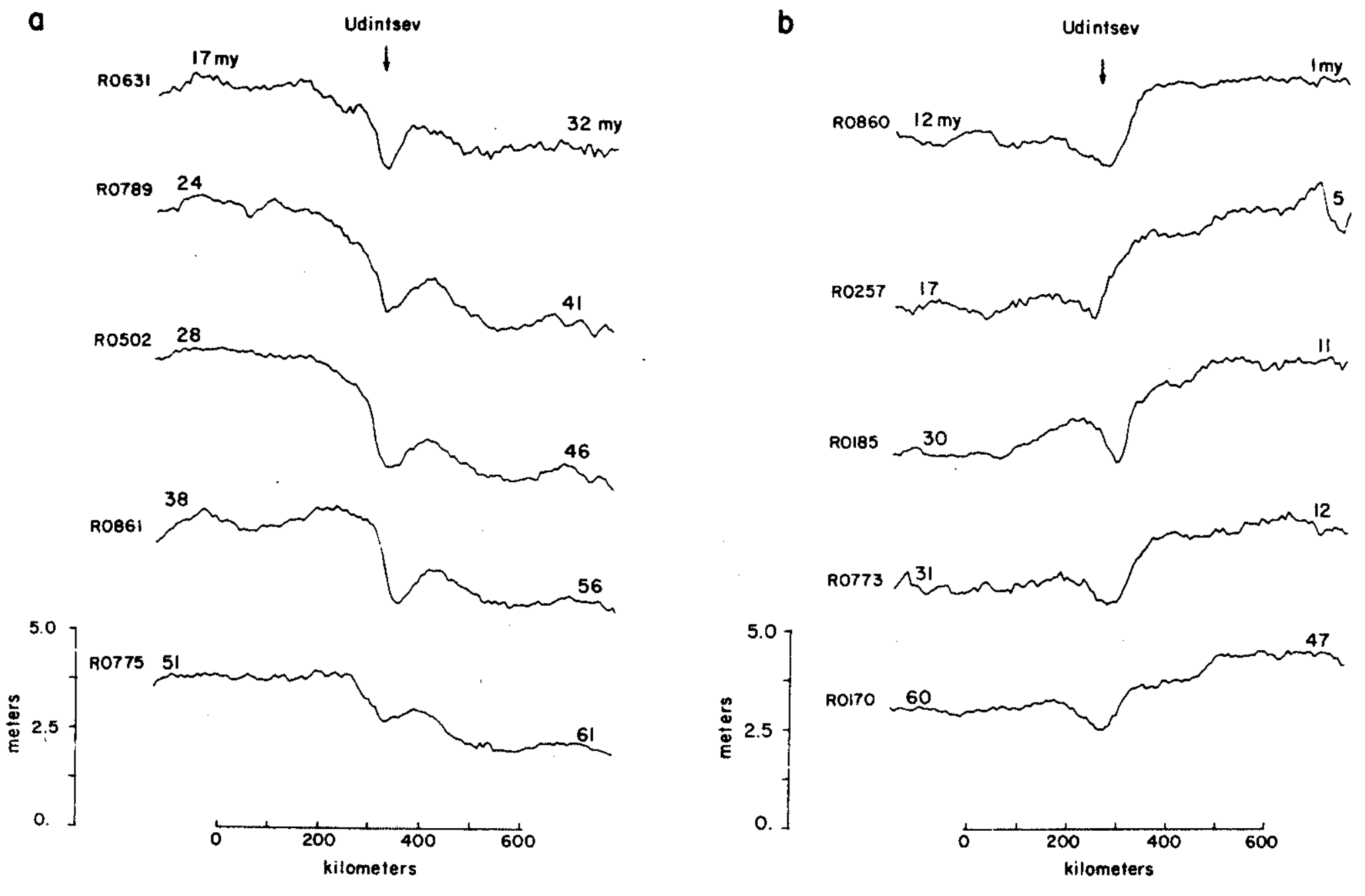

Figure 4.17 


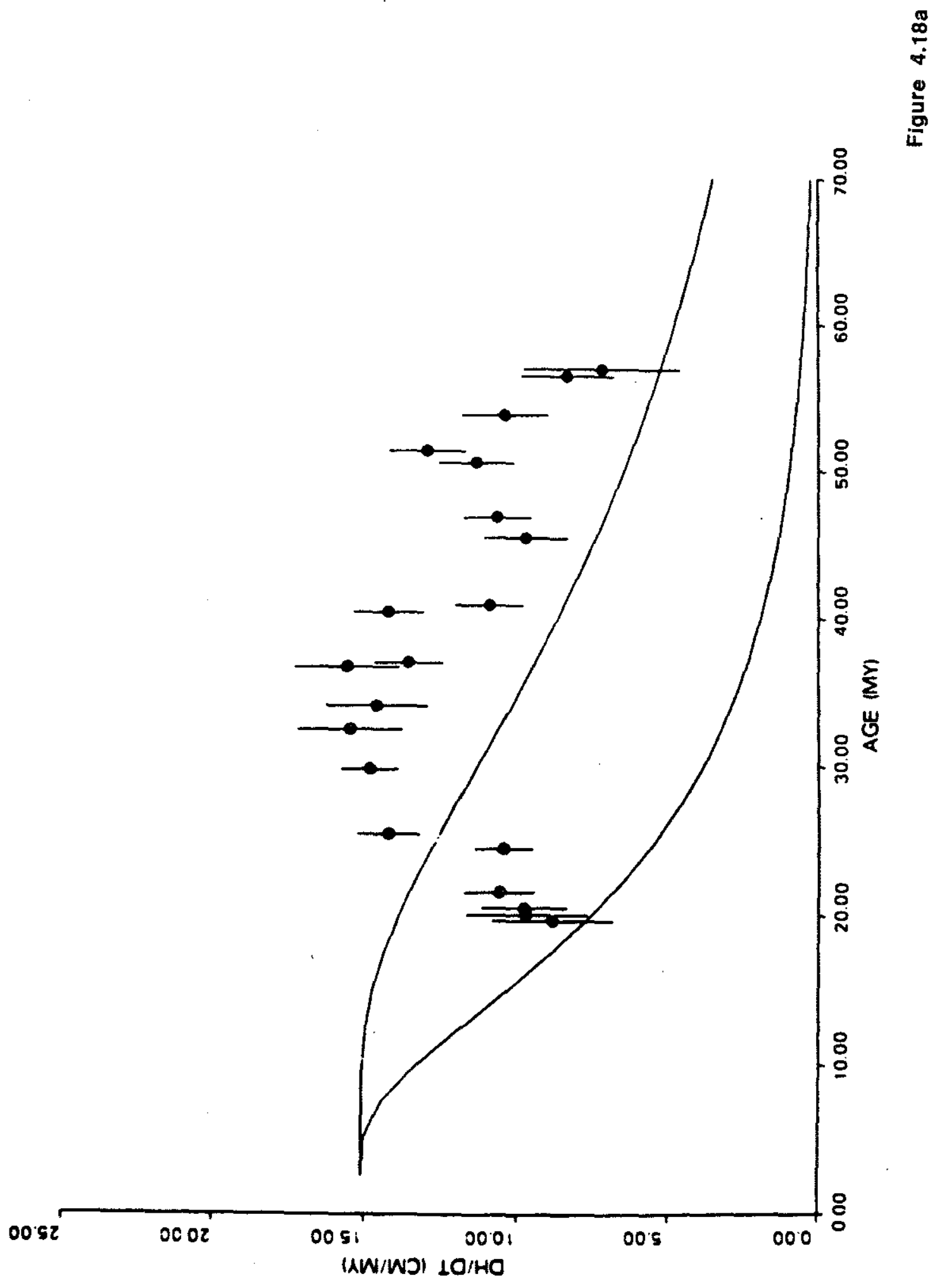


210

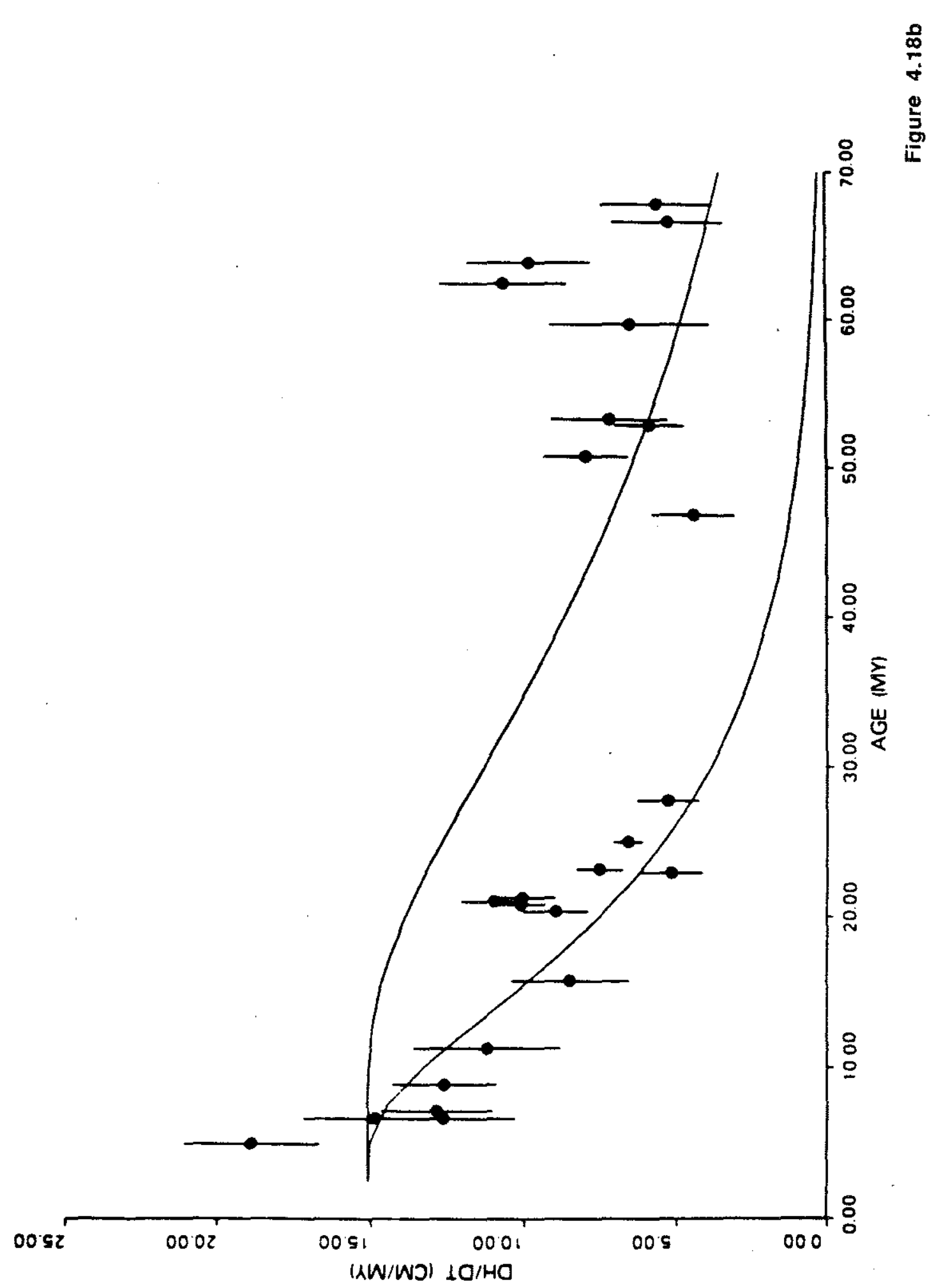


22 m.y.

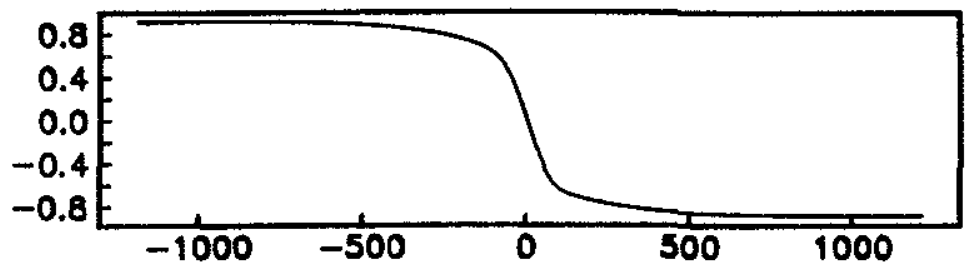

35 m.y.

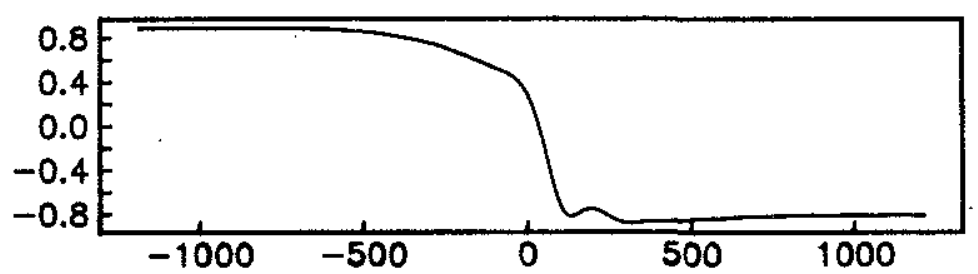

46 m.y.

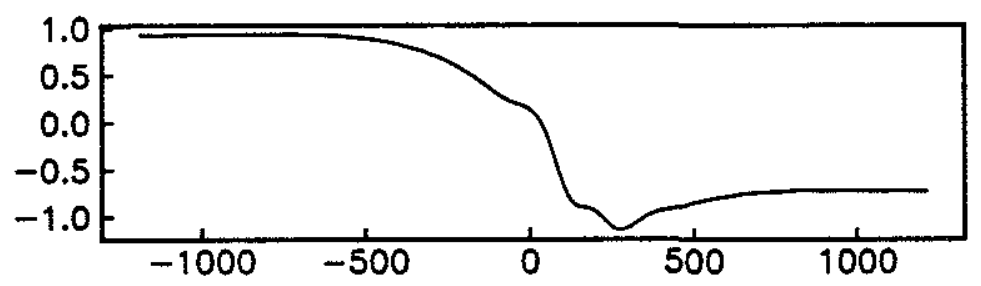

56 m.y.

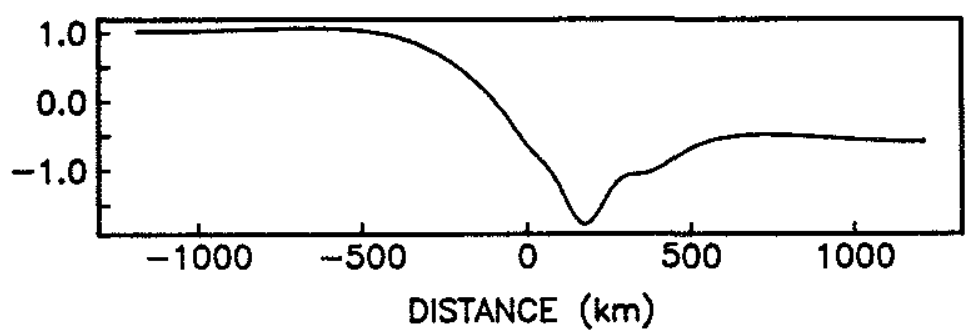

Figure 4.19 


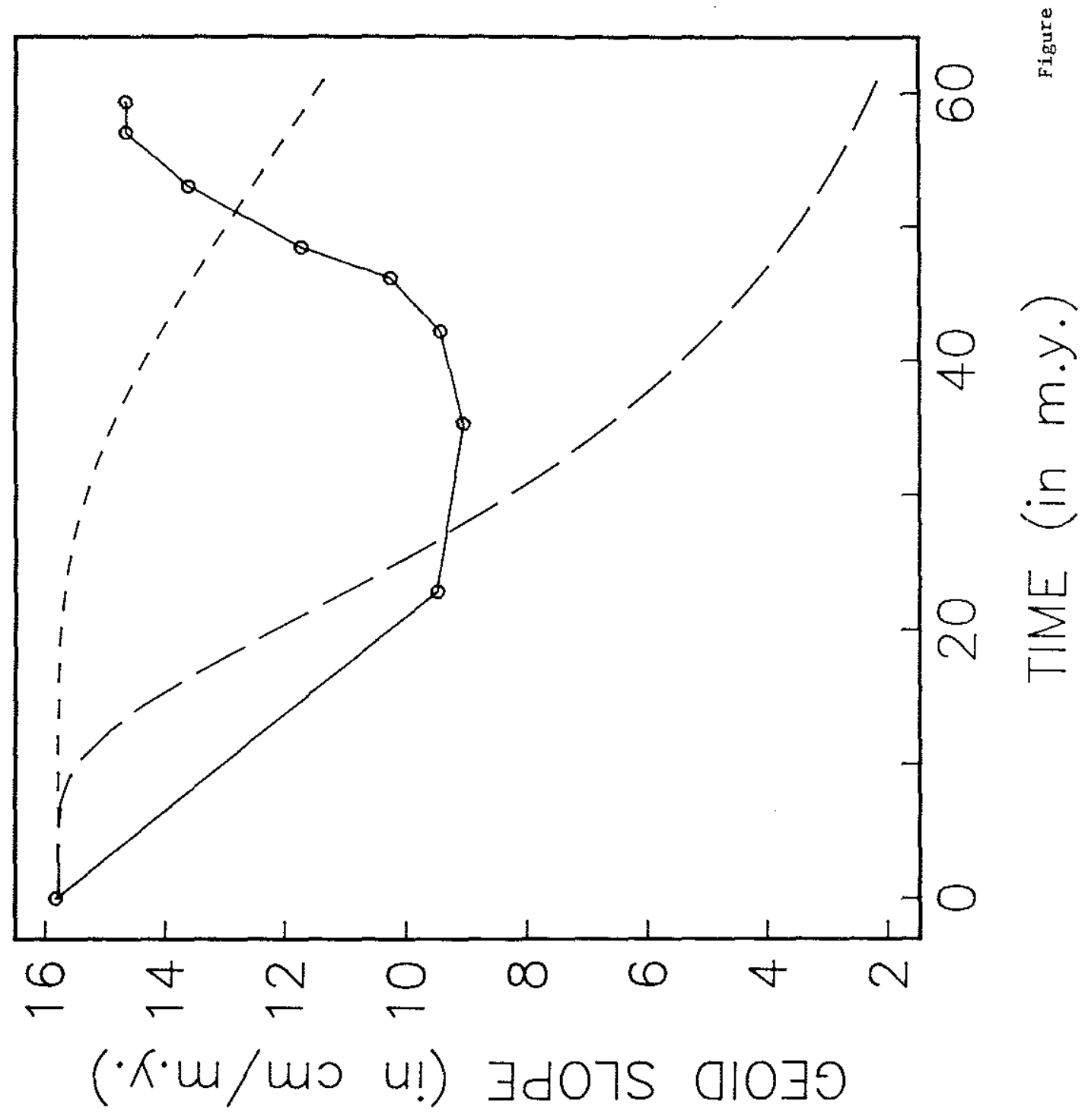




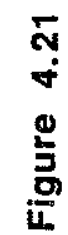

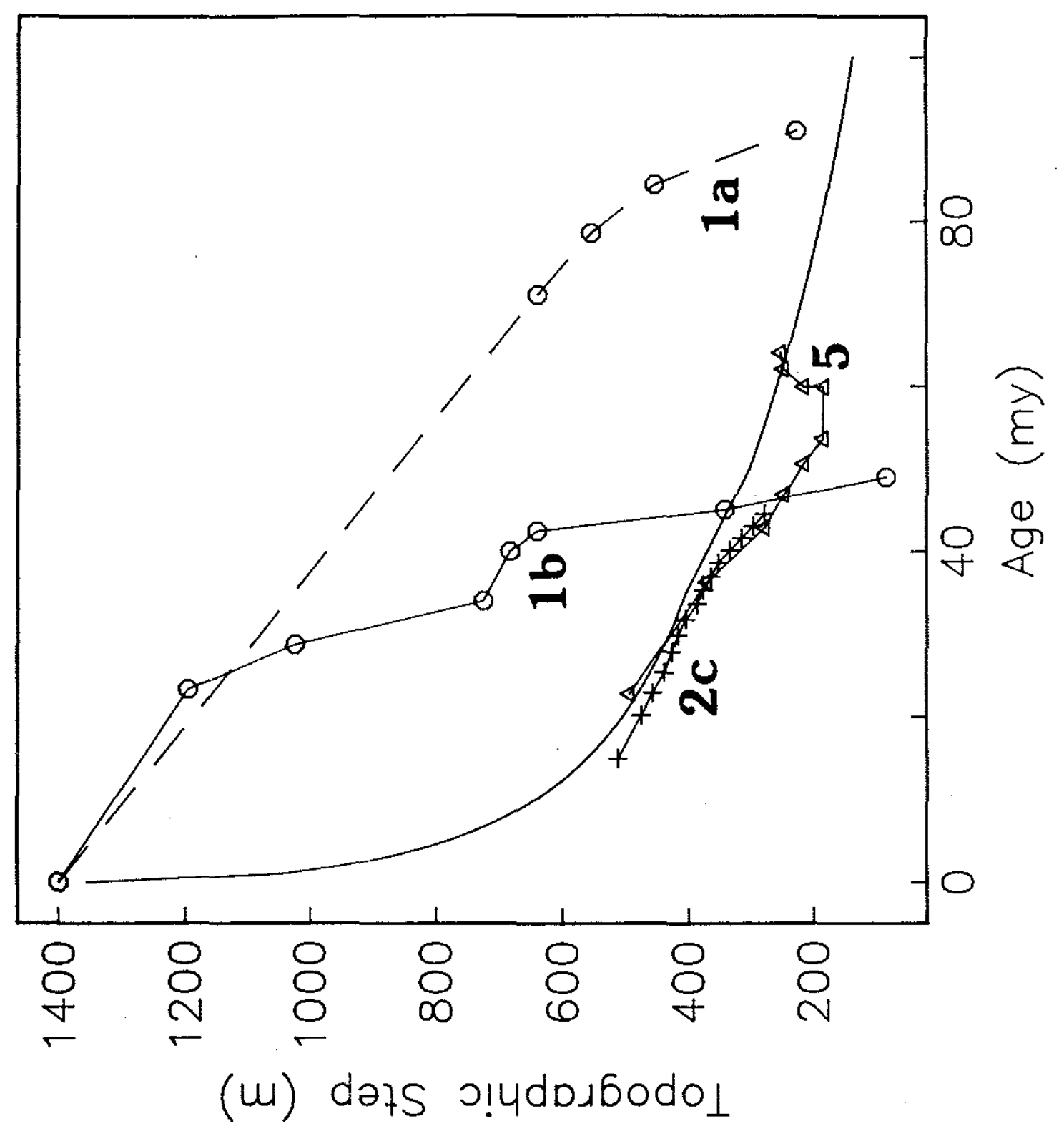


Chapter 5: INSTABILITIES IN THE COOLING OCEANIC PLATES

\subsection{Introduction}

Conductive cooling of the oceanic plates can explain the depth-age relationship until ages near and greater than $70 \mathrm{~m} \cdot \mathrm{y} \cdot$ (Parsons and Sclater, 1977). At $70 \mathrm{~m} \cdot \mathrm{y} \cdot$, the depthage curve flattens, probably reflecting an increased heat transport over and above that produced by conduction to the base of the lithosphere (McKenzie, 1967). This extra heat supply implies the existence of an advective mechanism which transfers heat from the mantle to the surface plate. Many explanations have been put forward to explain the flattening of the subsidence data that involve advection (McKenzie, 1967; Schubert et al., 1976; Parsons and Sclater, 1977; Parmentier and Turcotte, 1977; Parsons and McKenzie, 1978; Heestand and Crough, 1981; Jarvis and Peltier, 1982; Fleitout and Yuen, 1984a). However, recent observations of small scale (150-500 km in wavelength) geoid anomalies in the Central Pacific (Haxby and Weissel, 1986) and Central Indian oceans (Cazenave et al., 1987) have renewed interest in the explanations of the depth-age relationships that involve small scale (150-250 $\mathrm{km}$ wavelength) convective instabilities underneath the cooling plates.

Parsons and McKenzie (1978) showed that an oceanic plate can become convectively unstable as it cools. They divided the thermal plate into a rigid layer and a ductile layer where the boundary between the layers is controlled by 
the temperature. They then defined a local Rayleigh number, $\mathrm{Ra}_{\mathrm{b}}$, in the ductile portion of the boundary layer:

$$
R a_{b}=g_{0} \alpha \Delta T_{b} d_{b} 3 / k \mu_{b}
$$

where $g_{0}$ is the surface acceleration of gravity, $\boldsymbol{\alpha}$ is the coefficient of thermal expansion, $\boldsymbol{k}$ is the thermal diffusivity, and $\Delta \mathrm{T}_{\mathrm{b}}$ is the temperature difference, $d_{\mathrm{b}}$ is the thickness and $\mu_{\mathrm{b}}$ is the viscosity of the ductile portion of the thermal boundary layer. Since conductive cooling of the oceanic plates follows an error function temperature solution:

$$
d_{b} \propto \sqrt{k t}
$$

one can calculate the local Rayleigh number for the boundary layer. If this Rayleigh number exceeds a critical Rayleigh number Racr, the lower portions of the plate will convect, increasing the heat flux into the base of the plate. Parsons and McKenzie (1978) demonstrated that, with appropriate values for the thermal constants in the upper mantle but with a viscosity which is one order of magnitude less than the viscosity that is consistent with post-glacial rebound, the oceanic plates would go unstable near a plate age of $70 \mathrm{~m} \cdot \mathrm{y}$. . In a study of the onset of convective flow beneath a conducting lid, Houseman and McKenzie (1982) showed that the heat flux from convective instabilities can produce the observed depth-age relationship in the oceans. Yuen et al. (1981) also analyzed the local convective stability of a surface boundary layer due to large scale convective circulations in the mantle. They used a frozen- 
time eigenvalue analysis and assumed that the viscosity depends only on temperature. They found that the large viscosity of the cold thermal boundary layer at the surface exerts a stabilizing influence on the formation of convective instabilities. Yuen et al. (1981) also found that convective instabilities will grow either if the viscosity of the upper mantle is one order of magnitude less than the value which is consistent with post-glacial rebound data $\left(10^{20} \mathrm{~Pa} . \mathrm{s}\right)$ or if there exists a low viscosity zone in the uppermost part of the mantle with a viscosity of $10^{20}$ Pa.s. Jaupart and Parsons (1985) also concluded that, to produce convective instabilities within the lifetime of the oceanic plates, the viscosity of the uppermost mantle would need to be, at most, $10^{21} \mathrm{~Pa} . \mathrm{s}$.

Yuen and Fleitout (1984), Fleitout and Yuen (1984a) and Fleitout and Yuen (1984b) have studied the effect of temperature and pressure on the viscosity structure of the mantle and the development of convective instabilities at the base of the lithosphere. They found that the temperature and pressure dependence creates a low viscosity zone in the uppermost mantle beneath the lithosphere, with viscosities that are one to four orders of magnitude less than $10^{21} \mathrm{~Pa} . \mathrm{s}$. They also found that, in this region, convective instabilities could have fast growth rates.

Recent analyses of the SEASAT altimeter measurements of the geoid in the Central Pacific ocean (Haxby and Weissel, 1986) and the Central Indian ocean (Cazenave et al., 1987) 
have revealed a small wavelength $(150-500 \mathrm{~km})$ geoid signal which is $0.50-0.80 \mathrm{~m}$ in magnitude. These geoid anomalies are evident over seafloor of 5-10 m.y. in age and extend out to ages greater than $30-40 \mathrm{~m} . \mathrm{y} .$, with a possible trend to longer wavelengths at older ages. The signal is correlated across satellite tracks which are 80-120 km apart, so that the anomalies appear as long rolls which stretch in the direction of absolute plate motion. In particular, the direction of lineation is oblique to the Pacific-Farallon fracture zone traces, so the anomalies can not be due to ridge processes. Haxby and Weissel (1986) interpreted this signal as the direct reflection of small scale convection in the uppermost mantle, confined to a layer near $100 \mathrm{~km}$ thick. In order to produce an onset in the convective flow at 5-10 m.y., they postulated that the viscosity in this layer is three orders of magnitude below post-glacial rebound values. Buck and Parmentier (1986) analyzed the onset and development of convective instabilities in the upper mantle with a finite difference, two-dimensional fluid model. They used a relationship for the dynamic viscosity which depended on both temperature and pressure. Buck and Parmentier found that the convective flow could produce the observed magnitude and wavelengths of the SEASAT geoid anomalies, but not the persistence of the anomalies to older ages, in some. cases to ages greater than $50 \mathrm{~m} . \mathrm{y}$.. They explained the long life of the anomalies as evidence that the early instability had been "frozen in" to the elastic lithosphere. They also 
found that this convective flow which begins at young ages, does not perturb the mean depth-age relationship from the squareroot of age dependence of the conductive cooling solution, so that it cannot produce flattening at ages near $70 \mathrm{~m} \cdot \mathrm{y} \cdot \mathrm{.}$

Although the temperature and pressure dependence of the viscosity produces a low viscosity zone in the shallowmost mantle, the low viscosity zone thickens and decreases in magnitude with age. Its effects are therefore diminished with increasing age, reducing the small scale convective flow and its effect on the depth-age relationship. However, small degrees of melt have also been shown to decrease the viscosity of mantle material (Cooper and Kohlstedt, 1984). Since the presence of melt is thought to be confined to the top $200 \mathrm{~km}$ of the mantle (Anderson and Sammis, 1970; McKenzie, 1982), the low viscosity zone may be enhanced above $200 \mathrm{~km}$ and may persist to greater ages than predicted when just the effects of the temperature and pressure variations are considered. In this paper, we have studied the effects of a low viscosity zone in the uppermost mantle on instabilities in the cooling oceanic plates.

We have approximated the viscosity structure in the upper mantle by three layers consisting of a conducting lid, overlying the low viscosity zone which in turn overlies a constant viscosity layer extending to the base of the upper mantle. Using a finite element numerical method, we study the effect of the low viscosities, the layer thicknesses and 
the overall Rayleigh number on the convective instabilities and the surface anomalies which reflect them. We explore the effect of these parameters on the convective flow and its surface geoid, gravity and topographic expression.

We have also found that the low viscosity zone enhances the development of convective instabilities in the uppermost mantle. At reasonable viscosity contrasts the flow is first confined to the low viscosity zone, but eventually the horizontal temperature gradients at the base of the low viscosity zone will cause the bottom layer to go unstable as well. The time at which the first instability occurs depends upon the viscosity in the top layer, and the period for which it is confined to the low viscosity zone depends on the viscosity contrast between the layers. Moreover, the low viscosity zone reduces, but does not negate, the effect of the temperature anomalies due to convection in the bottom layer and in the lower part of the low viscosity zone, on the surface geoid, gravity and topography fields.

For reasonable values of the viscosities and viscosity contrast, the model produces the observed small scale geoid and topography anomalies. However, it cannot reproduce both the magnitude and the onset time of the observables with the same model. This limitation of the method is expected given the inherent limitations of a two-dimensional model in predicting a three-dimensional onset time, since we cannot model the effect of perturbations from three-dimensional flows or advection in and out of the plane of the 
calculation. Also, since we do not include the effects of any perturbations and since we use a conductive lid with a constant thickness which is too thick at young ages, we underestimate the onset time in the Earth. Therefore, we do not use the onset time as a constraint. We also calculate the mean depth-age, geoid-age and heat flow-age relationships from the flow. We find that the initial small scale flow causes these relationships to take on slightly shallower values but does not alter the squareroot of age dependence. However, when convection extends throughout the whole layer, the mean depth-age, geoid-age and heat flow-age relationships flatten. Therefore, the apparent conflict presented by the observations that small scale convection starts at near 5-10 m.y. in the Pacific but that the flattening in the depth-age relationship does not occur until $70 \mathrm{~m} \cdot \mathrm{y}$. can be resolved. Furthermore, the model predicts that the heat flow-age curve flattens earlier and reaches higher values at large ages than the plate model; And, recent measurements of heat flow on old seafloor in the Atlantic ocean support this early departure time and higher asymptotic value for the heat flow-age curve (Davis et al., 1984; Detrick et al., 1986; Louden et al., 1987).

\subsection{The Numerical Model}

We have nondimensionalized the equations of motion, heat transport and state through the following transformations: 


$$
\begin{aligned}
\mu^{\prime} & =\mu_{0} \mu \\
\left(x^{\prime}, z^{\prime}\right) & =d(x, z) \\
T^{\prime} & =\Delta T T \\
t^{\prime} & =\left(d^{2} / x\right) t \\
\rho^{\prime} & =\rho_{O} \rho
\end{aligned}
$$

where primes denote dimensional quantities and $\mu_{0}$ is the viscosity of the bottom layer, $x$ and $z$ are the horizontal and vertical coordinates respectively, $d$ is the depth of the convecting layer, $\rho_{0}$ is the reference density and $\Delta \mathrm{T}$ is the initial temperature contrast between the bottom and top boundaries. We used the values for the physical constants given in Table 5.1 .

After this transformation, the equation of motion, the heat equation and the equation of state are given by,

$$
\begin{gathered}
\nabla \cdot \sigma=-\mathrm{R}\left(\mathrm{T}-\mathrm{T}_{O}\right) \hat{z} \\
\partial \mathrm{T} / \partial \mathrm{t}+\mathrm{u} \cdot \nabla \mathrm{T}=\nabla^{2} \mathrm{~T} \\
1-\alpha \Delta \mathrm{T}\left(\mathrm{T}-\mathrm{T}_{O}\right)=\rho
\end{gathered}
$$

where $u$ is the velocity vector, $\hat{z}$ is a vertical unit vector, $\sigma$ is the stress tensor given by:

$$
\sigma_{i, j}=-p \delta_{i j}+\mu\left(\partial u_{i} / \partial x_{j}+\partial u_{j} / \partial x_{i}\right)
$$

where $\mathrm{p}$ is the pressure, and $\mathrm{R}$ is the Rayleigh number:

$$
R=g_{0} \alpha \Delta \mathrm{Td} d^{3} / \mu_{0} \kappa
$$

We solved these equations using a velocity based finite element method, which has been modified to solve the heat equation on finite difference grid, and followed the flow in time with an explicit time stepping method (Daly and Raefsky, 1985). Although the incompressibility equation: 


$$
\nabla \cdot \mathrm{u}=0
$$

is never explicitly solved, a penalty function treatment of the pressure forces incompressibility (Hughes et al., 1979). We tested the resolution of each run by repeating the calculation on a larger grid, usually double the size, and comparing the results. We also looked at enlarged portions of the temperature field in detail to insure that the contours were smooth and that no spurious values had entered the calculation.

All of the convection calculations began with the same idealized initial temperature structure and were subject to the same boundary conditions:

$$
\begin{aligned}
\mathrm{w} & =0, \quad \mathrm{z}=0,1, \mathrm{~b} \\
\mathrm{u} & =0, \quad \mathrm{z}=1, \mathrm{~b} \quad \mathrm{x}=0, \mathrm{~h} \\
\sigma_{\mathrm{xz}} & =0, \quad \mathrm{z}=0 \quad \mathrm{x}=0, \mathrm{~h} \\
\mathrm{~T} & =0, \quad \mathrm{z}=\mathrm{b} \\
\partial \mathrm{T} / \partial \mathrm{x} & =0, \quad \mathrm{x}=0, \mathrm{~h} \\
\partial \mathrm{T} / \partial \mathrm{z} & =0, \quad \mathrm{z}=0 \\
\mathrm{~T} & =1, \quad \mathrm{z}<\mathrm{b}, \quad \mathrm{t}=0
\end{aligned}
$$

where $u$ is the horizontal velocity and $w$ is the vertical velocity. These boundary conditions are also summarized in Figure 5.1.

We experimented with a number of box widths. Since all of the fluid will eventually convect, a compromise had to be found between the resolution of the flow (since there were limits on the total number of mesh points) and the disturbances due to the sidewalls. To test the effects of 
the sidewalls on our results, we ran a number of calculations on a series of grids with different aspect ratios: $8-$ by $-1(8 \times 1), 6 \times 1,4 \times 1$ and $2 \times 1$ boxes. In all of the calculations in our parameter suite, except for those at very low Rayleigh numbers and viscosity contrasts, we found that the $8 \times 1,6 \times 1$ and $4 \times 1$ boxes gave similar results within two or three percent in the mean flux. We chose a box length of $4 \times 1$ for most of our runs, therefore. However, because, at high Rayleigh numbers and viscosity contrasts, the grid must be very dense, some of the runs and many of the resolution calculations were run on a $2 \times 1$ grid.

To obtain this simplified model, a number of assumptions have been made. The first was to approximate the mechanical portion of the lithosphere by a conducting lid (Jaupart and Parsons, 1985). Since we could not incorporate any growth of the lid with time, its thickness had to be an average of the appropriate thickness at all ages. As a result the lid was too thick at young ages. To investigate the effect of the lid on the flow and it surface anomalies, we ran calculations for a number of lid thicknesses, $0 \mathrm{~km}, 25 \mathrm{~km}$ and $50 \mathrm{~km} \mathrm{thick}$, and found that the form of the instabilities and the ensuing flow did not vary appreciably. As expected the onset of the instability was delayed by a thicker $1 i d$, and the surface anomalies reduced in amplitude. We will discuss the runs in which we have varied the conducting lid thickness in more detail in the following sections, but we chose a conducting lid thickness 
of $50 \mathrm{~km}$ for the bulk of the parameter study, corresponding to a $30-50 \mathrm{~m} \cdot \mathrm{y}$. old plate.

Because we concentrated on the flow due to convective instabilities instigated by conductive cooling at the top of the layer, we neglected internal heating and heating from below. Since the upper mantle is believed to have a low concentration of radioactive elements, whose effects are in any case limited on the short time scales on which these processes are important, we can ignore internal heating (McKenzie and Richter, 1981). The effects due to heating from below are more difficult to assess. Since the instabilities in the top layer develop very quickly, they may reach the bottom layer before it goes unstable. Then, heating from below will only reinforce the flow due to the instabilities at the surface. At large Rayleigh numbers, the effects of instabilities in the bottom boundary layer must nevertheless be taken into account and, most certainly at large times, the effect of heating from below on the magnitude of the temperature difference across the fluid layers must be considered.

The third simplification is that our model is twodimensional and we have ignored the effects of advection in and out of the plane of the calculation. As a first approximation, however, a two dimensional model is adequate. Such an advective flow has been modelled in the laboratory by Parsons and Richter (1975), in a fluid tank with a top boundary which is rigid and moving with constant velocity. 
These experiments show that convective instabilities in a fluid with such a rigid moving top boundary will align themselves in the direction of motion of the boundary and that a two dimensional description of the flow is useful. The fourth assumption is in our choice for the physical parameters used in the scaling constants from the best estimates in the literature. These parameters are not always well known, especially at depths where convection is important. Since the small scale instabilities occur predominantly in the uppermost part of the mantle, we have chosen values for the constants which agree primarily with surface data (Table 5.1). The notable exception is the value for $\boldsymbol{\kappa}$, the thermal diffusivity. Since the thermal diffusivity only enters into the scaling of time and since the onset times are controlled by the material in the fluid upper mantle, we chose a value that is appropriate for the uppermost mantle.

A final consideration is the effect of the sidewalls. In our calculations due to the velocity boundary conditions on the sidewalls, the flow must either center on an upwelling or downwelling at a sidewall. Therefore, our estimates of the wavelengths of the convection are only approximately the wavelengths expected in the mantle. It is unclear whether the Earth has features that act in a way similar to sidewalls. Fracture zones and continental shelves might exert such an influence but, for a comparison 
to data, we will try to omit the features in the flow and in the surface anomalies produced by the sidewalls.

With this viscosity model of the upper mantle, consisting of a conducting lid, a low viscosity zone and a constant viscosity layer extending to the base of the upper mantle, we explored the effect on the convective flow of (1) low viscosities in the upper layer, (2) the Rayleigh number of the fluid layer, based on the viscosity of the bottom layer, (3) the relative thicknesses of the two fluid layers, and (4) the thickness of the conducting lid. In Table 5.2, we list the parameters for the runs that we shall present.

\subsection{Convection Induced by cooling from Above}

Four suites of calculations are presented (see Table 5.2). In the first suite, we varied the viscosity contrast while keeping the other parameters constant. For the second suite, we changed both the Rayleigh number based on the viscosity of the bottom layer and the Rayleigh number based on the viscosity of the top layer. In the third suite, we varied the relative thicknesses of the two fluid layers. Finally, for the fourth suite, we changed the thickness of the conducting lid.

In Figures $5.2,5.3$ and 5.4 , we have drawn the temperature structures for three calculations in which we have held the Rayleigh number at $10^{5}$, the layer thickness at $0.21(125 \mathrm{~km})$ and the conducting lid thickness at $0.087(50$ $\mathrm{km})$, while varying the viscosity contrast from 1.0 to 0.01 . 
In Figure 5.2, we present the temperature structures for no viscosity contrast (run 1 in Table 5.2) at $720 \mathrm{~m} \cdot \mathrm{y}$. and 770 m.y.. We defined the onset time in these calculations as the time at which the kinetic energy (the sum of the square of the velocities at each node in the numerical grid) exponentially increases due to the increase in the convective velocities. The onset time for this flow is at $700 \mathrm{~m} . \mathrm{y} .$, so these frames represent the flow $20 \mathrm{~m} \cdot \mathrm{y}$. and 70 m.y., respectively, after its onset. We defined the wavelength of the flow as the distance between the centers of adjacent downgoing plumes. Although the plumes are slightly irregularly spaced, the flow can be characterized by an $800 \mathrm{~km}$ wavelength. The horizontal temperature difference across the plume reaches a peak of $700^{\circ} \mathrm{C}$ but, by $770 \mathrm{~m} . \mathrm{y} .$, it has decreased to $450^{\circ} \mathrm{C}$ and, by $800 \mathrm{~m} \cdot \mathrm{y}$. , it has decreased to $250^{\circ} \mathrm{C}$.

In Figure 5.3, we have drawn the temperature structure for the second run in the first suite of calculations which has a viscosity contrast of 0.1 (run 2). The onset time for this flow is at $380 \mathrm{~m} \cdot \mathrm{y}$. and we have presented the temperature structures for this run at $400 \mathrm{~m} . \mathrm{y}$. and 450 m.y.. These frames are $20 \mathrm{~m} \cdot \mathrm{y}$. and $70 \mathrm{~m} \cdot \mathrm{y}$. , after the onset of the flow, respectively, which are the same intervals as presented for run 1. The sidewalls are again sites of downwelling, but the characteristic wavelength of the flow $(800 \mathrm{~km})$ is more regular than in the case with no viscosity contrast. Because the local Rayleigh number in the low 
viscosity zone has increased, the top boundary layer inside the low viscosity zone is thinner than for the case of no viscosity contrast. The magnitude of the temperature contrast across the plume reaches nearly $750{ }^{\circ} \mathrm{C}$ but, by 450 m.Y., it has decreased to $240^{\circ} \mathrm{C}$. Therefore, although the initial instability has the same magnitude as in the previous calculation with no viscosity contrast, it decreases more quickly.

With the same model geometry but a viscosity contrast of two orders of magnitude (run 3), the effect of the low viscosity zone dominates the flow. The effective Rayleigh number of the low viscosity zone, which is $9.26 \times 10^{4}$ (using the thickness and viscosity of the top layer, with $\Delta \mathrm{T}_{\mathrm{b}}$ equal to one), is near to the overall Rayleigh number of $10^{5}$ and the flow is initially confined to the low viscosity zone. The onset time for this flow is $85 \mathrm{~m} . \mathrm{y}$. and we have drawn the calculation at $91 \mathrm{~m} \cdot \mathrm{y} \cdot, 110 \mathrm{~m} \cdot \mathrm{y} \cdot$ and $130 \mathrm{~m} \cdot \mathrm{y} \cdot$ (Eigure 5.4). The wavelength of the small scale flow is 150 $\mathrm{km}$ and its magnitude reaches $450^{\circ} \mathrm{C}$ at $91 \mathrm{~m} . \mathrm{y}$. . By $6 \mathrm{~m} . \mathrm{y}$. later, however, the magnitude of the flow has decreased to $100{ }^{\circ} \mathrm{C}$. Since the short wavelength flow is uneven, as it cools beneath the top layer, it sets up longer wavelength temperature gradients which cause the bottom layer also to convect. This longer wavelength flow begins near $99 \mathrm{~m} \cdot \mathrm{y} \cdot$, and its contribution to the energy of the flow can be seen clearly in a plot of the kinetic energy with time (Figure $5.5(\mathrm{a}))$. By $110 \mathrm{~m} \cdot \mathrm{y} \cdot$ (Figure 5.4), this long wavelength 
convection can be seen in the temperature structure and its magnitude is close to $250^{\circ} \mathrm{C}$. At $130 \mathrm{~m} \cdot \mathrm{y} .$, the flow has become even more complicated. It is dominated by downwellings on either side of the box, with convective instabilities forming repeatedly in the top boundary layer. These instabilities are most often swept into the downgoing plume before initiating a significant flow of their own, and the downgoing plume exhibits these disturbances.

Since the lateral temperature variation is most important in calculating the geoid, topography, gravity and heat flow anomalies seen at the surface, we have presented it in detail here. However, the horizontal mean temperature structure is most important in the depth-age, geoid-age and heat flow-age relationships. In Figure $5.5(b)$, we have drawn the mean temperature structures for the last calculation (run 3 in Table 5.2) at $60 \mathrm{~m} . \mathrm{y} ., 91 \mathrm{~m} . \mathrm{y} \cdot, 110$ $\mathrm{m} \cdot \mathrm{y}$. and $130 \mathrm{~m} \cdot \mathrm{y}$. (see the figure caption for details). At $60 \mathrm{~m} . \mathrm{y} .$, the mean temperature structure reflects simple conductive cooling. By $91 \mathrm{~m} . \mathrm{y}_{\text {., }}$ a convective flow has formed in the low viscosity zone so that the mean temperatures are increased at the top of the layer and decreased at the bottom. At $100 \mathrm{~m} \cdot \mathrm{y} .$, longer wavelength convection has begun and the mean temperatures have been disturbed from their conductive cooling profile down to depths greater than $350 \mathrm{~km}$; and, by $130 \mathrm{~m} . \mathrm{y}$. , these temperature differences extend to the base of the box. Therefore, from these mean temperature structures, it is 
clear that the convective flow will alter the depth-age relationship from a conductive cooling curve (Houseman and McKenzie, 1982).

In the second suite of calculations in Figures 5.6 and 5.7, we have varied the Rayleigh number between $10^{4}$ and $10^{6}$, while holding the top layer thickness at 0.21 (125 km), the conducting lid thickness at $0.087(50 \mathrm{~km})$ and the viscosity contrast at 0.1 (runs 5 and 6 in Table 5.2). In Figure 5.6, we have drawn the temperature structure at $1750 \mathrm{~m} \cdot \mathrm{y}$. for a calculation at a Rayleigh number of $10^{4}$, where convection began at $1650 \mathrm{~m} . \mathrm{y}$. We have already discussed a calculation where the Rayleigh number is $10^{5}$, run 2 in Figure 5.3. In Figure 5.7, we have drawn the temperature structure at 89 m.y. in a $2 \times 1$ box, for a Rayleigh number of $10^{6}$. At this Rayleigh number, the effective Rayleigh number of the top layer is $9.26 \times 10^{4}$. Since the Rayleigh number for the whole layer is an order of magnitude higher than this effective Rayleigh number, however, a longer wavelength flow begins at nearly the same time. The small scale flow has a wavelength of $160 \mathrm{~km}$ and the longer wavelength flow superimposed on top of it has a wavelength of near $800 \mathrm{~km}$. The effect of increasing the Rayleigh number is very similar to increasing the viscosity contrast, except that the effective Rayleigh number of the low viscosity layer changes with that of the whole layer. The low viscosity zone is therefore not effective at confining the flow to the top layer at this viscosity contrast. 
We have also studied a set of calculations where we have kept constant a Rayleigh number based on the viscosity of the top layer. Runs 4, 11, 12 and 13 have a Rayleigh number of $3.2 \times 10^{7}$ based on the lower viscosity. Since the absolute viscosity of the top layer is then effectively constant, the onset time for the small scale convection is identical in these calculations. However, as the Rayleigh number based on the viscosity of the bottom layer decreases and the viscosity contrast increases, the onset of long wavelength convection is delayed (see Table 5.2).

In Eigures 5.8, we present calculations in which we have varied the thickness of the low viscosity zone, while holding the Rayleigh number at $10^{5}$, the conducting lid thickness at $0.087(50 \mathrm{~km})$ and the viscosity contrast at 0.1. In Figure 5.8(a), we have drawn the temperature structure at $520 \mathrm{~m} . \mathrm{y}$. for a calculation with a low viscosity zone which is $0.087(50 \mathrm{~km}$ ) thick (run 7 in Table 5.2). The onset time for this flow is $465 \mathrm{~m} . \mathrm{y} .$, and we have drawn the flow $55 \mathrm{~m} \cdot \mathrm{y}$. later. The characteristic wavelength of the flow has been reduced by the low viscosity zone to $600 \mathrm{~km}$. In Figure $5.8(\mathrm{~b})$, we have drawn the temperature structure at $270 \mathrm{~m} \cdot \mathrm{y}$. for a calculation with the top layer thickness at $300 \mathrm{~km}$ (run 8 in Table 5.2). The onset time for this flow is $250 \mathrm{~m} . \mathrm{y}$. for the convective flow which is confined to the low viscosity zone. It has a wavelength of $370 \mathrm{~km}$ at 250 m.y., but quickly decays into a longer wavelength flow commencing at $285 \mathrm{~m} . \mathrm{y}$. . Therefore, if the effective 
Rayleigh number of the top layer is large enough so that small scale convection begins, the depth of the low viscosity zone controls the wavelength of the shallow convective cells. However, the small scale flow will eventually decay into a longer wavelength flow, where the depth of the total fluid layer and the side boundaries control the wavelength of the flow.

In the final set of calculations, we have varied the conducting lid thickness to $25 \mathrm{~km}$ and $0 \mathrm{~km}$, while holding the Rayleigh number at $10^{5}$, the low viscosity zone thickness at $0.21(125 \mathrm{~km})$ and the viscosity contrast at 0.01 . In Figure $5.9(a)$, we have drawn the temperature structure at 58 $\mathrm{m} \cdot \mathrm{y}$. for the run with a $25 \mathrm{~km}$ thick lid and, in Figure $5.9(\mathrm{~b})$, we have drawn it at $41 \mathrm{~m} \cdot \mathrm{y}$. for a $0 \mathrm{~km}$ thick lid. The characteristic wavelengths of these flows are the same at $150 \mathrm{~km}$, but their onset times, the times at which they develop longer wavelength flows and their magnitudes are different. The onset times for runs with a $50 \mathrm{~km}, 25 \mathrm{~km}$ and $0 \mathrm{~km}$ lid are, respectively, $85 \mathrm{~m} . \mathrm{y} ., 54 \mathrm{~m} . \mathrm{y}$. and $37 \mathrm{~m} . \mathrm{y} .$, and they develop the longer wavelength $(1200 \mathrm{~km})$ flows 14 $\mathrm{m} \cdot \mathrm{y} ., 11 \mathrm{~m} \cdot \mathrm{y}$. and $7 \mathrm{~m} \cdot \mathrm{y}$. later. Therefore, the conducting lid delays the instigation of a small scale convective flow and its development into a longer wavelength flow.

In Figure 5.10, we plotted the initial onset times, of. flow within either of the fluid layers versus the viscosity contrast at different Rayleigh numbers (based on the viscosity of the bottom layer), for calculations with a low 
viscosity zone thickness of $0.21(125 \mathrm{~km})$ and a conducting lid thickness of $0.087(50 \mathrm{~km})$. From the simple boundary layer argument given in the introduction, with $\Delta \mathrm{T}$ fixed, the onset time is related to the viscosity in the layer by: $\log t \alpha s \log \mu$

with $S=2 / 3$. In our suite of calculations, $S$ is not constant but is close to $1 / 2$. The failure of the boundary layer argument reflects the transition in the flow from convection that fills both fluid layers at low viscosity contrasts to small scale convection at large viscosity contrasts. From this simple boundary layer argument, one can also derive a relationship between the Rayleigh number and the onset time, which gives a log-log slope of $-2 / 3$. For the second suite of calculations in which we vary only the Rayleigh number at a viscosity contrast of 0.1 , the onset times do not closely follow this relationship as well, especially at a Rayleigh number of $10^{6}$ where the small scale and longer scale convection begin at near the same time.

In summary, we have found that the magnitude and onset time of the flow is very sensitive to the viscosity structure. In particular, the initial onset time depends on the absolute viscosity of the top layer. If the viscosity contrast is large enough so that the local Rayleigh number in the top layer is comparable to the Rayleigh number for the whole fluid layer, then the flow will remain in the low viscosity zone for a period, but will eventually decay into 
a longer wavelength flow extending throughout both fluid layers.

\subsection{Geoid, Topography, Gravity and Heat Flow}

To calculate the geoid, gravity and topography anomalies from these temperature structures, we have used the Green's function method described by Parsons and Daly (1983). The temperature field is decomposed into its Fourier components and, at each wavenumber, the Green's function response to the temperature anomalies at depth, the kernel, is calculated for each observable. The surface topography kernel represents the effect of a density anomaly, at depth $\mathrm{z}$, on the surface topography through the transmission of normal stress. We have also included the effect of a $10 \mathrm{~km}$ elastic plate into the surface topography kernel (Detrick et al., 1986), which is a lower bound on the elastic plate thickness for a $30-50 \mathrm{~m} \cdot \mathrm{y}$. old plate (Watts, 1978). The gravity kernel includes the effect of both the topography on the boundaries and the density variations in the layer. Finally, the geoid kernel can be derived from the gravity kernel through Brun's formula:

$$
N^{\prime}(k)=g^{\prime}(k) / k^{\prime} g_{0}=\left(d / g_{0}\right) g^{\prime}(k) / k
$$

where $\mathrm{N}$ is the geoid anomaly, $g_{0}$ is the acceleration of gravity and $\mathrm{k}$ is the wavenumber. The components of the temperature field are weighted by the kernels at each depth and integrated over the depth, and the resulting onedimensional array is Fourier transformed back to produce the 
anomaly. The application of the method to this model is discussed in detail in Robinson et al. (1987a; Chapter 2). The heat flow, $q$, at the surface of the model can be written:

$$
\mathrm{q}^{\prime}=\mathrm{k}_{\mathrm{C}} \Delta \mathrm{T}_{\mathrm{i}}^{\prime} / \Delta \mathrm{z}_{\mathrm{i}}^{\prime}
$$

where $\mathrm{k}_{\mathrm{C}}$ is the thermal conductivity. We calculate $\Delta \mathrm{T}_{1}$ and $\Delta z_{1}$ by differencing the temperature values and depths, respectively, for the top two rows of nodes.

In Figure 5.11, we have drawn the geoid, topography, gravity and heat flow anomalies at $400 \mathrm{~m} \cdot \mathrm{y}$. for run 2 (see Table 5.2). Each of the anomalies are elevated above the upwellings and depressed above the downwellings. Convection has begun in the layer at an $800 \mathrm{~km}$ wavelength and these anomalies exhibit that wavelength as well. Initially we estimated the amplitudes of the different wavelength anomalies with a Fourier decomposition of the total anomaly. We found, however, the estimates taken by eye were very close to those given by the Fourier analysis, and we have since relied on the estimated values. The geoid anomaly in this run is very large reaching a magnitude of near 14 meters, and the topography anomaly is similarly large at 3.3 $\mathrm{km}$. The gravity and heat flow anomalies reach a magnitude of $110 \mathrm{mgals}$ and $1.3 \mathrm{mw} / \mathrm{m}^{2}$, respectively. These anomalies represent the flow $20 \mathrm{~m} \cdot \mathrm{y}$. after its onset. By $50 \mathrm{~m} \cdot \mathrm{y}$. after the onset, the geoid, topography, gravity and heat flow anomalies have reached magnitudes of about $3 \mathrm{~m}, 1.2 \mathrm{~km}$, $35 \mathrm{mgals}$ and $12 \mathrm{mw} / \mathrm{m}^{2}$. The geoid and gravity anomalies 
reach their maximum amplitudes quickly, whereas the heat flow anomaly is delayed by the time it takes for the perturbed temperatures to diffuse into the lid.

As we increase the viscosity contrast, however, the magnitude of the surface anomalies decrease. In Figure 5.12, we have drawn the surface anomalies for run 3 which has the same geometry as the previous run, but a viscosity contrast of 0.01 . In this figure, we have included two time slices, at 91 and $110 \mathrm{~m} \cdot \mathrm{y}$. . The onset time is $85 \mathrm{~m} \cdot \mathrm{y}$. for the small scale convection in this run, so that, by $91 \mathrm{~m} \cdot \mathrm{y}$. , the small scale convection is already established. The anomalies reflect the $150 \mathrm{~km}$ wavelength of the convective flow, but also exhibit some long wavelength behavior. The magnitude of the small wavelength anomalies averages about $40 \mathrm{~cm}$ for the geoid, $250 \mathrm{~m}$ for the topography, 15 mgals for the gravity and $0.07 \mathrm{mw} / \mathrm{m}^{2}$ for the heat flow. These magnitudes persist until the onset of the longer wavelength convection. However, these values have decreased by over an order of magnitude from the previous case. The long wavelength convective flow is evident in the kinetic energy by $99 \mathrm{~m} \cdot \mathrm{y}$. and, by $110 \mathrm{~m} \cdot \mathrm{y} \cdot$, and the geoid anomaly reflects this longer wavelength first among the different observables. The appearence of longer wavelength anomalies in the heat flux is delayed due to the presence of the conducting lid. The magnitude of the longer wavelength signal reaches over $1.5 \mathrm{~m}$ in the geoid and $450 \mathrm{~m}$ in the 
topography. The small scale anomalies are superimposed on top of it, and their amplitudes are irregular.

Finally, in Figure 5.13, we have drawn the surface anomalies at $89 \mathrm{~m} \cdot \mathrm{y}$. for run 6 , where the viscosity structure is the same as in our first example but the Rayleigh number has been increased by an order of magnitude to $10^{6}$. The anomalies are again dominated by shorter wavelengths of near $150 \mathrm{~km}$. The small wavelength anomalies range from $20 \mathrm{~cm}$ to $60 \mathrm{~cm}$ in the geoid and $200 \mathrm{~m}$ to $400 \mathrm{~m}$ in the topography. These values persist until the longer wavelength convection is fully developed and are comparable to those for the last case (run 2) that had a viscosity contrast of two orders of magnitude and a Rayleigh number of $10^{5}$.

Because of the variability with time, with distance across the box and with wavelength, it is very difficult to characterize the behavior of the anomalies with the parameters in the model and we can only draw some very broad conclusions. In Table 5.2, we have listed the onset times of the small and longer wavelength flows. Alongside these times, we have catalogued the times and the amplitudes at which the geoid and topography anomalies reach their maxima for each of these flows. In all of the cases that we examined, the geoid and topography anomalies reached their maxima at similar times, so that we list only one time for each flow. After reaching these maximum values, the small scale anomalies persist at that amplitude until the longer 
wavelength convection develops. In order to minimize the effects of the sidewalls, we excluded the portions of the profile $200 \mathrm{~km}$ from either sidewall.

As the viscosity contrast increases, the geoid and topography kernels decrease sharply in magnitude in the low viscosity zone. They therefore reduce the effect of the longer wavelength temperature anomalies on the geoid and topography anomalies. Also, as mentioned above, the geoid kernels become negative at depth for viscosity contrasts near and above two orders of margnitude which counteracts the positive contributions from shallower depths. The geoid anomalies, therefore, decrease faster than the topography anomalies as the viscosity contrast increases (Figure 5.12). These effects can be seen in the first suite of calculations where we have changed only the viscosity contrast (Table 5.2)

In the suite of calculations where we have kept the viscosity in the top layer constant (runs 4, 11, 12 and 13) the magnitude of the small scale convective anomalies is similar. As the viscosity in the bottom layer increases, the onset of longer wavelength convection is delayed but the temperature anomalies are increased. However, since the viscosity contrast increases as the viscosity in the bottom layer increases, the change in the kernels with the viscosity contrast counteracts the increasing temperature anomalies, so that the geoid and topography anomalies decrease overall. 


\subsection{Depth-Age, Geoid-Age and Heat Flow-Age}

In the plate model, the mean depth and geoid values are given by integrals over the thermal plate thickness of the mean temperature profile, with unit weighting for the depth integral and with a weighting of $z$ (representing depth in the model) for the geoid integral (Haxby and Turcotte, 1978). However, these weightings and the cutoff point at the base of the thermal plate are based on assumptions, where the most important is that convective temperature anomalies immediately beneath the thermal plate are not reflected in the mean depths and geoid values. The plate model, nevertheless, reproduces the observed depth-age relationship.

Houseman and McKenzie (1984), Fleitout and Yuen (1984a) and Buck and Parmentier (1986) extended this method of calculating the geoid and topography anomalies from the plate model to their calculations of the surface expression of convective anomalies. In a convective flow, however, the temperature variations are significant throughout the fluid. Extending the formalism of the plate model to convective calculations will, therefore, lead to errors in interpreting the observables. In our calculations, we use the Green's function method to obtain an approximate weighting and integrate the temperature structure down to the base of the fluid layer. 
The best calculation for the depth-age and geoid-age relationships given the results of our convection modelling would embed the temperature structures in a threedimensional viscosity structure representing the oceanic mantle and, with three-dimensional kernels, to calculate the mean depths with age. Unfortunately, this calculation is very difficult and, furthermore, it would be premature since the temperature calculations were only formulated on a twodimensional grid. Instead, since our calculations give the temperature structure at a specific age, we have approximated the above method by integrating the mean temperature structure at each age and weighting it with the kernel for the longest wavelength in the ocean basin. For the Pacific, this wavelength is near 10,000 and, for the North Atlantic, it is near 3,000. We have therefore chosen a wavelength, to approximate all of the ocean basins, of $4800 \mathrm{~km}$ which corresponds to $8 \mathrm{~d}$ in our model. The choice of the specific wavelength is not crucial, since at these long wavelengths the kernels do not change rapidly with wavelength.

We have drawn the kernel for the geoid and topography anomalies at a $4800 \mathrm{~km}$ wavelength for viscosity contrasts between 1.0 and 0.001 in Figure 5.16. At this wavelength, the viscosity contrasts of 0.1 and 0.01 do not seriously effect the topography kernel, but a viscosity contrast of 0.001 depresses the kernel below the low viscosity zone to less than 30-40\% of its value with no viscosity contrast. 
The gravity kernels are more strongly affected by the viscosity contrast. By a viscosity contrast of 0.01 , the kernel has an overall magnitude of less than half of its value with no viscosity contrast. By a viscosity contrast of 0.001 , the kernel has become negative at depths in and below the low viscosity zone.

The behavior of the topography kernels with the addition of a low viscosity zone indicates why the simple weighting in the plate model, of unit value down to the base of the thermal plate and zero below, leads to the correct depth-age relationship. In Figure 5.14, the kernels have unit value in the conducting lid. In the low viscosity zone below the lid, if the viscosity contrast is large enough, the kernels fall to much lower values. This structure approximates that used in the thermal plate calculations, but is due to the influence of the low viscosity layer and not to the absence of strong temperature anomalies below the plate. The observation that the depth-age anomaly follows a thermal plate calculation is, therefore, a strong argument for the existence of a low viscosity zone at depth in the mantle.

In order to significantly effect the depth-age relationships, cooling must extend to a depth where the kernels downweight the contribution of the relatively cooler material in the bottom boundary layer of the convecting cells in comparison to the heated material in the top boundary layer. Before cooling extends to such depths, the 
heated and cooled materials, when integrated to form the depth-age relationship, cancel each other out and the result appears like conductive cooling. In the presence of small scale convection, since the kernels decrease with depth in the low viscosity zone, the relatively cooler bottom boundary layer of the small wavelength convection cells is somewhat downweighted in comparison with the top boundary layer. However, this effect is not significant until the non-dimensional viscosity in the top layer is equal to or greater than 0.001 , where the kernels reach $40 \%$ of their initial value by the bottom of the low viscosity zone.

In Figure 5.15, we have drawn the depth-age relationships for runs $1,2,3$ and 4 . These runs have a Rayleigh number of $10^{5}$, a low viscosity zone of $125 \mathrm{~km}$, a conducting lid thickness of $50 \mathrm{~km}$ and a viscosity contrast which ranges from 1.0 to 0.0032 . When the viscosity is 0.1 or less, since convection has not begun in the layer, the depth-age follows a curve proportional to the square root of age. Because the kernels are not the same as the depth weighting for the cooling halfspace model (i.e. are not one everywhere), this curve is not the curve for a cooling halfspace model, but it is linear with the square root of time. For a viscosity contrast of 0.01 or greater, small scale convection begins in the layer at young ages. In run 3, small scale convection begins at $85 \mathrm{~m} . \mathrm{y}$.. Since the kernels decrease in the low viscosity zone with depth, the depth-age curve is retarded slightly at times near those 
with the maximum temperature anomalies in the small scale flow, 90-95 m.y. (Table 5.2). Run 4 also is retarded after the onset of small scale convection.

However, when long wavelength convection begins in the layer, the decrease in the kernels with depth, as they.go to zero at the bottom boundary of the box, significantly downweights the relatively cooler bottom boundary layer of the convection cell. The depth-age relationship also begins to oscillate when the long wavelength convection becomes pronounced in the layer, as for run 3 after $120 \mathrm{~m} \cdot \mathrm{y}$. (figure 5.15). This oscillation resembles in detail the depth-age relationships calculated by Houseman and McKenzie (1982) of a model of convection underneath a conducting lid in a constant viscosity layer. They found that if the convection is initiated with a perturbation then the oscillations are damped and the depth-age curve flattens smoothly in agreement with the mean depth-age data. We do not include such a perturbation in our calculations, but it would have the same effect in this model, limiting the size of the initial departure from the conductive cooling depth-age curve and causing the depth-age curve to flatten as in the depth-age data (Houseman and McKenzie, 1982).

In Figure 5.16, we have drawn the depth-age relationships for the suite of calculations where the Rayleigh number based on the top layer viscosity has been held constant at $3.2 \times 10^{7}$. The onset times of small scale convection in these calculations are the same within $1 \mathrm{~m} . \mathrm{y}$. , 
so that the initial departure from the square root of age dependence, the conductive cooling solution, is at the same time. Since the kernels decrease faster in the low viscosity zone as the viscosity contrast increases, however, the departure is larger for the larger viscosity contrasts. As the Rayleigh number based on the viscosity of the bottom layer increases, the onset time of the long wavelength convection decreases and the large departure due to the onset of the long wavelength convection occurs earlier. For a Rayleigh number based on the bottom layer viscosity of $10^{6}$, the onset of the long wavelength convection occurs very close to the short wavelength convection.

The geoid-age relationship is also strongly affected by the behavior of gravity and geoid kernels with depth, except for the fact that the kernels can go negative with depth at high viscosity contrasts. Then the relatively cooler bottom boundary layer of the small wavelength anomalies is not downweighted but adds to the effect of the heated top boundary layer. Therefore, the separation of heat by the small scale convection in the top and bottom boundary layers of the convection cells is more pronounced in the geoid-age relationship.

In Figure 5.17, we have drawn the nondimensional geoidage relationships for runs $1,2,3$ and 4 . When the viscosity contrast is equal to or less than 0.1 , the geoidage curves follow that of a conductively cooling halfspace, which is proportional to age. When the viscosity contrast 
is 0.01 and small scale convection has begun in the low viscosity layer, since the geoid kernel is still positive everywhere, the geoid-age curve departs slightly from the halfspace solution but, after long wavelength convection begins, its departure is more pronounced. In the case of a viscosity contrast of 0.0032 , however, the geoid kernel has become negative inside the low viscosity zone and the run departs from linear behavior immediately after the onset of small scale convection. After the onset of long wavelength convection, the geoid-age relationship departs still further from the geoid-age relationship for a halfspace and is very time dependent. For this high viscosity contrast, therefore, the small scale convective temperature anomalies are seen in the geoid-age relationship whereas they have little effect on the depth-age relationship.

In Figure 5.18, we have drawn the geoid-age curves for the set of runs where the viscosity of the top layer has been held constant, and the Rayleigh number based on the viscosity of the top layer is $3.2 \times 10^{7}$. As in the last suite of calculations, the geoid-age curves depart much more dramatically for large viscosity contrasts than the depthage curve. At a viscosity contrast of 0.001 , in particular, the kernels are very negative, so that the geoid actually increases above its initial value at the ridge.

In Figure 5.19, we have drawn the heat flow-age relationships for runs $1,2,3$ and 4 . The heat flow-age relationship can be calculated by applying equation (5.13) 
to mean temperature profiles for various ages. Unlike the geoid and topography relationships, the heat flow-age relationship directly reflects the temperature structure in the conducting lid with time. As in the depth-age and geoid-age relationships, because convection has not yet started in the runs at a viscosity contrast of 1.0 and 0.1 , the heat flow-age relationship follows that of a cooling halfspace. At viscosity contrasts of 0.01 and 0.0032 , however, the heat flow-age relationship departs from the halfspace curve earlier than predicted by the plate model and soon after the onset of small scale convection. The time interval between the onset of convection at depth and its effect on the heat flow-age curve is close to the conductive time constant for a $50 \mathrm{~km}$ thick conducting lid of $7 \mathrm{~m} \cdot \mathrm{y}$. . The heat flow also further increases after the onset of long wavelength convection.

\subsection{Comparison to the observations}

We can compare the surface anomalies, that are produced by the cooling instabilities, to the observations of small scale geoid and topography anomalies and to the depth-age, geoid-age and heat flow-age relationships in the Pacific. Small scale anomalies have been observed in the SEASAT data sets in a number of oceanic regions, including the Central Pacific ocean and the Central Indian ocean (Haxby and Weissel, 1986; Cazenave et al., 1986). However, since a shipboard data set of gravity and topography, which shows 
the small scale anomalies, exists in the Southeast Pacific, we will perform our analysis in this region.

In the SEASAT data set over the Southeast Pacific, there is a signal that is lineated in the direction of plate motion which is oblique to the Pacific-Farallon fracture zone trends, of small undulations with a magnitude of 50-80 $\mathrm{cm}$ (Haxby and Weissel, 1986). Their wavelength ranges from 150-500 $\mathrm{km}$ with a possible trend to longer wavelengths with increasing age. The signal becomes apparent in the geoid field at a plate age of $5-10 \mathrm{~m} \cdot \mathrm{y}$. and persists to ages greater than 30-40 m.y.. Haxby and weissel (1986) interpret this signal as a reflection of small scale convection underneath the Pacific plate, in a low viscosity layer with a viscosity contrast that is three orders of magnitude below the estimate of the upper mantle viscosity based on postglacial rebound.

Since this small wavelength signal is near the limits of the resolution of the SEASAT data set, a shipboard study was undertaken to test its existence and to measure the topographic signal, if any, that accompanies it (Parsons et al., 1985). Seven long lines of gravity and topography data were collected: one in a region where no signal was seen by SEASAT and which was very close to the East Pacific Rise, three where the signal was clearly seen but close to its onset over $6 \mathrm{~m} . \mathrm{y}$. old seafloor, and three over much older 30-40 m.y. old seafloor. The study confirmed the SEASAT results by finding a $\sim 15$ mgal gravity signal at a wavelength 
of 150-250 km away from the ridge. The signal can also be correlated across the lines as in the SEASAT data. A $\sim 250 \mathrm{~m}$ topographic signal was also found which correlated with the gravity anomalies.

In these calculations the conducting lid thickness is too large to compare to the small scale anomalies observed on 5-40 m.y. old lithosphere in the Pacific. In particular, the reduction of the conducting lid thickness to $25 \mathrm{~km}$ increases the anomalies by $50 \%$ in the geoid and over $100 \%$ in the topography. Given this correction, the magnitude of these small scale surface anomalies are in the range that is observed (see Table 5.2, runs 2, 3, 4, 11 and 12). Their wavelength is constrained by the thickness of the low viscosity zone so that, to produce an initial wavelength of 150-250 km, a thickness of $75-125 \mathrm{~km}$ is required.

In our model with atop layer thickness of $125 \mathrm{~km}$, the predominant wavelengths are $150-250 \mathrm{~km}$ at young ages but then trend to longer wavelengths, as the bottom layer begins to convect. After the onset of long wavelength convection, the longer wavelength anomalies are superimposed on the shorter wavelength anomalies. As the temperature differences which drive the small scale flow decay, the smaller wavelength anomalies die out, and the longer wavelengths dominate. When the Rayleigh number is greater than $10^{6}$, the small wavelengths enter the observables at a young age and trend smoothly to longer wavelengths, which is in accordance with what is observed. Therefore, a Rayleigh 
number of $10^{6}$ or greater is favored by this data set. Furthermore, since an increase in the viscosity contrast further delays the onset of long wavelength convection, the persistence of the shorter wavelength signal for over 30 m.y. also indicates a large viscosity contrast at depth of at least 0.01 .

When the viscosity contrast is two orders of magnitude or greater at high Rayleigh numbers, the long wavelength amplitudes are similar to the amplitudes of the smaller wavelength anomalies (see Table 5.2). However, in this model, we have neglected heating from below which at large ages would strengthen the long wavelength anomalies, so that they would be visible as large geoid and topographic anomalies in the ocean basins. Since many long wavelength features are observed in the Pacific at large ages, most notably mid-plate swells, the data might not require small amplitudes in these long wavelength anomalies. In particular, since swells have the same shape as the longer wavelength anomalies in the model, the long wavelength signal would be indistinguishable from that of mid-plate swells (Robinson and Parsons, 1987; Chapter 3). Robinson and Parsons (1987) have applied this viscosity model to the case of upper mantle convection heated from below and found that the swells at Hawaii, Bermuda, the Marquesas and Cape Verde can be explained as the surface expression of these convecting cells with a viscosity contrast which is 0.4-0.01 in magnitude. An interpretation of the data in the Pacific 
is, therefore, that the breakdown of small scale convective flow underneath the plate is succeeded by the formation of longer wavelength features such as mid-plate swells.

One observation that we cannot interpret properly with our two-dimensional model is the onset time of the small scale convection. As mentioned above, in a threedimensional model, advection perpendicular to the plane would communicate the presence of temperature anomalies due to the instability at greater ages to the fluid under the younger plate. This shear and thermal coupling would then cause the boundary layer to go unstable at a younger age and at one that might be in agreement with the data.

In the depth-age relationship over the cooling instabilities, the small scale convection does not strongly affect the squareroot of age dependence, but the depths are slightly shallower. In the region of the central Pacific where the small scale anomalies are observed, the depths are also shallower with age then observed elsewhere in the Pacific. In the models, significant departures in the theoretical depth-age relationship occur only after the onset of the longer wavelength convection and, in the Pacific, the depth-age curve departs from that of a conductively cooling halfspace near $70 \mathrm{~m} . \mathrm{y}$.. Therefore, although this two-dimensional model cannot accurately predict the initial convective onset time, it predicts the general behavior of the depth-age curve. Furthermore, the departure times from the conductive cooling depth-age curve 
in the models with the parameters which fit the small scale anomalies are between 50 and $80 \mathrm{~m} \cdot \mathrm{y}$. (see Table 5.2). In particular, if run 12 , with a Rayleigh number of $3.2 \times 10^{5}$ and a viscosity contrast of 0.01 , were scaled so that the Rayleigh number was $10^{6}$, then the departure time would lie between 55-65 m.y.. However, if the low viscosity zone were due primarily to the temperature and pressure conditions in the mantle then the low viscosity zone would have decreased in magnitude from its initial value under young seafloor (Fleitout and Yuen, 1984a). Therefore, the viscosity contrast would be nearer 0.1 and would give a slightly retarded onset time for the long wavelength convection. This onset time would be no greater than for the calculation with a continuous viscosity contrast of 0.1 (run 6), so that the onset time is bounded above by $90 \mathrm{~m} . \mathrm{y} .$.

In the geoid-age relationship, if the viscosity contrast is small enough so that the kernels remain positive throughout the layer, then the departures from a squareroot of age dependence are at the same age as those observed in the depth-age relationship. However, if the viscosity contrast is large enough so that the kernels are negative at depth, then the geoid-age relationship will depart from the halfspace model after the onset of small scale convection which is much earlier than predicted in the depth-age relationship. Unfortunately little data exists to constrain the geoid-age relationship in the oceans except from the study of fracture zones which may strongly affected by the 
local mantle flow connected with the fracture zone (Craig and McKenzie, 1982; Robinson et al., 1987b; Chapter 4). Finally, in the heat flow-age relationship, the increased advection of heat due to the convection at depth affects the surface heat flow quickly after the onset of convection. Recent observations indicate that the heat flow-age relationship in the Atlantic does not follow a plate model at large ages. On old seafloor in the Atlantic, far away from swells or any other known thermal sources, the heat flow values are $50-53 \mathrm{mw} / \mathrm{m}^{2}$ whereas the plate model predicts values between 40 and $45 \mathrm{~mW} / \mathrm{m}^{2}$ (Davis et al., 1984; Detrick et al., 1986; Louden et al., 1987). These values point to either an earlier departure time from the conductive cooling curve than in the plate model (i.e. earlier than $120 \mathrm{m.y.)}$, or an increase in the heat flow with age. Because the conducting lid is $50 \mathrm{~km}$ thick, these calculations predict an earlier departure time than the plate model. For at least one of these calculations (run 3), the heat flow also rises at large ages. However, in all of the runs with a viscosity contrast which is greater than two orders of magnitude, the theoretical heat flow values at older ages are between 50 and $55 \mathrm{~mW} / \mathrm{m}^{2}$. These values agree with those observed in the West Atlantic (Detrick et al., 1986; Louden et al., 1987). At a Rayleigh number of $10^{6}$ and a viscosity contrast of 0.01 , the heat flow age curve falls between runs 3 and 4 in Figure 5.19, so that it is also in 
agreement with the measured heat flow values in the Atlantic.

In summary, with a Rayleigh number of $10^{6}$, a low viscosity zone thickness of $125 \mathrm{~km}$ and a viscosity contrast of 0.01 , the model predicts the magnitude and wavelength of the short wavelength anomalies seen in the SEASAT and shipboard data. It also produces flattening in the depthage relationship between 55 and $65 \mathrm{~m} . \mathrm{y}$.. Since a twodimensional model cannot approximate the correct threedimensional onset time, it cannot exactly reproduce the early onset times observed for small scale convection in the Pacific. Rather a model, which includes the shear and thermal coupling of the convective instability to the fluid under younger seafloor, is needed to constrain the onset time. The shear coupling would produce onset times very much younger than that predicted by boundary layer stability calculations and by two-dimensional convective flow calculations. We also cannot predict the exact values of the Rayleigh number and the viscosity contrast. Both of these parameters effect the local Rayleigh number in the low viscosity zone.

\subsection{Conclusions}

We have applied a finite element model to the study of convective instabilities in a conductive boundary layer, formed by cooling through the top surface. We compared our results to two sets of observations thought to reflect 
convective instabilities in the oceanic plates: (1) small scale geoid and topography anomalies in the Southeast Pacific and (2) the flattening of the mean depth-age and heat flow-age relationships.

Much evidence exists for a low viscosity zone in the uppermost mantle from seismic studies (Anderson and Sammis, 1970; Solomon, 1972; Forsyth, 1977; Weilandt and Knopoff, 1982), studies of the flow underneath fracture zones (Craig and McKenzie, 1986; Robinson et al., 1987b), and studies of the presence of melt in the upper mantle (Cooper and Kohlstedt, 1984; McKenzie, 1984). Most importantly, however, theoretical calculations of the viscosity structure with depth which is dependent on temperature and pressure predict a low viscosity zone (Fleitout and Yuen, 1984a; Buck and Parmentier, 1986).

In our model, we do not assume that the viscosity depends solely on temperature and pressure. Rather we suppose that a low viscosity zone retains its initial thickness out to large ages both for simplicity and to reflect the effect of the presence of melt above $200 \mathrm{~km}$ in depth. The small scale convection cells in this model therefore exhibit a small wavelength until long wavelength convection is instigated in the box, and the transition to longer wavelengths in the anomalies is more abrubt than in calculations where only the effects of temperature and pressure are considered. Since we include the effects on the surface anomalies of the convective temperature 
differences to the base of the box, the model produces a large departure from the depth-age curve for conductive cooling.

As we decrease the viscosity contrast between the layers and as we increase the Rayleigh number, the convective onset time decreases. The relationship between the onset time and the viscosity contrast in the calculations is nearly logarithmic with a. slope which is not constant, but is close to $1 / 2$. As the viscosity contrast becomes large enough so that the Rayleigh number for the low viscosity zone is greater than the critical Rayleigh number, small scale convection cells form which remain inside the low viscosity zone, and later decay into long wavelength convective anomalies which extend throughout the whole layer. The small scale convective cells persist for a time which increases as the viscosity contrast increases, but decreases as Rayleigh number of the two fluid layers increases (Table 5.2, runs 3 and 12).

Small scale anomalies have been seen in the geoid field of the Central Pacific, and are roughly $50-80 \mathrm{~cm}$ in magnitude with wavelengths ranging from $150 \mathrm{~km}$ to $500 \mathrm{~km}$ which trend towards the longer wavelengths at older ages. A shipboard study was also completed in this area and it observed $250 \mathrm{~m}$ topography anomalies correlated with the gravity and geoid anomalies. The short wavelength of these anomalies requires the existence of a low viscosity zone. In particular, the convective flow must be confined for some 
time to a low viscosity zone approximately $100-150 \mathrm{~km}$ in depth and with a viscosity contrast of one order of magnitude or more. At a Rayleigh number of $10^{5}-10^{7}$, a layer thickness of $125 \mathrm{~km}$ and a viscosity contrast of 0.01-0.001 taking into account the effect of the conducting lid thickness, the magnitude of the observed small scale anomalies, their persistence in magnitude until the development of longer wavelength convection and the departure of the depth-age relationship in the Pacific from a curve proportional to the square root of time can be reproduced.

A trend in the small wavelength convective anomalies to longer wavelengths with age is observed in the data and is also predicted by the model. In particular, as the small scale flow begins to decay into longer wavelength flows, the longer wavelength will dominate the surface observables. Because the transition from a $150 \mathrm{~km}$ wavelength to a $500 \mathrm{~km}$ wavelength is smooth, a Rayleigh number of over $10^{6}$ is indicated; and, since the small scale convective anomalies persist for $30-35 \mathrm{~m} \cdot \mathrm{y}$. in the geoid field, this long lifetime suggests a large viscosity contrast of over two orders of magnitude.

The onset of the convective flow is very young, at 5-10 m.y., and from the local critical Rayleigh number this onset time would indicate a viscosity contrast of at least 0.001 (Haxby and Weissel, 1986). However, since three dimensional coupling would cause the instability to occur much more 
quickly than indicated by these arguments, this large viscosity contrast may not be required by this onset time.

Finally, the predicted depth-age, geoid-age and heat flow-age relationships were compared to those observed in the oceans. We have shown that the small scale convective flow that is confined to the low viscosity zone does not greatly alter the depth-age relationship, although it produced a slightly elevated depths for a given age by 250 $1000 \mathrm{~m}$. In the region where the small scale anomalies are observed in the Pacific, the depths are slightly elevated by 250-750 $\mathrm{m}$ (McNutt and Fischer, 1987). The onset of the longer wavelength flow, however, radically affects the relationship, as predicted by Houseman and McKenzie (1982) and the depth-age curve flattens away from a halfspace cooling model. This prediction reconciles the observation of small scale convection at young ages in the Pacific with the departure of the depth-age relationship at $70 \mathrm{~m} . \mathrm{y} .$. Furthermore, this model predicts that the onset of longer wavelength flow will lead to the formation of longer wavelength features, such as swells, at ages greater than the age at which the depth-age curve departs from a halfspace model. It is observed that the onset of many swells in the Pacific and the Atlantic commences primarily at ages greater than $70 \mathrm{~m} . \mathrm{y}$. , such as the Hawaiian, Reunion; Society, Bermudan and Cape Verde swells (Crough, 1978). The onset of these mid-plate swells may result from this process 
and then can be viewed in part as a natural consequence of aging in the oceanic plates.

In conclusion, if the small scale anomalies that are observed in the southeast Pacific are due to a convection at depth, then a low viscosity zone exists which is $100-150 \mathrm{~km}$ thick with a viscosity contrast of more than 0.01 in an upper mantle which has a Rayleigh number of over $10^{6}$. The low viscosity zone confines the convective flow to shallow depths for a period, but eventually convective flow is instigated throughout the upper mantle. The small scale convective flow has only small effects on the depth-age relationship until the onset of this mantle-wide flow, at which point the depth-age curve flattens as predicted by the plate model (Houseman and McKenzie, 1982). The heat flow age curve flattens earlier than the plate model, but the predicted heat flow values agree well with heat flow measurements on old Atlantic seafloor (Louden et al., 1987). The onset of the longer wavelength flow also produces features which resemble mid-plate swells after the onset of long wavelength convection (i.e. after the flattening of the depth-age curves).

\section{Acknowledgments}

This work has been supported by NASA Geodynamics program grant NAG5-415, and National Science Foundation grant EAR-8306249. 
Table 5.1

Variable Description

\section{$\rho_{0}$}

$\rho_{W}$

90

K

$\alpha$

$\mathrm{T}_{\mathrm{e}}$

$v$

$\Delta \mathrm{T}$

E

$\mathrm{d}^{2} / \mathrm{k}$ depth scale

average mantle density

density of water

surface gravitational acceleration

average mantle thermal diffusivity

average mantle thermal expansion coefficient

elastic plate thickness

Poisson's ratio

Temperature contrast across the box

Young's Modulus

scaling time
Value

$600 \mathrm{~km}$

$3330 \mathrm{~kg} / \mathrm{m}^{3}$

$1025 \mathrm{~kg} / \mathrm{m}^{3}$

$9.82 \mathrm{~m} / \mathrm{s}^{2}$

$10^{-6} \mathrm{~m}^{2} / \mathrm{s}$

$3.1 \times 10^{-5}{ }^{\circ} \mathrm{C}^{-1}$

$10 \mathrm{~km}$

0.25

$1365^{\circ} \mathrm{C}$

$8 \times 10^{10} \mathrm{~N} / \mathrm{m}^{2}$

$11407 \mathrm{~m} \cdot \mathrm{y}$. 


\section{Table 5.2}

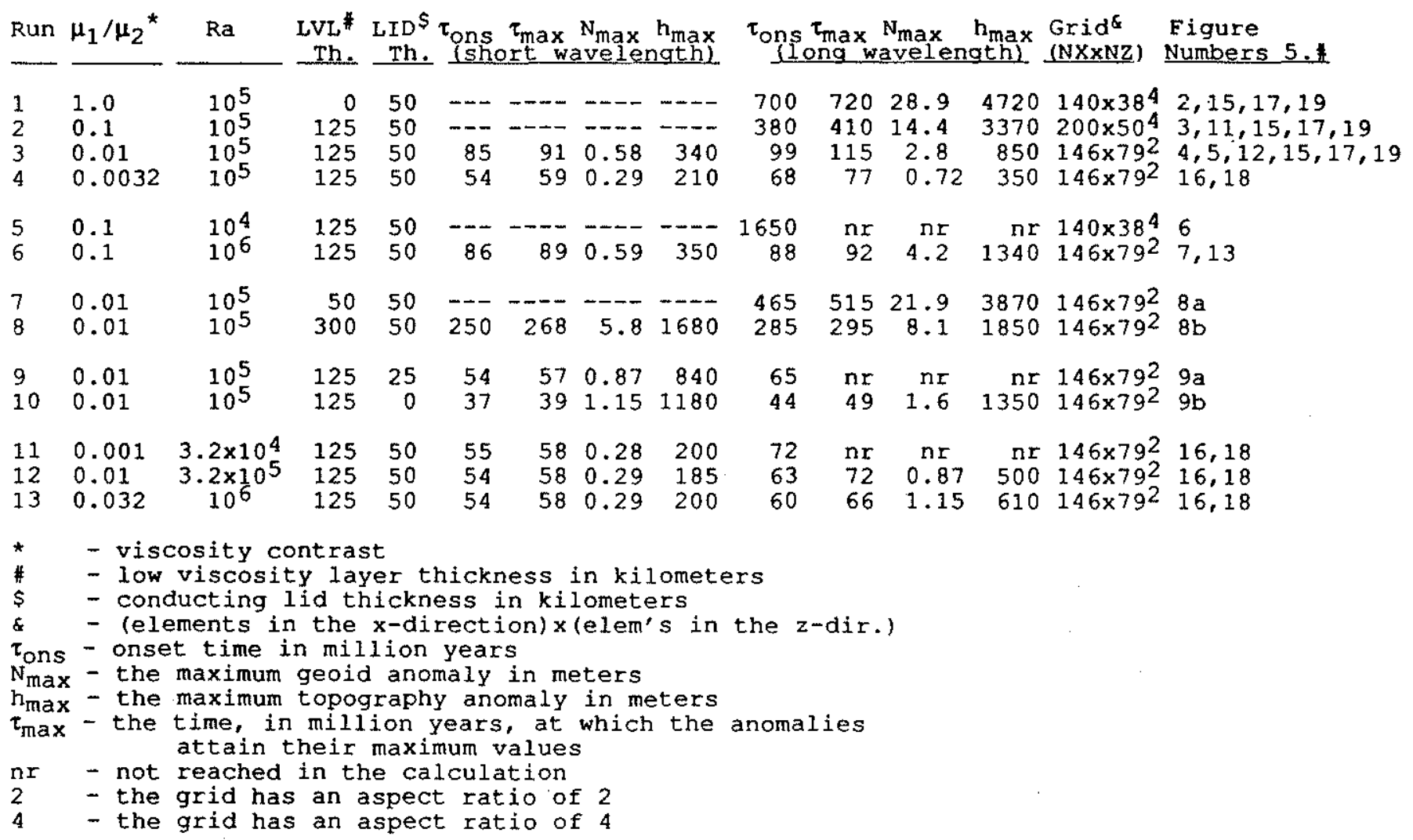




\section{Figure Captions}

Fiqure 5.1: Geometry and boundary conditions of the model where "a" is the thickness of the bottom layer, "b" is the thickness of the whole box, and "h" is the width of the box.

Figure 5.2: Temperature contours for run 1 in Table 5.2, at $720 \mathrm{~m} . \mathrm{y}$. and $770 \mathrm{~m} . \mathrm{y}$. , where the Rayleigh number is $10^{5}$, the low viscosity layer thickness is $125 \mathrm{~km}$, the lid thickness is $50 \mathrm{~km}$ and there is no viscosity contrast. The ticks mark the bottom of the conducting lid. The temperature contours mark $136^{\circ} \mathrm{C}$ intervals.

Figure 5.3: Temperature contours for run 2 in Table 5.2, at $400 \mathrm{~m} . \mathrm{y}$. and $450 \mathrm{~m} . \mathrm{y} .$, where the Rayleigh number is $10^{5}$, the low viscosity layer thickness is $125 \mathrm{~km}$, the lid thickness is $50 \mathrm{~km}$ and the viscosity contrast is 0.1 . The ticks mark the boundaries of the low viscosity zone. The temperature contours mark $136^{\circ} \mathrm{C}$ intervals.

Figure 5.4: Temperature contours for run 3 in Table 5.2, at $91 \mathrm{~m} \cdot \mathrm{y} \cdot 110 \mathrm{~m} \cdot \mathrm{y}$. and $130 \mathrm{~m} \cdot \mathrm{y}$. , where the Rayleigh number is $10^{5}$, the low viscosity layer thickness is $125 \mathrm{~km}$, the lid thickness is $50 \mathrm{~km}$ and the viscosity contrast is 0.01 . The ticks mark the boundaries of the low viscosity zone. The temperature contours mark $136^{\circ} \mathrm{C}$ intervals. 
Figure 5.5: (a) The nondimensional kinetic energy versus dimensional time for run 3 in Table 5.2, where the Rayleigh number is $10^{5}$, the low viscosity layer thickness is $125 \mathrm{~km}$, the lid thickness is $50 \mathrm{~km}$ and the viscosity contrast is 0.01 . The first peak corresponds to the onset and development of the small scale convection with the onset time marked by $\tau_{\mathrm{S}}$. The second peak corresponds to the onset and development of convection of a longer wavelength with the onset time marked by $\tau_{1}$. (b) The horizontally averaged nondimensional temperature with the nondimensional depth for run 3 in Table 5.2. The solid line is at $60 \mathrm{~m} . \mathrm{y}$.; the long dashed line is at $91 \mathrm{m.y.;}$ the medium dashed line is at 110 $\mathrm{m} . \mathrm{y}$. and the short dashed line is at $130 \mathrm{~m} . \mathrm{y} .$.

Figure 5.6: Temperature contours for run 5 in Table 5.2, where the Rayleigh number is $10^{4}$, the low viscosity layer thickness is $125 \mathrm{~km}$, the conducting lid thickness is $50 \mathrm{~km}$ and the viscosity contrast is 0.1. The ticks mark the boundary of the low viscosity zone and the temperature contours represent intervals of $136^{\circ} \mathrm{C}$.

Figure 5.7: Temperature contours for run 6 in Table 5.2, where the Rayleigh number is $10^{6}$, the low viscosity layer thickness is $125 \mathrm{~km}$, the conducting lid thickness is $50 \mathrm{~km}$ and the viscosity contrast is 0.1 . The ticks mark the 
boundary of the low viscosity zone and the temperature contours represent intervals of $136^{\circ} \mathrm{C}$.

Figure 5.8: Temperature contours for (a) run 7 and (b) run 8 in Table 5.2, where the Rayleigh number is $10^{5}$, the low viscosity layer thickness is (a) $50 \mathrm{~km}$ and (b) $300 \mathrm{~km}$, the conducting lid thickness is $50 \mathrm{~km}$, and the viscosity contrast is 0.1 . The ticks mark the boundary of the low viscosity zone and the temperature contours represent intervals of $136^{\circ} \mathrm{C}$.

Eigure 5.9: Temperature contours for (a) run 9 and (b) run 10 in Table 5.2, where the Rayleigh number is $10^{5}$, the low viscosity layer thickness is $125 \mathrm{~km}$, the conducting lid thickness is (a) $25 \mathrm{~km}$ and (b) $0 \mathrm{~km}$, and the viscosity contrast is 0.01 . The ticks mark the boundary of the low viscosity zone and the temperature contours represent intervals of $136^{\circ} \mathrm{C}$.

Figure 5.10: The convective onset time, as defined in the text, versus the viscosity contrast, for runs where the low viscosity layer thickness is $125 \mathrm{~km}$ and the conducting lid thickness is $50 \mathrm{~km}$. We have plotted the runs in three groups with different Rayleigh numbers $\left(10^{4}, 10^{5}\right.$ and $\left.10^{6}\right)$. Some of the results presented here are from runs not listed in Table 5.2. 
Figure 5.11: The geoid, topography, gravity and heat flow anomalies across the top of the box at $400 \mathrm{~m} \cdot \mathrm{y}$. for run 2 in Table 5.2. The Rayleigh number is $10^{5}$, the low viscosity layer thickness is $125 \mathrm{~km}$, the conducting lid thickness is $50 \mathrm{~km}$ and the viscosity contrast is 0.1 .

Figure 5.12: The geoid, topography, gravity and heat flow anomalies across the top of the box for run 3 in Table 5.2. The Rayleigh number is $10^{5}$, the low viscosity layer thickness is $125 \mathrm{~km}$, the conducting lid thickness is $50 \mathrm{~km}$ and the viscosity contrast is 0.01 . (a) at $91 \mathrm{~m} . \mathrm{y} . ;$ and (b) at $110 \mathrm{~m} \cdot \mathrm{y} . \mathrm{x}$

Figure 5.13: The geoid, topography, gravity and heat flow anomalies across the top of the box at $89 \mathrm{~m} . \mathrm{y}$. for run 6 in Table 5.2. The Rayleigh number is $10^{6}$, the low viscosity layer thickness is $125 \mathrm{~km}$, the conducting lid thickness is $50 \mathrm{~km}$ and the viscosity contrast is 0.1 .

Figure 5.14: The topography and gravity kernels at a wavelength of eight times the depth of the fluid layers. The low viscosity layer thickness is 0.21 and it lies between 0.79 and 1.0 in the depth scale. The solid lines are the kernels with no viscosity contrast; the long dash Iines represent a viscosity contrast of 0.1 ; the medium dash lines represent a viscosity contrast of 0.01 ; and the short dash lines represent a viscosity contrast of 0.001 . 
Figure 5.15: The subsidence-age relationships (versus the square root of the age) for runs 1 (solid line), 2 (long dash), 3 (medium dash) and 4 (short dash). See Table 5.2 for details of the runs. The geometry of the model and the Rayleigh number are the same in each of these runs, but we vary the viscosity contrast from 1.0 to 0.0032 (noted by each curve).

Figure 5.16: The subsidence-age relationships (versus the square root of the age) for runs 13 (solid line), 12 (long dash), 4 (medium dash) and 11 (short dash). See Table 5.2 for details of the runs. The geometry of the viscosity structure and the Rayleigh number based on the viscosity of the top layer is $3.2 \times 10^{7}$ in each of these calculations, but we vary the Rayleigh number based on the viscosity in the bottom layer from $3.2 \times 10^{4}$ to $10^{6}$.

Figure 5.17: The geoid-age relationships for runs 1 (solid line), 2 (long dash), 3 (medium dash) and 4 (short dash), as in Figure 5.15. The viscosity contrast is noted by each curve.

Figure 5.18: The geoid-age relationships for runs 13 (solid line), 12 (long dash), 4 (medium dash) and 11 (short dash), as in Figure 5.16. The viscosity contrast is noted by each curve. 
Figure 5.19: The heat flow-age relationships (versus the square root of the age) for runs 1 (solid line), 2 (long dash), 3 (medium dash) and 4 (short dash), as in Figure 5.15. The viscosity contrast is noted by each curve. 


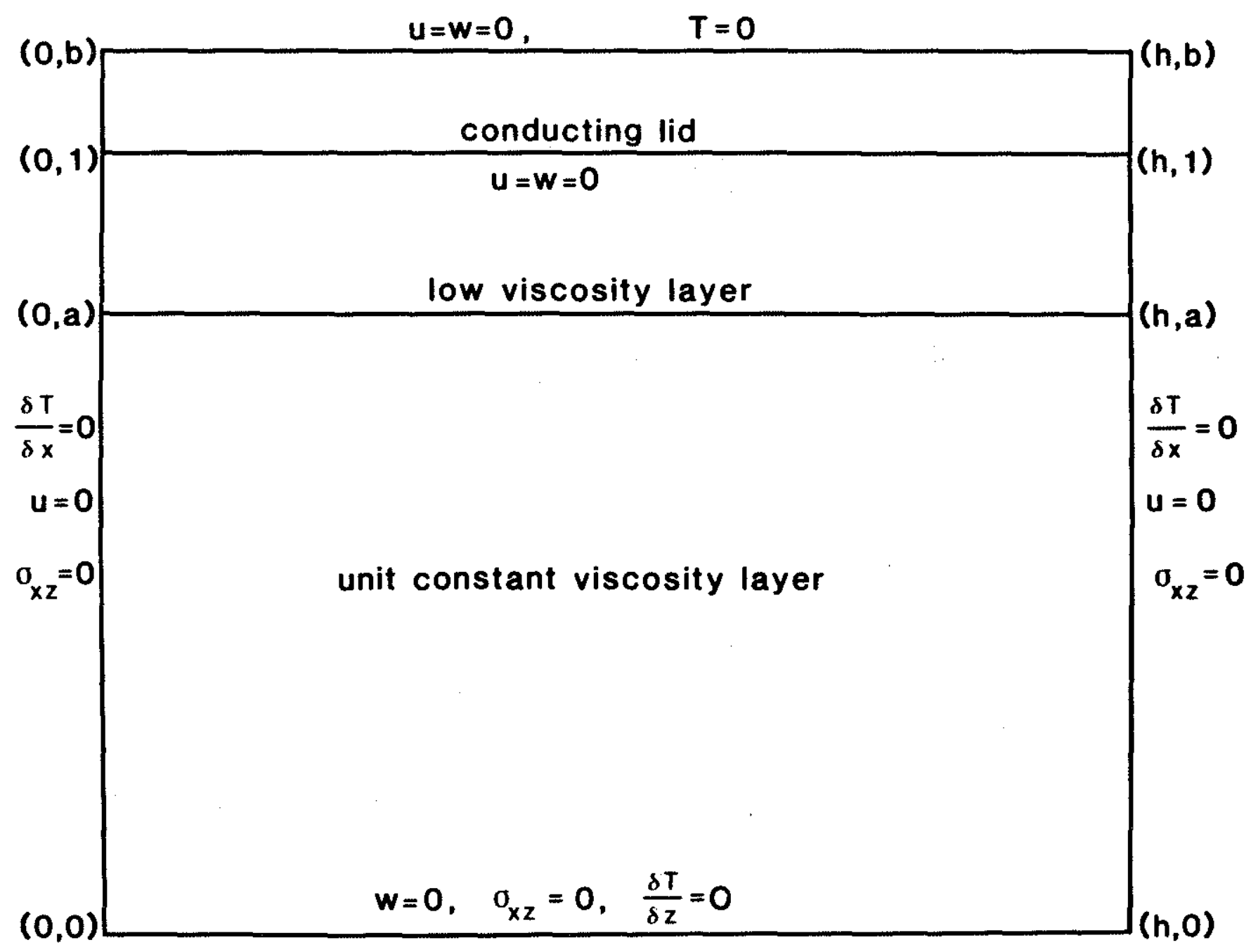

Figure 5.1 

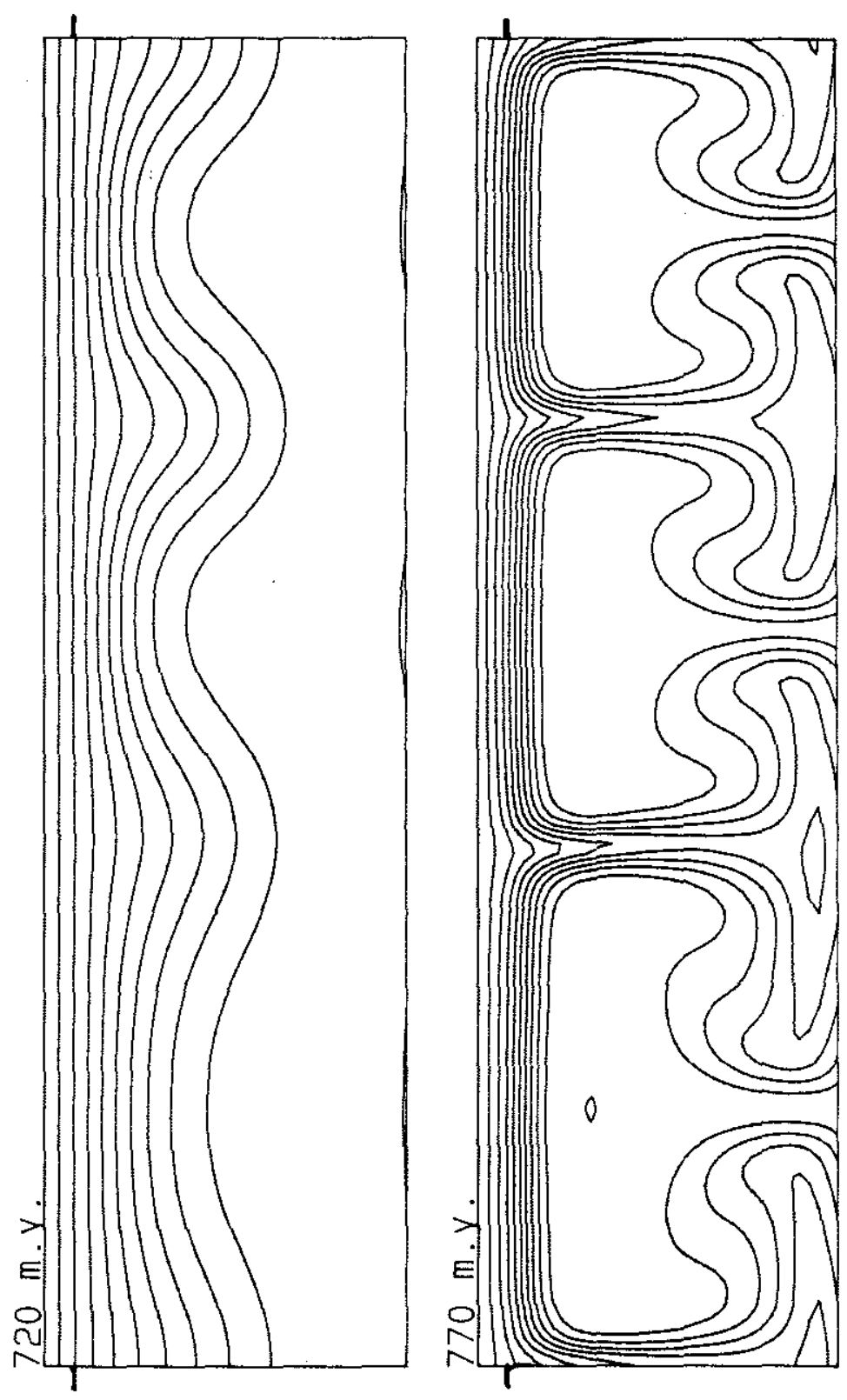

ำ 

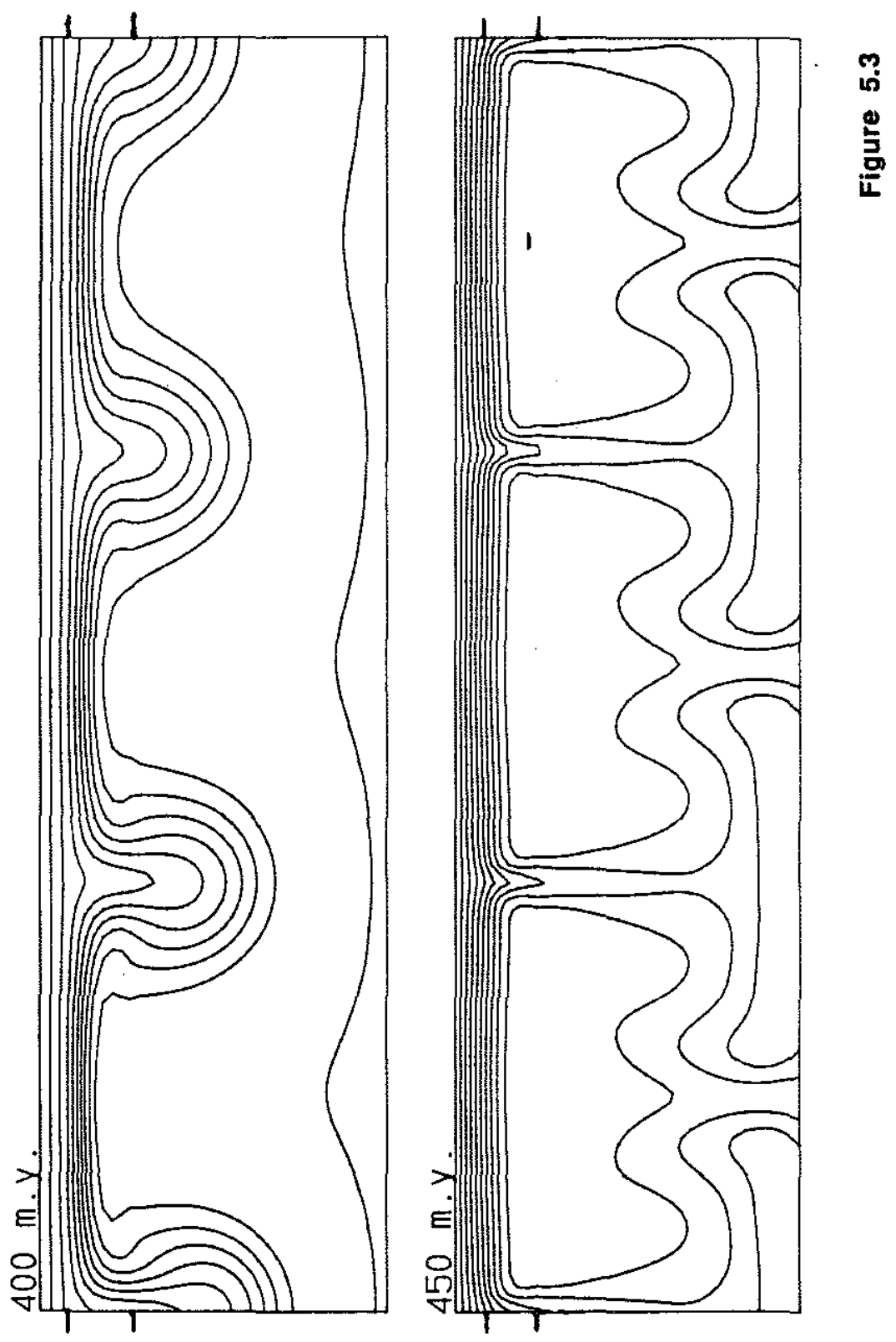

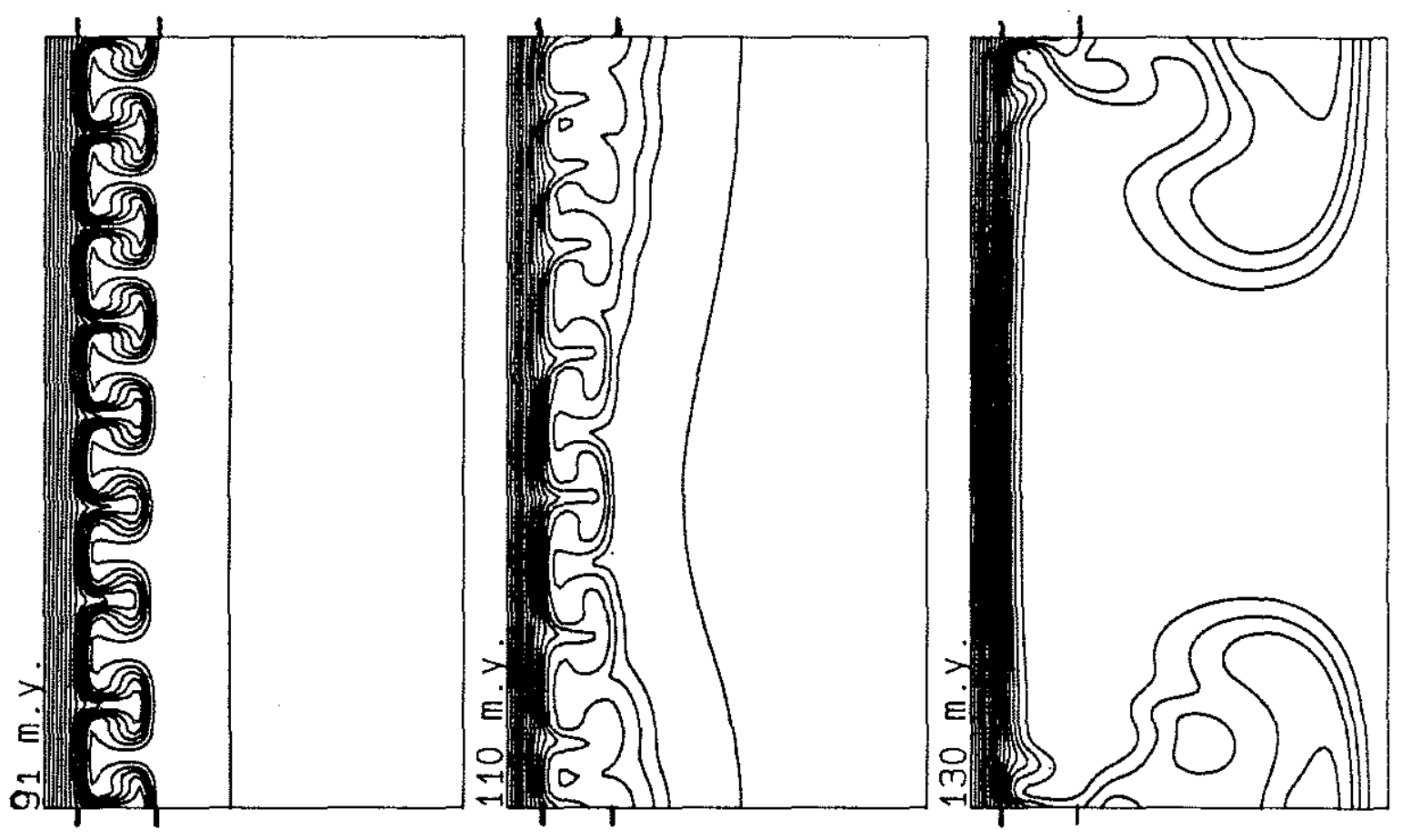


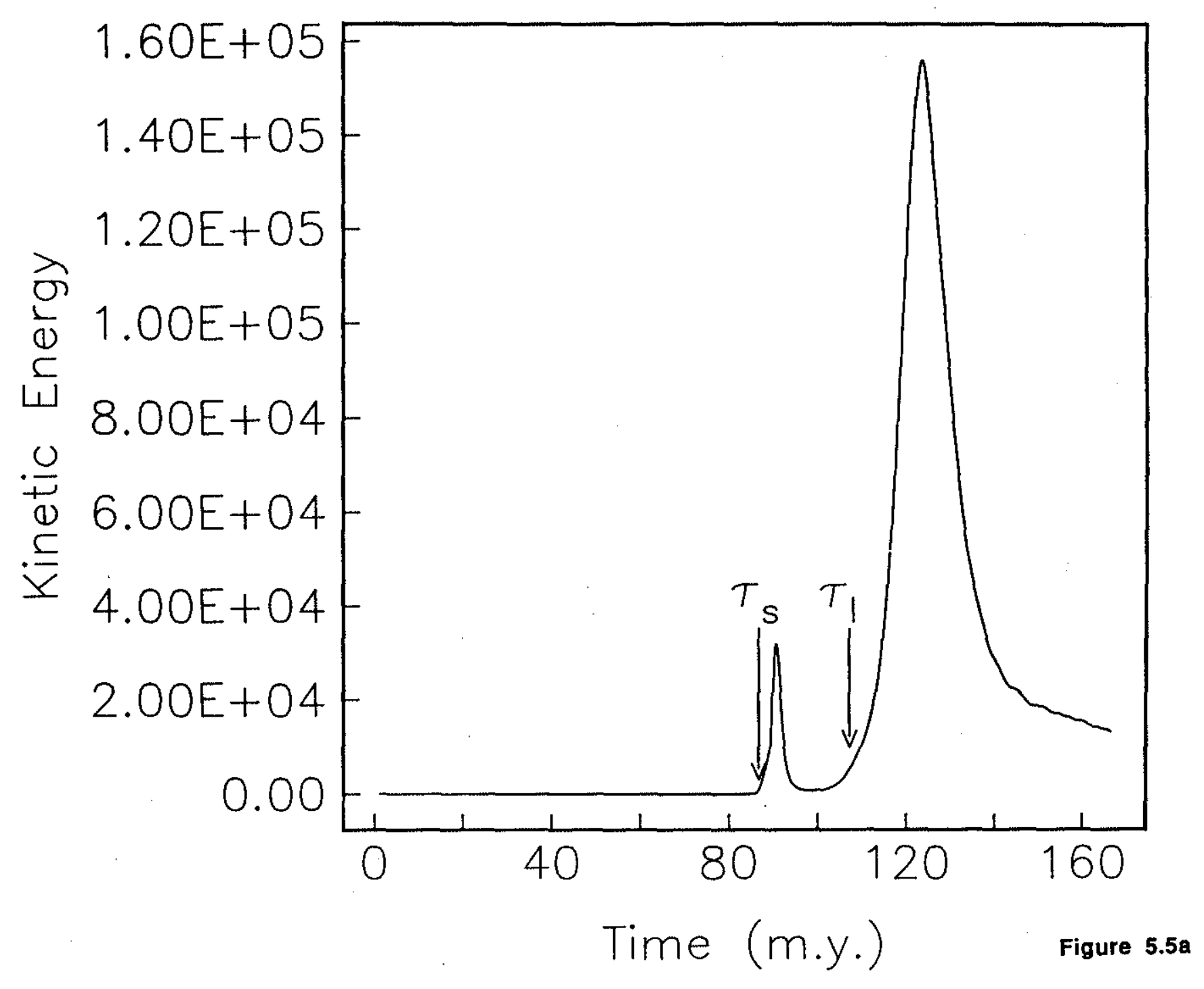




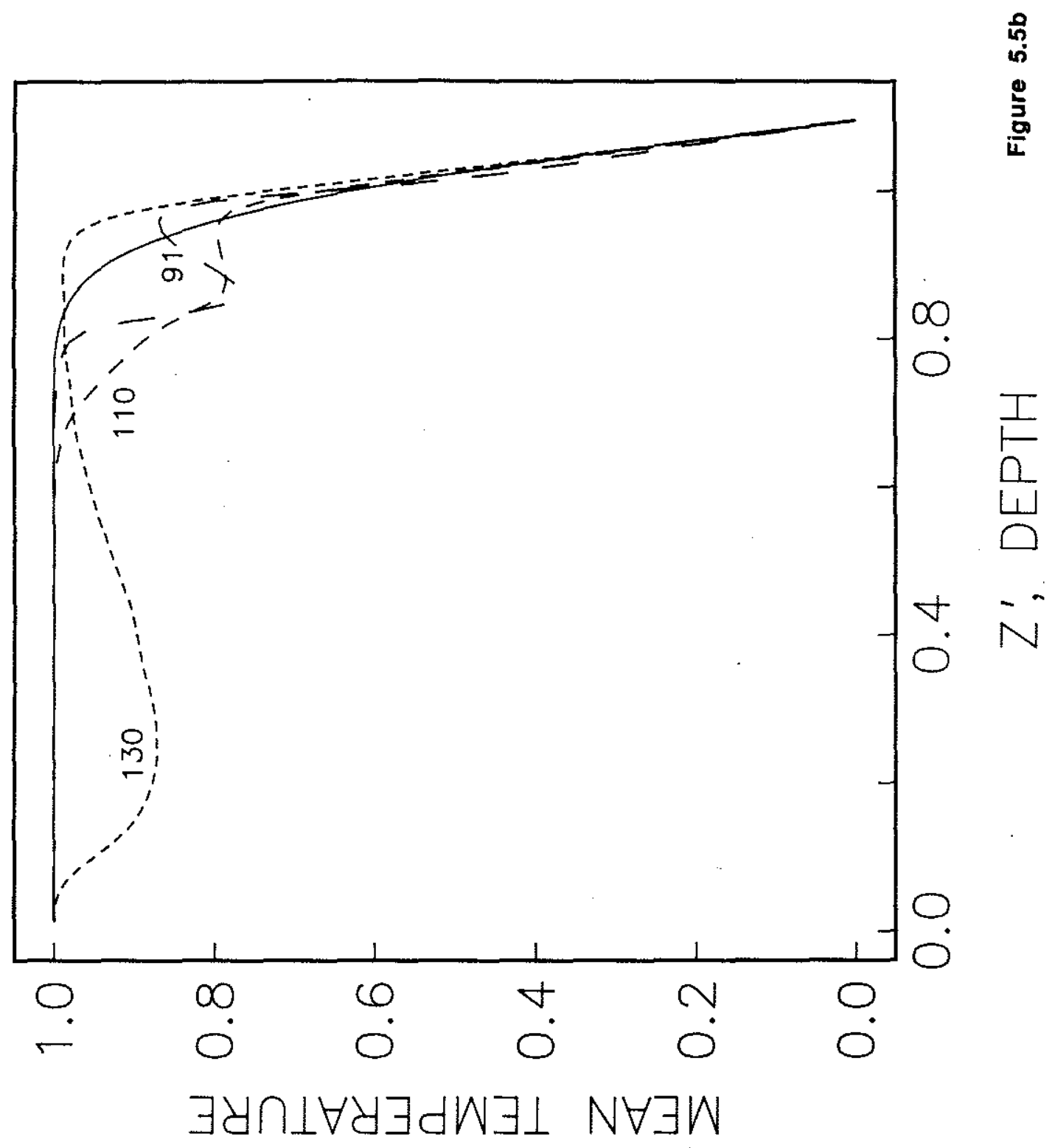




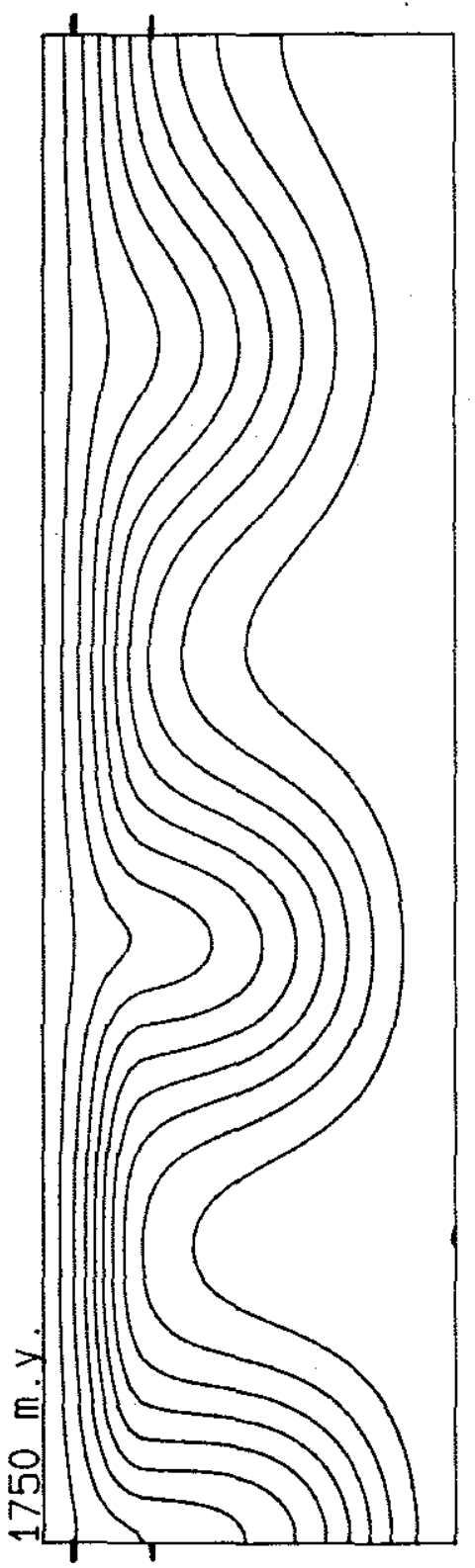

แั 


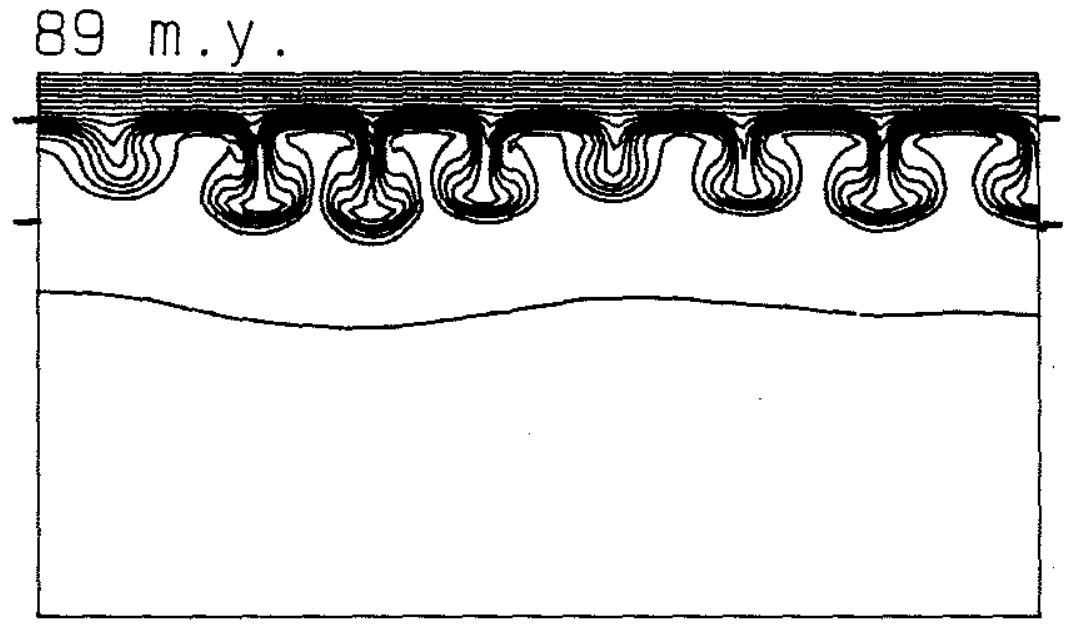

Figure 5.7 


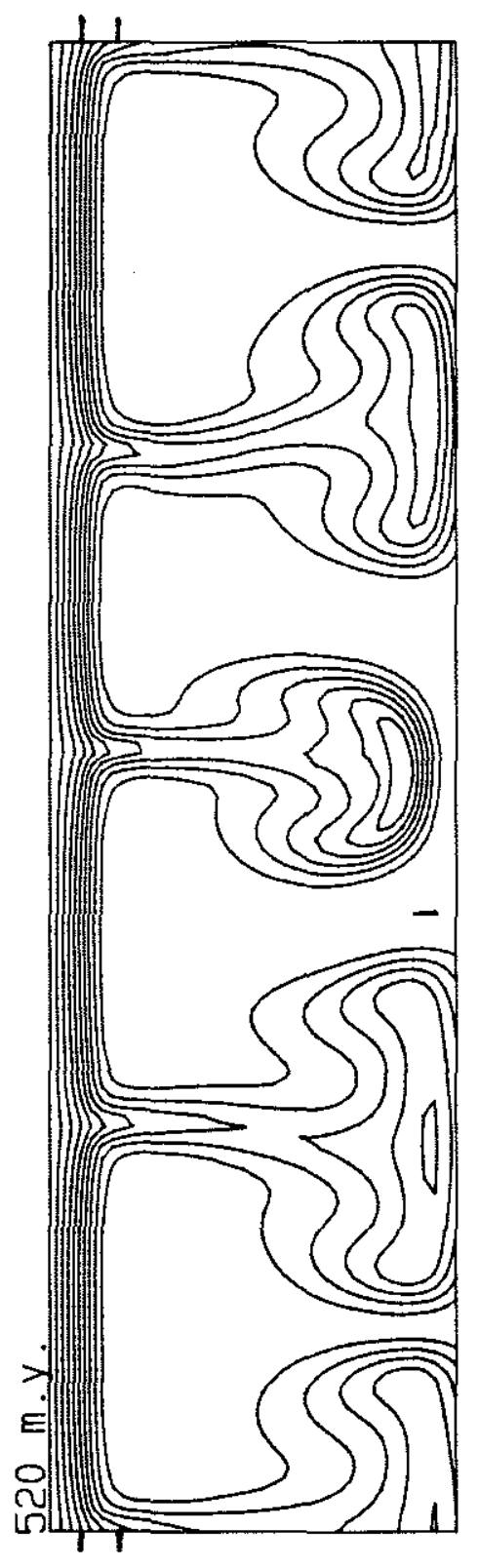

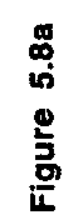




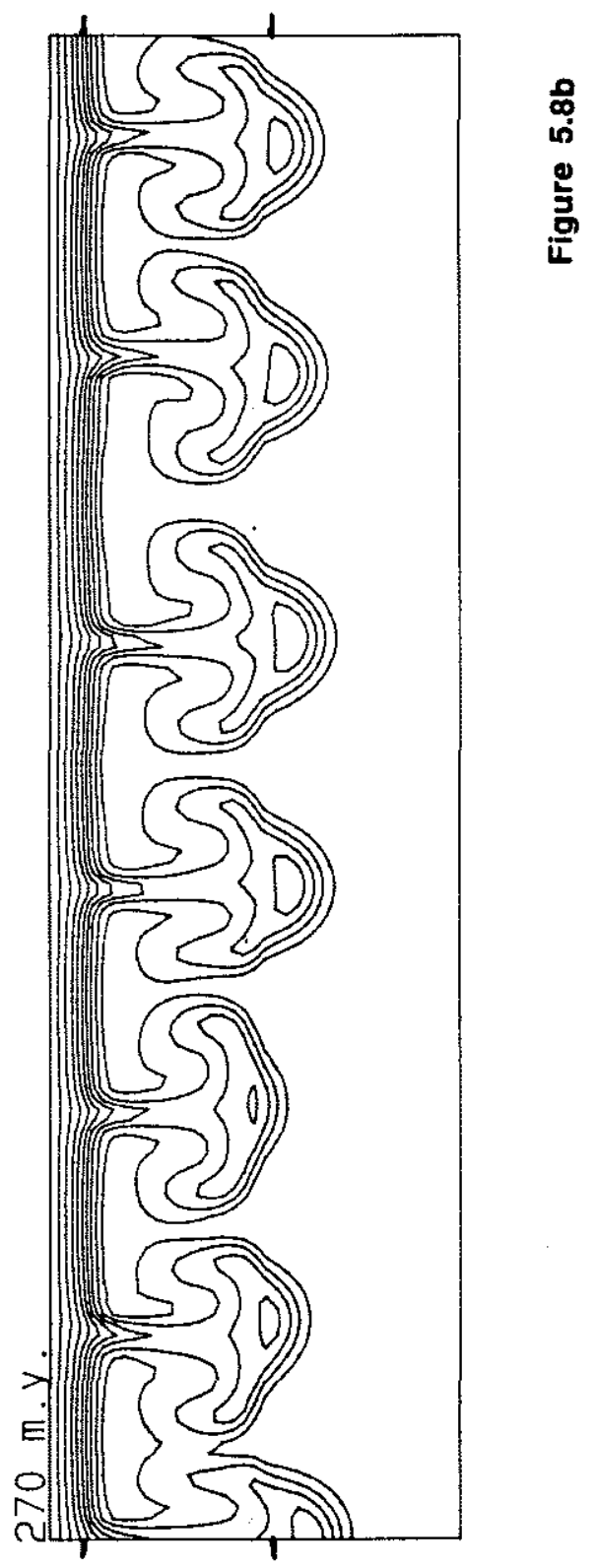




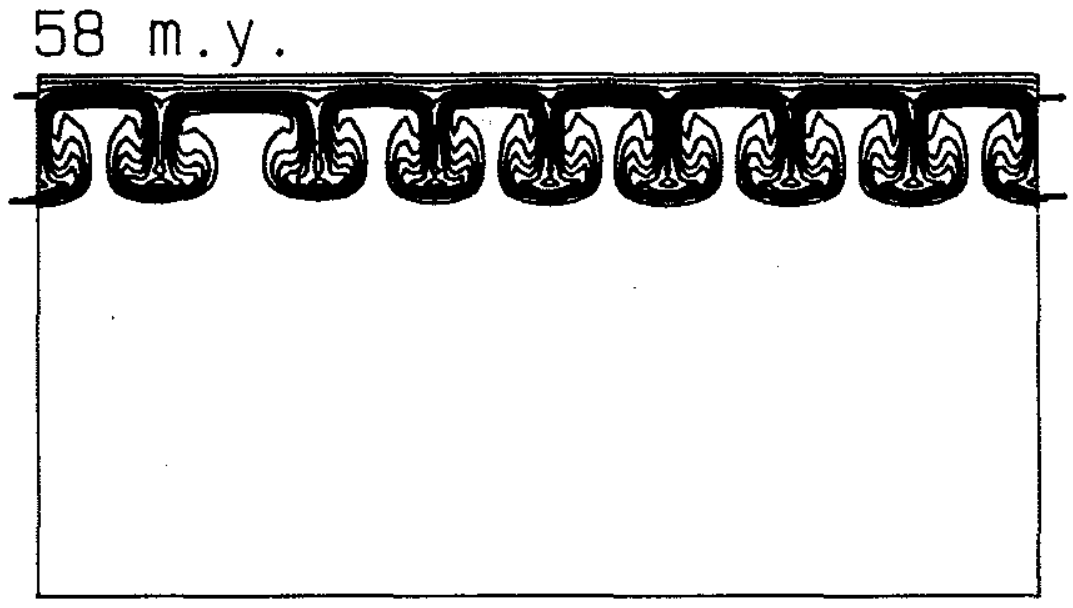

Figure 5.9a 


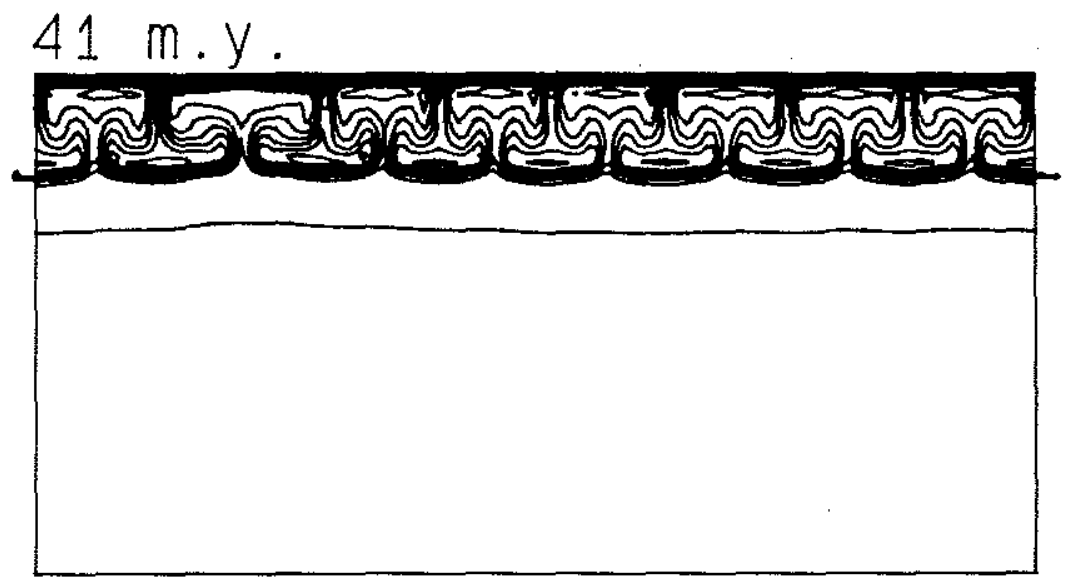

Figure $5.9 \mathrm{~b}$ 
0
10
욱
인

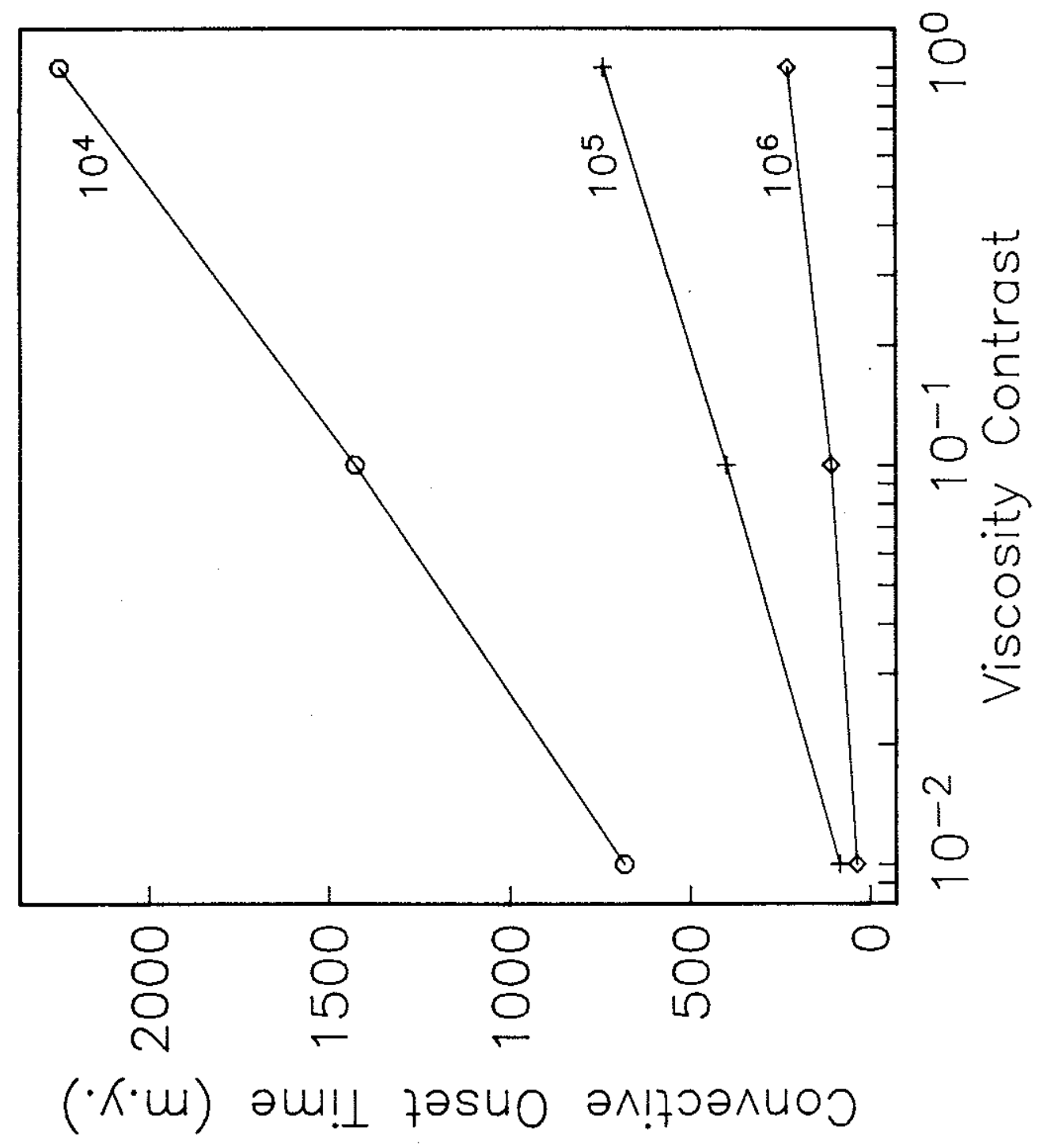



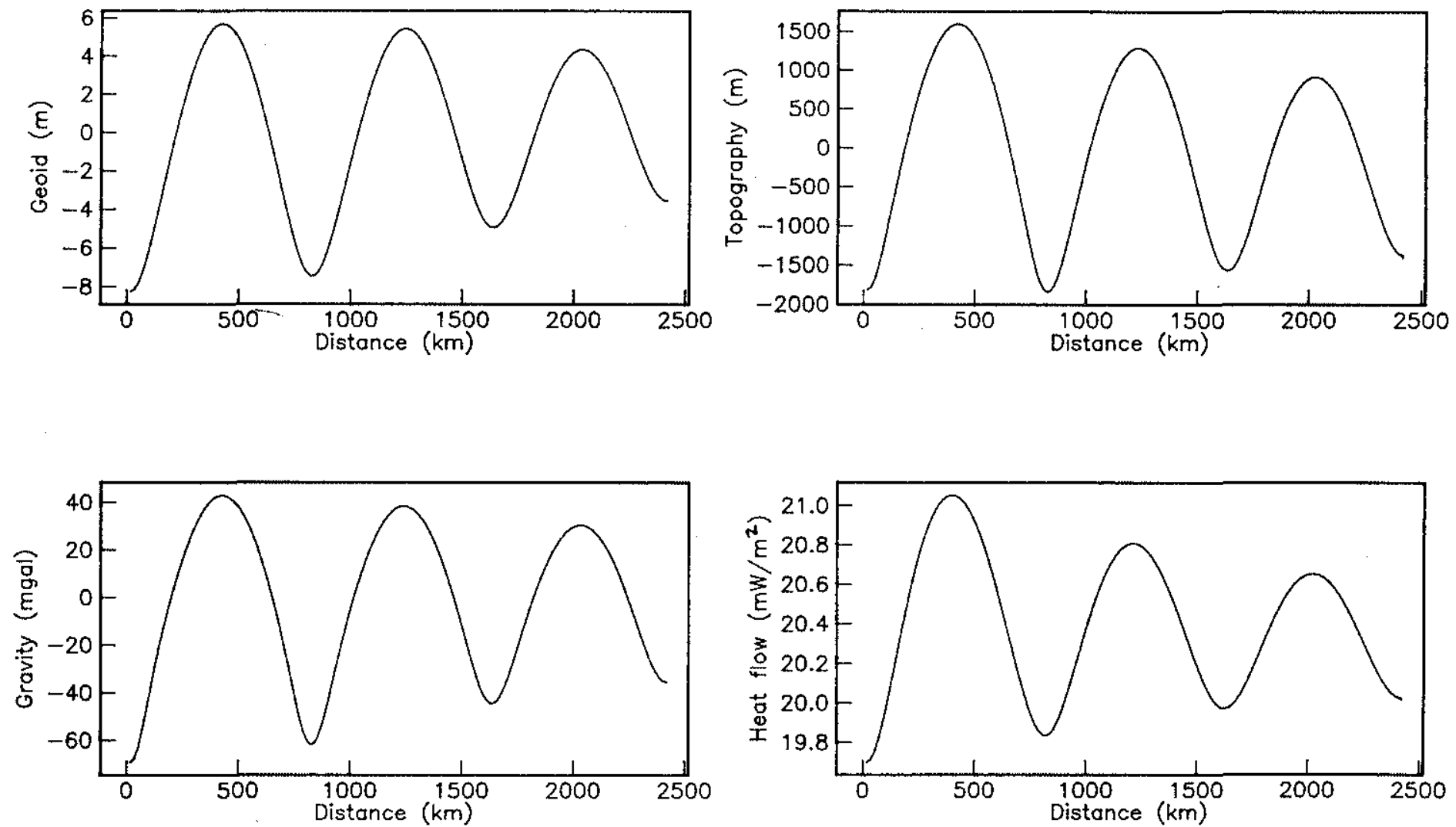

Figure 5.11 

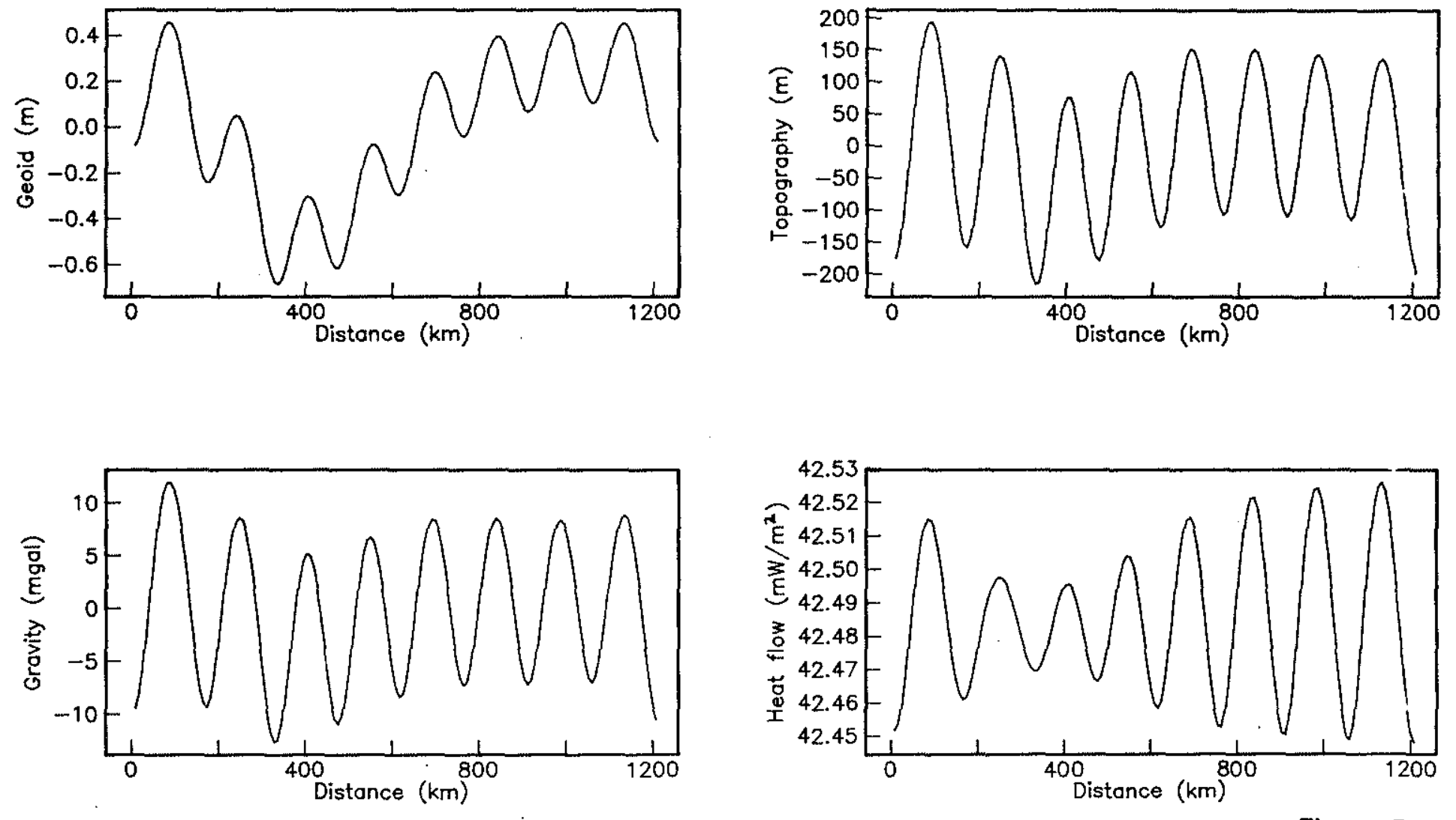

Figure $5.12 a$ 

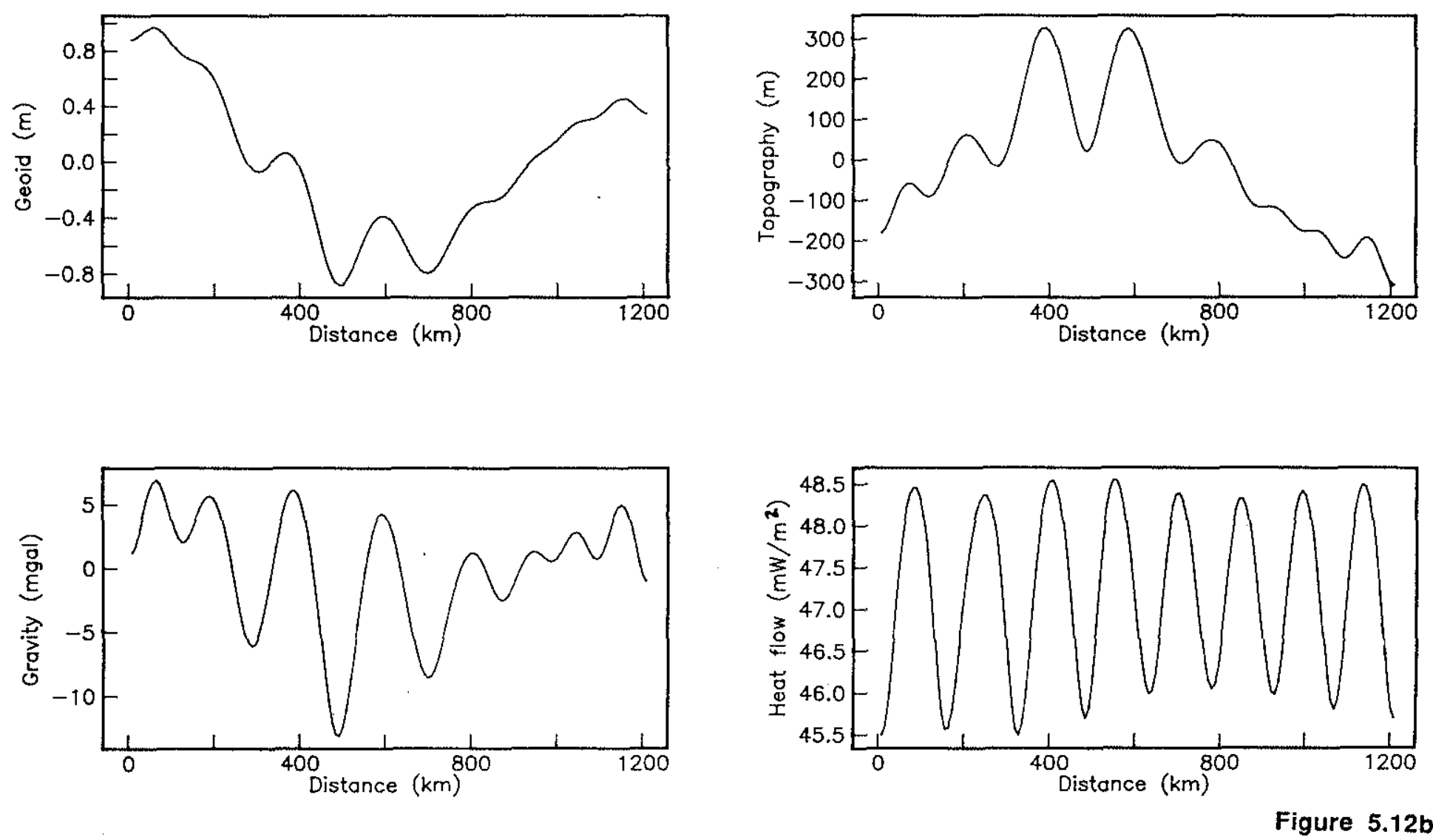

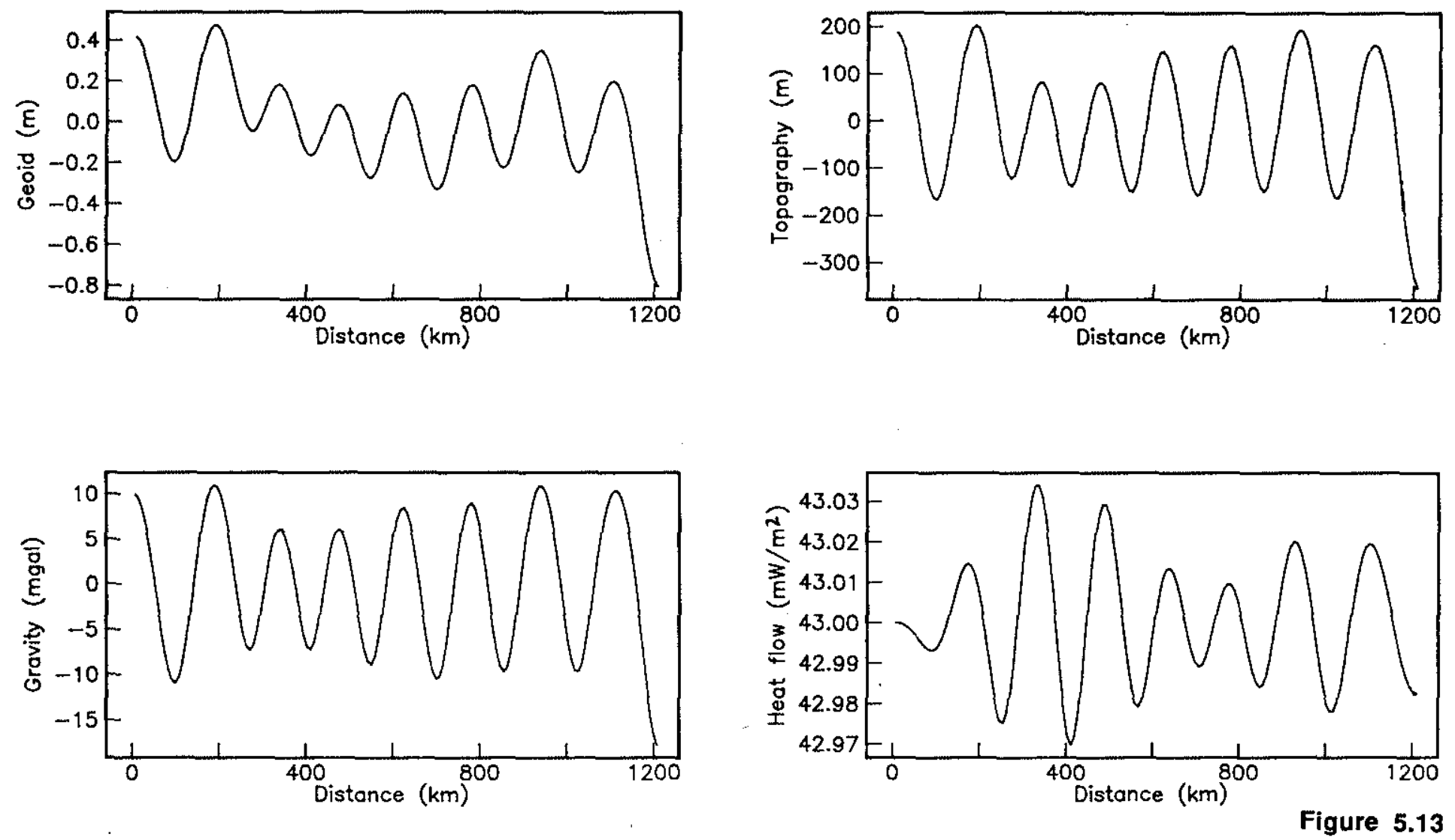


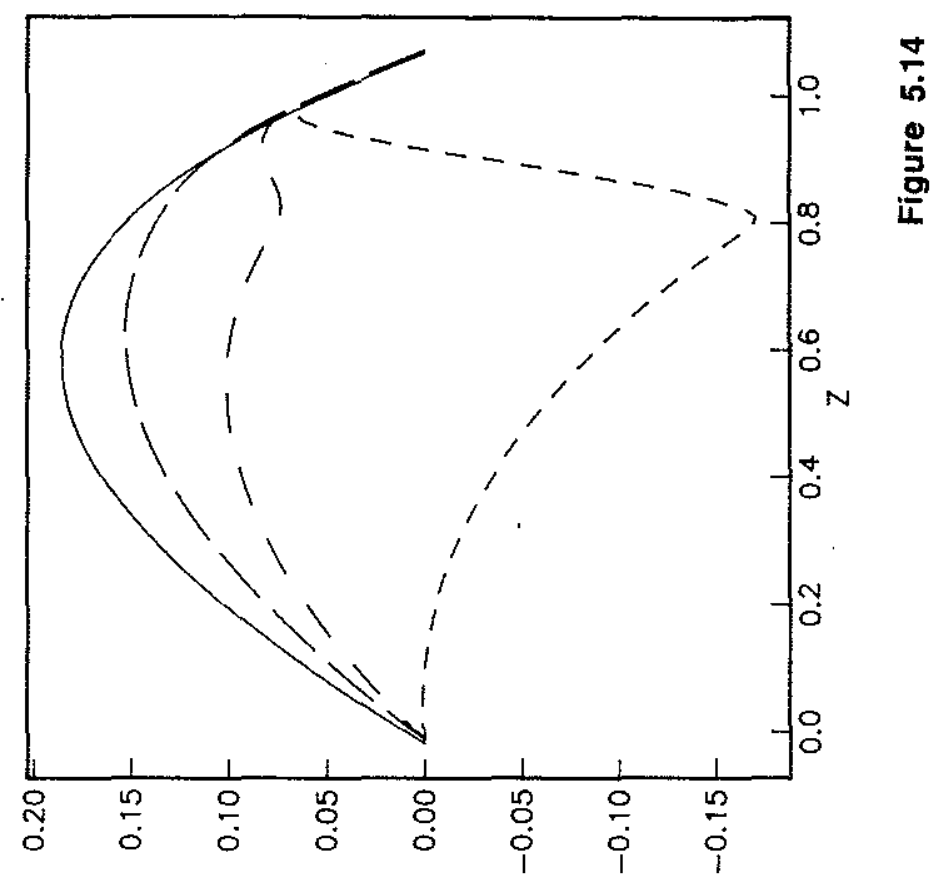

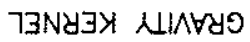

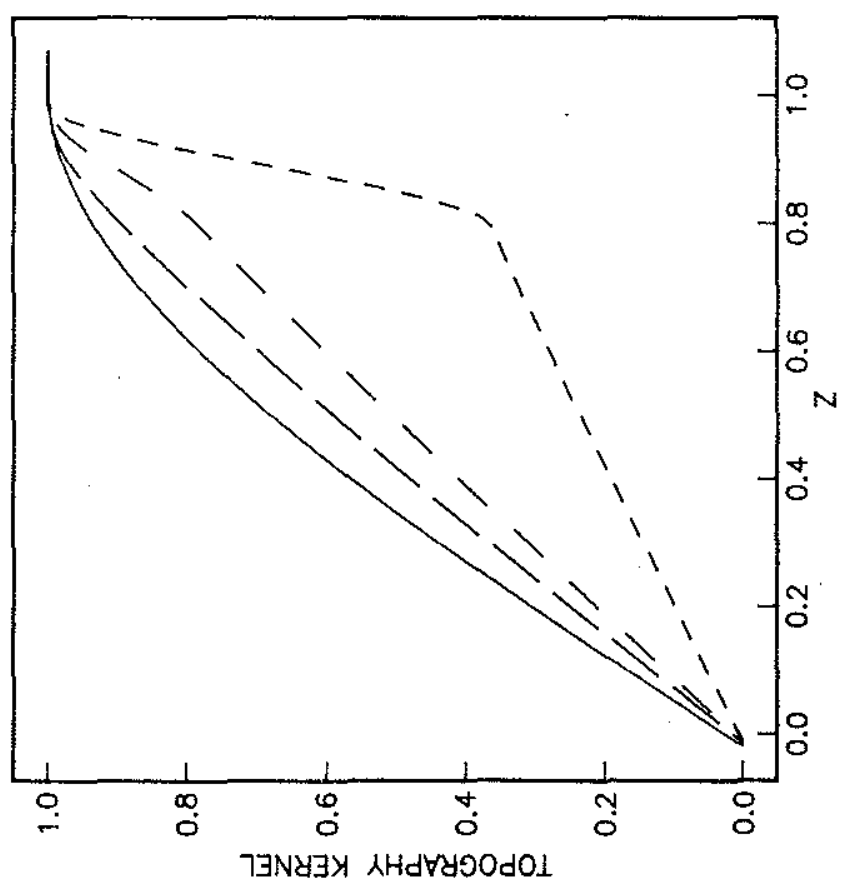




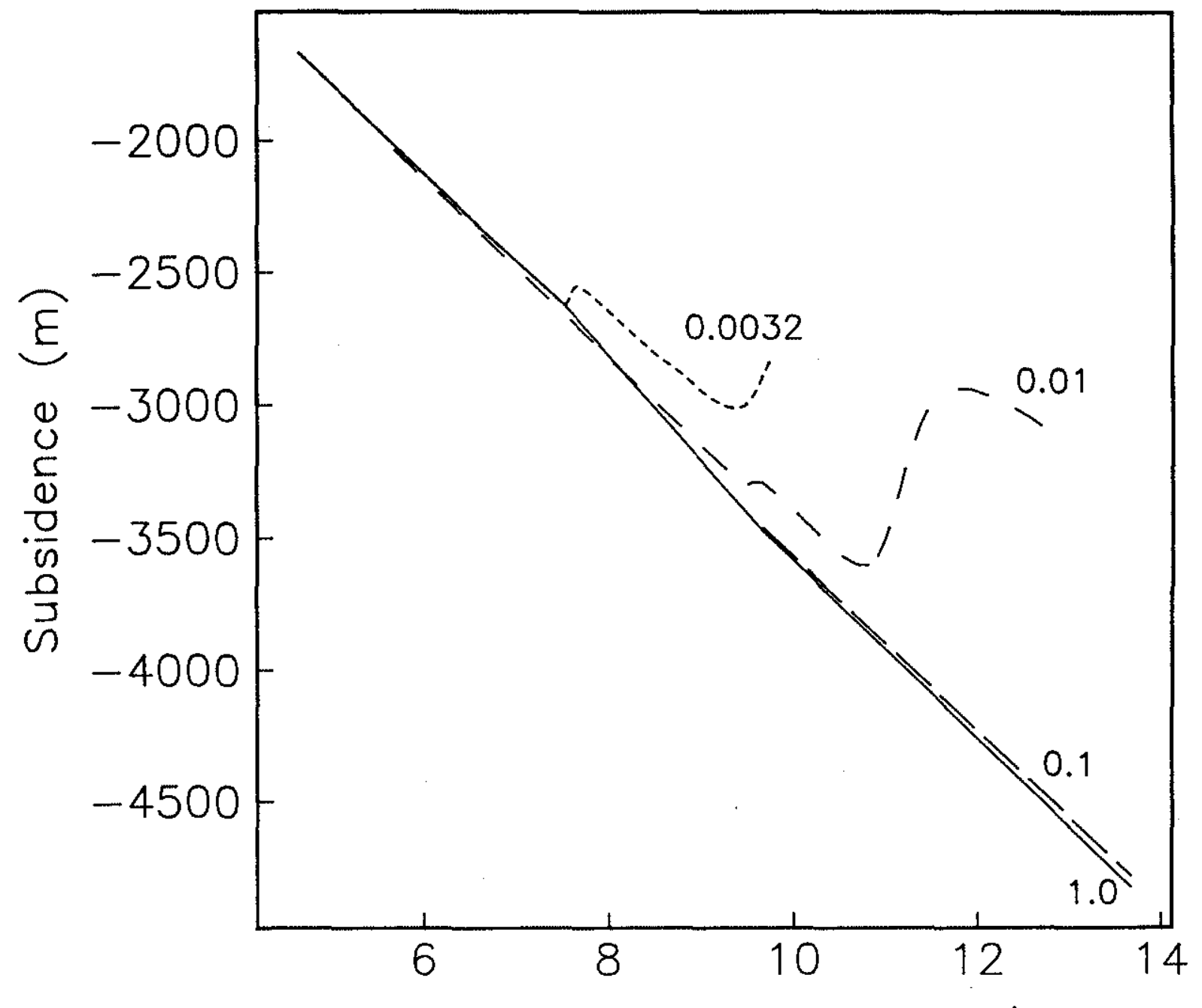

Squareroot of Time (m.y. ${ }^{1 / 2}$ ) Figure 5.15 


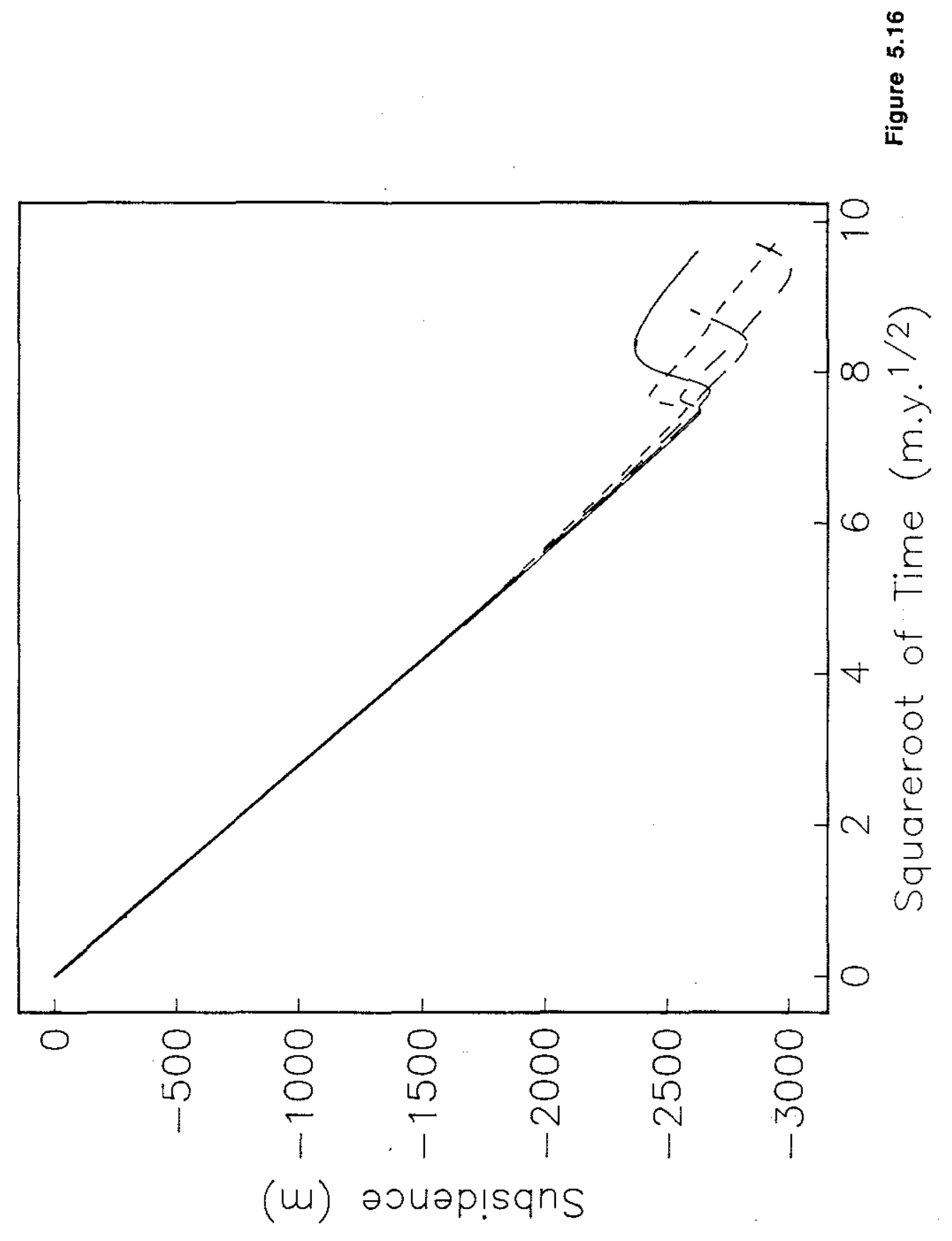




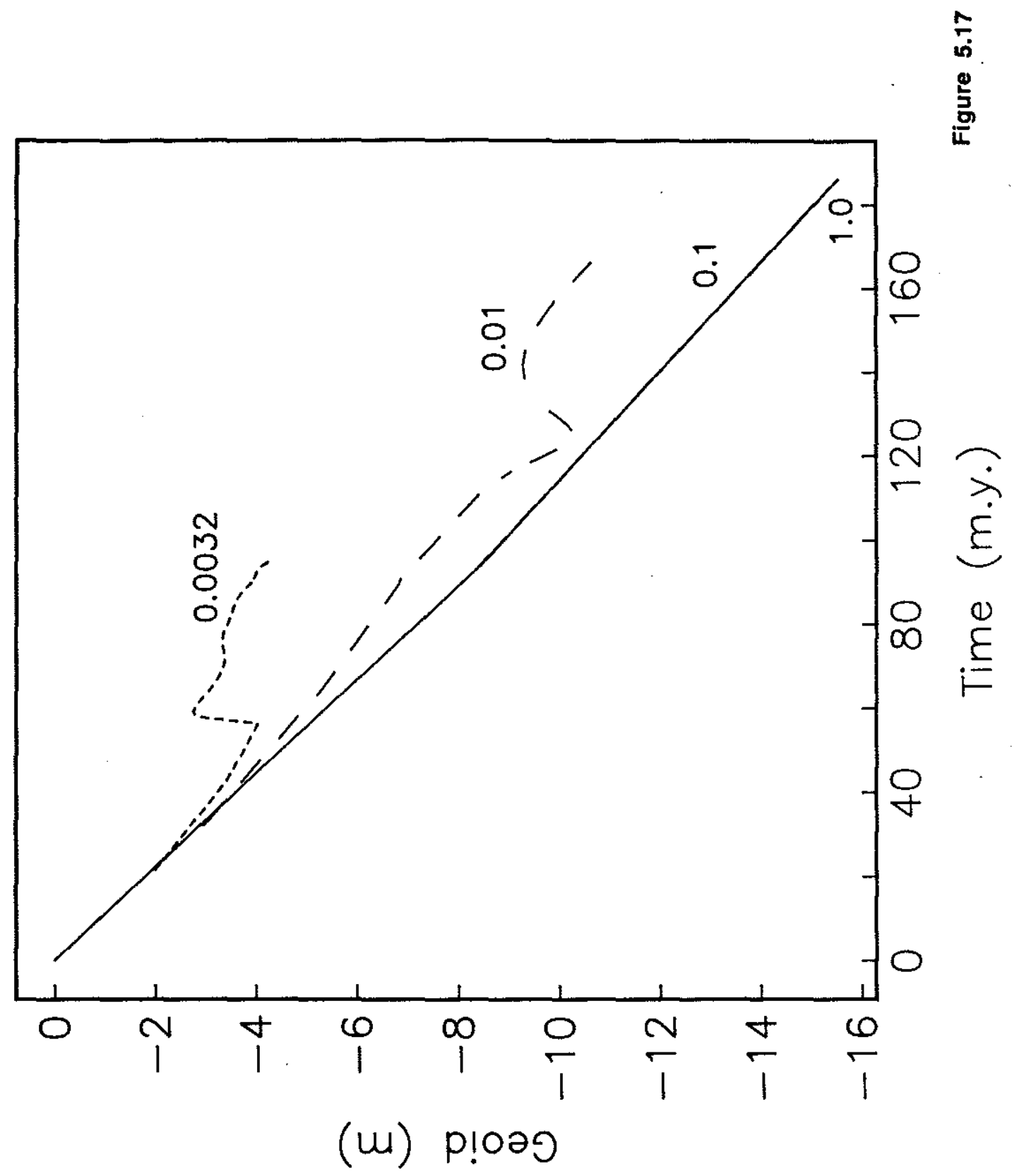




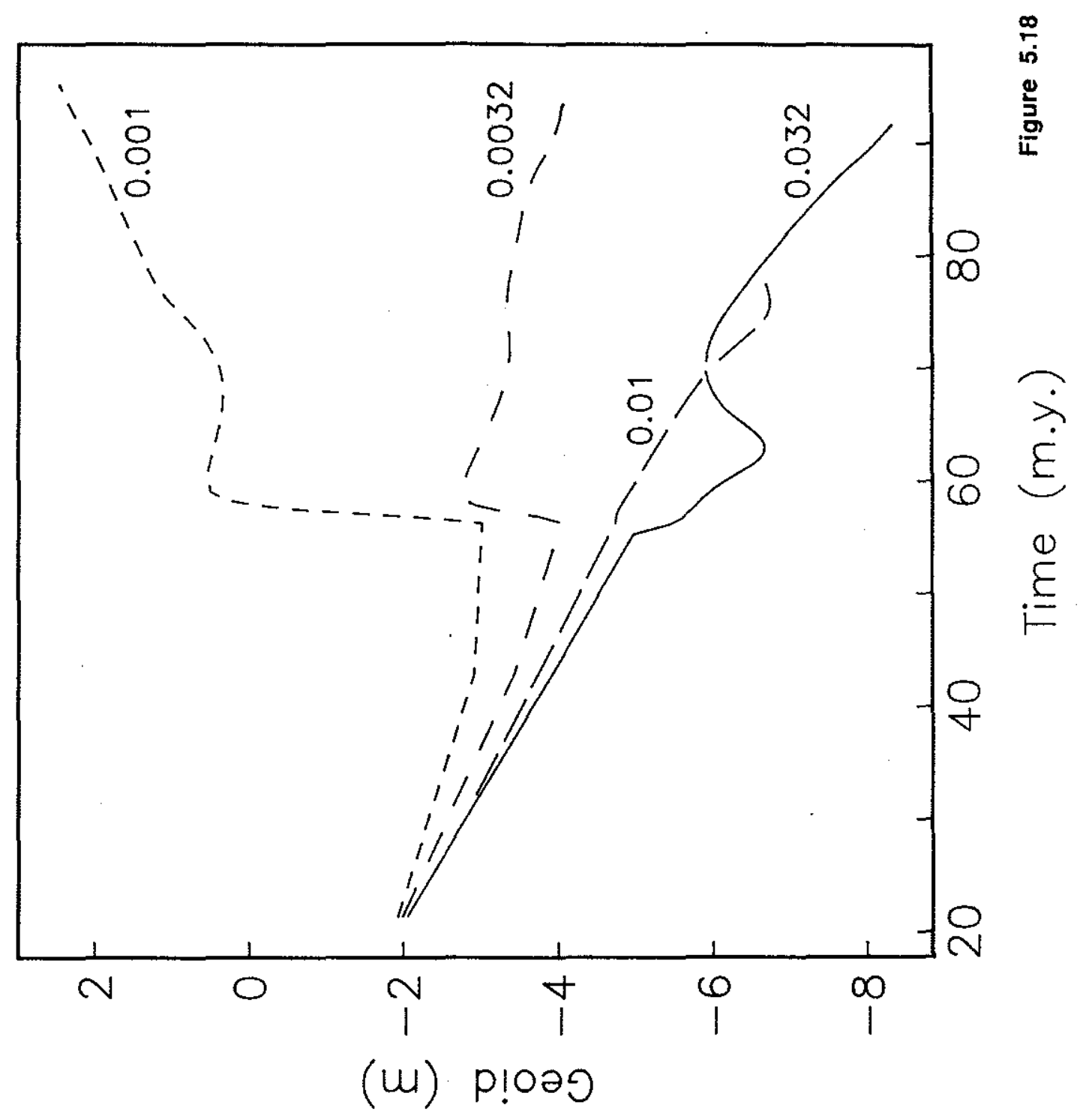




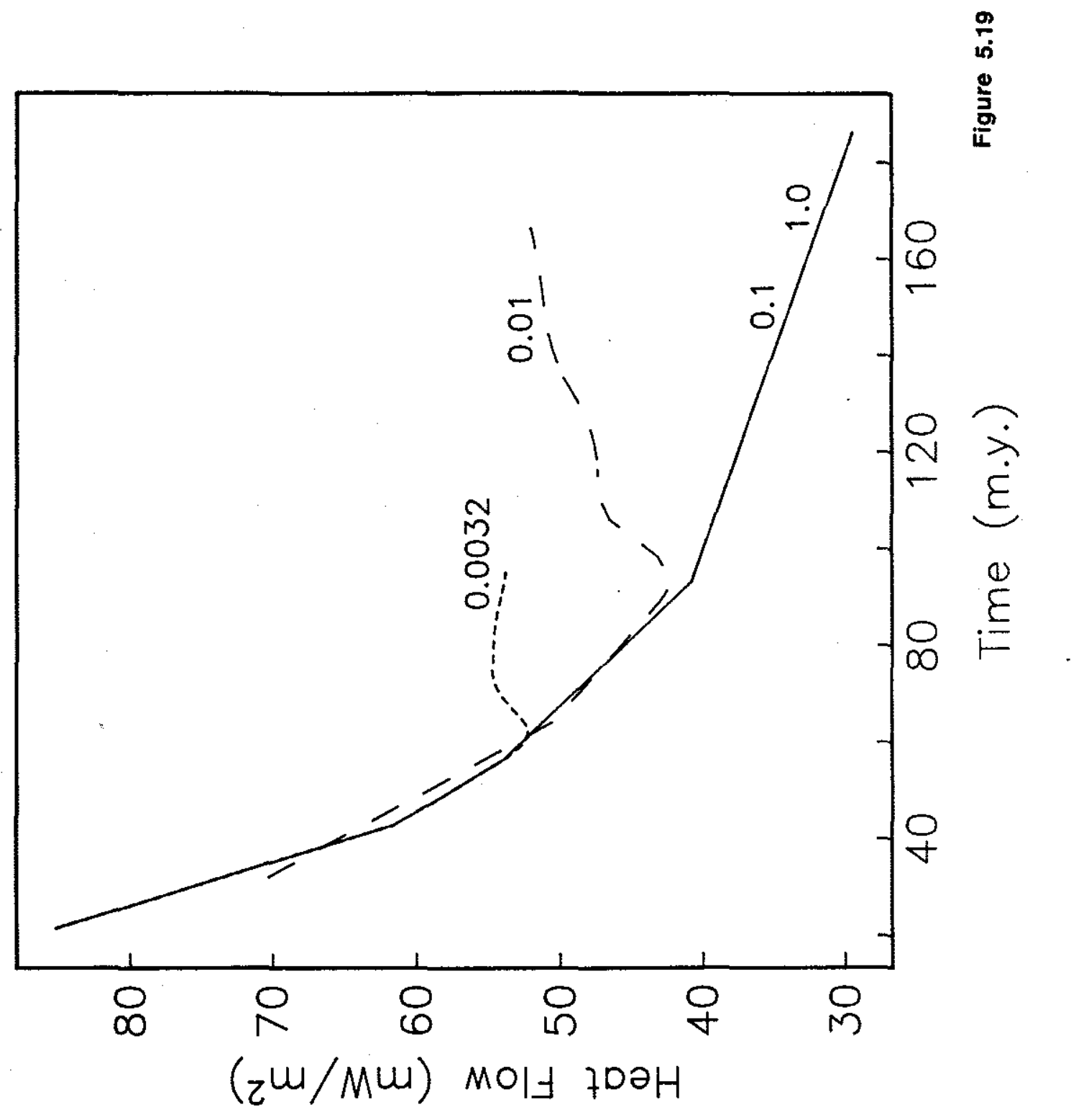


Chapter 6: CONCLUSIONS

We have explored the effect of a low viscosity zone in the uppermost mantle on mantle flow and its expression in gravity, geoid, topography and heat flow anomalies at the surface of the Earth. We examined three convective systems that are thought to exist in the mantle: (1) convection driven predominantly by heating from below (Chapters 2 and 3); (2) flow driven by the horizontal temperature gradient at a fracture zone (Chapter 4); and (3) instabilities under the cooling oceanic plates (Chapter 5). In each case, the same numerical model was employed, so that the results can be compared. In this chapter, we would like to take advantage of this similarity to draw some broad conclusions concerning the effect of a low viscosity zone on mantle convection and the oceanic plates.

\subsection{The Convective Flow}

In order to study the effect of a low viscosity zone on the flow, we simplified the finite element numerical model of the upper mantle to allow only three horizontal layers in the viscosity structure. The model consists of a conducting lid over a low viscosity zone which in turn is over a constant viscosity region extending to the base of the upper mantle. We can vary the box length, the thicknesses of the layers, the overall Rayleigh number, the method of heating, the boundary conditions and the viscosity contrast between the layers. As we vary these parameters, we explore the 
range of possible viscosity structures for the upper mantle. The numerical method is described in detail in the appendix. The viscosity enters the governing equations through the equation of motion. In particular, for a purely Newtonian fluid, the spatial derivatives of the velocity are scaled by the viscosity. Therefore, the velocities can change more rapidly inside the low viscosity zone than in the constant viscosity region, so that the top boundary layer encompasses higher velocities and the boundary layer is thinner. In the steady state convection problem driven by heating from below, this change in the velocity field causes the top boundary on the base of the conducting lid to appear, not rigid but, quasi-stress-free to the rest of the flow. Finally, in each of the problems, the transmission of normal stress to the conducting lid is diminished.

The response of the temperature field to changes in the velocity and stress fields depends on the temperature boundary conditions. In the steady state convection problem driven by a constant heat flux from below (Chapters 2 and $3)$, the temperature structure consists of cells with nearly constant temperatures in the interior encircled by thin, boundary layers. Inside the low viscosity zone, the boundary layers are thinner than those in the constant viscosity region (Figure 2.2). Since the heat flux is conserved in this model, the mean horizontal temperature gradient near the top boundary has the same magnitude in each case, but the isothermal region enlarges as we increase 
the viscosity contrast (Figure 2.2). Therefore, the mean temperature of the fluid decreases as the viscosity contrast increases.

In convective systems that are driven by a horizontal temperature gradient near the surface or driven by instabilities in a cooling boundary layer (Chapters 4 and $5)$, the low viscosity zone has the greatest influence on the first stages of the flow. If the viscosity contrast is high enough so that flow is confined to the low viscosity zone for a period of time, the convective flow is efficient at cooling the upper layer. The convection heats the uppermost parts of the low viscosity zone and cools the low portion of the layer. However, since the cooling is uneven (due to the initial temperature gradient in the fracture zone problem and due to quasi-random formation of instabilities in the small scale convection problem); a horizontal temperature gradient is created at the top of the lower layer. Convection then begins in the bottom layer and the flow eventually fills both layers. In fact, these results show that, due to the temperature gradients set up by the small scale convection atop a fluid with a supercritical Rayleigh number, flow will never be confined indefinitely to such a low viscosity zone. Once the temperature anomalies extend to the bottom boundary, the flow tends towards celis with an aspect ratio of one to one, and the boundary layers of these cells experience the same thinning as in the steady state convection problem. 
6.2 The Topographic, Geoid, Gravity and Heat Flow Response to Convective Temperature Anomalies at Depth.

The convective flow creates topography, geoid, gravity and heat flow anomalies at the surface through the exertion of normal stress onto the surface and through the presence of temperature anomalies. Since the low viscosity zone alters both the transmission of normal stress to the top boundary, it effects the response of the surface anomalies to the temperature anomalies at depth, as well as the convective flow. We calculate the response of the geoid, gravity and topography anomalies with a Green's function method which is explicitly discussed in Parsons and Daly (1983) (see Chapter 2 for the application of the method to these problems). With this method, since the viscosity structure is constant in the horizontal direction, the horizontal wavelengths decouple. We can then calculate the response of each wavelength in the anomalies to the temperature anomalies at depth.

We have drawn, in Figure 2.8, the appropriate response function, the "kernel", for the topography and gravity anomalies at a number of wavelengths for the viscosity structure defined in the figure caption. Because the low viscosity zone decreases the transmission of normal stress through the top layer, the topography kernel decreases with depth inside the low viscosity zone. In fact, at a viscosity contrast of two orders of magnitude and at wavelengths shorter than $600 \mathrm{~km}$, the topography kernel is almost zero below the low viscosity zone. Therefore, in the 
presence of a viscosity contrast, the temperature anomalies below this layer cannot effect the surface topography.

The kernels for the gravity and geoid anomalies are more complicated. The gravity and geoid fields reflect the sum of the gravitational effects of (1) the bottom boundary topography, (2) the top boundary topography and (3) the internal density distribution. The contributions of the latter two factors dominate the observables. Since the low viscosity zone blocks the effect on the surface topography of the temperature anomalies below the low viscosity zone and the topography decreases, the overall magnitude of the gravity anomalies also decreases. Moreover, because the effect of the internal density distribution does not change with the viscosity contrast, except through changes in the convective temperature distribution, the gravitational response of the internal density distribution to the temperature anomalies is larger than that of the surface topography at depths below the low viscosity zone at high viscosity contrasts. Since the response of the internal density distribution is opposite to that of the surface topography, the overall response of the gravity and geoid fields to the temperature anomalies below the low viscosity zone can be negative. This negative response counteracts the positive contributions to the temperature anomalies at shallower depths, so that the gravity and geoid anomalies decrease faster with viscosity contrast than the topography anomalies. 
The Green's function method to calculate the surface anomalies illustrates the importance of the viscosity structure in the response of the surface anomalies to temperatures at depth. Methods of calculating these anomalies which do not recognize these effects usually assume that the gravitational and topographic effects of the temperature anomalies below a certain depth, $d_{p}$, are negligible (McKenzie, 1967; Fleitout and Yuen, 1984b; Buck and Parmentier, 1986). Therefore, they are approximately correct for shorter wavelength features, near wavelengths comparable to $d_{p}$, but not for longer wavelengths. Furthermore, since the low viscosity zone diminishes the effect of temperature anomalies below its depth in the topography anomaly, but not in the gravity or geoid anomalies, these methods may predict topography (in part, by picking $d_{p}$ to be close to the base of the low viscosity zone at $200 \mathrm{~km}$ in depth), but radically err in predicting gravity and geoid.

\subsection{Constraints on the Viscosity structure of the Upper Mantle}

Applying the results of this model to the Earth's mantle involves a number of assumptions. First, the viscosity structure is horizontally averaged and held constant throughout the calculation. This simplification is required to reduce the computing time. However, it is also advantageous since the parameters of the calculation are more easy to control and specify, and the effects of the low 
viscosity zone are easier to identify. With the kernel method and a horizontally averaged viscosity structure, we can correctly calculate the topography and geoid anomalies for the convective system.

Second, our model is two-dimensional and advection in and out of the box is ignored. In particular, the shear flow due to the movement of the plates and to threedimensional coupling between the convective flows has been neglected. Some researchers are now exploring three dimensional models of the convective flow in the mantle and, perhaps soon, we will be able to quantify the effect of a third dimension in these convective systems.

Third, we have assumed that the convection is confined to the upper mantle. In each of the chapters, we discussed the effect of this bottom boundary on the convection and found that it had little effect on the surface observables, except in the fracture zone problem where the wavelength of the longest convective anomaly depends primarily on the depth of the fluid layer. Therefore, the results from these analyses can be applied to a whole mantle convection system with adjustments to the Rayleigh number and the scaling factors.

Finally, in order to compare the results of our model to data, we must use estimates for the scaling constants (Tables 2.1, 4.1 and 5.1). When the convection is predominantly near the surface, as in the fracture zone and small scale convection problems (Chapters 4 and 5), we chose 
values similar to the surface values. When the convection extends throughout the upper mantle, we used values appropriate for the upper mantle. However, since the kernels sample from all depths in the fluid, we have most likely erred in our selection of these constants. Ultimately other lines of evidence, such as those from experimental petrology, must be used to gauge accurately these constants.

With these caveats in mind, we can summarize the constraints that are placed on the viscosity structure of the uppermost mantle by the results of the studies in this thesis. The one observational constraint on the thickness of the low viscosity zone is the wavelength of the observed small scale convective anomalies in the Central Pacific (Chapter 5). Using the initial wavelength of $150-250 \mathrm{~km}$, the low viscosity zone must at first have a thickness of 75$125 \mathrm{~km}$. Theoretical predictions of the development of a low viscosity zone in the uppermost mantle indicate that the zone would thicken with age. In these calculations, to produce the observed depth-age relationships, the small scale convection and fracture zone models (Chapters 4 and 5) require a low viscosity zone at large ages. However, the low viscosity zone may thicken to $300-450 \mathrm{~km}$ as its effects are diminished.

The magnitude of the viscosity contrast is constrained in each of the studies. Both of the depth-age relationships predicted in the small scale convection and fracture zone 
problems (Chapters 4 and 5) require an average viscosity contrast at depth of at least two orders of magnitude until approximately $100 \mathrm{~m} \cdot \mathrm{y} .$. The existence of the long lived small scale anomalies in the SEASAT data set also requires a shallow low viscosity zone with a viscosity contrast of nearly two orders of magnitude in the uppermost mantle at ages up to $60 \mathrm{~m} . \mathrm{y}$. . Furthermore, if the average viscosity of the upper mantle were the value given by post-glacial rebound (1021 Pa.s; Peltier, 1974), then the size of the small scale anomalies and the absence of distinct convective anomalies in the fracture zone problem would require a viscosity contrast at depth of nearly two orders of magnitude. However, there is no independent evidence that the post-glacial rebound value of viscosity is correct and these observables, in particular, cannot distinguish between a overall lower viscosity for the upper mantle or a low viscosity zone.

The swell problem samples the viscosity structure of a small region. From our analyses of the Hawailan swell, the Bermudan swell, the Cape Verde Rise and the Marquesas swell, we have four sample "points" (Chapter 3). Although the interpretation of these results are complicated by the presence of hot temperatures associated with the plume which might decrease the overall viscosity underneath the plume, a distinct viscosity contrast was required at depth at each of the four swells that we studied. The magnitude of the viscosity contrast decreased with age from two orders of 
magnitude at the Marquesas swell (on $45 \mathrm{~m} . \mathrm{y}$. old Iithosphere) to one order of magnitude at the Cape verde Rise (on $130 \mathrm{~m} . \mathrm{y}$. Old lithosphere, McNutt, 1987). The magnitude of the viscosity contrasts required by the small scale convection, fracture zone and swell calculations and the decrease in magnitude with age as observed in the swell problem are consistent with theoretical temperature and pressure calculations of viscosity.

Since the viscosities in the top layer of the model contribute to the overall Rayleigh number of the fluid layers and significantly affect the shallow temperature anomalies which dominate the surface observables, we cannot accurately constrain the overall Rayleigh number of the upper mantle. In fact, with sufficient viscosity contrasts, Rayleigh numbers of $10^{5}$ to $10^{7}$ are consistent with our results (Chapters 3,4 and 5 ).

\subsection{Final Conclusions}

We therefore prefer an approximate model for the viscosity structure of the upper mantle which initially has a $125 \mathrm{~km}$ thick low viscosity zone that represents a viscosity contrast of two orders of magnitude. The viscosity contrast decreases as the plate ages to one order of magnitude or less by $130 \mathrm{~m} . \mathrm{y} .$, and the low viscosity zone may also thicken with age. Finally, the Rayleigh number of the upper mantle is at least $10^{5}$ and may be as large as $10^{7}$. With this model, the evolution of the surface plates would 
initially involve small scale convection which is driven by shear coupling to instabilities downstream and to small scale convection associated with fracture zones. This convective flow would begin at close to $5 \mathrm{~m} \cdot \mathrm{y}$. and remain confined to the low viscosity zone until nearly $40 \mathrm{~m} . \mathrm{y}$. . As this convective flow cools the upper mantle beneath the low viscosity zone, longer wavelength convection begins throughout the upper (or whole) mantle, and the heat transport from the longer wavelength convection flattens the depth-age curve and may influence the formation of swells. This model can then address and explain to some degree (1) the observed anomalies at mid-plate swells, (2) the evolution of the geoid and topographic steps at fracture zones, (3) the effect of the flow at fracture zones on the depth-age curve, (4) the small wavelength and coherent anomalies observed in the SEASAT data set in the Central Pacific, (5) the gravity and topographic anomalies observed aboard ship in the SEASAT anomaly region, (6) the flattening of the depth-age curve in the oceans, (7) the anomalously high heat flow values in comparison with the plate model observed at large ages in the Atlantic away from known thermal sources, and (8) the formation of those mid-plate swells which appear as the depth-age curve flattens. 
Appendix: THE NUMERICAL METHOD

To perform the convective flow calculations, we have used a velocity based finite element method. In this appendix, we describe here the formulation of the governing equations in finite element form, and give a rule of thumb which specifies the required grid size to resolve convective flows at a given Rayleigh number.

\section{A. 1 The Stokes Flow Formulation for Finite Elements}

The equation of motion describing stokes flow can be written using Einstein summation notation:

$$
\sigma_{i j, j}+f_{i}=0
$$

where $\sigma_{i j}$ is the deviatoric stress tensor and $f_{i}$ contains the body forces. In this thesis, we have restricted ourselves to the case of a Newtonian fluid for which the constitutive equation is given by:

$$
\sigma_{i j}=-p \delta_{i j}+\mu\left(u_{i, j}+u_{j, i}\right)
$$

where $p$ is the pressure, $\delta_{i j}$ is the Kronecker delta, $\mu$ is the dynamic viscosity and $u_{i}$ the velocity. The temperature structure is controlled by the heat equation:

$$
\partial \mathrm{T} / \partial t+u \cdot T_{i, j}=\kappa T_{i, j, i}
$$

where $\mathrm{K}$ is the thermal diffusivity. We shall also assume that the flow is incompressible.

$$
u_{i, i}=0
$$

A.2 The Penalty Function Formulation of the Pressure

In principle the finite element procedure can solve equations (A.1)-(A.4) with arbitrary boundary conditions. However, for numerical simplicity, we formulate the above 
equations using a penalty function representation for the pressure (Hughes et al.,1979). The constitutive equation can then be written:

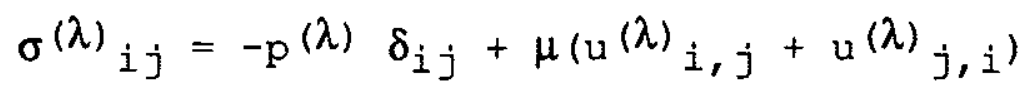

where

$$
\mathrm{p}^{(\lambda)}=-\lambda_{\mathrm{u}}(\lambda) \mathrm{k}, \mathrm{k}
$$

and where the magnitude of the parameter $\lambda(>0)$ is a machine dependent constant. The advantage of the penalty function method is that it eliminates the unknown variable $p$ and the necessity of independently satisfying the incompressibility condition. When the velocity is interpreted as a displacement vector, the equations of Stokes flow are the equations of isotropic, incompressible elasticity. With the penalty formulation, they are the equations of isotropic, compressible elasticity, and $\lambda$ and $\mu$ are the Lame parameters. Physically, in the fluids problem, the mass conservation equation is approximated so the associated errors represent net fluid loss or gain.

Temam (1977) has proved that the penalty function solution converges to the stokes flow solution. We outline his proof as follows. Subtract (2) from (5) to obtain

$$
\mu\left(u_{i}(\lambda)_{i}-u_{i}\right), j j-(p(\lambda)-p), i=0
$$

Multiplying each term by $\left(u(\lambda){ }_{i}-u_{i}\right)$, integrating over the fluid volume, $\Omega$ and employing the $H_{l}$ norm, given by:

$$
|u|=\left[\int \Omega(u u+u, i u, i) d \Omega\right]^{1 / 2}
$$

we obtain:

$$
\mu|u(\lambda)-u|^{2}+(1 / 2 \lambda)|p(\lambda)|^{2} \leqslant(1 / 2 \lambda)|p|^{2}
$$


Therefore, as $\lambda->, u(\lambda)->u$ and $p(\lambda)->p$. We shall use this method throughout the appendix, dropping the $\lambda$ superscripts. With use of the penalty method, some numerical points must be considered. The first concerns the size of $\lambda$, the penalty parameter. We need $\lambda$ large enough so that the compressibility is negligible, yet not so large as to produce numerical instability. Experimentation produces a condition on $\lambda$ as a function of the dynamic viscosity, $\mu$ (Hughes et al., 1979):

$$
\lambda=c \mu
$$

where $c$ depends on the word length characteristics of the computer. On Sun Microsystem workstations, on Apollo workstations and on a VAX780, in double precision, we set $\lambda$ equal to $10^{7}(\mu=1.0)$.

Another concern is the description of the pressure field. The pressure is constant in each element, but discontinuous between elements. Since bilinear elements cannot constrain pressure oscillations between elements, a "checkerboard" pattern can appear in the pressure field due to the element discontinuities. Despite these oscillations, the velocity field is accurate. (Hughes et al., 1979). However, to obtain a reasonable representation of the pressure field, we must filter out the checkerboard mode. Through a least squares smoothing procedure which redefines the pressure field in terms of the bilinear shape functions, we can both smooth the field and remove the checkerboard pattern to obtain a better picture of the pressure field. 


\section{A. 3 Matrix Formulation of the stokes Elow Problem}

We discretize our fluid volume, $\Omega$, into a number of elements, $\mathrm{n}_{e e}$, with volumes, $\Omega^{\mathrm{e}}$, and surfaces, $\Gamma^{\mathrm{e}}$. Associated with each of these elements are nodal points with indices: $I=\left(1,2, \ldots, n_{n p}\right)$. The position vector of the $i-$ th node is denoted $\underline{x}_{i}$ and the associated shape function is $S_{i}$

If we write the constitutive equation for each element as:

$$
\sigma^{e}=D^{e} B^{e} \underline{u}^{e}
$$

where $\mathrm{B}^{\mathrm{e}}$ is the local strain interpolation matrix, $\mathrm{D}_{e}$ is the local stress strain matrix and $\underline{u}^{e}$ stores the local nodal point coordinates, then the stiffness matrix $C$ will have two parts. The first corresponds to the pressure term in the Stokes equation:

$$
-p=D^{e} \lambda B^{e} \underline{U}^{e}
$$

and the second part to the viscous terms:

$$
\mu\left(u_{i, j}+u_{j, i}\right)=D^{e} B^{e} \underline{u}^{e}
$$

The components of the stiffness matrix can then be calculated using:

$c^{e}{ }_{a b}=\int \Omega^{e}\left(B^{e}\right)^{T} D^{e} \lambda B^{e}{ }_{b} d \Omega+\int \Omega^{e}\left(B^{e}{ }_{a}\right)^{T} D^{e}{ }_{\mu} B^{e} b_{b} d \Omega$ (A.14) For a two-dimensional finite element model, the stressstrain matrices are simply:

$$
D^{\mathrm{e}} \lambda=\lambda\left[\begin{array}{lll}
1 & 1 & 0 \\
1 & 1 & 0 \\
0 & 0 & 0
\end{array}\right] \quad ; \quad D^{\mathrm{e}} \mu=\mu\left[\begin{array}{lll}
2 & 0 & 0 \\
0 & 2 & 0 \\
0 & 0 & 1
\end{array}\right]
$$

and the strain interpolation matrix can be written: 


$$
B_{a}^{e}=\left[\begin{array}{cc}
s^{e} & 0 \\
0^{a}, 1 & s^{e^{a}, 2} \\
S^{e}, 2 & S^{e}, 1
\end{array}\right]
$$

The body forces, surface forces and concentrated forces can be summed into one vector, $\underline{\mathrm{R}}^{\mathrm{e}}$, given by:

$\underline{R}_{p}^{e}=\int \Omega^{e} S^{e} \underline{f}_{p} d \Omega+\int_{\Gamma^{e}} N^{e} \underline{h}_{p} d \Gamma-\sum_{q=1}^{n} C_{p q}^{e} q^{e} q \quad$ (A.17) where $\underline{f}_{p}$ is the body force, $\underline{h}_{p}$ is the surface force and $\underline{g}^{e} q$ is the concentrated load on node $q . n^{e} n p$ is the number of nodal points in the element $e$ and is equal to four for all of the bilinear (rectangular) elements. These matrices can be assembled to form a matrix equation in the global coordinate system:

$$
\mathrm{C} \underline{\mathrm{U}}=\underline{\mathrm{R}}
$$

Given the velocity field we can time step equation (A.3). Following the results of Brooks (1981) we use the streamline upwind Petrov-Galerkin method to treat the advective terms in the equation. Simple central difference methods produce solutions that do not exhibit enough diffusion. Upwind methods, on the other hand appear, overly diffuse. In one dimension, the upwind Petrov-Galerkin method optimizes a linear combination of the central difference method and upwind methods to produce the proper amount of diffusion and is nodally exact. To extend the method to two-dimensional flows, since the upwind effect is relevant only in the direction of flow, we apply it along streamlines. Using the above procedure for the advective term, the time stepping is performed using a predictorcorrector algorithm. 
A. 4 Resolution as a Function of Grid Size

When using finite difference methods, a rule of thumb is that, to resolve a convective flow, three nodes are needed across each convective boundary layer. We tried to formulate a similar rule of thumb for our finite element procedure. We took the case of a convective flow in a oneby-one box with a constant viscosity fluid interior, with rigid top and bottom boundaries, and with no-slip side boundaries. The flow is maintained by a heat flux through the bottom of the box; the temperature is held constant at the top of the box $(T=0)$; and reflective boundary conditions are imposed on the side boundaries.

With normalization, the heat flux should equal one throughout the box at steady state, so that no element is perpetually losing or gaining heat. Since the heat flux measures the accuracy at which the numerical solution is modelling the conductive and advective processes in the box, we will take as our convergence criterion a bound on the amount of variation in the heat flux with depth about the expected unit value. In finite difference calculations, the flow is considered to be resolved if the heat flux deviates by less than $1 \%$ (pers. comm. Steve Daly).

The heat flux, $q$, can be written as the sum of the conductive and the advective fluxes:

$$
q=-\partial T / \partial z+w T
$$

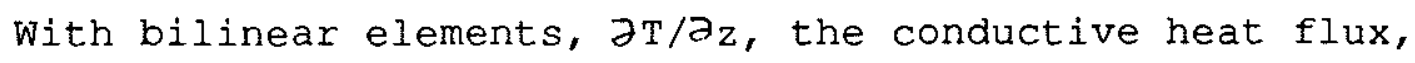
only varies in the horizontal direction throughout each 
element; however, WT is a quadratic term. Therefore, to accurately integrate the flux, one needs a $2 \times 2$ Gaussian integration rule. Using such an integration scheme, the heat flux was calculated for each element in a layer, then summed by layer and normalized by the volume of the layer.

As expected, the maximum errors in heat flux occur in the boundary layers. At a Rayleigh number of $10^{4}$, the boundary layers are not well defined but each encompasses about one fourth of the fluid depth. Therefore, a $16 \times 16$ grid has four elements in the boundary layer, an $8 \times 8$ grid has two elements in the layer and a $4 \times 4$ grid has one element. In this calculation, the $4 \times 4$ grid has a maximum of $8 \%$ error, the $8 \times 8$ grid has less than $2 \%$ error and the $16 \times 16$ grid has less than $0.8 \%$ error. At a Rayleigh number of $10^{5}$, the boundary layer is about $3 / 16$ of the layer depth so that a $16 \times 16$ grid has three elements in the layer, an $8 \times 8$ grid has less than two and a $4 \times 4$ grid has less than one. In this calculation, the $4 \times 4$ grid errs by a maximum of 128 , the $8 \times 8$ grid errs by less than 48 and the $16 \times 16$ grid errs by less than 18. In the middle of the box, in general, the errors are smaller. For example, they are below $0.1 \%$ for the $16 \times 16$ grid at a Rayleigh number of $10^{4}$. However, if we require less than 18 accuracy in the estimation of heat flow throughout the box then, at both Rayleigh numbers, only the $16 \times 16$ case resolves the flow.

Therefore, as with the finite difference techniques, close to three elements are required to span the boundary 
layer in order to obtain a resolved solution. In the calculations for this thesis, we followed this rule of thumb as an outer bound and three is the minimum number of elements that we used to resolve the boundary layer flow. 
REFERENCES

Anderson, D. L., and C. Sammis, Partial melting in the upper mantle, Phys. Earth Plan. Int., 3, 41, 1970.

Backus, G. E. and F. Gilbert, Numerical application of a formulism for geophysical inverse problems, Geophys. J. R. Astron. Soc., 13, 247, 1967.

Backus, G. E. and F. Gilbert, The resolving power of gross Earth data, Geophys. J. R. Astron. Soc., 16, 169, 1968.

Betz, F., and H. H. Hess, The floor of the Northern Pacific Ocean, Geog. Rev., 32, 99, 1942 .

Bonatti, E., and C. G. A. Harrison, Hot lines in the Earth's mantle, Nature, 263, 402, 1976.

Bott, M. H. P., The Interior of the Earth: Its structure Constitution and Evolution, Elsevier Science Publishing Co., New York, New York, 1982.

Brooks, A. N., A Petrov-Galerkin Einite Element Formulation for Convection Dominated Flows, Ph.D. Thesis, Cal. Inst. of Tech., 1981.

Buck, W. R., and E. M. Parmentier, Convection beneath young oceanic lithosphere: Implications for thermal structure and gravity, J. Geophys. Res., 91, 1961, 1986.

Busse, F. H., The stability of finite amplitude cellular convection and its relation to an extremum principle, I. Fluid Mech., 30, 625, 1967.

Busse, F. H. and J. A. Whitehead, Instabilities of convection rolls in a high Prandtl number fluid, J. Fluid Mech., 47, 305, 1971.

Busse, F. H. and J. A. Whitehead, Oscillatory and collective instabilities in a large Prandtl number convection, $\mathrm{J}$. Eluid Mech., 66, 67, 1974.

Cazenave, A., Thermal cooling of the lithosphere: New constraints from geoid height data, Earth and Planet. Sci. Lett., 70, 395, 1984.

Cazenave, A., Lago, B., and Dominh, K., Geoid anomalies over the northeast Pacific fracture zones from satellite altimeter data, Geophys. J.R. Astron. Soc., 69, 15, 1982 . 
Cazenave, A., Monnereau, M., and Gibert, D., SEASAT gravity undulations in the Central Indian ocean, submitted to J. Geophys. Res., 1987.

Cooper, R. F. and D. L. Kohlstedt, Solution-precipitation enhanced diffusional creep of partially molten olivine basalt aggregates during hot-pressing, Tectonophysics, $107,207,1984$.

Courtney, R. C., and R. S. White, Anomalous heat flow across the Cape Verde Rise: Evidence for a thermal plume in the Earth's mantle, Geophys. J.R. Astron. Soc., 87, $815,1986$.

Craig, C. H., and D. P. McKenzie, The existence of a thin low viscosity layer beneath the lithosphere, Earth and Plan. Sci. Lett., 78, 420, 1986.

Crough, S. T., The thermal origin of mid-plate hotspot swells, Geophys. J.R. Astron. Soc., 55, 451, 1978 .

Crough, S. T., Hotspot epeirogeny, Tectonophysics, 61,321 , $1979 a$.

Crough, S. T., Geoid anomalies across fracture zones and the thickness of the lithosphere, Earth and Planet. Sci. Lett., 44, 224, $1979 \mathrm{~b}$.

Crough, S. T., and R. D. Jarrard, The Marquesas-Line swell, J. Geophys. Res., 86, 11763, 1981.

Daly, S.F., B. Parsons and A. Raefsky, Layered convection and its effect on gravity anomalies and surface topography, EOS, Trans. Amer. Geophys. Un., 65, 1092, 1984 .

Daly, S. F., and A. Raefsky, on the penetration of a hot diapir through a strongly temperature-dependent viscosity medium, Geophys. J. R. Astron. Soc., 83, 657, 1985 .

Dash, B. P., M. M. Bell, G. A. King, L. W. Butler, and P: M. Rona, Geophysical investigation of the Cape Verde Archipelago, J. Geophys. Res., 81, 5249, 1976.

Davis, E. E., Lister C. R. B. and Sclater, J. G., 1984. Towards determining the thermal state of old ocean lithosphere: heat flow measurements from the BlakeBahama outer ridge, N. W. Atlantic, Geophys. J. R. Astron. Soc., 78, 507 . 
Detrick, R. S., An analysis of geoid anomalies across the Mendocino fracture zone: implications for thermal models of the lithosphere, J. Geophys. Res., 87, 11751, 1981 .

Detrick, R. S., and S. T. Crough, Island subsidence, hot spots, and lithospheric thinning, J. Geophys. Res., 83, 1236,1978 .

Detrick, R. S., R. P. von Herzen, S. T. Crough, D. Epp, and U. Fehn, Heat flow on the Hawaiian swell and

lithospheric reheating, Nature, 292, 142, 1981.

Detrick, R. S., R. P. von Herzen, B. Parsons, D. Sandwell, and $M$. Dougherty, Heat flow observations on the Bermuda rise and thermal models of mid-plate swells, J. Geophys. Res., 91, 3701, 1986.

Dietz, R. S., and H. W. Menard, Hawaiian swell, deep, and arch, and subsidence of the Hawaiian islands, Jour. Geology, 61, 99, 1953.

Driscoll, M. L., Eischer, R. L., and Parsons, B., Fracture zone trends and structure at the Southwest Indian Ridge: An investigation using seasat altimetry and surface-ship bathymetry, Geophys. J. R. Astron. Soc., in press, 1987 .

Driscoll, M. L. and Parsons, B., Cooling of the oceanic lithosphere: evidence from geoid anomalies across the Udintsev and Eltanin fracture zones, Earth Planet. Sci. Lett., sub judice, 1987.

Emerman, S. H., and D. L. Turcotte, stagnation flow with a temperature dependent viscosity, J. Fluid Mech., 127, $507,1983$.

Fischer, K. M., M. K. McNutt and L. Shure, Thermal and mechanical constraints on the lithosphere beneath the Marquesas swell, Nature, $322,733,1986$.

Fleitout, L., and Yuen, D. A., Secondary convection and the growth of the oceanic lithosphere, phys. Earth planet. Inter., 36, 181, $1984 \mathrm{a}$.

Fleitout, L., and D. A. Yuen, Steady state, secondary convection beneath lithospheric plates with temperature- and pressure-dependent viscosity, $\mathrm{J}$. Geophys. Res., 89, 9227, $1984 \mathrm{~b}$.

Eorsyth, D. W., The evolution of the upper mantle beneath mid-ocean ridges, Tectonophysics, 38, 89, 1977. 
Forsyth, D. W. and S. Uyeda, on the relative importance of the driving force of plate motion, Geophys. J.R. Astron. Soc., 43, 163, 1975.

Foster, T. D., Stability of a homogeneous fluid cooled uniformly from above, phys. of Fluids, $\underline{8}, 1249,1965 \mathrm{a}$.

Foster, T. D., Onset of convection in a layer of fluid cooled from above, Phys. of Fluids, $\underline{8}, 1770,1965 \mathrm{~b}$.

Freedman, A. and Parsons, B., Geoid anomalies over two south Atlantic fracture zones, sub judice, 1987.

Haxby, W. F., and D. L. Turcotte, on isostatic geoid anomalies, J. Geophys. Res., 83, 5473, 1978.

Haxby, W. F., and J. K. Weissel, Evidence for small scale mantle convection from SEASAT altimeter data, J. Geophys. Res., 91, 3507, 1986.

Heestand, R. L., and Crough, S. T., The effect of hotspots on the age-depth relation, J. Geophys. Res., 86, 1981.

Houseman, G., and D. P. McKenzie, Numerical experiments on the onset of convective instability in the Earth's mantle, Geophys. J. R. Astron. Soc., 79, 2961, 1982.

Hughes, T.J.R., W.K. Liu and A. Brooks, Review of finite element analysis of incompressible viscous flows by the penalty function formulation, J. Computational Phys., 30, 1-60, 1979 .

Jarrard, R. D., and D. A. Clague, Implications of Pacific island and seamount ages for the origin of volcanic chains, Rev. Geophys. and Space Phys., 15, 57, 1977.

Jarvis, G. T., and Peltier, W. R., Oceanic bathymetry profiles flattened by radiogenic heating in a convecting mantle, Nature, 285, 649, 1980.

Jarvis, G. T., and Peltier, W. R., Mantle convection as a boundary layer phenomenon, Geophys. J. R. astron. Soc., $\underline{68}, 385,1982$.

Jaupart, C. and B. Parsons, Convective instabilities in a variable viscosity fluid cooled from above, Phys. of Earth Plan. Int., 39, 14, 1985.

Knill, R. A., Isotopic evidence for age of uplift of the Cape Verde Rise (abstr.), Geophys. J.R. Astron. Soc., $\underline{81}, 344,1985$. 
Knopoff, L., Thermal convection in the Earth's mantle, in The Earth's Mantle, ed. T. F. Gaskel1, 171, Academic, New York, 1967.

Lancelot, Y., E. Seibold, et al., Initial Reports of the Deep Sea Drilling Project, vol. 41 , washington D.C., 1977 .

Langseth, M. G., LePichon, X., and Ewing, M., Crustal structure of mid-ocean ridges, 5, Heat flow through the Atlantic ocean floor and convection currents, J. Geophys. Res., 71, 5321, 1966.

Louden, K. E., Wallace, D. O., and Courtney, R. C., Heat flow and depth versus age for the Mesozoic NW Atlantic ocean: Results from the Sohm abyssal plain and implications for the Bermuda Rise, Earth and Planet. Sci. Lett., in press, 1987.

Mckenzie, D. P., Some remarks on heat flow and gravity anomalies, J. Geophys. Res., 72, 6261, 1967.

McKenzie, D. P., Speculations on the causes and consequences of plate motions, Geophys. J.R. Astron. Soc., 18, 1, 1969.

McKenzie, D. P., The Earth's mantle, Sci. Amer., 249, 66, 1983 .

Mckenzie, D. P., The generation and compaction of partially molten rock, J. Petrol., 25, 713, 1984.

McKenzie, D.P., The extraction of magma from the crust and mantle, Earth Plan. Sci. Lett., 74, 81-91, 1985.

Mckenzie, D. P., and Richter, F., Parameterized thermal convection in a layered region and the thermal history of the Earth, J. Geophys. Res., 86, 11667, 1981.

McKenzie, D. P., J. M. Roberts, and N. O. Weiss, Convection in the Earth's mantle: Towards a numerical simulation, J. Eluid Mech., 62, 465, 1974.

McKenzie, D. P., A. Watts, B. Parsons, and M. Roufosse, Planform of mantle convection beneath the Pacific ocean, Nature, $288,442,1980$.

McNutt, M.K., Lithospheric flexure and thermal anomalies, J. Geophys. Res., 89, 11,180-11,194, 1984 .

McNutt, M. K., Thermal and mechanical properties of the Cape Verde rise, J. Geophys. Res., in press, 1987. 
McNutt, M. K., and K. M. Fischer, The South Pacific superswell, Seamounts, Islands and Atolls, B. Keating and R. Batiza, eds, Geomona series, AGU, Washington D.C., 1987 .

McNutt, M. K., and H. W. Menard, Constraints on yield strength in the observations of flexure, Geophys. J. R. Astron. Soc., 71, 363, 1982 .

McNutt, M. K., and L. Shure, Estimating the compensation depth of the Hawaiian swell with linear filters, J. Geophys. Res., 91, 13,915, 1986.

Menard, H. W., and M. K. McNutt, Evidence for and consequences of thermal rejuvenation, J. Geophys. Res., $87,8570,1982$.

Molnar, P., and T. Atwater, Relative motion of hotspots in the mantle, Nature, 246, 288, 1973.

Parker, R. I., Understanding inverse theory, Ann. Rev. Earth Planet. Sci., 5, 35, 1977.

Parker, R. L., and Oldenburg, D. W., Thermal model of ocean ridges, Nature, 242, 137, 1973.

Parmentier, E. M., A study of thermal convection in nonNewtonian fluids, J. Fluid Mech., 84, 1, 1978.

Parmentier, E. M., and Haxby, W. F., 1986. Thermal stresses in the oceanic lithosphere: Evidence from geoid anomalies at fracture zones, J. Geophys. Res., 91, 7193.

Parmentier, E. M., and Turcotte, D. L., Two dimensional mantle flow beneath a rigidly accreting lithosphere, Phys. Earth Planet. Inter., 17, 281, 1978.

Parsons, B., and S. F. Daly, The relationship between surface topography, gravity anomalies and temperature structure of convection, J. Geophys. Res., 88, 1129, 1983.

Parsons, B. and S.F. Daly, Convection beneath a conducting lid: Implications for the origins of mid-plate swells, EOS, Trans. Amer. Geophys. Un., 65, 1087, 1984.

Parsons, B., and D. P. McKenzie, Mantle convection and the thermal structure of the plates, J. Geophys. Res., 83, 4485, 1978 .

Parsons, B., and Richter, F., A relation between the driving force and the geoid anomaly associated with mid-ocean ridges, Earth and Planet. Sci. Lett., 51, 445, 1980. 
Parsons, B., and J. G. Sclater, An analysis of the variation of the ocean floor bathymetry and heat flow with age, J. Geophys. Res., $\underline{82}, 803,1977$.

Peltier, W. R., The impulse response of a Maxwell Earth, Rev. Geophys. Space Phys., 12, 649, 1974.

Peltier, w. R., Glacial isostatic adjustment - II the inverse problem, Geophys. J. R. astron. Soc., 46, 669, 1976.

Peltier, W. R., Ice age dynamics, Annu. Rev. Earth Planet. Sci., $9,199,1981$.

Rayleigh, Lord, on convection currents in a horizontal layer of fluid when the high temperature in on the under side, Phi1. Mag., Ser. 6, 32, 529, 1916.

Richter, F. M., Convection and the large scale circulation of the mantle, J. Geophys. Res., 78, 8735, 1973.

Richter, F. M. and D. P. McKenzie, Simple plate models of mantle convection, J. Geophys. Res., 44, 441, 1978.

Richter, F. M., and Parsons, B., On the interaction of two scales of convection in the mantle, J. Geophys. Res., $\underline{80}, 2529,1975$.

Roberts, P. H., Convection in horizontal layers with internal heat generation, Theory. J. Fluid Mech., 30, 33,1967 .

Robinson, E. M., and Parsons, B., The effect of a shallow low viscosity zone on the formation of mid-plate swells, submitted to J.Geophys. Res., 1987 a.

Robinson, E. M., and B. Parsons, The effect of a shallow low viscosity zone on small scale instabilities under the cooling oceanic plates, submitted to J. Geophys. Res., $1987 \mathrm{~b}$.

Robinson, E. M., B. Parsons and S. F. Daly, The effect of a low viscosity zone on the apparent compensation of midplate swells, Earth and Plan. Sci. Lett., 82, 335, 1987 a.

Robinson, E. M., B. Parsons and M. Driscoll, The effect of a low viscosity zone on the mantle flow and geoid anomalies at fracture zones, Geophys. J.R. astron. Soc., in press, $1987 \mathrm{~b}$. 
Runcorn, S. K., Convection in the mantle, in The Earth's Crust and Upper Mantle, Geophys. Monogr. Ser., 13, ed. P. J. Hart, p. 692, AGU, Washington, D. C., 1969 .

Sandwell, D. T., Thermomechanical evolution of oceanic fracture zones, J.Geophys. Res., 89, 11401, 1984.

Sandwell, D., and Schubert, G., Geoid height-age relation from seasat altimeter profiles across the Mendocino fracture zone, J. Geophys. Res., 87, 3949, 1982.

Schubert, G., Frodeveaux, C., and Yuen, D. A., Oceanic lithosphere and asthenosphere: thermal and mechanical structure, J. Geophys. Res., 77, 945, 1976.

Sclater, J. G., Anderson, R. N., and Bell, M. L., Elevation of ridges and the evolution of the central eastern Pacific, J. Geophys. Res., 76, 7888, 1971.

Sclater, J. G., and Francheteau, J., The implications of terrestrial heat flow observations on current tectonic and geochemical models of the crust and upper mantle of the Earth, Geophys. J. R. Astron. Soc., 20, 509, 1970.

Sclater, J. G., and L. Wixom, The relationship between depth and age and heat flow and age in the Western North At lantic, in The Geology of North America: The Western Atlantic Region, DNAG series, edited by $B$. Tucholke and P. R. Vogt, Geol. Soc. of America, Boulder, 1986.

Solomon, S. C., Seismic wave attenuation and partial melting in the upper mantle of North America, J. Geophys. Res., 77, 1483, 1972 .

Stillman, C. J., H. Furnes, M. J. Le Bas, A. H. F. Robertson, and J. Zielonka, The geological history of Maio, Cape Verde Islands, J. Geol. Soc., 139, 347, 1982 .

Talwani, M., Gravity, in The Sea, 4, pt. 1, ed. A. E. Maxwel1, McGraw-Hill, 251, 1958.

Tapley, B. D., G. H. Born and M. E. Park, The SEASAT altimeter data and its accuracy assessment, J.Geophys. Res., 87, 3179, 1982 .

Temam, R., Navier-Stokes Equations, North-Holland, 1977.

Turcotte, D. L., and E. R. Oxburgh, Finite amplitude convective cells and continental drift, J. Fluid Mech., $\underline{28}, 29,1967$. 
Von Herzen, R. P., R. S. Detrick, S. T. Crough, D. Epp, and U. Fehn, Thermal origin of the Hawaiian swell: heat flow evidence and thermal models, J. Geophys. Res., 87, 6711, 1982.

Watts, A., Gravity and bathymetry in the Central Pacific Ocean, J. Geophys. Res., 81, 1533, 1986.

Watts, A., An analysis of isostasy in the world's oceans: 1: Hawaiian-Emperor seamount chain, J. Geophys. Res., 83, 5989, 1978 .

Weilandt, E., and L. Knopoff, Dispersion of very long period Rayleigh waves along the East Pacific Rise: Evidence for $S$ wave velocity anomalies down to $450 \mathrm{~km}$ depth, I. Geophys. Res., 87, 8631, 1982.

Young, R., and I. A. Hill, An estimate of the effective elastic thickness of the Cape Verde rise, J. Geophys. Res., 91, 4854, 1986.

Yuen, D. A., and L. Fleitout, Thinning of the lithosphere by smal1-scale convective destabilization, Nature, 313, 125,1985 .

Yuen, D. A., Peltier, W. R., and Schubert, G., On the existence of a second scale of convection in the upper mantle, Geophys. J. R. Astron. Soc., 65, 171, 1981. 


\section{LIST OF RUNS}

SWELI PROBLEM (Chapters 2 and 3):

\begin{tabular}{|c|c|c|c|c|c|c|}
\hline $\begin{array}{l}1 . \\
2 . \\
3 . \\
4 .\end{array}$ & $\begin{array}{l}\text { LID } \\
\text { THICK. } \\
0.125 \\
0.125 \\
0.125 \\
0.125\end{array}$ & $\begin{array}{l}\text { LVL } \\
\text { THICK. } \\
0.21 \\
0.21 \\
0.21 \\
0.21\end{array}$ & $\begin{array}{l}\text { RAYLEIGH } \\
\text { NUMBER } \\
1.0 \times 10^{4} \\
1.0 \times 10^{4} \\
1.0 \times 10^{4} \\
1.0 \times 10^{4}\end{array}$ & $\begin{array}{l}\mu_{\mathrm{t}} / \mu_{\mathrm{b}} \\
1.0 \\
0.1 \\
0.01 \\
0.001\end{array}$ & $\begin{array}{l}\text { GRID } \\
\text { SIZE } \\
24 \times 27 \\
24 \times 32 \\
24 \times 42 \\
24 \times 42\end{array}$ & $\begin{array}{l}\text { FIGURE } \\
\text { NUMBER }\end{array}$ \\
\hline $\begin{array}{l}5 . \\
6 . \\
7 .\end{array}$ & $\begin{array}{l}0.125 \\
0.125 \\
0.125\end{array}$ & $\begin{array}{l}0.21 \\
0.21 \\
0.21\end{array}$ & $\begin{array}{l}3.2 \times 10^{4} \\
3.2 \times 10^{4} \\
3.2 \times 10^{4}\end{array}$ & $\begin{array}{l}1.0 \\
0.1 \\
0.01\end{array}$ & $\begin{array}{l}24 \times 27 \\
24 \times 32 \\
24 \times 42\end{array}$ & \\
\hline $\begin{array}{l}8 . \\
9 . \\
10 . \\
11 . \\
12 . \\
13 . \\
14 .\end{array}$ & $\begin{array}{l}0.125 \\
0.125 \\
0.125 \\
0.125 \\
0.125 \\
0.125 \\
0.125\end{array}$ & $\begin{array}{l}0.21 \\
0.21 \\
0.21 \\
0.21 \\
0.21 \\
0.21 \\
0.21\end{array}$ & $\begin{array}{l}1.0 \times 10^{5} \\
1.0 \times 10^{5} \\
1.0 \times 10^{5} \\
1.0 \times 10^{5} \\
1.0 \times 10^{5} \\
1.0 \times 10^{5} \\
1.0 \times 10^{5}\end{array}$ & $\begin{array}{l}1.0 \\
0.1 \\
0.075 \\
0.050 \\
0.025 \\
0.01 \\
0.001\end{array}$ & $\begin{array}{l}24 \times 27 \\
24 \times 42 \# \\
24 \times 42 \\
24 \times 42 \\
24 \times 42 \\
24 \times 42 \# \\
24 \times 42 \#\end{array}$ & $\begin{array}{l}2.2,3.2 \\
3.2\end{array}$ \\
\hline $\begin{array}{l}15 . \\
16 . \\
17 .\end{array}$ & $\begin{array}{l}0.125 \\
0.125 \\
0.125\end{array}$ & $\begin{array}{l}0.21 \\
0.21 \\
0.21\end{array}$ & $\begin{array}{l}3.2 \times 10^{5} \\
3.2 \times 10^{5} \\
3.2 \times 10^{5}\end{array}$ & $\begin{array}{l}1.0 \\
0.1 \\
0.01\end{array}$ & $\begin{array}{l}24 \times 27 \\
24 \times 42 \\
24 \times 42\end{array}$ & \\
\hline $\begin{array}{l}18 . \\
19 . \\
20 .\end{array}$ & $\begin{array}{l}0.125 \\
0.125 \\
0.125\end{array}$ & $\begin{array}{l}0.21 \\
0.21 \\
0.21\end{array}$ & $\begin{array}{l}1.0 \times 10^{6} \\
1.0 \times 10^{6} \\
1.0 \times 10^{6}\end{array}$ & $\begin{array}{l}1.0 \\
0.1 \\
0.01\end{array}$ & $\begin{array}{l}24 \times 27 \\
24 \times 42 \\
24 \times 42\end{array}$ & $2.4,3.3$ \\
\hline $\begin{array}{l}21 . \\
22 . \\
23 . \\
24 .\end{array}$ & $\begin{array}{l}0.125 \\
0.125 \\
0.125 \\
0.125\end{array}$ & $\begin{array}{l}0.083 \\
0.083 \\
0.083 \\
0.083\end{array}$ & $\begin{array}{l}1.0 \times 10^{4} \\
1.0 \times 10^{5} \\
1.0 \times 10^{5} \\
1.0 \times 10^{5}\end{array}$ & $\begin{array}{l}0.1 \\
0.1 \\
0.01 \\
0.001\end{array}$ & $\begin{array}{l}24 \times 29 \\
24 \times 29 \# \\
24 \times 29 \# \\
24 \times 29\end{array}$ & $2.6,3.7$ \\
\hline $\begin{array}{l}25 . \\
26 . \\
27\end{array}$ & $\begin{array}{l}0.125 \\
0.125 \\
0.125\end{array}$ & $\begin{array}{l}0.5 \\
0.5 \\
0.5\end{array}$ & $\begin{array}{l}1.0 \times 10^{4} \\
1.0 \times 10^{5} \\
1.0 \times 10^{5}\end{array}$ & $\begin{array}{l}0.1 \\
0.1 \\
0.01\end{array}$ & $\begin{array}{l}24 \times 40 \\
24 \times 40 \\
24 \times 40\end{array}$ & $2.6,3.7$ \\
\hline $\begin{array}{l}28 . \\
29 .\end{array}$ & $\begin{array}{l}0.125 \\
0.125\end{array}$ & $\begin{array}{l}0.75 \\
0.75\end{array}$ & $\begin{array}{l}1.0 \times 10^{4} \\
1.0 \times 10^{5}\end{array}$ & $\begin{array}{l}0.1 \\
0.1\end{array}$ & $\begin{array}{l}24 \times 46 \\
24 \times 46\end{array}$ & \\
\hline $\begin{array}{l}30 . \\
31 . \\
32 .\end{array}$ & $\begin{array}{l}0.0 \\
0.042 \\
0.083\end{array}$ & $\begin{array}{l}0.0 \\
0.0 \\
0.0\end{array}$ & $\begin{array}{l}1.0 \times 10^{5} \\
1.0 \times 10^{5} \\
1.0 \times 10^{5}\end{array}$ & $\begin{array}{l}1.0 \\
1.0 \\
1.0\end{array}$ & $\begin{array}{l}24 \times 24 \\
24 \times 25 \\
24 \times 26\end{array}$ & \\
\hline
\end{tabular}


FRACTURE ZONE PROBLEM (Chapter 4):

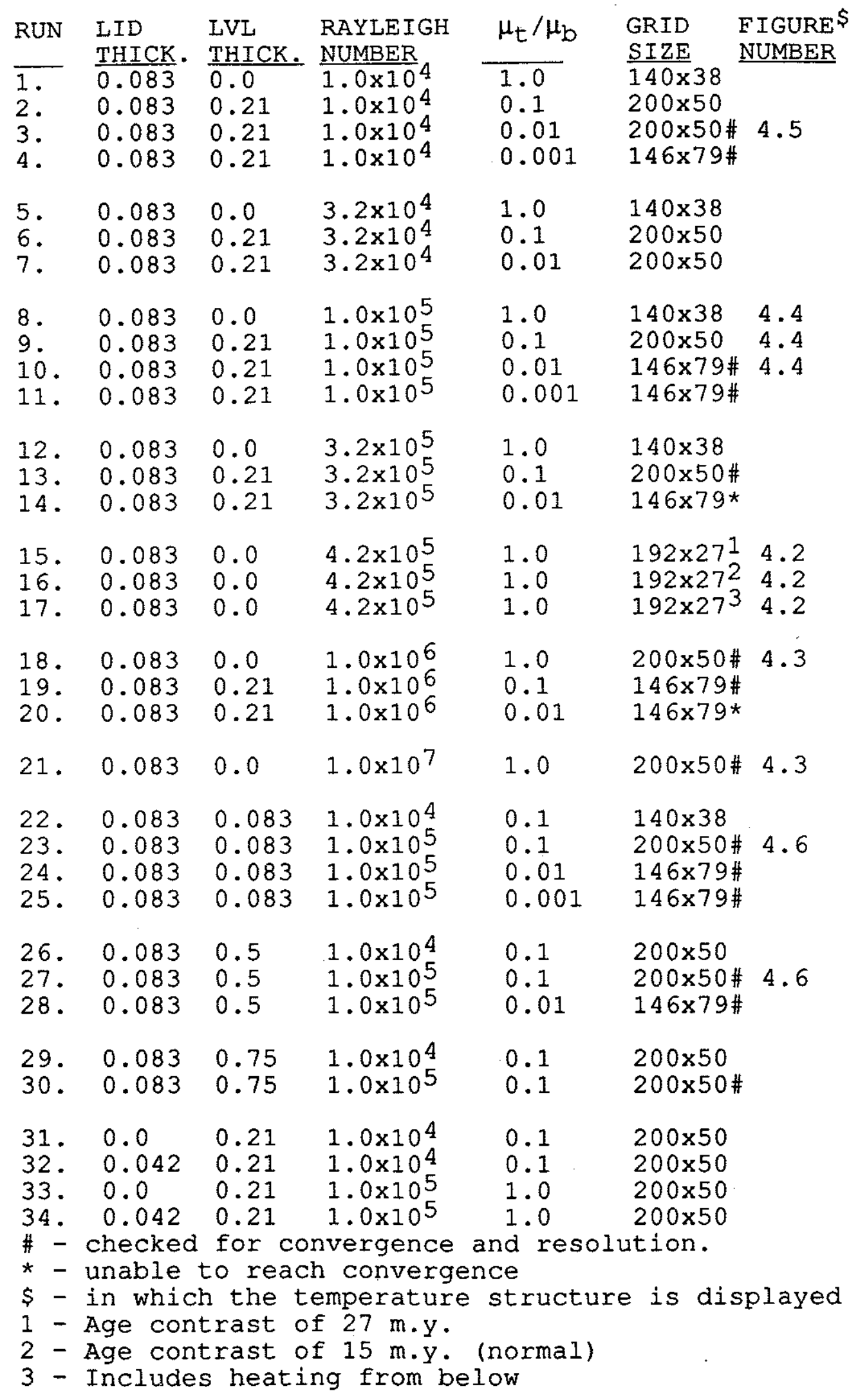


COOLING INSTABILITIES PROBLEM (Chapter 5):

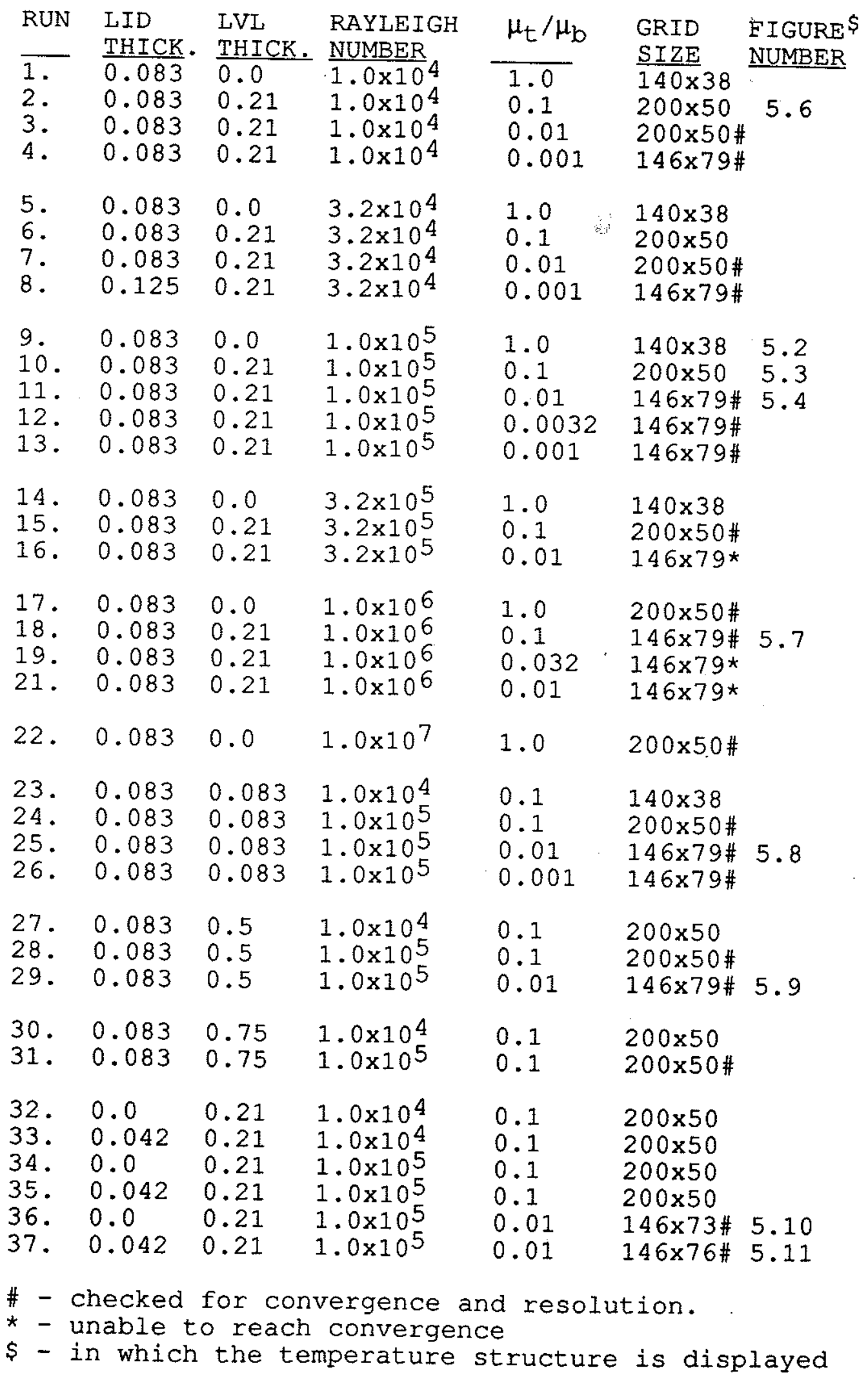

